\title{
ACMT 2020 Annual Scientific Meeting Abstracts - New York, NY
}

\begin{abstract}
These are the abstracts of the 2020 American College of Medical Toxicology (ACMT) Annual Scientific Meeting. Included here are 174 abstracts that will be presented in March 2020, including research studies from around the globe and the ToxIC collaboration, clinically significant case reports describing new toxicologic phenomena, and encore research presentations from other scientific meetings.
\end{abstract}

Keywords Abstracts, Annual Scientific Meeting, Toxicology Investigators Consortium, Medical Toxicology Foundation

Correspondence: American College of Medical Toxicology (ACMT), 10645 N. Tatum Blvd, Phoenix, AZ, USA; info@acmt.net

Introduction: The American College of Medical Toxicology (ACMT) received 223 eligible research abstracts for consideration for presentation at the 2020 Annual Scientific Meeting (ASM), including 107 research studies and 116 case reports. Each abstract was reviewed in a blinded fashion by at least four research committee members. Each abstract was independently scored based on the clinical question, data source, analytic method, results/conclusion, and clarity of presentation. A total of 177 abstracts were accepted. This work would not be possible without the hard work and diligence of our abstract reviewers: Justin Arnold, Katie Boyle, Jeffrey Brent, Keith Burkhart, Diane Calello, Stephanie Carreiro, James Chenoweth, Neeraj Chhabra, Nick Connors, Kirk Cumpston, Rob Hendrickson, Michelle Hieger, David Jang, David Juurlink, Louise Kao, Kenneth Katz, Kate Katzung, Russ Kerns, Andrew King, Kathy Kopec, JoAn Laes, Eric Lavonas, Michael Levine, David Liss, Gerald Maloney, Kevin Maskell, William Meggs, Andrew Monte, Elissa Moore, Mark Mycyk, Travis Olives, Patricia Rosen, Evan Schwarz, Daniel Sessions, Samuel Stellpflug, Mark Su, Manoj Tyagi, Shawn Varne, Steven Walsh, Richard Wang, Sage Wiener, Brandon Wills, and Luke Yip. Equally significant is the contribution of the ACMT staff (Adrienne Dunavin, Dana Karshenas, and Lizzy Nguyen) who led the process. Congratulations to all the contributors whose work will be presented in New York.

We look forward to seeing you there.

Jon B. Cole, MD, FACMT, Abstract Review Chair; Christine Murphy, MD, FACMT, Abstract Review Co-Chair; Maryann Amirshahi, PharmD, MD, MPH, PhD, FACMT, Chair, ACMT Research Committee

\section{ACMT 2020 Annual Scientific Meeting Abstracts - New York, NY}

\section{Day 1: Platforms, Abstracts 001-004}

001. Efficacy of Oral Sodium Thiosulfate for Acute, Severe Oral Cyanide Toxicity in Swine

Patrick C Ng${ }^{1}$, Tara B Hendry-Hofer ${ }^{2}$, Joseph K Maddry ${ }^{3}$, Vikhyat S Bebarta $^{2}$

${ }^{1}$ Brooke Army Medical Center, Fort Sam Houston, TX, USA. ${ }^{2}$ University of Colorado Anschutz Medical Campus, Aurora, CO, USA. ${ }^{3}$ En Route Care and Research Center, Fort Sam Houston, TX, USA
Background: Oral cyanide is a potentially deadly poison and has the potential for use by terrorists. There is potential for a mass casualty exposure scenario, and currently, there are no FDA-approved antidotes specifically for oral cyanide poisoning.

Hypothesis: We hypothesize that animals treated with oral sodium thiosulfate will have a higher rate of survival vs. control in a large animal model of acute, severe, oral cyanide toxicity.

Methods: This is a prospective study that took place at the University of Colorado Anschutz Medical Campus. Nine swine (45-55 kg) were instrumented, sedated, and stabilized. Potassium cyanide $(8 \mathrm{mg} / \mathrm{kg} \mathrm{KCN})$ in saline was delivered as a one-time bolus via an orogastric tube. Three minutes after cyanide, animals who were randomized to the treatment group received sodium thiosulfate $(508.2 \mathrm{mg} / \mathrm{kg}, 3.25 \mathrm{M}$ solution) via orogastric tube. Survival at 60 minutes after exposure was the primary outcome. We compared survival between treatment and control groups by log-rank, Mantel-Cox analysis, and trended labs and vital signs.

Results: At baseline and time of treatment, all animals were similar. Survival at 60 minutes was $100 \%$ in treated animals compared to $0 \%$ in the control group $(p=0.0027)$. Animals in the control group became apneic and subsequently died by $34.6 \pm 15$ minutes after cyanide exposure. Mean arterial pressure was significantly improved between the treatment and control group at study end $(p<0.0001)$. Blood lactate and oxygen saturation were also significantly improved between treatment and control at study end ( $p=0.0244$ and $p=0.0281$, respectively).

Conclusion: Oral administration of sodium thiosulfate improved survival and physiological parameters in a large animal model of oral cyanide toxicity.

002. Outbreak of Hypoglycemia from Counterfeit "V8" Male Sexual Enhancement Supplements

John W Downs ${ }^{1}$, Landon R Mueller ${ }^{1}$, Okey F Utah ${ }^{2}$, Brandon K Wills ${ }^{1}$, Kirk L Cumpston ${ }^{1}$, S. Rutherfoord Rose ${ }^{1}$

${ }^{I}$ Virginia Commonwealth University Health System, Richmond, VA, USA.

${ }^{2}$ Richmond City Health District, Richmond, VA, USA

Background: Over-the-counter male sexual enhancement supplements may contain undisclosed prescription pharmaceuticals such as phosphodiesterase5 inhibitors. For unclear reasons, other pharmaceutical contaminants may be present. This report describes an investigation of an outbreak of unexplained hypoglycemia associated with the use of an adulterated, counterfeit male sexual enhancement supplement called "V8."

Hypothesis: "V8" male sexual enhancement supplement contains a sulfonylurea contaminant resulting in symptomatic hypoglycemia.

Methods: This was an urgent prospective cohort study conducted as an outbreak investigation in conjunction with local and state public health authorities. Cases were defined as persons with unexplained hypoglycemia who reported use of "V8" male enhancement supplement. Cases were obtained through calls to the Virginia Poison Center. Case discovery and dissemination of information included health care facility notification, press release, and social media posts. Biologic samples and product samples were submitted to the Virginia Department of Health Laboratory for analysis for culprit contaminants. 
Results: Seven cases of unexplained symptomatic hypoglycemia associated with V8 male enhancement supplement use were reported to the Virginia Poison Center over a 4-week period. All cases were treated with supplemental dextrose and octreotide. All were admitted for a minimum of 24 hours of observation. Lowest recorded blood glucose concentrations ranged from 26 to $48 \mathrm{mg} / \mathrm{dL}$ (median $32 \mathrm{mg} / \mathrm{dL}$ ). Median hospital length of stay was 2 days. Product samples were obtained from retail locations as well as from cases. Product testing at the state public health laboratory confirmed glyburide estimated at $90-100 \mathrm{mg}$ per tablet, as well as sildenafil ranging 55-156 mg per tablet. Three cases had serum sulfonylurea screens performed and all three were positive for glyburide. No adverse effects of sildenafil were reported.

Conclusion: Counterfeit male sexual enhancement supplements contained prescription sildenafil and glyburide, which produced life-threatening hypoglycemia. These patients required admission and treatment of prolonged, refractory hypoglycemia with dextrose and supplemental octreotide.

\section{Assessment of the Overdosage Section of Labeling for Drugs Highly Associated with Drug Poison Fatalities}

\author{
Keith K Burkhart, Eric Brodsky \\ Food and Drug Administration, Silver Spring, MD, USA
}

Background: FDA regulations require the overdosage section of labeling for human prescription drugs be updated when new information becomes available that causes the labeling to become inaccurate, false, or misleading [21 CFR 201.57(c)(11) and 21 CFR 201.56(a)(2)].

Methods: We compared the overdosage management recommendations in the overdosage section of 29 selected labeling to overdosage management guidelines in medical toxicology textbooks and clinical toxicology practice guidelines. We selected representative labeling for drugs or drug classes associated with the most poisoning fatalities in 2017 in the National Poison Data System.

Results: In our review of selected labeling, 21 of 29 (72\%) overdosage sections provide overdosage management recommendations that are out of date and inconsistent with American Academy of Clinical Toxicology (AACT) recommendations. For example, many of these labeling have out-of-date elimination techniques such as emesis, gastrointestinal lavage, activated charcoal, cathartics, and forced diuresis. Additionally, 14 of 29 (48\%) overdosage sections have out-ofdate and potentially harmful antidote recommendations to manage overdosage-related hypotension, bronchospasm, and dysrhythmias. Some overdosage sections contained inaccurate, misleading, or incomplete information on the risk for significant toxicity in overdosed patients by mentioning initial overdose experience from a few patients. Some overdosage sections contained impractical and/or irrelevant data, such as lethal dose (LD50) data. Some overdosage sections did not include recommendations to contact a poison center or medical toxicologist. Comparisons of overdosage sections across drug classes in this analysis found some inappropriate inconsistent recommendations for overdosage management. We note that the overdosage sections of recently approved drugs include more up-todate overdosage management information.

Conclusion: The overdosage sections of labeling for a selected number of older drugs associated with overdosage-related fatalities contain outdated overdosage treatment recommendations. Application holders of drugs associated with overdosage should review the overdosage section of these labeling periodically to ensure they are up-to-date.

004. Rapid Toxin Elimination Achieved via an In Vitro Model of High-Volume Hemofiltration Within an Extracorporeal Membrane Oxygenation Circuit

Alison Grazioli ${ }^{1}$, Morgan Hoffman ${ }^{2}$, Daniel Hopkins ${ }^{2}$, Morgan Janes ${ }^{2}$, Milan Patel ${ }^{2}$, Manaahil Rao ${ }^{2}$, Zhongjun $\mathrm{Wu}^{2,3}$, Joshua King ${ }^{1,4}$, Helim Aranda-Espinosa ${ }^{2}$, Ronson Madathil ${ }^{3}$, Joseph Rabin ${ }^{3}$
${ }^{1}$ Department of Medicine, University of Maryland Medical School, Baltimore, MD, USA. ${ }^{2}$ University of Maryland A. James Clark School of Engineering, College Park, MD, USA. ${ }^{3}$ Department of Surgery, University of Maryland Medical School, Baltimore, MD, USA. ${ }^{4}$ Maryland Poison Center, Baltimore, MD, USA

Background: Extracorporeal removal of toxins is limited by the amount of blood which can be processed over a given time period. Extracorporeal membrane oxygenation (ECMO) processes blood roughly ten times faster than conventional hemodialysis. We recently demonstrated that highvolume hemofiltration in a patient undergoing ECMO is achievable, offering the possibility of markedly increased extracorporeal toxin elimination.

Hypothesis: A high-volume hemofiltration system within an ECMO circuit allows for superior extracorporeal toxin removal compared to conventional hemodialysis.

Methods: This is an in vitro single-compartment model of an ECMO circuit with an in-line high-volume hemofiltration system. A primary closed circuit with $5 \mathrm{~L}$ of $0.9 \%$ saline and $6.8 \mathrm{mg} / \mathrm{L}$ flecainide acetate (approximating a 5000-mg ingestion in an $80-\mathrm{kg}$ person) was connected to Revaclear@ hemodialysis filters attached in parallel. A centrifugal blood pump (CentriMag@) drove the primary circuit at a flow rate of $4 \mathrm{~L} /$ minute, while two separate pumps were used to remove effluent and infuse replacement fluid (saline). A trial with two hemodialysis filters was performed, followed by a second trial with eight filters. Flecainide concentration of samples drawn periodically was determined with ultraviolet-visible spectroscopy, and removal rates were calculated.

Results: In the two-filter circuit, effluent flow rate of $0.4 \mathrm{~L} /$ minute was achieved; flecainide concentrations decreased to $1.5 \mathrm{mg} / \mathrm{L}$ in 30 minutes, with peak removal rate of $115 \mathrm{mg}$ /hour. The eight-filter circuit achieved $1.87 \mathrm{~L} /$ minute effluent flow rate and flecainide levels decreased to $1.5 \mathrm{mg} / \mathrm{L}$ in 9 minutes (78\% decrease from initial concentration) with peak removal rate of $684 \mathrm{mg} /$ hour.

Conclusion: In an in vitro model of high-volume hemofiltration within an ECMO circuit, we show proof-of-concept of very high extracorporeal clearance. This system offers potential for both extremely rapid dialyzable toxin clearance and clinically significant removal of moderately lipophilic traditionally nondialyzable substances like flecainide. Further development of this system is ongoing.

\section{Day 1: Moderated Posters, Abstracts 005-010}

005. Trends in Emergency Department Tramadol Utilization, 20072016

Peter M Mullins ${ }^{1}$, Ali Pourmand ${ }^{2}$, Jeanmarie Perrone ${ }^{3}$, Lewis S Nelson ${ }^{4}$, Jesse M Pines ${ }^{5}$, Maryann Mazer-Amirshahi ${ }^{6}$

${ }^{1}$ Brigham and Women's Hospital, Boston, MA, USA. ${ }^{2}$ George Washington University, Washington, DC, USA. ${ }^{3}$ University of Pennsylvania, Philadelphia, PA, USA. ${ }^{4}$ Rutgers, Newark, NJ, USA. ${ }^{5}$ US Acute Care Solutions, Canton, OH, USA. ${ }^{6}$ MedStar Washington Hospital Center, Washington, DC, USA

Background: The prescription opioid epidemic has led to changes in opioid utilization patterns by providers, with an overall decrease in use for many opioids. There has been mounting concern that prescribers are increasingly utilizing tramadol, which has its own abuse potential and adverse effects, as an alternative to full-agonist opioids.

Research Question: To determine whether emergency department (ED) utilization of tramadol has increased over time.

Methods: We reviewed data from the National Hospital Ambulatory Medical Care Survey from 2007 to 2016 for adult ED visits in which tramadol was administered or prescribed. We explored patient demographics, hospital characteristics, resource utilization, and trends in the use of other opioids. 
Results: In 2007, tramadol was administered or prescribed during 1.55 million visits, accounting for $1.7 \%$ of ED visits. This increased to 4.6 million visits in 2016 ( $4.1 \%$ of ED visits, $p<0.001$ ), a relative increase of $141.2 \%$, while utilization of other opioids decreased from 20.7 to $18.8 \%$. Tramadol use increased for both sexes, but tramadol was used more commonly in women (61.2-63.8\% of tramadol visits). There were similar increases in the rate of tramadol use between white and non-white patients, although whites accounted for more visits overall. While tramadol use increased in all age groups, the largest increase was in patients age 55 to $64(450.0 \%, p<0.001)$. Tramadol use increased among all payer groups but increased more for visits with private insurance $(250 \%, p<0.001)$. Tramadol use increased in all areas of the USA except the Northeast, with the largest increases in the South $(184.6 \%)$ and West $(171.4 \%)$. The most common reasons for visit where tramadol was used were back pain (8.9\%), abdominal pain (7.3\%), and chest pain $(5.0 \%)$.

Conclusion: Despite evidence of limited efficacy, abuse potential, and adverse drug events, emergency medicine clinicians are increasingly utilizing tramadol, while use of other opioids is declining.

\section{Assessment of Hypertriglyceride Interference with Salicylate} Colorimetric Assay

Lauren T Murphy ${ }^{1,2}$, Caitlin M Kazmierczak ${ }^{1}$, John A Thompson ${ }^{1,2}$, Jennifer S Love ${ }^{1,2}$, Steven C Kazmierczak ${ }^{1}$

${ }^{1}$ Oregon Health \& Science University, Portland, OR, USA. ${ }^{2}$ Oregon Poison Center, Portland, OR, USA

Background: There are several case reports documenting patients with falsely elevated salicylate concentrations in the presence of hyperlipidemia. Interferences such as lipemia can be analyte-dependent, where the interference effect is dependent upon the concentration of analyte being tested, or analyteindependent where the interference effect is constant, regardless of the analyte concentration. The device at our institution claims that lipemia increases salicylate results by $19 \%$ at salicylate concentrations of $20 \mathrm{mg} / \mathrm{dL}$, but does not state whether this interference is analyte-dependent or analyte-independent.

Research Question: Does hypertriglyceridemia cause an analytedependent or analyte-independent interference effect with measurement of salicylate?

Methods: We tested the interference of hypertriglyceridemia by creating a $5 \times 7$ matrix of serum samples with varying concentrations of salicylate and lipid solution. We produced seven pools of serum with salicylate ranging in concentration from $1.7 \mathrm{mg} / \mathrm{dL}$ (below standard detection) to a treatable concentration of $42.4 \mathrm{mg} / \mathrm{dL}$. Each sample of salicylate was then divided into five pools with "Intralipid®" triglyceride reagent added, ranging from $150 \mathrm{mg} / \mathrm{dL}$ (serum baseline) to $7071 \mathrm{mg} / \mathrm{dL}$. We recorded salicylate concentration for each of the resulting 35 samples on the Siemens Vista 1500 utilizing the modified Trinder method.

Results: We found that hypertriglyceridemia causes a falsely elevated salicylate concentration in not only the serum with undetectable salicylate, but also in the samples containing larger concentrations of salicylate. The pool with $1.7 \mathrm{mg} / \mathrm{dL}$ triggered a positive, but therapeutic, concentration starting at a triglyceride concentration of $3500 \mathrm{mg} / \mathrm{dL}$. In samples containing salicylate, percent change in salicylate concentration increased from 0 to $476 \%$ with increasing triglycerides and was also dependent on baseline salicylate. The percent change in salicylate was most prominent in the pool with a low but therapeutic salicylate concentration $(6.9 \mathrm{mg} / \mathrm{dL})$.

Conclusion: Hypertriglyceridemia results in an analyte-dependent interference with salicylate concentration, falsely increasing results. This may lead to unnecessary treatment, further testing, and hospitalization of a patient.

\section{Kratom (Mitragyna speciosa) Liver Injury: a Scoping Review}

Jonathan Schimmel ${ }^{1}$, Richard C. Dart ${ }^{2}$

${ }^{1}$ Icahn School of Medicine at Mount Sinai, New York, NY, USA. ${ }^{2}$ Rocky Mountain Poison \& Drug Safety, Denver Health \& Hospital Authority, Denver, CO, USA
Background: Mitragyna speciosa ("kratom") leaves contain the mu opioid receptor partial agonists mitragynine and 7-hydroxymitragynine. In 2016, the United States DEA (Drug Enforcement Agency) declared its intention to list kratom as schedule I, but atypically postponed due to public pressure. The DEA and FDA (Food and Drug Administration) consider kratom under ongoing review, but there is a paucity of information regarding negative health effects. Although liver injury is cited as a potential health consequence, the same few case reports are repeatedly referenced without broader context. Furthermore, prior reports have largely lacked standardized methods of causality assessment. The objective is to evaluate the strength of causality in kratom liver injury, through a comprehensive review of human cases, and by reviewing epidemiologic, animal, and mechanistic reports that relate to kratom liver injury.

Methods: A scoping review was performed, using specific keywords, to identify human reports of potential kratom hepatotoxicity, and to further identify studies relating to animal reports, epidemiologic reports, and mechanistic studies. Hepatotoxicity causality was systematically examined by calculating RUCAM (Roussel Uclaf Causality Assessment Method) scores for case reports. Biopsy findings, potential pathophysiologic mechanisms, and management options are also discussed.

Results: A total of 21 cases were formally described, and many additional reports have been informally described in user forums. Of 19 cases that are scorable, modified RUCAM scores ranged from 1 to 8, mean 5.1, and median 5. Biochemically, the condition is heterogeneous or mixed, though histologically is predominantly cholestatic. Optimal management is unclear, and the majority have resolved with cessation alone.

Conclusion: Kratom likely causes liver injury based on the totality of low-quality human evidence in the form of case reports, FDA databases, and online user forums, and in the context of epidemiologic, animal, and mechanistic studies. It remains unclear which subgroups of users are at heightened risk.

008. Rattlesnake Envenomation in Arizona: Comparison of Patients Admitted to a Medical Toxicology Subspecialty Service Vs to Other Medical Specialists

Lauren M Porter ${ }^{1}$, Anne-Michelle Ruha ${ }^{2,3}$, Angela Padilla-Jones ${ }^{2,3}$, David Lee ${ }^{2}$, Allison Popa ${ }^{3}$, Richard Gerkin $\mathrm{Jr}^{2,3}$

${ }^{1}$ University Hospitals Center for Emergency Medicine, Cleveland, $\mathrm{OH}$, USA. ${ }^{2}$ University of Arizona College of Medicine-Phoenix, Phoenix, AZ, USA. ${ }^{3}$ Banner-University Medical Center Phoenix, Phoenix, AZ, USA

Background: Medical toxicologists (MTs) are trained in the evaluation and management of rattlesnake envenomation (RSB). Banner-University Medical Center (BUMCP) and Phoenix Children's Hospital (PCH) have the only medical toxicology admitting service in Arizona and care for approximately $50-70$ patients annually with RSB.

Hypothesis: We hypothesized that patients managed by MTs would have improved outcomes compared to patients admitted to non-MTs.

Methods: The Banner PCC is one of two AZ PCCs and covers Maricopa County. This is a retrospective review of Banner PCC records coded as snakebite between January 2, 2007, and December 31, 2017. Patients admitted to MTs were compared to those admitted to non-MTs. Data including demographics, clinical findings, labs, treatment, and outcomes were compared. Fisher exact tests, independent $t$ tests, and linear regression were used in the analysis.

Results: Seven hundred and forty-seven charts were identified. Three hundred and ninety-seven were lower extremity bites (54.1\%) vs upper extremity $(337,45.9 \%)$ and males outnumbered females 525 to $199(72.5$ vs $27.5 \%$ ). MTs cared for 543 patients primarily. There was no statistical difference between bite location (54.1\% LE, 49\% UE) as compared to those cared for by non-MTs (63.1\% LE vs $36.9 \%$ UE). LE bites had longer LOS (11 hours). Patients admitted to MTs had more swelling ( 94.6 vs $86.4 \%$ ) and coagulopathy (44.4 vs $24.6 \%$ ). They had more systemic effects (29.1 vs $26 \%$ ). Patients received less maintenance antivenom dosing when admitted to MTs (35 vs $72 \%$ ) with fewer adverse 
events (3.3 vs $8.9 \%$ ). Patients admitted to MT had shorter LOS (21 hours) with more consistent follow-up (91.3 vs $69.7 \%$ ).

Conclusion: Despite patients having increased swelling and coagulopathy, those cared for by MTs had a shorter LOS, more consistent follow-up, and fewer adverse events when being treated for rattlesnake envenomation.

\section{Lay Media Reports of Unintentional Opioid Exposure of Public Safety First Responders in North America}

Paul A Herman, Daniel S Brenner, Stewart Dandorf, Stephanie Kemp, Breann Kroll, Joshua Trebach, Yu-Hsiang Hsieh, Andrew Ian Stolbach Johns Hopkins School of Medicine, Baltimore, MD, USA

Background: Opioids do not spontaneously volatilize, transcutaneous absorption is very poor, and illness from unintentional exposure is extremely unlikely. We have characterized media reports of unintentional first responder opioid toxicity and attempted to verify whether there is any evidence to suggest that these represent true toxic exposures.

Methods: We queried LexisNexis for print and online articles on first responder opioid exposure published in North America between January 2012 and March 2018. Articles were reviewed by one of five reviewers who used a standard data collection form to determine if articles reported a case of emergency responder exposure. Data was abstracted including route of exposure, documented symptoms, and confirmatory testing. We calculated Fleiss' kappa statistic to determine the inter-rater agreement among five reviewers. Linear regression analysis was performed to determine the trend of number of opioid relevant or relevant exposure publications over time.

Results: The search yielded 1408 press publications, of which 214 reported a potential exposure. The Fleiss kappa was 0.92 for potential exposures. Linear regression analysis revealed a statistically significant increase in potential exposure articles. We found an increase in potential exposure publications of 9.2 articles per 6 months $(p=0.008)$. Of 214 reported exposures, none reported a plausible route of exposure, clinical manifestations consistent with opioid intoxication, or laboratory testing to confirm exposure.

Conclusion: Although first responders should continue to take reasonable precautions, analysis of these media reports is consistent with the consensus of the medical community that unintentional poisoning is extremely unlikely.

010. Assessing the Impact of Legislative Changes on the Rates of Acetaminophen-Induced Hepatotoxicity

Michael Levine ${ }^{1}$, Evan S Schwarz ${ }^{2}$, Anthony Pizon ${ }^{3}$, Timothy Wiegand ${ }^{4}$, Brandon Wills ${ }^{5}$, Alicia Minns ${ }^{6}$, Ilene A Claudius ${ }^{7}$, Tony Gao ${ }^{6}$, Tyler Falter ${ }^{4}$, Shirley Shao ${ }^{3}$

${ }^{1}$ University of Southern California, Los Angeles, CA, USA. ${ }^{2}$ Division of Medical Toxicology, Department of Emergency Medicine, Washington University, St. Louis, MO, USA. ${ }^{3}$ Division of Medical Toxicology, Department of Emergency Medicine, University of Pittsburgh, Pittsburgh, PA, USA. ${ }^{4}$ Division of Medical Toxicology, Department of Emergency Medicine, Rochester University, Rochester, NY, USA. ${ }^{5}$ Division of Medical Toxicology, Department of Emergency Medicine, Virginia Commonwealth University, Richmond, VA, USA. 'Division of Medical Toxicology, Department of Emergency Medicine, University of California, San Diego, San Diego, CA, USA. ${ }^{7}$ Department of Emergency Medicine. Harbor UCLA Medical Center, Torrance, CA, USA. ${ }^{8}$ Department of Emergency Medicine, University of Southern California, Los Angeles, CA, USA. ${ }^{9}$ Department of Emergency Medicine, Weill/Cornell Medical College in Qatar

Background: Acetaminophen is one of the most common causes of acute hepatic failure in the USA. In 2014, several legislative and regulatory changes surrounding acetaminophen occurred, including a rescheduling of acetaminophen-hydrocodone from a class III drug to a class II drug, along with limiting the maximal daily dose from 4 to $3 \mathrm{~g}$. The impact of such changes has not been evaluated.

Methods: This study is a retrospective study of all cases of acetaminophen-induced hepatic injury from six tertiary-care medical centers, each with active liver transplant and medical toxicology services. Cases presenting between 2011 and 2013 were compared with those occurring between 2015 and 2017. The patterns prevalence and specific patterns of liver injury were compared between these two time periods. Results: There was a decline in the overall number of acetaminophenassociated liver injury between the two periods ( 278 pre-2014 vs. 223 post-2014). There was a decline in the number of acetaminophenhydrocodone cases in these two periods (60 vs. $30 ; p=0.018$ ). This decline was not associated with other opioids, as there was no change in the number of cases of acetaminophen-induced liver injury involving acetaminophen-oxycodone. However, there was an increase in the cases of isolated acetaminophen-induced liver injury (acetaminophen without opioid) pre-2014 to post-2014 periods (OR 1.73; 95\% CI 1.2-2.5).

Conclusion: Comparing the pre-2014 era to the post-2014 era, there was a decline in the number of cases of liver injury due to acetaminophenhydrocodone, an increase in the number of cases from acetaminophen without opiate, and no change in the number of cases of liver injury due to acetaminophen-oxycodone.

\section{Day 1: Posters, Abstracts 011-058}

011. Methemoglobinemia Due to Topical Dapsone Use in a Slow Acetylator

Dominique D Diggs ${ }^{1}$, Meagan M Ladell ${ }^{1}$, Matthew T Stanton ${ }^{2}$, Andrew $\mathrm{N}_{\text {Farkas }}^{2}$

${ }^{I}$ Children's Wisconsin, Milwaukee, WI, USA. ${ }^{2}$ Wisconsin Poison Center, Milwaukee, WI, USA

Background: While oral dapsone is commonly associated with acquired methemoglobinemia, topical dapsone is a much rarer cause of this condition. $\mathrm{N}$-acetylation and $\mathrm{N}$-hydroxylation are major pathways of dapsone metabolism.

Methods: This is a single case report. A 16-year-old girl presented to a tertiary pediatric emergency department with progressive headache, nausea, and confusion over several days. She initially denied any new medications, ingestions, or other exposures. Vital signs included pulse 140, respiratory rate 36 , and oxygen saturation $75-88 \%$ on room air, and she exhibited cyanosis which did not improve with supplemental oxygen.

Results: Laboratory testing revealed a methemoglobin level of $34.1 \%$. Upon further discussion, the patient reported that she was prescribed topical dapsone gel $(7.5 \%) 3$ weeks prior to treat facial acne and had been using the cream on her face, trunk, and back. The patient's methemoglobin level improved to $13.7 \%$ after receiving $100 \mathrm{mg}$ of methylene blue. She received a second dose 48 hours later when her level rose to $16.1 \%$, with subsequent resolution of toxicity. A serum dapsone level was $13 \mathrm{mcg} / \mathrm{mL}$ (normal $0.1-7 \mathrm{mcg} / \mathrm{mL}$ ). Further testing with liquid chromatography revealed a monoacetyldapsone:dapsone ratio of 0.10 (normal > 0.35 ), consistent with the slow acetylation phenotype.

Conclusion: The use of topical medications may be underreported by patients, highlighting the need for clinicians to specifically elicit this history when methemoglobinemia or other toxicities are suspected. Additionally, there is a theoretical basis for the slow acetylation phenotype to increase the risk of adverse effects of dapsone via shunting to the hydroxylamine metabolite which may generate more oxidant stress; however, there is a lack of clinical data to prove this association. As the understanding of pharmacogenomics increases, screening for the slow acetylation phenotype could become a useful strategy for identifying patients in whom alternatives to dapsone and other medications should be considered. 
012. Fomepizole Levels in Ethylene Glycol Poisoned Pediatric Twins Treated with CRRT

Michael A Hayoun ${ }^{1}$, Michael S Filigenzi ${ }^{2}$, Stephanie Nguyen ${ }^{1}$, James A Chenoweth $^{1}$, Jonathan B Ford ${ }^{1}$

${ }^{1}$ UC Davis Health, Sacramento, CA, USA. ${ }^{2}$ California Animal Health and Food Safety Laboratory at UC Davis, Davis, CA, USA

Background: It is well established that fomepizole (4MP) dosing changes during high flux hemodialysis in ethylene glycol (EG)-poisoned patients. Little data is available for dosing in lower flux continuous renal replacement therapy (CRRT), even more sparse for the pediatric population.

Methods: This is a retrospective patient chart review of a pair of EGpoisoned identical twins treated at two different PICUs. A pair of 7month-old twin females were poisoned with EG and found to be critically ill, requiring dialysis. One twin was kept at the sending PICU and managed remotely by the toxicology team, and one was transferred to the academic medical center and was seen in person. Both twins were treated with 4MP every 12 hours as well as CRRT. One twin was treated using a Next Stage CAR-125 and the other using the Prismaflex system. Both had varying blood flow rates. Blood samples used for the routine care of the patients were analyzed for 4MP levels using GCMS. Analyte stability testing is still in process.

Results: There is a trend toward therapeutic 4MP levels $(10-20 \mathrm{mg} / \mathrm{L})$ in the samples that were analyzed within 8 hours of dosing and at lower flux defined as blood flow rates of $30 \mathrm{~mL} /$ minute.

Conclusion: 4MP should be given more often in patients undergoing CRRT. Every 8 hours is suggested by this small sample, but further study is required before making firmer recommendations.

\section{Osmolal Gap: an Inaccurate Test}

Ryan Marino ${ }^{1,2}$, Alexander Sidlak ${ }^{3}$, Anthony Pizon $^{3}$

${ }^{1}$ University Hospitals, Cleveland, OH, USA. ${ }^{2}$ Case Western Reserve University School of Medicine, Cleveland, OH, USA. ${ }^{3}$ University of Pittsburgh School of Medicine, Pittsburgh, PA, USA

Background: Ethanol is the most common cause of osmolal gap elevations despite attempts to correct the osmolality formula for this contribution. Current formulas still do not accurately reflect ethanol's contribution to the osmolal gap, presenting a clinical dilemma.

Hypothesis: Available formulas for calculating the osmolal gap are inaccurate in acute ethanol intoxication.

Methods: We conducted a prospective cohort study of 22 healthy volunteers who were given weight-based doses of ethanol over 2 hours in order to achieve an ethanol level greater than $200 \mathrm{mg} / \mathrm{dL}$. Laboratory testing was performed concurrently at $0,2,4$, and 6 hours, including serum levels of ethanol, osmolality, sodium, blood urea nitrogen, and glucose. Measured osmolality using freezing-point depression was compared with calculated osmolality using the standard formula $(2 \times[\mathrm{Na} \mathrm{mOsm} / \mathrm{L}]+[\mathrm{BUN} \mathrm{mg} / \mathrm{dL}]$ $2.8+$ [glucose $\mathrm{mg} / \mathrm{dL}] / 18+[\mathrm{ETOH} \mathrm{mg} / \mathrm{dL}] / 4.6$ ). Osmolal gap $>10 \mathrm{mOsm} /$ $\mathrm{kg}$ or $<-10 \mathrm{mOsm} / \mathrm{kg}$ was considered abnormal.

Results: Four of the 22 subjects (18\%) from a healthy, representative sample had an osmolal gap at an ethanol level of $0 \mathrm{mg} / \mathrm{dL}$. Of the 66 other measurements with positive ethanol levels, there were 14 false osmolal gaps (21\%) using the standard formula with 4.6 to represent ethanol's molecular weight and 7 false osmolal gaps (13\%) when the 4 subjects with baseline gaps were removed. Using the most commonly accepted correction factor derived by Purssell et al. of 3.7, there were 31 false positives out of 66 samples (47\%), with 28 false positives (52\%) after removing the 4 subjects with baseline gaps. Conclusion: Calculated osmolal gap is a screening tool for toxic alcohol ingestions with costly therapies often initiated based on elevations of osmolal gap alone. These data show that osmolal gap is an inaccurate test with a high false positive rate both at baseline and in acute ethanol ingestion.
014. Single Versus Continued Dosing of Fomepizole During Hemodialysis for Ethylene Glycol Poisonings

Alexander M Sidlak ${ }^{1}$, James P Van Meerbeke ${ }^{1}$, Ryan T Marino ${ }^{2}$, Anthony F Pizon ${ }^{1}$

${ }^{1}$ School of Medicine, University of Pittsburgh, Pittsburgh, PA, USA.

${ }^{2}$ Case Western Reserve School of Medicine, Pittsburgh, PA, USA

Background: In cases of ethylene glycol (EG) toxicity requiring hemodialysis (HD), fomepizole is dosed every 4 hours. Given that HD efficiently clears EG's toxic metabolites, it is unclear if multiple doses (MD) of fomepizole improve patient outcomes or whether a single dose (SD) prior to initiation of HD is sufficient.

Hypothesis: Single-dose fomepizole for EG toxicity requiring hemodialysis is noninferior to repeated dosing.

Methods: We retrospectively reviewed cases of EG toxicity at a large academic institution from 1998 to 2018. Patients with EG levels greater than $20 \mathrm{mg} / \mathrm{dL}$ treated with HD were included. Baseline patient characteristics, creatinine at discharge, hospital length of stay (LOS), and complications were collected. We compared patients who received single doses of fomepizole prior to HD to those receiving continued dosing.

Results: Twenty-four patients were identified (20 in the MD group and 4 in the SD group). Median EG level was $170 \mathrm{mg} / \mathrm{dL}$ (IQR 65-270) in the MD group and $560 \mathrm{mg} / \mathrm{dL}$ (IQR 170-1800) in the SD group. Degree of acidosis (pH 7.1 vs. 7.1) and initial creatinine (1.6 vs. $1.5 \mathrm{mg} / \mathrm{dL}$ ) were similar between the two groups. There was one death in the multidose group and none in the single-dose group. Creatinine at the time of discharge was no different between the two groups ( 3.7 vs. $2.2 \mathrm{mg} / \mathrm{dL}, p=$ NS, Student's $t$ test). Hemodialysis was continued in 7/19 (MD) vs. 1/4 (SD) patients upon discharge. LOS was similar between groups (7.6 [95\% CI 5.3-9.9] vs. 6.6 days [4.4-8.8]).

Conclusion: In this retrospective study, patients with acute EG toxicity requiring hemodialysis treated with a single dose of fomepizole had similar outcomes to those receiving continued dosing of fomepizole. This raises the possibility that only a single dose of fomepizole is needed in acute ethylene glycol toxicity if hemodialysis is initiated. Additional prospective and randomized studies are needed.

\section{Delayed Ethanol Absorption and Zero-Order Elimination After Infant Ethanol Exposure}

Alexander M Sidlak, Shirley Shao, Joshua A Shulman
University of Pittsburgh School of Medicine, Pittsburgh, PA, USA

Background: Ethanol toxicity in infants is rare. Prior case series have shown similar and occasionally faster rates of ethanol metabolism in children despite lower levels of alcohol dehydrogenase and cytochrome P450 isoform 2E1 (CYP2E1). No previous case reports have demonstrated prolonged gastric emptying or delayed absorption.

Hypothesis: Despite being alcohol naïve, infants have similar rates of metabolism of ethanol. Toxicokinetics may differ from delayed absorption.

Methods: This is a single patient case report. A 9-month-old boy $(9.6 \mathrm{~kg})$ presented after accidental ethanol ingestion. The child's teenage sister made a 6-ounce bottle for the child, accidentally mixing the formula with vodka $40 \%(\mathrm{v} / \mathrm{v})$, which had been stored in a water bottle. An hour later, the child exhibited psychomotor slowing and the child was taken to a local ED. An initial alcohol level was $285 \mathrm{mg} / \mathrm{dL}, 230$ minutes after ingestion. Serial ethanol levels were obtained during the child's admission.

Results: Serum ethanol level 10 hours after ingestion was $325 \mathrm{mg} / \mathrm{dL}$. The patient did not develop metabolic acidosis, hepatotoxicity, hypoglycemia, pancreatitis, or electrolyte derangements. After clearance from the child advocacy service, they were discharged home on hospital day 3 . Cases of ethanol toxicity in infants are rare. Levels as high as $575 \mathrm{mg} / \mathrm{dL}$ have been reported without mortality. Though multisystem organ 
dysfunction can occur, our patient did not develop any clinical sequelae from ingestion. They metabolized the alcohol with zero-order kinetics at a rate of $22.34 \mathrm{mg} / \mathrm{dL}$. Curiously, their level continued to rise more than 10 hours after initial ingestion. We hypothesized that this was secondary to delayed absorption and ethanol-induced pylorspasm.

Conclusion: Ethanol ingestion in infants is rare and may not necessarily lead to severe sequelae. Metabolism is similar to adults despite naivety to ethanol, but absorption may be delayed, prolonging the duration of toxicity.

\section{Progression to Delirium Tremens Despite High-Dose Phenobarbital Taper}

Alexander M Sidlak ${ }^{1}$, Jordan L Singer ${ }^{1}$, Ryan T Marino ${ }^{1,2}$, Anthony T Scoccimarro ${ }^{1}$, Shirley Shao ${ }^{1}$, Michael G Abesamis ${ }^{1}$

${ }^{1}$ School of Medicine, University of Pittsburgh, Pittsburgh, PA, USA.

${ }^{2}$ School of Medicine, Case Western University, Cleveland, OH, USA

Background: Many hospitals have instituted fixed-dose phenobarbital tapers to prevent the development of alcohol withdrawal. Regimens differ, but generally, they include a weight-based loading dose followed by a standardized taper.

Hypothesis: Fixed-dose phenobarbital can fail to prevent progression of alcohol withdrawal in select patients.

Methods: We reviewed patients managed by our Toxicology service over the last 2 years that were diagnosed with delirium tremens (DTs). All patients who had a phenobarbital taper initiated in the first 24 hours of admission were included. In this case series, four patients were identified who received the phenobarbital taper for alcohol withdrawal. The mean initial dose of intramuscular phenobarbital prior to the development and identification of delirium tremens (DTs) was $394.75 \mathrm{mg}$ [205-560 mg]. DTs were identified by bedside evaluation by a medical toxicologist. After identification, phenobarbital was discontinued, and patients were treated with high-dose symptom-triggered intravenous diazepam with improvement of their withdrawal. In a single case, a ketamine infusion was required for adjunctive treatment. A mean dose of $381.35 \mathrm{mg}$ [65$600 \mathrm{mg}$ ] of diazepam was given after patients had deteriorated.

Conclusion: In this case series, escalating doses of benzodiazepines were needed despite patients receiving loading doses of phenobarbital. Institutionalized protocols for phenobarbital tapers may not reliably prevent the development of severe alcohol withdrawal. Factors leading to failure may include a failure to identify early symptoms of alcohol withdrawal and unpredictable kinetics with intramuscular dosing. More importantly, a therapeutic-clinical mismatch can occur with tapering regimens: decreasing $\mathrm{GABA}_{\mathrm{A}}$ agonist therapy is being given despite worsening symptoms. Early initiation of symptom-triggered management may allow for earlier recognition of the progression of symptoms and more appropriate treatment of severe alcohol withdrawal.

\section{A Retrospective Analysis of Serotonin Toxicity with Bupropion Overdose}

Karl O Kovisto ${ }^{1}$, Joshua A Shulman ${ }^{1}$, Ryan T Marino ${ }^{2}$, Michael G Abesamis $^{1}$, Alexander M Sidlak ${ }^{1}$

${ }^{1}$ University of Pittsburgh School of Medicine, Pittsburgh, PA, USA. ${ }^{2}$ Case Western Reserve University School of Medicine, Cleveland, OH, USA

Background: Bupropion is a synthetic cathinone similar to "bath salts." Therapeutically, the drug is a reuptake inhibitor of norepinephrine and dopamine. Bupropion has not historically been thought to lead to serotonin syndrome; however, animal studies show increased serotonin receptor activation with high doses of bupropion, and a recent case report described the development of severe serotonin syndrome in a teenager after isolated bupropion overdose.

Hypothesis: The incidence of serotonin toxicity from bupropion overdoses is higher than reported.
Methods: This is a retrospective study of all patients with a bupropion overdose admitted to our toxicology service between 2015 and 2017. Patients who overdosed on other serotonergic medications were excluded. A diagnosis of serotonin toxicity was determined retrospectively using Hunter criteria.

Results: Overall, 96 patients were identified who had possible bupropion toxicity. Of these, 18 patients ingested bupropion in the absence of other serotonergic drugs. Of these 16 patients, $33 \%$ had clonus, $100 \%$ had tachycardia (HR $>100), 50 \%$ had altered mental status or agitation, $56 \%$ had hyperreflexia, $22 \%$ had a tremor, $6 \%$ had a wide QRS (> $120 \mathrm{~ms}$ ), and $22 \%$ had a QTc $>500 \mathrm{~ms} ; 44 \%$ of these patients met criteria for serotonin syndrome. The incidence of serotonin toxicity among those ingesting bupropion in a suicide attempt $(5 / 8)$ was higher than those with an accidental ingestion (1/3) or recreational use (2/8). The average dose of bupropion ingested was $2400 \mathrm{mg}$.

Conclusion: In our 2-year retrospective analysis, $44 \%$ of patients presenting after a bupropion overdose without co-ingestion of other serotonergic drugs developed serotonin toxicity. Prior observational studies may underestimate the rate of serotonin toxicity because of a lack of bedside toxicologist evaluation or incomplete data entry into national databases. This should prompt clinicians to monitor for signs of serotonin toxicity when evaluating patients with a bupropion overdose.

018. Comparison of the Australian and New Zealand Referral Criteria Versus the King's College Criteria to Predict Mortality or Liver Transplant in Acetaminophen Overdose

Joyce $\mathrm{Xu}^{1}$, Adam Testro ${ }^{2}$, Anselm Wong ${ }^{2,3}$

${ }^{1}$ University of Melbourne, Parkville, VIC, Australia. ${ }^{2}$ Austin Health, Heidelberg, VIC, Australia. ${ }^{3}$ Monash University and University of Melbourne, Melbourne, VIC, Australia

Background: Acetaminophen overdose can lead to fulminant hepatic failure. The King's College criteria (KCC) is the most widely used survival predicting model. The Australia and New Zealand (ANZ) referral criteria incorporates several markers that have not been studied for its predictive value.

Research Question: How does the ANZ referral criteria compare to the $\mathrm{KCC}$ for predicting mortality or need for liver transplant from acetaminophen overdose.

Methods: This is a retrospective analysis of patients presenting to the Austin Hospital between 2010 and 2019 with acetaminophen overdose requiring treatment with $N$-acetylcysteine. We evaluated 983 acetaminophen overdose cases from 2010 to March 2019. The primary outcome was death or transplant. Sensitivity and specificity, along with associated ROC curves, were determined for both models to predict the primary outcome.

Results: A total of 481 cases met inclusion criteria. Eighteen cases $(3.7 \%)$ met the composite endpoint of death or transplant. The ANZ referral criteria has a higher sensitivity $(100 \%, 95 \%$ CI $81.5,100)$, but lower specificity $(88.3 \%, 95 \%$ CI $85,91.1)$ than the KCC. The ROC AUC for the $\mathrm{KCC}$ is 0.868 (95\% CI $0.760,0.977$ ), and the ROC AUC for the ANZ referral criteria is 0.627 (95\% CI 0.547, 0.707). Cohen's kappa was calculated to be 0.449 , showing moderate agreement between the KCC and ANZ referral criteria. On logistic regression, after backward stepwise elimination, the final regression model for the KCC included three variables: serum creatinine $>300 \mathrm{mmol} / \mathrm{L}$, persistent acidosis with a $\mathrm{pH}<$ 7.3, and high-grade encephalopathy. The final model for ANZ criteria included serum creatinine $>200 \mathrm{mmol} / \mathrm{L}$, persistent acidosis with $\mathrm{pH}<$ 7.3 , and systolic hypotension.

Conclusion: The ANZ referral criteria compared to the KCC was more sensitive for the outcome of mortality and transplant. This is important for screening patients that may become unstable and difficult to transfer at a later stage of their admission.

\section{Abstract Withdrawn}


020. Hypothermia-Associated Prolonged Paralysis After Rapid Sequence Intubation with Rocuronium

Anita Mudan ${ }^{1,2}$, Jacob A Lebin ${ }^{1,2}$, Craig G Smollin ${ }^{1,2}$

${ }^{1}$ Department of Emergency Medicine, University of California San Francisco, San Francisco, CA, USA. ${ }^{2}$ California Poison Control System, San Francisco Division, San Francisco, CA, USA

Background: Rocuronium is a paralytic used in rapid sequence intubation. Several studies have reported prolonged paralytic effects in hypothermic patients on continuous infusions of nondepolarizing agents, but similar reports after single rapid sequence intubation dosing remain rare. We report a case of prolonged paralysis after a single dose of rocuronium in a hypothermic patient.

Methods: This is a single patient chart review. A 63-year-old woman with hypertension, insulin-dependent diabetes, and chronic obstructive pulmonary disease presented after a cardiac arrest. In the emergency department, she was moving all extremities, but not following commands, and was intubated for airway protection with etomidate $20 \mathrm{mg}$ and rocuronium $100 \mathrm{mg}$. Shortly after intubation, the patient became profoundly hypotensive, which persisted despite hydrocortisone, calcium gluconate, and escalating doses of norepinephrine, epinephrine, and vasopressin. Labs were notable for creatinine $1.67 \mathrm{mg} / \mathrm{dL}$ and lactate $5.4 \mathrm{mmol} / \mathrm{L}$. The patient was admitted to the intensive care unit and was noted to be hypothermic to $32.9^{\circ} \mathrm{C}$, but was not intentionally cooled. After several hours off of sedation and normalization of her temperature, she remained unresponsive to noxious stimuli with absent brainstem reflexes. Seven hours after intubation, sugammadex $370 \mathrm{mg}$ was administered with immediate improvement in neurological status and hemodynamics. She was discharged 4 days later with no neurological sequelae. Discussion: Rocuronium is a nondepolarizing paralytic that acts by blocking acetylcholine from binding to receptors on the motor end plate. In hypothermia, the rate of rocuronium elimination is reduced, which prolongs the duration of detectable plasma concentrations. Studies of continuous infusions have shown that a decrease in body temperature of $2{ }^{\circ} \mathrm{C}$ can double the duration of neuromuscular blockade, but this has been rarely reported following rapid sequence intubation.

Conclusion: Hypothermia can significantly prolong the effects of paralytic agents, even after single bolus dosing.

\section{2,4-Dinitrophenol Ingestion Resulting in Acute Heart Failure} and Hyperthermia

Priya Srihari, Richard F Clark

University of California, San Diego, San Diego, CA, USA

Background: 2,4-Dinitrophenol (DNP) has been used as a weight loss supplement and is readily available online. It causes weight loss by uncoupling oxidative phosphorylation to increase fat metabolism. We present a nonfatal case of chronic ingestion of DNP resulting initially in heart failure.

Methods: This is a single case report. A 32-year-old male presented with chest pain. Electrocardiogram showed ST elevation. He reported ingesting $250 \mathrm{mg}$ of DNP and $100 \mathrm{mcg}$ of levothyroxine daily for 910 days for weight loss, which he had stopped taking 2 days prior to presentation. He was initially normothermic. He had an elevated troponin and global hypokinesis with decreased ejection fraction (EF) on bedside echocardiogram. On day 2, he became hyperthermic and was intubated, paralyzed, and treated with cooling measures. Thyroid studies suggested that presentation was not related to levothyroxine ingestion. On day 4 , the patient was extubated.

Results: Initial troponin T was $640 \mathrm{ng} / \mathrm{L}$ (normal $<22 \mathrm{ng} / \mathrm{mL}$ ). ED echocardiogram demonstrated global hypokinesis and an EF of 20-30\%. Formal echocardiogram the next day showed an EF of $45 \%$. The patient was intubated and briefly paralyzed to facilitate cooling. Maximum temperature was $40{ }^{\circ} \mathrm{C}$. Maximum troponin was $1052 \mathrm{ng} / \mathrm{mL}$. Thyroid studies showed a TSH of $0.04 \mu \mathrm{IU} / \mathrm{mL}(0.27-4.20 \mu \mathrm{IU} / \mathrm{mL})$, free T4 of $1.15 \mathrm{ng} /$ dL (0.93-1.70 ng/dL), total T3 of $0.8 \mathrm{ng} / \mathrm{mL}(0.8-2.0 \mathrm{ng} / \mathrm{mL})$, thyroglobulin of $12.6 \mathrm{ng} / \mathrm{mL}(1.3-31.8 \mathrm{ng} / \mathrm{mL})$, and thyroglobulin antibody of $<0.9 \mathrm{IU} / \mathrm{L}$, suggesting that presentation was not due to surreptitious thyrotoxicosis. Troponins declined and the patient was normothermic at discharge.

Discussion: DNP causes uncoupling of oxidative phosphorylation and can result in hyperthermia, diaphoresis, tachycardia, and tachypnea, and in large ingestions, cardiovascular collapse. Heart failure has not previously been reported with DNP ingestion.

Conclusion: Chronic DNP ingestion can result in cardiomyopathy.

\section{Neuroleptic Malignant Syndrome After Depot Injection of} Zuclopenthixol

Priya Srihari, Sam T Ontiveros, Bryan Corbett, Shaun D Carstairs University of California, San Diego, San Diego, CA, USA

Background: Zuclopenthixol is a thioxanthene antipsychotic that is a D1and D2-receptor antagonist. It is not approved for use in the USA but is commonly used in other countries. We present a case of depot injection of zuclopenthixol resulting in neuroleptic malignant syndrome (NMS).

Methods: This is a single patient chart review. A 58-year-old female with a history of early onset Alzheimer's disease was evaluated by a physician in Mexico for a 2-month history of worsening aggression; she was administered a 200-mg intramuscular depot injection of zuclopenthixol. She was not taking other antipsychotics at the time. Shortly thereafter, she developed mutism, obtundation, and severely decreased movement. At baseline, the patient was able to converse, walk, and perform activities of daily living. Three days prior to our evaluation, she developed hypersalivation and was transferred to our facility in the USA. On day 5 at our facility (approximately 20 days after zuclopenthixol injection), the toxicology service was consulted due to persistent depressed mental status. Results: On examination, the patient was nonverbal and demonstrated diffusely slowed movement and cogwheel rigidity in her bilateral upper extremities. Vital signs including temperature were within normal limits. Laboratory studies were significant for an elevated creatine phosphokinase of $448 \mathrm{U} / \mathrm{L}$ (normal $0-175 \mathrm{U} / \mathrm{L}$ ) and an elevated white blood cell count of 12,400 cells $/ \mathrm{mm}^{3}$. CT of the head and MRI of the brain showed global volume loss thought to be due to Alzheimer's disease. She was started on bromocriptine and, shortly thereafter, demonstrated improvement in her mental status and mobility. Given the onset of symptoms shortly after depot injection of zuclopenthixol and improvement after bromocriptine administration, the patient's presentation was thought to be induced by zuclopenthixol administration.

Conclusion: Intramuscular injection of zuclopenthixol can result in the development of neuroleptic malignant syndrome.

\section{Massive Nonfatal Pediatric Ingestion of Hydroxychloroquine}

Priya Srihari, Han T Gao, Allyson A Kreshak

University of California, San Diego, San Diego, CA, USA

Background: Hydroxychloroquine is a 4-aminoquinoline used to treat malaria and inflammatory diseases. In toxicity, it can cause arrhythmia and cardiovascular collapse. Here we present a massive hydroxychloroquine ingestion in a pediatric patient who survived with early intubation, electrolyte repletion, and high-dose epinephrine and diazepam infusions.

Methods: This is a single patient chart review. A 15-year-old female presented after an intentional ingestion of approximately $10-11 \mathrm{~g}$ of hydroxychloroquine. On arrival to the emergency department (ED), she was awake with vital signs showing heart rate 154 beats per minute and blood pressure (BP) 106/68 mmHg. Shortly after, she became hypotensive to BP $69 / 39 \mathrm{mmHg}$. Poison control was contacted. An urgent call back placed by the consulting toxicologist advocated for early and aggressive care. The patient was then intubated and started on high-dose epinephrine and diazepam 
infusions. Prompt electrolyte repletion was initiated, and she was transferred to the regional children's hospital.

Results: Initial ED laboratory studies were significant for potassium $3.0 \mathrm{mmol} / \mathrm{L}$ (normal 3.4-5.0 $\mathrm{mmol} / \mathrm{L}$ ). After transfer to the pediatric intensive care unit (PICU), repeat labs showed serum hydroxychloroquine level of $13,000 \mathrm{ng} / \mathrm{mL}$ (reporting limit $>100 \mathrm{mg}$ ) and potassium of $2.8 \mathrm{mmol} / \mathrm{L}$ (normal 3.5-5.5 mmol/L). Her electrocardiogram was significant for a QRS of $102 \mathrm{~ms}$ and QTc of $575 \mathrm{~ms}$. A second hydroxychloroquine level at 24 hours post-ingestion was $5600 \mathrm{ng} / \mathrm{mL}$. An arterial line was placed for BP monitoring and a central line was placed to continue electrolyte repletion. Twentyfour hours after PICU presentation, her BP began to improve, and her infusions were titrated down and discontinued. On hospital day 3 , she was successfully extubated, and at time of discharge, her cardiovascular status was normal, and she made a full recovery.

Conclusion: Massive hydroxychloroquine ingestions can be successfully treated with early aggressive supportive care.

\section{Intravenous Lipid Emulsion Therapy for Recurrent Cardiac Toxicity in an Intentional Diphenhydramine Overdose}

Carolyn E Fox ${ }^{1}$, Michael C Beuhler ${ }^{2}$, Lauren Piper ${ }^{1}$, Kartik Shah ${ }^{1}$, AnnJeannette Geib ${ }^{1}$

${ }^{1}$ Atrium Health's Carolinas Medical Center, Charlotte, NC, USA. ${ }^{2}$ North Carolina Poison Control, Charlotte, NC, USA

Background: Diphenhydramine is known to cause sodium channel blockade in overdose leading to wide-complex tachycardias. There has not been reported recurrent diphenhydramine toxicity following clinical resolution from a single ingestion.

Hypothesis: Diphenhydramine can cause recurrent cardiac toxicity in large overdose, and intravenous lipid emulsion (ILE) may be beneficial. Methods: This is a single patient chart review of a 17-year-old female who intentionally ingested approximately 280 tablets diphenhydramine $25 \mathrm{mg}$ (total $\approx 7 \mathrm{~g})$. She had multiple seizures and required intubation and sedation. She was given activated charcoal within 4 hours. Her cardiac rhythm oscillated between sinus rhythm, SVT with aberrancy, widecomplex arrhythmias, and supraventricular bigeminy. She responded well to sodium bicarbonate and electrolyte repletion and, at approximately 24 hours after ingestion, was extubated, awake, and lucid with normal sinus rhythm. At 32 hours after ingestion, she began to have ectopic, nonperfusing beats. At 45 hours, she developed hypotension, which was treated with norepinephrine, and an intermittent wide-complex ventricular tachycardia which was refractory to sodium bicarbonate, chemical overdrive pacing, and lidocaine. She was given aggressive potassium and magnesium repletion. She was then given ILE and the norepinephrine was discontinued which improved her rhythm within minutes of administration. She continued to have QTc prolongation until 6 days after ingestion. She gradually improved, was weaned from support, and discharged without sequelae.

Results: A urine diphenhydramine level (LC-MS/MS) on hospital day 4 was $240 \mathrm{ng} / \mathrm{mL}$. The patient and her mother confirmed single ingestion of diphenhydramine without coingestants during her lucid interval and at discharge.

Conclusion: This case demonstrates delayed, recurrent, and refractory cardiac dysrhythmias at greater than 24 hours after a single ingestion of diphenhydramine following a lucid interval with normal cardiac rhythm. ILE may be considered in large diphenhydramine overdoses with dysrhythmias that are refractory to bicarbonate and electrolyte repletion.

\section{Treatment of Beta Blocker and Calcium Channel Antagonist Poisoning by Medical Toxicologists}

Kevin T Baumgartner, Evan S Schwarz, On Behalf of the ToxIC Investigators Consortium (ToxIC)

Washington University School of Medicine, Saint Louis, MO, USA
Background: Beta blockers (BB) and calcium channel antagonists (CCA) are potentially lethal in overdose. Multiple therapies are used to treat $\mathrm{BB}$ and $\mathrm{CCA}$ poisoning.

Research Question: What therapies do medical toxicologists use to treat $\mathrm{BB}$ and CCA poisoning?

Methods: This is a retrospective review of case data from the Toxicology Investigators Consortium (ToxIC) database. ToxIC was queried for cases involving any BB or CCA reported from January 2017 to August 2019. Cases were excluded if they involved chronic poisonings or if signs and symptoms were considered unlikely to be related to the toxic exposure. Fisher's exact test was used to assess statistical significance. Additional descriptive statistics were also used.

Results: Five hundred and fourteen cases were included. Eighty-six were isolated CCA exposures, 84 were isolated BB exposures, 197 were mixed exposures including a BB, 99 were mixed exposures including a CCA, and 48 were mixed exposures involving both. Toxicological treatment was administered in 357 cases $(69.5 \%)$. The most common treatment was IV fluid resuscitation ( 257 cases, $50 \%$ ), followed by vasopressors (160 cases, $31.1 \%$ ) and glucagon (124 cases, $24.1 \%$ ). Hyperinsulinemiceuglycemic therapy (HIE) was administered in 60 cases $(11.7 \%$ ) and intravenous lipid emulsion (ILE) was used in 25 cases $(4.9 \%$ ). Extracorporeal membrane oxygenation was initiated in 11 cases $(2.1 \%)$. When cases of combined BB and CCA poisoning were excluded, cases involving $\mathrm{BB}$ were more frequently treated with glucagon than cases involving CCA (28.1 vs. $15.1 \%, p=0.0011$ ), while cases involving CCA were more frequently treated with HIE (20.5 vs. $4.6 \%$, $p<0.00001)$, ILE (9.2 vs. $1.8 \%, p=0.0005)$, and/or vasopressors $(35.1$ vs. $24.2 \%, p=0.0119$ ).

Conclusion: BB and CCA poisonings are commonly treated with fluid resuscitation and vasopressors. HIE and ILE are used infrequently. BB poisoning is more frequently treated with glucagon, and CCA poisoning is more frequently treated with HIE, ILE, and/or vasopressors.

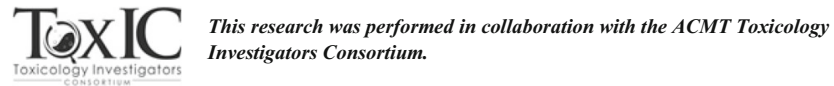

026. Successful Use of Extracorporeal Membrane Oxygenation for Recalcitrant Shock Following Lipid Emulsion Therapy for Polysubstance Ingestion

Robert S Avera ${ }^{1,2}$, Blake Froberg ${ }^{2}$

${ }^{I}$ Indiana University Department of Emergency Medicine, Indianapolis, IN, USA. ${ }^{2}$ Indiana Poison Center, Indianapolis, IN, USA

Background: Extracorporeal membrane oxygenation (ECMO) and lipid emulsion have been described as salvage therapy for recalcitrant cardiogenic shock secondary to xenobiotic ingestion. There has been controversy over the use of these modalities in the same patient, citing concerns that the ECMO equipment may malfunction following lipid emulsion therapy.

Hypothesis: ECMO remains a viable resuscitation modality following lipid emulsion use.

Methods: This is a single patient chart review. A 61-year-old male presented to an outside hospital following an intentional ingestion of ethanol, diltiazem, sotalol, clonidine, sildenafil, gabapentin, and allopurinol. He was hypotensive and bradycardic at presentation. He required high-dose euglycemic insulin therapy, vasopressor support with epinephrine, norepinephrine, and dopamine and still remained bradycardic and hypotensive. Intravenous lipid emulsion therapy with a bolus of $1.5 \mathrm{~mL} / \mathrm{kg}$ $(300 \mathrm{mg} / \mathrm{kg})$ and transvenous pacing were attempted without improvement in vital signs. Six hours following lipid emulsion bolus, venoarterial ECMO (VA-ECMO) was started. ECMO support was continued for a total of 5 days following initial cannulation. 
Results: VA-ECMO was well tolerated and he was weaned off his vasopressor support on hospital day 3. VA-ECMO support was discontinued on hospital day 5 and he was extubated on hospital day 15 . He was neurologically intact and was discharged to inpatient psychiatry on hospital day 17. To our knowledge, no compromise of the ECMO circuit occurred during our patient's care.

Conclusion: We report the successful use of VA-ECMO following ILE. Currently, there are no guidelines to guide the use of both ILE and ECMO concurrently. Our case differs from previous reports of oxygenation membrane and filter compromise following administration of lipid emulsion. This highlights the need to further investigate the relationship between lipid emulsion and ECMO function in critically ill patients.

\section{Tegretol ${ }^{\circledR}$ Trouble: Characterization of Carbamazepine Overdoses in Texas}

Joshua McFalls, Mary Billington, Daniel Tauber, Brett Roth UT Southwestern, Dallas, TX, USA

Background: Carbamazepine (Tegretol@) is a commonly prescribed antiepileptic medication, also utilized in the management of mood disorder and trigeminal neuralgia. In overdose, CNS depression is common; anticholinergic syndrome is possible in large overdose. Carbamazepine demonstrates variable absorption kinetics, with peak therapeutic absorption of 6-24 hours. The active metabolite, a 10,11-epoxide, has equal efficacy with a lengthy half-life of 5-26 hours in chronic users. An anecdotally noted predominance of overdose presentations from detention facilities called to our North Texas Poison Control Center incited an intrigue as to the factual characteristics of carbamazepine ingestions.

Research Question: What proportion of carbamazepine overdoses called to Texas Poison Centers occur in detention centers?

Methods: The Texas Poison Center Network database was queried for all 2019 year to date reports of carbamazepine exposure. Inclusion criteria were defined as age $>18$ years and ingestions which included carbamazepine as an exposure. Multiple ingestion overdoses were not excluded. The resulting pool of cases was then searched by scanning free text for "inmate, prison, or prisoner." Data were confirmed by manual review of cases.

Results: A total of 638 Texas cases were identified. A majority of exposures, $373(58.4 \%)$, involved patients from detention centers. Of this population, approximately one third $(125 / 373 ; 33.5 \%)$ were admitted to a healthcare facility including 57 ICU admissions. The majority of these ICU admissions consisted of single substance carbamazepine overdoses (41 of $57 ; 71.9 \%$ ).

Conclusion: A clear majority of carbamazepine exposures thus far reported to Texas poison centers in 2019 represented patients from detention centers. Of these, one third required hospital admission, nearly half of which required intensive care. These thought-provoking data call attention to unfortunate, preventable, toxic, and costly exposures, and to the need for increased awareness and medication safety measures.

\section{Clearance of Valproate by Hemodialysis in Massive Overdose}

Samuel R York, Alexander M Sidlak, Andrew F Micciche, Rachael C Westover, Shirley Shao, Thomas D Nolin, Anthony F Pizon School of Medicine, University of Pittsburgh, Pittsburgh, PA, USA

Background: Valproate is a small molecule with a low volume of distribution $(0.22 \mathrm{~L} / \mathrm{kg})$, but is highly protein bound at therapeutic doses and therefore is poorly cleared by intermittent hemodialysis (HD). However, in overdose, protein binding becomes saturated and dialysis has been shown to be effective in case reports.

Hypothesis: Hemodialysis demonstrates a high clearance of valproate in massive overdose.

Methods: This is a single patient case report. Serum valproate levels were obtained prior to and after dialysis. Additional serum samples were obtained pre- and post-dialysis cartridge during treatment in order to estimate HD clearance.

Results: A 29-year-old man presented after valproate overdose. He was initially responsive, but developed delirium followed by coma requiring intubation. Valproate level peaked at $2040 \mathrm{mg} / \mathrm{L}, 10$ hours after ingestion. Computed tomography showed no signs of cerebral edema. Ammonia peaked at $355 \mu \mathrm{Mol} / \mathrm{L}$. Due to severity of illness, the patient underwent a single 4.25 hours HD session (Rexeed ${ }^{\mathrm{TM}}-25 \mathrm{SX}$ high flux dialyzer, $450 \mathrm{~mL} /$ minute blood flow) 13 hours post-ingestion. Valproate level 1.5 hours into HD was $739 \mathrm{mg} / \mathrm{L}$ pre-cartridge and $296 \mathrm{mg} / \mathrm{L}$ post-cartridge. HD clearance was calculated to be $163 \mathrm{~mL} /$ minute (higher than the reported range in a systemic review; $51.4-140 \mathrm{~mL} /$ hour). Valproate level declined from $1830 \mathrm{mg} / \mathrm{L}$ to $223 \mu \mathrm{g} / \mathrm{mL}$ during dialysis, indicating an $88 \%$ lowering of serum levels and a half-life of 74.4 minutes. No rebound occurred. Patient improved and was extubated on day 2.

Conclusion: In this case of massive overdose with significantly elevated serum levels ( $>2000 \mathrm{mg} / \mathrm{L}$ ), clearance of valproate with high-flux hemodialysis is higher than previously documented. Early initiation of HD is an effective means to rapidly clear valproate in massive overdose.

\section{Prolonged and Severe CNS Depression and Truncal Ataxia in an Accidental Pediatric Perampanel Ingestion}

Diana J Dean ${ }^{1,2}$, Andrew Schoenling ${ }^{3}$

${ }^{1}$ Michigan Poison Center at Wayne State University, Detroit, MI, USA.

${ }^{2}$ Henry Ford Hospital, Detroit, MI, USA. ${ }^{3}$ University of Pittsburgh Medical Center, Pittsburgh, PA, USA

Background: Perampanel exerts antiepileptic effects by reducing neuronal excitation via noncompetitive antagonism of the postsynaptic ionotropic-AMPA-glutamate receptors. Clinical effects in overdose are limited and only mild effects have been reported in adults.

Methods: This is a single patient chart review.

Results: A previously healthy 2 -year-old $15-\mathrm{kg}$ female presented after witnessed ingestion of $30 \mathrm{mg}$ of perampanel $(2 \mathrm{mg} / \mathrm{kg})$. Within 10 minutes of ingestion, the child became ataxic and was unable to walk. She presented to the ED, 30 minutes post-ingestion and had normal VS but was minimally responsive. She was emergently intubated due to profound CNS depression. After transfer to a tertiary care facility, vitals were as follows: $36.5^{\circ} \mathrm{C}$, HR 117 , BP $113 / 73$, RR 20 (vent), and $100 \%$ on $40 \%$ $\mathrm{FiO}_{2}$. She required no sedation and ABG showed 7.24/56/65. Serum laboratory results were otherwise unremarkable. UDS immunoassay and GC-MS were both negative. She remained hemodynamically stable and remained intubated for 72 hours with gradual improvement in mental status. After extubation, patient still exhibited truncal ataxia and did not return to her neurologic baseline until 96 hours post-ingestion. Serum perampanel level was $870 \mathrm{ng} / \mathrm{mL}$ ( $\mathrm{ref}<20 \mathrm{ng} / \mathrm{mL}$ ).

Discussion: To date, there are no reported pediatric ingestions of perampanel. Adult toxicity describes mild neurologic symptoms. In this case, our patient exhibited profound CNS depression requiring mechanical ventilation for a prolonged period. At this time, we suggest all pediatric exposures be referred to the ED.

Conclusion: This is the first reported confirmed case of perampanel overdose in a child. Pediatric toxicity may result in profound CNS depression requiring prolonged mechanical ventilatory support.

030. Characteristics of Pediatric Lead Admissions Seen by Medical Toxicology Service

Diana J Dean ${ }^{1,2}$, Elizabeth Jacobs ${ }^{1,3}$, Andrew M King ${ }^{1,3}$, Eric Malone ${ }^{2,1}$, Cynthia K Aaron ${ }^{1}$

${ }^{I}$ Michigan Poison Center at Wayne State University, Detroit, MI, USA.

${ }^{2}$ Henry Ford Hospital, Detroit, MI, USA. ${ }^{3}$ Detroit Medical Center, Detroit, MI, USA 
Background: Pediatric lead poisoning is a frequent consultation to our toxicology service. In partnership with pediatric hematology, the toxicology service developed a unique protocol that includes admission and chelation for patients with blood lead levels (BLL) $>40 \mathrm{mcg} / \mathrm{dL}$. Chelation is performed with DMSA and IV CaNa2EDTA infusion for 120 hours. Admission removes the child from further exposure and allows the health department to identify and mitigate the source. Further, admission facilitates family education and nutritional counseling, arranges close follow-up with our lead clinic, and provides expeditious access to ancillary services to optimize health outcomes.

Methods: This is a retrospective review of pediatric medical toxicology consultations with the diagnosis of lead intoxication, plumbism, and lead exposures. Results: Of the 101 consultations completed between July 2013 and August 10, 2019, there were 77 unique patients with 24 (23.8\%) having $>2$ hospitalizations and 4 with $>3$ hospitalizations. The average LOS was 6 days and average presenting BLL was $41.5 \mathrm{mcg} / \mathrm{dL} ; 38 \%$ of patients had radiopaque foreign bodies presumed to be lead and were administered whole bowel irrigation; 74/101 consultations were chelated. There were infrequent minor reported adverse effects and no moderate or major effects of chelation.

Discussion: Compliance with less-aggressive and prolonged outpatient succimer protocols can be problematic due to availability of succimer and lack of knowledgeable providers. Further, a large proportion requires acute gut decontamination. This protocol is intensive and comprehensive. A subset of patients had repeat admissions for persistently elevated BLL likely from re-exposure or redistribution.

Conclusion: We find that hospitalization at a specialty center, utilizing this protocol, efficiently coordinates medical and governmental social support services: it is safe and comprehensive.

031. The Chogosta of Queens, NY: Neonatal Lead Poisoning After Cultural Consumption of Contaminated Soil

Rachel M Shively

Northwell Health, Manhasset, NY, USA. Elmhurst Hospital - Mount Sinai Health System, New York, NY, USA

Background: Pica is particularly common in pregnant women, and while micronutrient deficiencies might spur the craving in some, it may also be prompted by culinary traditions. The "chogosta" is one such ancestral food, a clay ball traditionally eaten in the Mexican municipality of Jaltipan. When residents carry these practices to new environments with contaminated soils, they risk poisoning, often by heavy metals like lead.

Methods: We report a case of maternal and neonatal lead poisoning, after the mother ate chogosta, made from local Queens, NY soil during her pregnancy. Results: A 35-year-old Mexican woman was found to have an elevated lead level after prenatal screening identified an unexplained microcytic anemia. At 12 and 25 weeks gestation, her serum lead level was 7 and $2 \mu \mathrm{g} / \mathrm{dl}$, respectively. Although she was consuming homemade chogosta in Queens, NY, this practice was not disclosed to her doctors and the behavior continued. Upon uncomplicated delivery of a baby girl, the mother's lead level was $47 \mu \mathrm{g} / \mathrm{dl}$. Four days later, mother and baby serum lead levels were 51 and $65 \mu \mathrm{g} / \mathrm{dl}$, respectively. Breast-feeding was stopped and succimer $350 \mathrm{mg} / \mathrm{m}^{2}$ TID was initiated. Baby girl's lead level at 10 days old was $40 \mu \mathrm{g} / \mathrm{dl}$, but she completed a course of succimer $350 \mathrm{mg} / \mathrm{m}^{2}$ TID for 5 days and BID for 14 days. After the 19-day course, her lead level was $12 \mu \mathrm{g} / \mathrm{dl}$. The mother was also treated with succimer, but aborted it once a pre-succimer level returned at $39 \mu \mathrm{g} / \mathrm{dl}$. Neither patient exhibited lead toxicity. The mother disclosed the practice of consuming chogosta after succimer initiation. Her medical team provided education about pica risks.

Conclusion: Pica of contaminated soils can lead to lead toxicity. At-risk patients should be questioned about cultural practices that may lead to this exposure
032. Acute Deterioration of a Pediatric Patient After Ingestion of Liquid Formulation Iron Sulfate

Richard J Chen, Muhammed Ershad, James Krueger Einstein Healthcare Network, Philadelphia, PA, USA

Background: Toxicity following the ingestion of liquid formulations of elemental iron is not well described in the literature. We report a case of pediatric ingestion of a liquid iron formulation with early presentation of significant toxicity.

Methods: This is a single patient chart review. A 2-year-old female presented to the emergency department 1 hour after accidental ingestion of a liquid formulation of iron sulfate. The patient was estimated to have ingested a total of $750 \mathrm{mg}$ of elemental iron and had multiple episodes of emesis prior to arrival. She was somnolent and difficult to arouse requiring emergent intubation. Nasogastric aspiration of the ingested contents was attempted. Deferoxamine infusion was empirically started 2 hours post-ingestion, before initial iron levels were available as patient clinical status had worsened. Infusion rate was started at $5 \mathrm{mg} / \mathrm{kg} /$ hour and titrated to $15 \mathrm{mg} / \mathrm{kg} /$ hour.

Results: Serum iron levels were obtained 1 hour post-ingestion and 2 and 4 hours after starting deferoxamine infusion. Her serum iron concentrations were 314,269 , and $223 \mathrm{mcg} / \mathrm{dL}$. The patient experienced mild hypotension that was fluid responsive. She was maintained on deferoxamine for 24 hours with eventual resolution of her metabolic acidosis and continued downtrend in iron levels. The patient did not develop vin rosé coloration of her urine. Additional iron levels obtained during the patient's hospital course were 146 and $50 \mathrm{mcg} / \mathrm{dL}$. The patient was extubated and discharged 2 days after initial presentation with full recovery.

Conclusion: Liquid formulations of iron may be associated with earlier and clinically more severe toxicity profiles than the conventional tablet formulations, warranting earlier administration of deferoxamine.

\section{Pediatric Respiratory Failure Requiring Prolonged ECMO from Household Inhalational Exposure to Elemental Mercury}

William J Meggs ${ }^{1}$, Tiffany Byerly ${ }^{1}$, Keegan Ziemba ${ }^{1}$, Kerry Shum ${ }^{1}$, Mark Dexter ${ }^{1}$, Matthew Ledoux ${ }^{1}$, Ricky Langley ${ }^{2}$, Anna R Dulaney ${ }^{3}$, Michael Beuhler ${ }^{3}$

${ }^{1}$ Brody School of Medicine at East Carolina University, Greenville, NC, USA. ${ }^{2}$ North Carolina Department of Health and Human Services, Raleigh, NC, USA. ${ }^{3}$ Carolinas Poison Center, Charlotte, NC, USA

Background: Inhalational exposure to elemental mercury can cause pneumonitis. A 19-month-old girl had respiratory failure from elementary mercury exposure in her home.

Methods: This is a case report.

Results: A 19-month-old girl presented with acute respiratory distress, cough, fever, and hypoxia after traveling from Guatemala to join her father, a field worker. Her 4-year-old brother had a milder presentation. Room air $\mathrm{O} 2$ saturation was $65 \%$, pulse 180 beats/minute, respiratory rate 40 breaths/minute, afebrile. Chest X-ray demonstrated pulmonary opacities. She was transferred to a pediatric intensive care unit. Family denied chemical exposures. Progressive respiratory failure required intubation on hospital day 2 and ECMO on day 4. No infection was found. Toxicology differential included respiratory irritants such as paraquat and elemental mercury. Blood mercury level was $145 \mathrm{mcg} / \mathrm{L}$. Spot urine mercury was $1725 \mathrm{mcg} / \mathrm{g}$ creatinine. Chelation was begun with succimer. Residents were removed from the home. She was transferred to rehabilitation on hospital day 52 with multiple co-morbidities. Parents and a family sharing their mobile home surrounded by farm fields were asymptomatic but were found to have elevated mercury levels. Her mother was 6 weeks pregnant, had a mercury level of $313 \mathrm{mcg} / \mathrm{L}$, refused chelation, and was lost to follow-up. Her brother was discharged on hospital day 2 with a normal examination and was subsequently found to have a level of $231 \mathrm{mcg} / \mathrm{L}$. An 
asymptomatic 2.5 -year-old girl had a level of $131 \mathrm{mcg} / \mathrm{L}$, was started on succimer, as were her parents. The home air had elevated mercury levels, with a level of $1500 \mathrm{ng} / \mathrm{m}^{3}$ in the sink drain. No source was found. The owner of the home reported a cluttered interior that was cleaned prior to inspection. A methamphetamine laboratory was suspected, but law enforcement could not find evidence.

Conclusion: Consideration of elemental mercury exposure as a cause of respiratory failure in children should be considered even if parents deny home exposures.

\section{An Atypical Caustic Ingestion-Zinc Chloride with Hyperzincemia}

Robert C Pueringer, Jon B Cole, Annie M Arens Minnesota Poison Control Center, Minneapolis, MN, USA

Background: Zinc chloride ingestions are infrequent and typically lead to corrosive gastrointestinal injury and rarely lethargy or shock. We present an unintentional acute zinc chloride ingestion with hyperzincemia (high serum zinc)

Methods: This is a case report of a 50-year-old male presenting to the ED within hours of accidental occupational zinc chloride exposure and ingestion. He was sprayed/splashed in the face and swallowed a "few" mouthfuls of the fertilizer additive zinc chloride after a pressurized hose broke. Within 60 minutes, he developed abdominal pain, nausea, repeated nonbloody emesis, and diaphoresis. Initial vital signs were as follows: blood pressure $119 / 71 \mathrm{mmHg}$, pulse $98 \mathrm{bpm}$, temperature $36^{\circ} \mathrm{C}$, respiratory rate 16 , and oxygen saturation $98 \%$ on room air. Examination was significant for mild epigastric tenderness. Esophagogastroduodenoscopy revealed grade I caustic esophagitis and gastritis. CT imaging confirmed no perforations. Laboratory studies, including serum electrolytes and copper levels, were normal barring a zinc concentration of $188 \mathrm{mcg} / \mathrm{dL}$ (normal $80-120$ ) that returned several days later. He was not treated with chelation therapy. Ultimately, symptoms improved within days and had not recurred by 4 months.

Results: Zinc chloride ingestions are rare and literature is scant. Typical ingestions cause corrosive GI injury, and severe ingestions cause nausea/ vomiting, lethargy, electrolyte aberrations (hypocalcemia, hypocupremia, hyperzincemia), and potentially cardiovascular collapse. Pancreatitis and pulmonary edema or pneumonitis have been documented. Treatment is supportive. There are no clear indications for chelation therapy, either prophylactically or empirically for hyperzincemia. Chronic hyperzincemia can cause hypocupremia (leading to cytopenias), pancreatic insufficiency, or memory/learning deficits. We planned chelation with EDTA if the patient developed hemodynamic instability or signs of end-organ toxicity.

Conclusion: Zinc chloride ingestions typically cause corrosive gastrointestinal injury and hyperzincemia. There are no clear indications for chelation. We present a patient with hyperzincemia after a small accidental zinc chloride exposure who did not require empiric chelation.

\section{Aluminum Encephalopathy May not Correlate to Aluminum Level but Still Corrects with Chelation Therapy}

Wells J Brambl, Vincent R Lee, Joshua N Nogar

Northwell Health, Manhasset, NY, USA

Background: Aluminum toxicity is associated with aluminum sulfate bladder irrigation products as well as dialysate for dialysis patients. The clinical features of acute aluminum toxicity involve acute onset encephalopathy after exposure, typically within several hours.

Hypothesis: We hypothesize that aluminum-associated encephalopathy does not always correlate with the magnitude of the serum aluminum level.

Methods: We present our case, clinical course, and review the literature.
Results: A 90-year-old male with past medical history of CAD s/p CABG, bladder CA status post-radiation cystitis was admitted for hematuria and continuous bladder irrigation. He then underwent open repair for an extraperitoneal bladder perforation. Over the next 5 days, he received $9 \mathrm{~L}$ of $1 \%$ aluminum sulfate bladder irrigation. He became acutely altered with slurred speech and disorientation on irrigation day \#5, which progressed to obtundation 12 hours later. CT head, blood/urine cultures were all unremarkable. An aluminum level from that morning was $18 \mathrm{mcg} / \mathrm{L}$. He was started on olanzapine for "agitation." He underwent bilateral percutaneous nephrostomy drains to bypass gross hematuria. The next day, a trial of chelation therapy with deferroxamine (DFO) was given $(15 \mathrm{mg} / \mathrm{kg}$ ) over 2 hours. After a second dose of DFO, his level fell to $8 \mathrm{mcg} / \mathrm{L}$. His mental status began to improve the next day. Approximately 72 hours after DFO therapy, his mental status returned to baseline.

Conclusion: The magnitude of serum aluminum levels may not necessarily indicate the degree of encephalopathy present. Encephalopathy associated with aluminum exposure may occur at levels barely higher than normal range.

\section{QRS Widening Associated with Cyclobenzaprine Overdose}

Wells J Brambl, Vincent R Lee, Joshua N Nogar

Northwell Health, Manhasset, NY, USA

Background: Cyclobenzaprine bears a structural resemblance to tricyclic antidepressants (TCA). However, to the best of our knowledge, there have been no reports of cyclobenzaprine-induced sodium channel blockade manifesting as prolonged QRS on ECG.

Hypothesis: Cyclobenzaprine may be associated with a widened QRS.

Methods: We present our case, clinical course, and review the literature. Results: A 68-year-old patient with past medical history of hypertension, chronic pain, and depression presented to the ED with an intentional ingestion of an unknown amount of cyclobenzaprine and EtOH. Initial vital signs were as follows: $\mathrm{BP}, 65 / 42 \mathrm{mmHg}$;, 65 ; RR, 15; O2sat, $85 \%$ on room air; $\mathrm{T}, 98.3^{\circ} \mathrm{F}$. Initial EKG showed a wide complex sinus rhythm with a QRS of $160 \mathrm{~ms}$. Routine laboratory studies were significant for a BUN/Cr of 23/1.81, pH 7.13, AST 114 IU/L, ALT 57 IU/L, and EtOH of $169 \mathrm{mg} / \mathrm{dL}$. His EKG normalized to a QRS of $80 \mathrm{~ms}$ the next day, by which time he was more lucid, and his vital signs had normalized. A cyclobenzaprine level was drawn from the initial set of specimens, which resulted in a cyclobenzaprine level of $122 \mathrm{ng} / \mathrm{mL}$ (therapeutic range $=$ $10-30 \mathrm{ng} / \mathrm{mL}$ ). A serum screen for TCAs was negative.

Conclusion: Large cyclobenzaprine levels may be associated with prolongation of the QRS interval, in a fashion similar to TCAs. Limitations to this study are that it is a retrospective case report and the patient was also initially acidemic due to a primary respiratory acidosis, which can also prolong the QRS interval.

\section{Abstract Withdrawn}

\section{Glyphosate Exposures Reported to Poison Centers}

Shawn M Varney ${ }^{1,2}$, Mathias B Forrester ${ }^{3}$

${ }^{1}$ University of Texas Health - San Antonio, San Antonio, TX, USA. ${ }^{2}$ South Texas Poison Center, San Antonio, TX, USA. ${ }^{3}$ Austin, TX, USA

Background: Glyphosate is an organophosphorus compound in hundreds of herbicide products and has low toxicity for humans. Glyphosate exposure can result in abdominal cramps, diarrhea, dizziness, headache, eye/mouth/throat/skin irritation, difficulty breathing, and hypotension, depending on the exposure route. There is limited information on human exposures to glyphosate. Our objective was to describe glyphosate exposures reported to a poison center network.

Methods: Cases were glyphosate exposures reported to a statewide poison center network during 2000-2018. Case distribution was determined 
for factors related to patient demographics, exposure circumstances, management, and outcome.

Results: We identified 2335 glyphosate exposures. Distribution by patient age was $654(28.0 \%) 0-5$ years, $114(4.9 \%) 6-12$ years, 92 (3.9\%) $13-19$ years, and $1444(61.8 \%) 20$ years or older; $816(34.9 \%)$ were female. Most common routes were 1075 (46.0\%) dermal, 1050 (45.0\%) ingestion, $383(16.4 \%)$ ocular, and 301 (12.9\%) inhalation. Exposure reason was $2198(94.1 \%)$ unintentional, $82(3.5 \%)$ intentional, and 29 (1.2\%) adverse reaction. Unintentional misuse accounted for $583(25.0 \%)$ exposures. April-July accounted for 1271 (54.4\%) exposures. Medical outcomes were $413(17.7 \%)$ no effect, 405 (17.3\%) minor effect, 86 $(3.7 \%)$ moderate effect, $8(0.3 \%)$ major effect, $101(4.3 \%)$ not followed - judged nontoxic, 1025 (43.9\%) not followed - minimal clinical effects possible, 85 (3.6\%) unable to follow-potentially toxic, and $207(8.9 \%)$ unrelated effect. Five $(0.2 \%)$ deaths were reported. Most frequent clinical effects were ocular irritation/pain $(n=238,10.2 \%)$, vomiting ( $n=190,8.1 \%)$, nausea $(n=158,6.8 \%)$, dermal irritation/pain $(n=156,6.7 \%)$, red eye $(n=93,4.0 \%)$, erythema/flushed skin $(n=90$, $3.9 \%)$, headache $(n=74,3.2 \%)$, and throat irritation $(n=73,3.1 \%)$. Most frequent treatments were dilute/irrigate/wash $(n=1815,77.7 \%)$, food/ snack $(n=119,5.1 \%)$, and fresh air $(n=110,4.7 \%)$.

Conclusion: Most glyphosate exposures involved adults and males, dermal contact or ingestion, were unintentional, and did not result in serious outcomes. Five deaths occurred.

\section{Capsaicin Medication Exposures Reported to Poison Centers}

Shawn M Varney ${ }^{1,2}$, Mathias B Forrester ${ }^{3}$

${ }^{1}$ University of Texas Health - San Antonio, San Antonio, TX, USA. ${ }^{2}$ South Texas Poison Center, San Antonio, TX, USA. ${ }^{3}$ Austin, TX, USA

Background: Capsaicin is a topical medication with anti-inflammatory and analgesic properties used to manage osteoarthritis, neuropathy, and cannabinoid hyperemesis syndrome. Capsaicin is a powerful irritant and can cause dermal burning or stinging. If ingested, capsaicin can result in nausea, vomiting, abdominal pain, and diarrhea. Ocular exposure can cause tearing, ocular pain, and conjunctivitis. Our objective was to describe capsaicin medication exposures reported to poison centers.

Methods: Cases were single-agent capsaicin exposures reported to a statewide poison center network during 2000-2018. Pepper spray exposures were excluded. We determined case distribution for factors related to patient demographics, exposure circumstances, management, and outcome.

Results: We identified 1376 capsaicin exposures. Exposures declined from 129 in 2000 to 29 in 2018. Patient age distribution was 435 (31.6\%) 0-5 years, $65(4.7 \%) 6-12$ years, 58 (4.2\%) 13-19 years, 490 (35.6\%) $20-59$ years, and $154(11.2 \%) 60$ years or older; $800(58.1 \%)$ were female. Most common routes were 716 (52.0\%) dermal, 540 $(39.2 \%)$ ingestion, and $211(15.3 \%)$ ocular. Exposure reason was 1035 $(75.2 \%)$ unintentional, $332(24.1 \%)$ adverse reaction, and $7(0.5 \%)$ intentional. Medical outcomes were $64(4.7 \%)$ no effect, 454 (33.0\%) minor effect, $40(2.9 \%)$ moderate effect, $1(0.1 \%)$ major effect, $36(2.6 \%)$ not followed - judged nontoxic, $764(55.5 \%)$ not followed - minimal clinical effects possible, $14(1.0 \%)$ unable to follow-potentially toxic, and 3 $(0.2 \%)$ unrelated effect. No deaths were reported. The most frequent clinical effects were dermal irritation/pain $(n=678,49.3 \%)$, erythema/ flushed ( $n=276,20.1 \%)$, ocular irritation/pain $(n=199,14.5 \%)$, oral irritation $(n=196,14.2 \%)$, red eye $(n=64,4.7 \%)$, and superficial dermal burns $(n=44,3.2 \%)$. Most frequent treatments were dilute/irrigate/wash $(n=1258,91.4 \%)$, food/snack $(n=65,4.7 \%)$, and antihistamines $(n=17$, $1.2 \%)$.

Conclusion: The majority of capsaicin medication exposures occurred by dermal, ingestion, or ocular routes and were unintentional or adverse reactions. Most exposures did not result in serious outcomes.

\section{Death from Presumed Tamsulosin Toxicity}

Matthew D Eisenstat, Lindsay Schaak, Michael Yeh, Colin Therriault, Alaina Steck

Emory University, Atlanta, GA, USA

Background: Tamsulosin is an alpha (1A) receptor blocker that is commonly prescribed for the treatment of benign prostatic hyperplasia. We report the first case in literature of a death thought to be predominantly caused by a tamsulosin overdose.

Methods: This is a single patient case report. The patient is a 65 -year-old male with a past medical history of HIV, hepatitis C, schizophrenia, and cocaine use disorder who presented to the emergency department (ED) after ingestion of three bottles of his home medications in a suicide attempt. Per report from EMS, the three bottles were 30-day supplies of tamsulosin $0.4 \mathrm{mg}$ (30 tablets), Genvoya ${ }^{\circledR}$ (elvitegravir, cobicistat, emtricitabine, tenofovir) 150-150-200-10 mg (30 tablets) and Prezista ${ }^{\circledR}$ (darunavir) $800 \mathrm{mg}$ (30 tablets). The patient arrived without complaints and with stable vitals (HR $93 \mathrm{bpm}$, BP 147/78 mmHg, RR 20/ minute, temp $97.3^{\circ} \mathrm{F}$ ). Initial results of $\mathrm{CBC}$, CMP, lipase, salicylate, APAP, EtOH, VBG, and chest X-ray were unremarkable. EKG showed RBBB morphology (new) with QRS 137 ms, QTc 496 ms.

Results: After approximately 10 hours of uneventful observation, the patient developed a precipitous drop in blood pressure to $60 / 40 \mathrm{mmHg}$ with a concurrent decrease in mental status. He was started on norepinephrine after his blood pressure failed to respond to $2 \mathrm{~L}$ of intravenous crystalloid boluses. Toxicology recommended phenylephrine as a second vasopressor in an attempt to counteract the known mechanism of action of tamsulosin. The patient was admitted to the ICU and had continued decline of clinical status and increasing vasopressor requirements throughout the day. Despite the use of multiple vasopressors (norepinephrine, phenylephrine, vasopressin; later changed to norepinephrine, phenylephrine, and angiotensin II), the patient developed PEA arrest and was subsequently pronounced dead. No alternate etiology was discovered during ICU evaluation. Medical examiner's report is pending.

Conclusion: This case is the first to our knowledge of an overdose of tamsulosin leading to mortality. While other factors may have contributed to this patient's outcome, this case challenges the dogma that tamsulosin is benign in overdose.

\section{Asymptomatic Escitalopram and Citalopram Overdoses: 6- Hour Observation Period?}

Vincent R Lee ${ }^{1}$, Mark T Huang ${ }^{1}$, Wells J Brambl ${ }^{1}$, Stephanie Widmer ${ }^{1}$, Benjamin D Kessler ${ }^{2}$, Nima Majlesi ${ }^{2}$, Joshua N Nogar ${ }^{1}$, Daria Falkowitz ${ }^{1}$ ${ }^{I}$ Northwell Health, New Hyde Park, NY, USA. ${ }^{2}$ Staten Island University Hospital, Staten Island, NY, USA

Background: Supratherapeutic escitalopram and citalopram ingestions have been associated with delayed onset of QT prolongation with some authorities recommending a minimum observation period of 24 hours.

Hypothesis: Asymptomatic patients presenting with a supratherapeutic ingestion of escitalopram or citalopram with an initial QT-corrected (QTc) interval of less than $500 \mathrm{~ms}$ do not experience significant delayed QT prolongation.

Methods: This is a retrospective case series from the Northwell Health Hospital system. We identified cases between 7 November 2017 and 10 November 2019 by searching (terms: escitalopram, citalopram) from our consultant database. We included cases of reported supratherapeutic single-agent ingestions. We excluded reported co-ingestions of known QT-prolonging xenobiotics, missing EKG-interval values, and/or missing electrolyte values.

Results: We identified $n=13$ cases with a reported supratherapeutic ingestion of escitalopram or citalopram. We excluded four cases due to coingestants of QT-prolonging drugs, leaving nine single-agent overdose cases (mean age $=19$ years (range $1.5-40$ years), female patients $(n=8)$, 
intentional $(n=7)$, unintentional/exploratory $(n=2)$, escitalopram $(n=8)$, citalopram $(n=1))$. The mean time from reported ingestion to initial EKG was 154 minutes. In escitalopram cases, the mean reported dose ingested was $132 \mathrm{mg}$ (range 10-440 mg), mean initial and discharge QTc interval were 443 and $441 \mathrm{~ms}$, respectively. In the single citalopram case, the intent was exploratory, dose was $20 \mathrm{mg}$, initial QTc interval was $435 \mathrm{~ms}$, and discharge QTc interval was $409 \mathrm{~ms}$. No patients received GI decontamination. All patients presented asymptomatic with an unremarkable EKG, electrolytes, and urine drug screen. All patients were admitted for cardiac telemetry for 24-hours post-ingestion. There were no cardiac events from initial presentation to discharge.

Conclusion: In this case series, reported overdoses of escitalopram or citalopram presenting with an initial QTc interval less than $500 \mathrm{~ms}$ did not exhibit significant delayed QT-interval prolongation and may not necessarily have required an extended observation period.

\section{Curse of the Ghost Pill: a Case Report and Literature Review}

Vincent R. Lee, Daria Falkowitz, Wells Brambl, Stephanie Widmer, Joshua N. Nogar

Northwell Health, New Hyde Park, NY, USA

Background: Controlled-release (CR) medications are often formulated with insoluble shell-housings that are excreted into the stool intact, a phenomena known as "ghost pills." It is unclear if ghost pills found in the rectal effluent during whole-bowel irrigation (WBI) represent unabsorbed active drug or the shell-casing of an already absorbed drug.

Hypothesis: There is a lack of literature evaluating "ghost pills" and their impact on the management for the poisoned patient.

Methods: This is a case report and literature review on "ghost pills." A 17year-old female with a past medical history of depression presented with unresponsiveness and seizures after an overdose. Her initial vital signs were as follows: HR 160, BP 122/66, RR 40, and afebrile. She was intubated for airway protection and arrived to our tertiary center 18 hours after presentation. The ingestion included an unknown amount of $450 \mathrm{mg}$ bupropion XL, $100 \mathrm{mg}$ desvenlafaxine, and $5 \mathrm{mg}$ olanzapine. WBI was recommended at $1 \mathrm{~L} /$ hour with the appearance of multiple "intact pills" in the rectal effluent. Twelve hours later, the rectal effluent was clear and WBI was discontinued. However, intact pills continued to appear in the patient's stool through hospital day 5, prompting the question as to whether WBI should be restarted. Our suspicion was that these were "ghost pills" and WBI was not restarted, given the patient's resolving toxicity.

Results: Current literature largely consists of case reports and emphasizes the lack of awareness of the "ghost pill" phenomenon by both patients and clinicians. Additionally, there are reports on the formation of "ghost pill" bezoars due to the insoluble components. Available CR medications that are known to produce ghost pills include desvenlafaxine, dexmethylphenidate, methylphenidate, venlafaxine, bupropion, oxycodone, oxybutynin, carbamazepine, and paliperidone.

Conclusion: The presence of "ghost pills" after WBI in this patient's overdose was an expected phenomenon. The "ghost pill's" reappearance after discontinuing WBI may have been secondary to the CR medication's tendency to form bezoars.

\section{Oral Baclofen Treatment for Gamma-Hydroxybutyrate Withdrawal}

Vincent R Lee, Daria Falkowitz, Wells J Brambl, Stephanie Widmer, Mark T Huang, Joshua N Nogar

Northwell Health, New Hyde Park, NY, USA

Background: Gamma-hydroxybutyrate (GHB) has potent sedative effects exerted via GABA-B agonism. Cessation of chronic GHB use produces an abrupt and challenging withdrawal syndrome that is notoriously resistant to conventional treatment with GABA-A agonists.

Hypothesis: Mild GHB withdrawal can be effectively treated with initiation of an oral baclofen taper.
Methods: This is a retrospective case report from a large tertiary city hospital. A 28-year-old man with a history of opioid and GHB dependence who presented requesting detox for GHB. He reports the gradual onset of restlessness, generalized tremors, palpitations, agitation, and anxiety over 2 hours. The patient has reported daily consumption for the past 4.5 months of 15 $45 \mathrm{ml}$ of internet-procured GHB to self-treat pain from epididymitis. His last reported GHB dose was 8 hours prior to presentation. His vital signs remained hemodynamically stable with a physical exam significant for upper and lower extremity tremors, intermittent agitation, and anxiety. His GHB level was $0 \mathrm{mg} / \mathrm{ml}$. Despite treatment with $4 \mathrm{mg}$ of IV lorazepam, the patient continued to have worsening symptoms. At this point, we initiated an oral baclofen taper over 5 days with the following protocol: day 1, $20 \mathrm{mg}$ every 6 hours; day 2 , $20 \mathrm{mg}$ every 8 hours; day 3, $20 \mathrm{mg}$ every 12 hours; day 4, $20 \mathrm{mg}$ once; and day 5 , discontinuation of baclofen. The patient was evaluated under the CIWA protocol for supplemental baclofen given as needed every 4 hours.

Results: The patient reported full resolution of his symptoms after the first dose of baclofen on day 1 . Throughout the taper period, there was no relapse of his withdrawal syndrome and he did not require any supplemental PRN doses. The patient left against medical advice on day 4 of treatment.

Conclusion: Introduction of a baclofen taper is a receptor-specific treatment option and successfully resolved this patient's mild GHB withdrawal symptoms.

\section{Candy Coma: a Case Series of Etizolam Exposures}

Jennifer S Love ${ }^{1}$, John A Thompson ${ }^{1}$, Faye Girardi ${ }^{2}$, Michael Letsky ${ }^{2}$, Lauren Murphy ${ }^{1}$, B Zane Horowitz ${ }^{1}$

${ }^{I}$ Oregon Health \& Science University, Portland, OR, USS. ${ }^{2}$ Oregon Poison Center, Portland, OR, USA

Background: Exposures to novel psychoactive substances, including designer benzodiazepines, are increasingly reported to national poison centers.

Methods: This is a case series of three patients using poison center and hospital medical record data. Three children, ages 10 years (patient A), 9 years (patient B), and 6 years (patient $C$ ) old, presented to an emergency department (ED) after ingesting unlabeled "Pez" candy at home. The children were found somnolent, drooling, and ataxic. On arrival to the $\mathrm{ED}$, all three children were drowsy, and patient $\mathrm{C}$ had pinpoint pupils. All children received naloxone without mental status improvement. Patient C's urine drug screen was positive for benzodiazepines. During their ED visit, patients $\mathrm{A}$ and $\mathrm{C}$ became more obtunded and were given flumazenil $0.2 \mathrm{mg}$. Both patients became more alert following flumazenil. Patients A and $\mathrm{C}$ were transferred to the children's hospital ICU for further monitoring. On arrival, patient A had dysarthria and drowsiness and patient $C$ had slurred speech and hallucinations. Both patients' symptoms resolved after 72 hours; they did not receive additional flumazenil in the ICU. Gas chromatography mass spectrometry was performed on patient C's urine. Results revealed a detectable etizolam concentration. Patient B's mental status improved without flumazenil. He was admitted to pediatrics and discharged 24 hours later.

Conclusion: Etizolam is a thienodiazepine, which is a short-acting benzodiazepine derivative. It is prescribed in Japan, Italy, and India as treatment for anxiety and sleep disorders. Etizolam is approved for experimental use in the USA, but within the last 3 years, some states have designated it a controlled substance. Our case highlights a pediatric public health threat associated with etizolam. Etizolam was concealed by being pressed into a "Pez" candy shape. This method of drug concealment is alarming because, as shown here, children will mistake this for candy.

045. Two Cases of Attempted Suicide with Pentobarbital, Ordered Online Through Contacts Found in The Peaceful Pill Handbook

Han T Gao ${ }^{1}$, Heather A Borek ${ }^{2}$, Aaron Schneir ${ }^{1}$ 
${ }^{1}$ University of California San Diego, San Diego, CA, USA. ${ }^{2}$ University of Virginia School of Medicine, Charlottesville, VA, USA

Background: Pentobarbital is a barbiturate that is most commonly used in veterinary medicine. However, in recent years, pentobarbital has gained notoriety as a drug of choice for use in assisted suicides, leading to "suicide tourism" to Mexico where pentobarbital is easily purchased. Hypothesis: With the prevalence and ease of the "dark web," pentobarbital can now be readily ordered online, leading to cases of overdose nationwide.

Methods: This is a chart review of two patients. Case 1-A 16-year-old male in California was found unresponsive at home next to two empty bottles labeled "Natura Skin Cleanser Face Masque" and was intubated due to respiratory depression. The family provided access to his encrypted emails showing he had purchased pentobarbital online. Case 2-A 44-year-old female in Virginia presented with somnolence and slurred speech after drinking an unknown amount of pentobarbital and admitted to purchasing two similarly labeled bottles online off the "dark web" as a suicide attempt.

Results: In case 1, the patient had an initial pentobarbital serum concentration of $85 \mathrm{mcg} / \mathrm{mL}$ (therapeutic $1-5 \mathrm{mcg} / \mathrm{mL}$ ) and was intubated for 6 days. Though he developed an aspiration pneumonia and a catheterassociated urinary tract infection, he made a full neurological recovery. In case 2, the patient had an uncomplicated course with improvement the following day. She had a urinary pentobarbital concentration of $2347 \mathrm{ng} /$ $\mathrm{mL}$ (reporting limit $>200 \mathrm{ng} / \mathrm{mL}$ ) and the bottles tested positive for pentobarbital. In both cases, the patients admitted to ordering bottles that were deceptively labeled from a veterinarian in Mexico. His email was listed in a modified e-book version of "The Peaceful Pill Handbook," which detailed various methods of purchasing pentobarbital.

Conclusion: Clinicians should be aware that pentobarbital can be readily ordered online as a means for suicide and may be disguised as a seemingly innocuous herbal product.

\section{Refractory Vasoplegic Shock After Sodium Azide Ingestion} Treated with Hemodialysis

Han T Gao, Samantha EM McGlone, Binh T Ly

University of California San Diego, San Diego, CA, USA

Background: Sodium azide $\left(\mathrm{NaN}_{3}\right)$ is a white crystalline solid commonly used as a laboratory preservative and an airbag propellant and in explosive manufacturing. In toxicity, $\mathrm{NaN}_{3}$ is a potent vasodilator and inhibits mitochondrial cytochromes resulting in cellular asphyxiation and cardiovascular collapse. It is not previously known if $\mathrm{NaN}_{3}$ is removed by hemodialysis.

Hypothesis: Hemodialysis may be considered for $\mathrm{NaN}_{3}$ ingestions presenting with vasoplegia and shock refractory to standard supportive care. Methods: This is a single patient case report. A 23-year-old male laboratory technician ingested $1.38 \mathrm{~g}$ of $\mathrm{NaN}_{3}$. Immediately following the ingestion, he began to feel flushed, diaphoretic, and nauseous, followed by one episode of emesis. His roommate called 911 and he arrived at the emergency department with these initial vital signs: HR $120 \mathrm{bpm}$ and BP $92 / 40 \mathrm{mmHg}$. He was given a total of $4 \mathrm{~L}$ of intravenous crystalloid without improvement in BP. Two hours after his ingestion, he began to complain of severe chest pain. A norepinephrine infusion was started at $2.5 \mathrm{mcg} /$ minute and quickly titrated up to $10.5 \mathrm{mcg} / \mathrm{minute}$ without improvement in symptoms or BP. The decision was then made to initiate hemodialysis given the low molecular weight of $\mathrm{NaN}_{3}$ at $65 \mathrm{Da}$.

Results: Serum lactate was $5.3 \mathrm{mmol} / \mathrm{L}$ (reference $0.5-2.0 \mathrm{mmol} / \mathrm{L}$ ). Bedside echocardiogram demonstrated normal cardiac output. A 6-hour hemodialysis session was initiated 5 hours post-ingestion with worsening of his vasoplegia and hypotension at BP $89 / 48 \mathrm{mmHg}$. Vasopressin infusion was added during hemodialysis and his norepinephrine dose was increased to $25 \mathrm{mcg} /$ minute. Three hours after initiation of hemodialysis, his BP normalizes to $127 / 66 \mathrm{mmHg}$, and 2 hours later, he was weaned off norepinephrine.
Vasopressin was stopped at the completion of hemodialysis. The patient remained hemodynamically stable and medically cleared the next day.

Conclusion: Hemodialysis is a viable treatment in $\mathrm{NaN}_{3}$ ingestion with refractory vasoplegic shock.

047. Survival from Severe Methemoglobinemia After Intentional Ingestion of Sodium Nitrite. Is This an Emerging Method Recommended for Suicide?

Lien Kien Tong ${ }^{1,2}$, Hwee Min Lee ${ }^{1,2}$, Andis Graudins ${ }^{1,3}$

${ }^{1}$ Monash Toxicology Unit, Monash Health, VIC, Australia. ${ }^{2}$ Victorian Poisons Information Centre, Austin Health, VIC, Australia. ${ }^{3}$ Monash Emergency Research Collaborative, Monash University, VIC, Australia

Background: Deliberate self-poisoning with sodium nitrite is uncommon. There is limited literature reporting survival after potentially fatal concentrations of methemoglobinemia.

Methods: A 25-year-old man researched suicide methods on the internet and found a recommendation for sodium nitrite as a painless and potentially reversible form of suicide. The patient purchased $100 \mathrm{~g}$ and ingested $15 \mathrm{~g}$. He immediately had second thoughts about the attempt and called an ambulance within 10 minutes of ingestion. On paramedic arrival, he was alert, deeply cyanotic and admitted to sodium nitrite ingestion. In the emergency department, 40 minutes post-ingestion, GCS $=4$, he had ashen gray skin, pulse-oximetry saturation $60 \%$ on mask high-flow oxygen, pulse $180 \mathrm{bpm}$, and BP 91/45 $\mathrm{mmHg}$. He was emergently intubated to facilitate oxygen delivery. Peri-intubation hypotension was treated with intravenous crystalloids, metaraminol, and norepinephrine.

Results: Initial VBG revealed a methemoglobin fraction of $88 \%$, lactate $19 \mathrm{mmol} / \mathrm{L}$, and bicarbonate $14 \mathrm{mmol} / \mathrm{L}$. Three $70 \mathrm{mg}$ doses of methylene blue $(1 \mathrm{mg} / \mathrm{kg})$ were administered in the first hour post-presentation. Activated charcoal $(50 \mathrm{~g})$ was administered naso-gastrically. Methemoglobin fraction fell to $22 \%$ in the first hour and was $16 \% 2$ hours after methylene blue treatment. Hemodynamics also improved: pulse $120 \mathrm{bpm}$, BP 126/73 mmHg with successful cessation of norepinephrine infusion. Methemoglobin fraction fell to $0.9 \%$ over 30 hours. No further methylene blue was administered. He was extubated the next day without complication and discharged home after psychiatric treatment on day 4. Conclusion: Intentional ingestion of sodium nitrite is currently an uncommon method of deliberate self-poisoning. However, it is an easily accessible and unregulated salt, used in the food industry. Rapid development of methemoglobinemia can result in death without prompt medical care. In this case, early notification of the poisoning by the patient and timely methylene blue administration rapidly reversed severe methemoglobinemia and prevented irreversible end-organ dysfunction and death.

048. Severe Phenibut Withdrawal Requiring Mechanical Ventilation, High-Dose Phenobarbital, and Baclofen for Symptom Control

Rachael C Westover, Shirley Shao, Andrew F Micciche, Alexander M Sidlak, Joseph H Yanta, Joshua A Shulman

University of Pittsburgh, Pittsburgh, PA, USA

Background: Phenibut is emerging as a drug of abuse in Western countries. The withdrawal state and subsequent management is described in case reports, though treatment protocols vary widely. Two published case reports describe using phenobarbital tapers for withdrawal resistant to other treatment modalities in patients experiencing mild withdrawal. We present a case of severe phenibut withdrawal resistant to escalating doses of benzodiazepines and haloperidol requiring intubation, high-dose phenobarbital, and a baclofen taper.

Methods: This is a single patient case report. A 43-year-old male with a history of opioid use disorder presented to the emergency department for evaluation of encephalopathy after ingestion of unknown substances. The patient required a total of $14 \mathrm{mg}$ IV lorazepam, $2 \mathrm{mg}$ IM lorazepam, $50 \mathrm{mg}$ IV diazepam, $10 \mathrm{mg}$ IV haloperidol, and $10 \mathrm{mg}$ IM haloperidol 
for agitation in the first 24 hours after presentation. The patient was sedated but would have periods of psychomotor agitation, including two episodes of breaking through soft restraints. A condition was called almost 24 hours after presentation for severe agitation resistant to haloperidol and lorazepam, requiring physical and hard restraints. Gas chromatography-mass spectroscopy (GC-MS) urine drug analysis was notable for mitragynine and phenibut at that time.

Results: The patient was emergently intubated on the floor using etomidate and rocuronium. Propofol was used for post-intubation sedation. He was given a $20-\mathrm{mg} / \mathrm{kg}$ loading dose of phenobarbital and initiated on baclofen $20 \mathrm{mg}$ three times a day. Three days later, he was extubated to room air, and he did not develop subsequent episodes of agitation. The patient admitted to chronic phenibut use. He was discharged on a baclofen taper on hospital day 6 .

Conclusion: Mechanical ventilation, high-dose phenobarbital, and baclofen were required in this patient to control psychomotor agitation secondary to phenibut withdrawal.

\section{Fatal Cold Medication Poisoning in an Adolescent}

Sam T Ontiveros ${ }^{1}$, Lee Cantrell ${ }^{1,2}$

${ }^{1}$ University of California San Diego, San Diego, CA, USA. ${ }^{2}$ California

Poison Control System, San Diego Division, San Diego, CA, USA

Background: Toxicity from the intentional misuse of Coricidin products containing dextromethorphan and chlorpheniramine has been well described. Adolescents are most frequently involved and significant reported effects include CNS depression, agitation, tachycardia, and hypertension. Mortality, however, has not been well described. We report a case of an adolescent decedent with markedly elevated postmortem dextromethorphan and chlorpheniramine concentrations and no other identifiable cause of death.

Methods: A 15-year-old male with a history of "cough syrup" abuse was found deceased in bed. His social media sources included pictures of five Coricidin boxes with a stated intent to overdose. Upon autopsy, the exam showed an underweight, but well-developed teenage male. There was no evidence of trauma or other disease process. Autopsy showed mild right ventricular dilation without other cardiac abnormality. Central blood drug screening was presumptive positive for cannabinoids and phencyclidine. Peripheral blood concentrations of chlorpheniramine and dextromethorphan were 2.3 and $9.5 \mathrm{mg} / \mathrm{L}$, respectively. Gastric levels were 7 and $53 \mathrm{mg}$, respectively. No other volatiles, acidic, basic, or neutral drugs were detected.

Results: Dextromethorphan-associated mortality is rare, but reported with levels between 3.3 and $18 \mathrm{mg} / \mathrm{L}$. However, postmortem findings such as pulmonary edema are reported in these cases. Mortality from chlorpheniramine alone is poorly reported with the highest postmortem concentration at $1.1 \mathrm{mg} / \mathrm{L}$. In addition to use as an antihistamine, chlorpheniramine was studied in local and spinal anesthesia due to sodium channel blockade. QRS prolongation has not been reported following overdose, but given the markedly elevated blood concentrations found in our case, a chlorpheniramine-induced cardiac dysrhythmia cannot be ruled out.

Conclusion: Chlorpheniramine may demonstrate significant sodium channel blockade at high concentrations. It is unclear how much either dextromethorphan or chlorpheniramine contributed to the death of this adolescent, but the ingestion of large amounts may be lethal.

\section{Ingestion of a Small Quantity of "Food Grade" Hydrogen Peroxide Purchased Online}

Steven E Benatar, Mohan Punja, Richard J Kleiman WellStar Kennestone Regional Medical Center, Marietta, GA, USA

Background: Highly concentrated hydrogen peroxide $\left(\mathrm{H}_{2} \mathrm{O}_{2}\right)$ is sold in health stores and online and is advertised to have numerous health benefits. While adverse effects of ingesting $\mathrm{H}_{2} \mathrm{O}_{2}$ are highlighted in the literature, this case report demonstrates that ingesting these products in small quantities can cause concerning radiographic findings that ultimately may cause only minor adverse health effects.

Methods: This is a single patient case report. A 64-year-old male was found to have an elevated prostate-specific antigen level. He ordered "food grade $\mathrm{H}_{2} \mathrm{O}_{2}$ " online, which was advertised to help cure cancer. According to the website, this product uses "super oxygenation" to "eliminate disease and invasive organisms." The patient ingested four drops of the product. Upon drinking the solution, he had foamy emesis and presented to the emergency department (ED). His exam was remarkable for a diffusely tender abdomen. CT of the abdomen showed diffuse portal venous gas and diffuse edema of the stomach with surrounding perigastric stranding.

Results: The patient was admitted to the ICU and toxicology was consulted. Repeat CT of the chest showed improving portal venous gas. As the patient had no neurologic or cardiovascular symptoms, the decision was made to not pursue hyperbaric oxygen treatment. The patient was discharged home 2 days after admission with his symptoms resolved. Conclusion: Highly concentrated $\mathrm{H}_{2} \mathrm{O}_{2}$ is readily available for purchase, and websites claim these products treat diseases including cancer. When consumed in these high concentrations, even in very small doses, such as in this case, $\mathrm{H}_{2} \mathrm{O}_{2}$ can lead to devastating effects including gastrointestinal hemorrhage, hollow viscus rupture, and stroke. Despite dramatic radiographic findings of diffuse portal venous gas, this patient's clinical course was unremarkable. In follow-up, the patient had no lasting sequelae from the toxic ingestion.

051. Contaminated Cabin Air Events: Review of 44 Patients Presenting to a UK-Based Specialist Clinical Toxicology Outpatient Service

Carmela Maniero $^{1}$, Paul I Dargan ${ }^{1,2}$, David M Wood ${ }^{1,2}$

${ }^{1}$ Clinical Toxicology, Guy's and St Thomas' NHS Foundation Trust and King's Health Partners, London, UK. ${ }^{2}$ Faculty of Life Sciences and Medicine, King's College London, London, UK

Background: Symptoms due to contaminated cabin air events (also known as "aerotoxic syndrome") are postulated to be due to contamination of cabin air from either engine bleed air for cabin pressurization or hydraulic fluid leakage into air conditioning systems. Concern has been raised about potential exposure to a range of chemicals, including volatile hydrocarbons and organophosphates.

Research Question: To describe the clinical features of patients with concerns following a contaminated cabin air event.

Methods: This was a retrospective review of patients referred to a UK specialist clinical toxicology outpatient clinic following a contaminated cabin air event between January 2017 and October 2019. Data extracted included occupational history, aircraft type involved, symptoms, and relevant examination findings.

Results: There were 44 patients ( 26 males) with a mean $( \pm$ SD) age of 46 \pm 10 years. Eleven reported more than one contaminated cabin air event. Fourteen were flight crew, 29 were cabin crew, and 1 was a passenger. The aircraft involved was either manufactured by Airbus (total 31A319/320/321, 18; A330, 8; A380, 5) or Boeing (total 19-B737, 1; B747, 6; B757, 3; B767, 2; B777, 5; and B787, 2); 33 reported a single "contaminated air event" and 9 reported prolonged "exposure" to contaminated air (longer than an hour). Common symptoms reported by patients were headache $(n=28)$, flu-like symptoms $(n=10)$, upper respiratory tract symptoms $(n=25)$, fatigue/lethargy $(n=23)$, gastrointestinal symptoms $(n=19)$, paraesthesia $(n=18)$, breathlessness $(n=16)$, and memory problems $(n=7)$. Five patients had abnormal neurological examination (four cerebellar signs), and one patient had abnormal cardiovascular examination.

Conclusion: Patients with concerns following a potential contaminated cabin air event present with a range of symptoms, which may be prolonged following an event. Although the concern is around engine bleed air as the 
cause, two patients reported concerns/symptoms related to Boeing 787 aircraft which does not use engine bleed air for cabin pressurization.

\section{Severe Occupational Lead Exposure in an Adult}

Elizabeth Black ${ }^{1}$, Linda Forst ${ }^{2}$, Jerrold B Leikin ${ }^{3}$

${ }^{1}$ Toxikon Consortium-John Stroger Jr Hospital, Chicago, IL, USA.

${ }^{2}$ University of Illinois School of Public Health, Chicago, IL, USA.

${ }^{3}$ NorthShore University Health System-OMEGA, Glenview, IL, USA

Background: Adult lead toxicity in the USA is relatively rare (2.8 cases per 100,000 in 2016). We report on an adult with significant occupational lead toxicity, with significant consequences.

Methods: This is a single case report. Patient presented to the emergency department twice for constant, nonexertional left-sided chest pressure, limb numbness, ataxia, joint pain, headache, and a mild right hand tremor. $\mathrm{He}$ underwent a negative cardiac work-up, received a diagnosis of noncardiac, "atypical" chest pain, and was referred to an outpatient clinic. Prior to followup, the patient performed an internet search; due to his occupational history of removing paint via abrasive blasting for 8 months, with limited respiratory protection, in a building built in 1921, he requested lead testing. Blood lead level (BLL) returned at $135 \mathrm{mcg} / \mathrm{dL}$.

Results: Patient was admitted, started on dimercaprol and calcium disodium EDTA for 4 days, then transitioned to oral succimer for a total of four 19-day courses over 7 months. BLL decreased to $7.4 \mathrm{mcg} / \mathrm{L}$ after 26 months, and his symptoms improved, other than a persistent headache, which was diagnosed as an ocular migraine (ophthalmologic evaluation was unremarkable).

Conclusion: This patient was not provided appropriate personal protective equipment and did not undergo appropriate lead surveillance, as defined by OSHA (Title 29, section 1926.62); OSHA ultimately fined three responsible companies for 72 violations. The patient has had extensive financial and family difficulties due to the exposure and has not been able to secure work from the same or any other construction contractor. Adult occupational lead exposure may be an impostor for cardiovascular disease. Engaging OSHA early is crucial to protecting the patient's and co-workers' health; construction workers are particularly vulnerable to retaliatory firing since they need to be rehired for each job. Prolonged unemployment in this scenario adds financial, psychological, social, and other collateral adverse effects.

053. Descriptive Epidemiology of Clinically Significant Occupational Poisonings in the United States, 2008-2018

John W Downs, Brandon K Wills, Kirk L Cumpston, S. Rutherfoord Rose

Virginia Commonwealth University Health System, Richmond, VA, USA

Background: Lists of common occupational toxins include many historical toxins previously responsible for poisoning in the decades prior to worker protective laws and regulations. Little recent information has been published evaluating the current causes of work-related acute exposures reported to poison centers.

Research Question: What are the most common toxins, routes of exposure, and risk factors for clinically significant occupational poisonings? Methods: This was a retrospective cohort study using electronic data from the AAPCC National Poison Data System (NPDS) and open source data from the United States Bureau of Labor Statistics (BLS). The NPDS was queried for all cases with exposure reason coded as "UnintentionalOccupational" for the period 1 January 2008 to 1 January 2018. A case of clinically significant occupational poisoning (CSOP) was defined as a case with moderate or severe clinical effects reported, to include fatal cases. A descriptive analysis was conducted using unadjusted odds ratios to assess the strength of association between main variables of interest and CSOP.

Results: There were 329,437 exposure calls available for analysis. Of these, 54,254 were considered CSOP and included 196 deaths.
The top 5 fatality-associated toxins were hydrogen sulfide, ammonia, carbon monoxide, simple asphyxiants, and chlorines. Fatalities were 3.7 times (OR 3.7; 95\% CI 2.2-6.4) more likely to be men and 5.7 times (OR 5.7; 95\% CI 4.0-8.1) more likely to have had an inhalational exposure, compared to those workers with CSOP who were not fatalities. The period crude fatality rate was 7.2 deaths per $100,000,000$ worker-years. The crude rate of CSOP was 2 per 100,000 worker-years.

Conclusion: Simple and chemical asphyxiants continue to be responsible for occupational-related significant poisonings and fatalities. Workplace education and proper preventive measures devoted to inhalational toxins and respiratory protection are opportunities for improvement.

\section{Methylene Chloride or Messed Up Machine? A Case Series}

Stephen L Thornton ${ }^{1,2}$, William Barkman ${ }^{3}$, Tony Rianprakaisang ${ }^{2}$ ${ }^{1}$ University of Kansas Health System Poison Control Center, Kansas City, KS, USA. ${ }^{2}$ University of Kansas Health System Department of Emergency Medicine, Division of Medical Toxicology, Kansas City, KS, USA.

${ }^{3}$ University of Kansa Health System Pulmonary and Critical Care Medicine, Kansas City, KS, USA

Background: Methylene chloride (MC) exposures can result in elevated carboxyhemoglobin $(\mathrm{COHgb})$ levels. We report on three patients misdiagnosed as $\mathrm{MC}$ exposures due to elevated $\mathrm{COHgb}$ levels from a faulty co-oximeter.

Hypothesis: Co-oximeter malfunction may lead to misdiagnosis of a $\mathrm{MC}$ exposure.

Methods: At work, three women (ages 27, 37, and 50) were exposed to unknown fumes for 3 hours. They developed dizziness, headache, nausea, nearsyncope, and trouble ambulating. In the $\mathrm{ED}$, initial $\mathrm{COHgb}$ levels were as follows: 27 -year old $-7.5 \%, 37$-year old $-8.7 \%$, and 50 -year old $-6.1 \%$. The 27 - and the 50-year-old patients were nonsmokers. The 37-year old smoked 3 days prior. They were placed on $100 \% \mathrm{O}_{2}$ and admitted; $100 \% \mathrm{O}_{2}$ was continued for the first 2 days of admission. The 27- and the 50 -year old's $\mathrm{COHgb}$ peaked at $8.2 \%$ ( 84 hours post-exposure) and $10.5 \%$ (110 hours post-exposure), respectively. The 37-year old's initial COHgb was her highest, but she had a level of $8.5 \%$ at 51 hours post-exposure. Based on these labs, a methylene chloride exposure was diagnosed. They were discharged with improved symptoms but continued elevated $\mathrm{COHgb}$ levels after a 5-day hospital stay. An OSHA investigation could not identify any source of carbon monoxide (CO) or MC but did find the hospital's co-oximeter to be faulty. When rerun on a functioning machine, initially abnormal COHgb samples were found to be normal.

Discussion: $\mathrm{MC}$ is a common solvent that is metabolized to $\mathrm{CO}$ with resultant elevations in $\mathrm{COHgb}$. The kinetics of MC-associated $\mathrm{COHgb}$ differs from environmental $\mathrm{CO}$ exposures and can rise in the face of oxygen therapy, as occurred in these cases. However, the pattern and duration of abnormal $\mathrm{COHgb}$ seen in these cases was atypical, even for large methylene chloride exposures. Conclusion: Co-oximeter malfunction should be in the differential diagnosis for possible $\mathrm{MC}$ exposures.

055. Steroid Statistics: a Regional Review of the Use of Systemic Steroids in the Treatment of Electronic Vaping-Associated Lung Injuries (EVALI)

Mary Ellen R Billington, Kim Aldy, Paul Wax, Dazhe J Cao, On Behalf of the ToxIC Investigators Consortium (ToxIC)

UT Southwestern, Dallas, TX, USA

Background: Nicotine vaping was introduced as a theoretically "cleaner" alternative to traditional cigarette smoking. In recent years, novel delta-9-tetrahydrocannabinol (THC) oil cartridges have enjoyed significant popularity among young adults. This year, a striking number of electronic vaping-associated lung injury (EVALI) cases have been reported nationally. Although health authorities are working diligently to define 
the disease, definite information regarding etiology and treatment remains a subject of clinical intrigue. Currently, the CDC makes the nonspecific recommendation that steroids may represent a treatment consideration; however, specific evidence and dosing regimens are not reported.

Hypothesis: We aim to characterize the patterns of systemic steroid use in the treatment of EVALI patients at our institutions.

Methods: Institution-specific data were obtained as a subset from the national ToxIC Registry. A total of 27 EVALI cases were identified and individually analyzed. All cases had been seen at bedside by medical toxicologists at our associated academic facilities in a large metropolitan city, from August through October of 2019. One case was excluded due to incomplete data.

Results: A significant majority of patients (88\%) were treated with systemic steroids. Of the patients administered steroids, $78 \%$ received treatment via intravenous administration. The majority of treated patients received a steroid taper. Remarkable heterogeneity exists among steroid-dosing regimens. Treatment duration ranged from 2 days to several weeks; $50 \%$ of cases involved pediatric patients (age $<19$ ), with overall age ranges $13-55$. All pediatric patients and patients age $>30$ years were treated with systemic steroids. Of treated patients, only $3(11 \%)$ received no more than $60 \mathrm{mg}$ prednisone per day.

Conclusion: This report characterizes the use of systemic steroids in the clinical treatment of EVALI. Steroid use was highly prevalent, predominantly via the intravenous route of administration. Dosing was characteristically heterogeneous, though tapering was common among those treated.

\section{Toxicology Investigators}

\section{Ozone Gas Embolism}

Lauren T Murphy ${ }^{1,2}$, Jennifer S Love ${ }^{1,2}$, John A Thompson ${ }^{1,2}$, Nate J McKeown ${ }^{1,2}$

${ }^{1}$ Oregon Health \& Science University, Portland, OR, USA. ${ }^{2}$ Oregon Poison Center, Portland, OR, USA

Background: Ozone $\left(\mathrm{O}_{3}\right)$ is an alternative medicine used for its purported antimicrobial, antifungal, and anti-inflammatory properties. We report a gas embolism that occurred during $\mathrm{O}_{3}$ autohemotransfusion resulting in acute lung injury (ALI).

Methods: This is a single patient case review. A 52-year-old female received $\mathrm{O}_{3}$ autohemotherapy in a clinic for treatment of a chronic, poorly characterized neurologic disorder. During transfusion of ozonated blood back to the patient, she suddenly lost consciousness. The provider estimated $250-500 \mathrm{~mL}$ of $\mathrm{O}_{3}$ gas had been injected from the generator intravenously. The patient was rolled to her side, given $1 \mathrm{mg}$ intravenous epinephrine, and EMS transported the patient in Trendelenburg to the nearest emergency department. Upon arrival, the patient was awake but confused with normal vital signs and a nonfocal neurologic exam. A head CT showed no air embolus. As the patient's mental status improved, she developed increasing oxygen requirements. A chest X-ray displayed bilateral reticulonodular infiltrates with small pleural effusions with concern for pulmonary edema, and the patient required noninvasive positive pressure ventilation for ALI for 4 hours. A noncontrast CT of the chest, abdomen, and pelvis revealed no air emboli. Her lung injury resolved, and no further focal neurologic deficits were noted.

Discussion: This is the second case describing an $\mathrm{O}_{3}$ embolism. A previous fatal case described only autopsy findings including a patent foramen ovale and multiple diffuse air emboli. $\mathrm{O}_{3}$ is an oxidant gas that is known to create reactive oxygen species and cause lipid peroxidation in the body. The hypothesized mechanism behind $\mathrm{O}_{3}$-mediated lung injury involves sensitization of opioid-mediated C-fibers and production of neutrophil and monocyte chemoattractants and subsequent inflammatory cascade. Documented results include dyspnea and reversible reduction in vital capacity.

Conclusion: Ozone air embolism may be associated with ALI and is a potentially life-threatening complication of ozone autohemotherapy.

057. Retrospective Poison Center Validation of the Negative Predictive Value of Acetaminophen Transaminase Multiplication Product of $1500 \mathrm{mg} / \mathrm{L} \mathrm{IU/L}$

Kartik R Shah ${ }^{1}$, Michael C Beuhler ${ }^{2}$

${ }^{I}$ Atrium Health's Carolinas Medical Center, Charlotte, NC, USA. ${ }^{2}$ North Carolina Poison Control, Charlotte, NC, USA

Background: Retrospective studies have suggested that the multiplication product of the acetaminophen (APAP) level and the higher transaminase (AST or ALT) has a strong correlation with the probability a patient will develop hepatotoxicity. A multiplication product under $1500 \mathrm{mg} / \mathrm{L} \mathrm{IU} / \mathrm{L}$ has a negative correlation to hepatotoxicity. However, this threshold has never been evaluated in a poison center cohort.

Hypothesis: The multiplication product threshold of $1500 \mathrm{mg} / \mathrm{L}$ IU/L has a high negative predictive value for hepatotoxicity.

Methods: This is a retrospective study of consecutive patients reported to a large regional poison center. A search of the poison center's electronic medical record system (January 1,2017-December 31,2017) was used to identify all patients referred to a healthcare facility with an acetaminophen generic product code. Each case's text notes was read, and the initial APAP level, transaminase level drawn closest to the APAP level, and peak transaminase level were collected. The multiplication product was calculated if an APAP level and transaminases were numerically recorded. Patients with undetectable APAP levels were assumed to have a level of $9.9 \mathrm{mg} / \mathrm{L}$ for the multiplication product. Cases with a multiplication product $<1500 \mathrm{mg} / \mathrm{L} \mathrm{IU} / \mathrm{L}$ were evaluated for the development of hepatotoxicity, defined as peak transaminase $\geq 1000 \mathrm{IU} / \mathrm{L}$. The negative predictive value for hepatotoxicity was calculated.

Results: There were 2231 patients identified. Of these, 537 patients had a multiplication product $<1500 \mathrm{mg} / \mathrm{L} \mathrm{IU} / \mathrm{L}$, with 279 patients having a detectable APAP level and 258 patients having an undetectable APAP level. One patient became hepatotoxic, leading to a negative predictive value of $99.8 \%$.

Conclusion: In a poison center retrospective evaluation, a multiplication product threshold of $1500 \mathrm{mg} / \mathrm{L} \mathrm{IU} / \mathrm{L}$ had a high negative predictive value for hepatotoxicity, but it was not $100 \%$.

058. Inhalational Abuse of Keyboard Cleaner Presenting as Anteroseptal ST-Elevation Myocardial Infarction Mimic and Leading to Fatal Dysrhythmias

Nixon Nguyen, Richard J Kleiman, Mohan Punja, Mene Demestihas WellStar Kennestone Regional Medical Center, Marietta, GA, USA

Background: Fluorinated hydrocarbon inhalation can cause dysrhythmias and possibly cardiomyopathy, hepatic injury, and renal injury.

Methods: This is a single patient chart review.

Results: A 28-year-old male with a polysubstance abuse history presented to the ED after being found unresponsive. He was alert when EMS arrived and complained of chest pain. He admitted using approximately 20 cans/day of Dust-Off®, a difluoroethane hydrocarbon keyboard cleaner, for the prior 11 days since discharge from drug rehabilitation. $\mathrm{He}$ denied any other substances during this period. Labs were significant for a Troponin T of $5.24 \mathrm{ng} / \mathrm{mL}$, potassium of $8.4 \mathrm{mmol} / \mathrm{L}$, creatinine of $4.5 \mathrm{mg} / \mathrm{dL}$, and CK of $6440 \mathrm{IU} / \mathrm{L}$. The ECG showed $3+\mathrm{mm}$ ST elevation in leads V1-V3 with reciprocal changes in V4-V6 and he was taken for PCI. Catheterization showed $0 \%$ stenosis of all coronary arteries and an echocardiogram showed a $10 \%$ ejection fraction. During hospitalization, he developed episodes of ventricular tachycardia. Metoprolol, 
amiodarone, and lidocaine were tried without success, and 5 days later, he developed ventricular fibrillation and died. The diagnosis was myocardial injury and cardiomyopathy secondary to inhalational abuse of fluorinated hydrocarbons.

Discussion: Fluorinated hydrocarbon inhalation typically presents with adverse effects on the central nervous system, in addition to dysrhythmias and renal injury. There are case reports describing cardiomyopathy after similar exposures; however, those patients have recovered cardiac function and done well. Given the acute, intense exposure after a period of inpatient sobriety, and lack of prior cardiac disease in this young patient, we surmise a causal relationship between the hydrocarbon abuse and the outcome. The unique ECG findings and outcome have not been described in prior case reports.

Conclusion: In this case report, fluorinated hydrocarbon inhalation led to a severe, likely acute cardiomyopathy manifested by syncope, an ECG pattern mimicking acute myocardial infarction, and ultimately fatal, refractory ventricular dysrhythmias.

\section{Day 2: Platforms, Abstracts 059-062}

059. What's in an E-cigarette? Preparation and Identification of Adducts Formed Between Flavoring Molecules and Carrier Fluids

Jody Morgan, Celine Kelso, Tristan Agostini, Alison L Jones

University of Wollongong, Wollongong, NSW, Australia

Background: Electronic cigarette (e-cig) sales have been on an upward trajectory for the past 10 years with an increase of $47 \%$ between 2016 and 2017 (from U.S. \$896 million to U.S. \$1318 million annual U.S. sales). The recent identification of e-cigarette or vaping product use-associated lung injury (EVALI) which has affected $>1300$ people in the USA has increased the urgency to examine e-cig fluids. A study examining the content of 49 e-cig fluids available to Australian consumers in 2019 led to the identification of possible adducts being formed in situ.

Methods: This is a research study examining the formation of new chemical components produced in situ when commonly used flavoring molecules are added to e-cig carrier fluids propylene glycol (PG) and vegetable glycerin (VG). Solutions of three common carbonyl-containing flavoring molecules, vanillin, ethyl vanillin, and ethyl maltol, were prepared in PG, VG, and 50:50 PG:VG. These nine solutions were synthesized in triplicate and sampled daily for 1 week, then at regular intervals for a further 2 weeks, and the samples were diluted 200-fold in LCMS grade methanol and examined by GC-MS.

Results: Vanillin, ethyl vanillin, and ethyl maltol were shown to react with both PG and VG to produce adducts in solution. All adducts were shown to appear within 1 week of sample preparation. Vanillin adduct formation with PG produces two peaks on GC-MS which are diastereomers of vanillin propylene glycol acetal. Vanillin adduct formation with VG produces four peaks on GC-MS two of which are the diastereomers resulting from a 1,2-addition and two peaks which are from the corresponding 1,3-addition.

Conclusion: E-cig fluids are not chemically stable with in situ adducts forming between carbonyl-containing flavoring molecules and carrier fluid components. Additional work needs to be performed in this area to identify the potential toxicity of these molecules.

060. Utilization of Bedside Medical Toxicology Consultation Service May Lead to Hospital Cost Savings

Todd M Phillips ${ }^{1,2}$, Chet D Schrader ${ }^{1,3}$, James P D’Etienne ${ }^{1,2}$

${ }^{1}$ JPS Health Network, Fort Worth, TX, USA. ${ }^{2}$ Integrative Emergency Services, Dallas, TX, USA. ${ }^{3}$ Integrative Emergency Services, Fort Worth, TX, USA
Background: Most intoxicated patients in the USA do not have access to bedside medical toxicologist (MT) consultation. While some data exists, there is an overall paucity of research comparing the cost to the hospital by utilizing a bedside MT compared to without.

Hypothesis: Bedside MT consultation will have an overall cost savings to the hospital.

Methods: This is a case-control series conducted at a large, county, academic, level 1 trauma center single site during February to December of 2018. A single MT provided bedside consultation at the discretion of the Emergency Medicine (EM) team. The EM team would formulate their own normal care path for the patient without consultation. Independently, the MT would formulate their own care plan which was then implemented on all patients. Cost savings were analyzed comparing the EM teams anticipated course versus the MT-implemented plan. Cost savings were objective measures including hospital length of stay days, ICU length of stay days, and medication administration costs. Costs of MT consultations would be \$275/patient and were based on a fair market analysis for a flat fee regardless of acuity.

Results: Seventy-one consultations were obtained. The EM team and MT had the same plan for 45/71 (63\%) of the patients. However, 26/71 (37\%) patients had care plans that differed. Comparing the care plan of the EM team versus the MT-implemented plan, there was a cost savings of $\$ 90,742$, with consultation fees being $\$ 19,525$. After this adjustment for all 71 consultations, the total cost savings would be $\$ 71,217$, or $\$ 1003$ per patient. All patients were followed via chart review until discharge and there were no adverse outcomes.

Conclusion: Bedside MT consultation for the acutely intoxicated patient may lead to cost savings to the hospital by reducing length of stay, ICU admission days, and medication administration.

061. Blood Cannabinoid Molar Metabolite Ratio Is Superior to Blood THC as an Indicator of Recent Cannabis Smoking

Michael J Kosnett ${ }^{1,2}$, Ashley Brooks-Russell ${ }^{1}$, George S Wang ${ }^{2,3}$, Greg Dooley $^{4}$, Gary Milavetz ${ }^{5}$, Kyle Friedman ${ }^{2}$, Susan Heard ${ }^{2}$, Chelsey Thibodeaux ${ }^{2}$, John Schwarz ${ }^{2}$

${ }^{1}$ Colorado School of Public Health, Aurora, CO, USA. ${ }^{2}$ Rocky Mountain Poison \& Drug Safety, Denver, CO, USA. ${ }^{3}$ University of Colorado School of Medicine, Denver, CO, USA. ${ }^{4}$ Colorado State University, Fort Collins, CO, USA. ${ }^{5}$ University of Iowa College of Pharmacy, Iowa City, IA, USA

Background: More than a dozen U.S. states have a legal threshold for whole blood THC blood concentration that establishes a presumption of driving under the influence of cannabis. However, the temporal relationship between last use of cannabis, psychoactive effects, and blood THC concentration may vary with the cannabis use history of individuals. Particularly for daily users, THC may be detectable in blood after acute psychoactive effects have resolved.

Research Question: How will the blood cannabinoid molar metabolite ratio (MMR) of (THC + THC-OH)/THC-COOH compare to blood THC alone as an indicator of recent cannabis smoking?

Methods: As part of an observational study of driving and psychomotor performance, whole blood cannabinoids, including THC, THC-OH, and THC-COOH, were measured by LC/MS/MS at baseline and 30 minutes after starting a 15-minute interval of ad libitum smoking cannabis flower (mean total THC 21.7\%) in occasional (1-2 days/week over the past 30 days) $(n=27)$ and daily cannabis smokers $(n=32)$.

Results: Median and IQR for THC: occasional user baseline $(0.0 \mathrm{ng} / \mathrm{ml} ; 0.0-0.0 \mathrm{ng} / \mathrm{ml})$, post-smoking (4.1 ng/ml; 2.4 $7.1 \mathrm{ng} / \mathrm{ml})$; daily user baseline $(2.7 \mathrm{ng} / \mathrm{ml}, 1.2-5.1 \mathrm{ng} / \mathrm{ml})$; postsmoking (21.3 ng/ml; 8.7-42.5 ng/ml). MMR: occasional user baseline $(0.0,0.0-0.0)$, post-smoking $(0.71,0.58-1.02)$; daily user baseline $(0.11,0.07-0.17)$, post-smoking $(0.53,0.36-0.61)$. In ROC curve analysis, blood THC of 6.4 yielded optimal $64 \%$ sensitivity, $91 \%$ specificity, and $78 \%$ accuracy for identification of recent cannabis smoking; MMR of 0.25 yielded optimal 
sensitivity of $80 \%$, specificity of $99 \%$, and $89 \%$ accuracy for recent cannabis smoking (Accuracy $=$ correct assessments $/$ total assessments).

Conclusion: In a population that included occasional and daily users, the blood cannabinoid molar metabolite ratio $[\mathrm{MMR}=(\mathrm{THC}+\mathrm{THC}-\mathrm{OH}) /$ THC-COOH] threshold of 0.25 had superior accuracy than whole blood $\mathrm{THC}$ as an indicator of recent cannabis use.

\section{Testing the Accuracy of a Commercially Available Breathalyzer} Used by Law Enforcement

Ryan Marino ${ }^{1,2}$, Alexander Sidlak ${ }^{3}$, Anthony Pizon $^{3}$

${ }^{1}$ University Hospitals, Cleveland, OH, USA. ${ }^{2}$ Case Western Reserve University School of Medicine, Cleveland, OH, USA. ${ }^{3}$ University of Pittsburgh School of Medicine, Pittsburgh, PA, USA

Background: Breathalyzer testing for alcohol intoxication is a mainstay of law enforcement. Advances in electrochemical testing techniques have reportedly made breathalyzer results more accurate, and some models, like the BACtrack ${ }^{\circledR}$ S80 Professional Breathalyzer, are approved for this testing by the US Department of Transportation, the National Highway Traffic Safety Administration, and the US Food \& Drug Administration. Hypothesis: Alcohol testing by breathalyzer analysis does not accurately reflect blood ethanol levels.

Methods: We conducted a prospective cohort study of 22 healthy volunteers given weight-based doses of ethanol over 2 hours to achieve an ethanol level greater than $200 \mathrm{mg} / \mathrm{dL}$. Breath alcohol analysis using the BACtrack ${ }^{\circledR}$ S 80 was performed with concurrent serum ethanol testing at $0,2,4$, and 6 hours. The breathalyzer device used fuel cell technology, where alcohol in a breath sample is oxidized to generate electrical current, which is measured and converted to a reported value. Serum ethanol was measured using quantitative gas chromatography.

Results: All 22 subjects were able to successfully use the breathalyzer and calibrated the device. There were no false-positive results. Sixty positive breathalyzer results were obtained concurrently with serum levels. The average difference between concurrent measurements was $39 \mathrm{mg} / \mathrm{dL}$ (standard deviation $32 \mathrm{mg} / \mathrm{dL}$ ). Using paired $t$ test to compare the two measurements demonstrated that breathalyzer analysis gives significantly lower results than serum ethanol levels $(p<0.01)$. Applying multiple legally accepted conversion factors $(1.12,1.18)$ for converting serum ethanol concentration to whole blood ethanol concentration still resulted in breathalyzer values that were significantly lower (mean difference $17.1 \mathrm{mg} / \mathrm{dL}, 8 \mathrm{mg} / \mathrm{dL})$ from blood levels $(p<0.01, p=0.01)$. Conclusion: Breath alcohol measurement is used frequently with legal implications. While fuel cell electrochemical testing is considered an accurate form of measurement and endorsed by multiple official agencies for legal testing, it does not accurately correlate to serum ethanol levels or serum ethanol levels using a correction factor.

Day 2: Moderated Posters, Abstracts 063-068

\section{In-Hospital Use of Medication for Addiction Treatment for Opioid Use Disorder}

Skyler H Kessler, David B Liss, Evan S Schwarz

Washington University in St. Louis School of Medicine, St. Louis, MO, USA

Background: Over 47,000 Americans died from an opioid related overdose in 2017, and approximately 2.1 million suffer from opioid use disorder (OUD). Currently, few hospitals provide medication for addiction treatment (MAT) to patients with OUD. More data are needed to inform proper in-hospital initiation of MAT.
Research Question: Does choice of in-hospital MAT (buprenorphine vs. methadone) for OUD influence length of retention in outpatient OUD treatment?

Methods: This was a retrospective cohort analysis of patients who received a consult for OUD from the Division of Toxicology at Barnes Jewish Hospital. Each patient's medical record was reviewed to determine whether they received MAT and were referred to the program Engaging Patients in Care Coordination (EPICC), an initiative that connects hospitalized patients with OUD to outpatient care. We utilized data from December 2016 to May 2019, supplied by EPICC, to determine rates of retention in outpatient treatment after hospital discharge. Patients were stratified by the last form of MAT they received in the hospital (methadone vs. buprenorphine); a chi-squared test was performed to quantify differences in outcome.

Results: Of 196 total patients, 113 received MAT and referral to EPICC, with $68 \%$ receiving buprenorphine and $32 \%$ receiving methadone as their last form of MAT prior to discharge. The rate of retention in outpatient treatment for patients who received buprenorphine was 36,26 , and $14 \% \mathrm{~m}$ and for patients who received methadone, it was 47,44 , and $39 \%$ at 2 weeks, 30 days, and 12 weeks, respectively. There was no statistically significant difference in retention rate at 2 weeks or 30 days. The difference in retention at 12 weeks was statistically significant $(p<0.01)$.

Conclusion: Patients who received methadone in-hospital were more likely to remain in outpatient treatment than those who received buprenorphine.

064. Acute Opioid OD in ED Patients Reporting Prior MOUD Use: Prevalence, Misuse, and Its Effect on Overdose Severity

Ishak Nobel ${ }^{1}$, Lisa R Allen ${ }^{2}$, Lynne D Richardson ${ }^{2}$, Kavey Vidal ${ }^{2}$, Alex F Manini $^{2,3}$

${ }^{1}$ College of Medicine, SUNY Upstate Medical University, Syracuse, NY, USA. ${ }^{2}$ Department of Emergency Medicine, Icahn School of Medicine at Mount Sinai, New York, NY, USA. ${ }^{3}$ Division of Medical Toxicology, Icahn School of Medicine at Mount Sinai, Elmhurst Hospital Center, New York, $N Y, U S A$

Background: Medications for opioid use disorder (MOUD) reduce opioid overdose deaths; however, in ED patients, its prevalence, misuse, and effects on overdose severity are unclear. Therefore, we examined trends in baseline MOUD use in this population, and hypothesized that MOUD use should increase over time with corresponding decreases in overdose severity.

Methods: This was a secondary data analysis from a prospective observational cohort of ED patients with drug overdoses at two tertiary-care hospitals from 2015 to 2019. Patients with confirmed opioid overdose were included. Clinical data and prior MOUD use were collected from medical records using trained abstractors blinded to study hypotheses. Overdose severity was defined as hospital length of stay (in days) and in-hospital mortality. Prevalence, standard error (SE), and odds ratios (OR) with 95\% confidence intervals (CI) were calculated using SPSSv24. Results: Of the $2829 \mathrm{ED}$ patients with acute drug overdose enrolled in the parent cohort, 696 patients were included (mean age $45,70 \%$ male), and $118 / 696(16.95 \%)$ patients were prescribed any MOUD (methadone $=$ 103 , buprenorphine $=14$, naltrexone $=3$ ). MOUD prevalence was significantly higher in 2018 and 2019 compared to 2016 (20.1 and 27.8 vs. $8.8 \%, p<0.05)$. Odds of drug misuse were significantly higher for methadone (OR 4.6, CI 2.9-7.2) and lowest for buprenorphine (OR 1.35, $p=$ NS). Mean LOS was higher for methadone (3.08 \pm 0.55$)$ compared to buprenorphine $(2.00 \pm 0.59)$ and naltrexone $(2.00 \pm 0, p=\mathrm{NS})$. Odds of death were lower for patients on any MOUD (OR 0.70, CI 0.09-5.7), but highest in the methadone group (OR 0.82, CI 0.1-6.7).

Conclusion: We found that MOUD prevalence significantly increased over the study period. Odds of prescription drug misuse were significantly higher for patients taking methadone compared to other MOUD. Overdose severity was lower in patients with any prior MOUD use; however, overdose severity was higher in methadone patients compared 
to buprenorphine patients.

\section{Impact of Prescription Drug Monitoring Programs on} Adolescent and Young Adult Opioid Prescribing

Michael S Toce, Karen Olson, Michael C Monuteaux, Florence T Bourgeois

Boston Children's Hospital, Boston, MA, USA

Background: Prescription Drug Monitoring Programs (PDMPs) are staterun databases that aim to promote judicious use of controlled substances and reduce misuse of these drugs. The effects of PDMPs on opioid prescribing to adolescent and young adult patients have not been described.

Methods: We conducted state-level interrupted time series analyses with monthly repeated measures to examine the impact of PDMPs on the rate of opioid prescribing among adolescents (13-18 years) and young adults (19-25 years) from January 2008 to June 2017. Prescription data were obtained from a commercial health insurance dataset covering 68 million patients nationally. We focused our analysis on 29 states that implemented a PDMP during our study period and provided pre- and postimplementation data.

Results: There were $1,683,057$ opioid prescriptions filled by 485,291 adolescents and 1,197,766 young adults during the study period. The mean prescribing rate per month was 9212 prescriptions per 1,000,000 adolescents and 18,492 per 1,000,000 young adults. Among adolescents, prior to PDMP implementation, prescribing rates were increasing by 14.5 (95\% CI, 1.8, 27.2) prescriptions per 1,000,000 adolescents per month. After implementation, rates were decreasing by 22.8 (95\% CI, 15.1, 30.6) prescriptions per $1,000,000$ per month, representing an absolute change of $-37.3(95 \% \mathrm{CI},-51.5,-23.1)$ prescriptions per $1,000,000$ adolescents per month. Prescribing rates were also increasing for young adults in the pre-implementation period, at 74.1 (95\% CI, 46.2, 102.0) prescriptions per $1,000,000$ young adults per month. After implementation, rates were decreasing by 63.8 (95\% CI, 47.9, 79.7) prescriptions per $1,000,000$ per month, representing an absolute change of -137.9 (95\% CI, -170.2 , - 105.6) prescriptions per 1,000,000 young adults per month.

Conclusion: Implementation of state-level PDMPs was associated with a significant reduction in opioid prescriptions filled by adolescent and young adult patients. Further work should explore whether this is associated with a decrease in opioid misuse and overdoses.

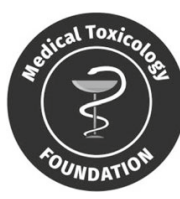

This research was supported by the 2019 Medical Toxicology Foundation Innovative Research and Teaching Award.

066. Access to Medication-Assisted Treatment Among Residential Treatment Programs in the United States

Robert S Iannaccone ${ }^{1}$, Christine U Ramdin ${ }^{1}$, Jeanmarie Perrone ${ }^{2}$, Lewis S Nelson ${ }^{1}$

${ }^{1}$ Rutgers New Jersey Medical School, Newark, NJ, USA. ${ }^{2}$ University of Pennsylvania Perelman School of Medicine, Philadelphia, PA, USA

Background: Addressing the opioid epidemic requires an expansion of accessible, evidence-based treatment, particularly medication-assisted treatment (MAT). The common practice of residential "detoxification" using methadone or buprenorphine to manage opioid use disorder (OUD) without offering sustained treatment is dangerous and counters the evidence basis for MAT. We evaluated the accessibility and prevalence of MAT utilization among residential treatment facilities in the USA.
Methods: This is a "secret shopper" study in which treatment facilities were contacted by telephone during business hours. We directly called 99 residential treatment facilities randomly selected from the SAMHSA Facility Navigator website with a scripted case-based questionnaire about accessing treatment and the types of treatment offered at the facility. The survey was designed to mimic a conversation between intake personnel and a family member of a patient with OUD.

Results: Of 99 treatment facilities contacted across the USA, study investigators were only able to obtain a direct response from $66(66.7 \%$, $95 \%$ CI $[0.56,0.76])$. Of these, MAT was offered at $42(63.6 \%, 95 \% \mathrm{CI}$ $[0.52,0.74])$ facilities for detoxification and only $5(11.9 \%, 95 \%$ CI $[0.05$, $0.25])$ for ambulatory maintenance therapy. Buprenorphine was offered in $35(53.0 \%, 95 \%$ CI $[0.41,0.65])$. Among the systematic hurdles noted were the use of nonhuman automated menu by 20 facilities $(30.3 \%, 95 \%$ CI $[0.20,0.43])$, inability to address the investigator's questions in 11 $(16.7 \%, 95 \%$ CI $[0.09,0.28])$, and the ability to obtain outcomes data from only $13(19.7 \%, 95 \%$ CI [0.11, 0.32]).

Conclusion: Many treatment facilities have not adopted evidence-based treatment strategies for OUD. It is difficult to contact treatment facilities and obtain accurate information regarding OUD treatment. SAMHSA should provide guidance about offering evidence-based treatment including a preference for centers that provide sustained MAT and to recommend against abstinence-based or detoxification therapy.

067. Opioid Overdose Deaths with Buprenorphine Detected in Postmortem Toxicology: a Retrospective Analysis

Rachel S Wightman ${ }^{1,2}$, Jeanmarie Perrone ${ }^{3}$, Rachel Scagos ${ }^{4}$, Maxwell Krieger $^{5}$, Lewis S Nelson ${ }^{6}$, Brandon DL Marshall ${ }^{5}$

${ }^{I}$ Alpert Medical School of Brown University, Providence, RI, USA. ${ }^{2}$ Brown Emergency Medicine, Providence, RI, USA. ${ }^{3}$ Perelman School of Medicine, University of Pennsylvania, Philadelphia, PA, USA. ${ }^{4}$ Rhode Island Department of Health, Providence, RI, USA. ${ }^{5}$ Brown University School of Public Health, Providence, RI, USA. ${ }^{6}$ Rutgers New Jersey Medical School, Newark, NJ, USA

Background: Buprenorphine may be superior to methadone as it is a unique $\mu$-opioid receptor partial agonist with avid receptor binding, nominal euphoric reward, and a ceiling effect on sedation and respiratory depression. Despite a pharmacologic profile that enhances safety, cases of fatal opioid overdose with buprenorphine detected on postmortem toxicology are reported, but details of these cases in the literature are limited.

Research Question: What is the profile of overdose fatalities when buprenorphine is detected on postmortem toxicology and how does this compare to opioid overdose deaths without buprenorphine?

Methods: This is a retrospective review of opioid-involved drug overdose fatalities in Rhode Island (RI) from 2016 to 2018 using the RI Department of Health State Unintentional Drug Overdose Reporting Surveillance (SUDORS) database. Deaths with buprenorphine on descriptive toxicology versus opioid-involved overdose deaths without buprenorphine were compared to assess the type and number of coingestants contributing to death.

Results: Of 534 opioid-involved deaths, 29 (5.4\%) included buprenorphine and/or norbuprenorphine on toxicology. The most frequent coingestants were as follows: fentanyl $(75.9 \%)$, norfentanyl (72.4\%), cocaine $(41.4 \%)$, benzoylecgnonine $(41.4 \%)$, cannabinoids $(31.0 \%)$, ethanol $(31.0 \%)$, levamisole $(31.0 \%)$, and free morphine $(31.0 \%)$. The average number of drugs/metabolites found on toxicology for fatalities with buprenorphine were 9.24 compared to 6.68 in those without buprenorphine. In one case, buprenorphine was the only drug listed to cause death; all other fatalities with buprenorphine on toxicology reported additional drugs contributing to death. Naloxone was not detected in any fatalities with buprenorphine on toxicology.

Conclusion: The majority of overdose cases with buprenorphine on toxicology had fentanyl present. More coingestant drugs and metabolites were found in fatal overdose cases with buprenorphine compared to fatal 
overdose cases without buprenorphine on toxicology. Further work should explore the use of postmortem concentrations of buprenorphine, norbuprenorphine, and other opioid metabolites to determine the role of buprenorphine in fatal overdose pharmacology.

\section{Practice Patterns of Emergency Department Physicians Administering Naloxone for Patients with Suspected Opioid Overdose}

Michael A Blaszak ${ }^{1}$, Stephanie N Chilton ${ }^{1}$, Stefan Knezevic ${ }^{1}$, Justin W Yan $^{1}$, Kristine P Van Aarsen ${ }^{2}$, Sarah Detombe ${ }^{3}$, Morgan AA Riggan ${ }^{1,3}$ ${ }^{1}$ Schulich School of Medicine and Dentistry, Western University, London, ON, Canada. ${ }^{2}$ London Health Sciences Centre, London, ON, Canada. ${ }^{3}$ Poison and Drug Information Service, Alberta Health Services, Calgary, $A B$, Canada

Background: Naloxone is recommended for reversing opioid-associated respiratory depression. There is wide variability in emergency department (ED) practice patterns regarding naloxone use, dosing, and observation time post-administration.

Objective: Describe the naloxone practice patterns of ED physicians managing suspected opioid overdose patients.

Methods: This is a single center retrospective chart review of 113 patients ( $\geq 18$ years) presenting with suspected opioid overdose who were administered naloxone in the ED (January 1-December 31, 2017). This study was performed at an academic tertiary care center consisting of two EDs with a combined annual census of 150,000 visits. Patients were identified electronically to collect demographics, naloxone dosage and infusion initiation, and disposition data. Clinical information regarding apparent indications for naloxone administration, response to therapy, and adverse effects were abstracted. Variability in administration was quantified via the initial and total dose administered. Data were analyzed using standard descriptive statistics.

Results: Indications for naloxone administration were as follows: level of consciousness $(50.5 \%)$, respiratory depression $(4.0 \%)$, miosis $(1.0 \%)$, a combination of these factors $(19.8 \%)$, or undocumented (24.8\%). Median initial dose was $0.40 \mathrm{mg}$ (IQR 0.20 $0.40 \mathrm{mg}$ ). Median total naloxone administered in the ED was $0.48 \mathrm{mg}$ (IQR $0.35-1.2 \mathrm{mg}$ ). The initial dose resulted in a response in $43.1 \%$, with $36.0 \%$ of responders later experiencing subsequent respiratory depression. Thirty-one percent received a naloxone infusion. Four patients experienced emesis following naloxone. Median length of ED stay was 7.0 hours (IQR 4.09.5 hours), and median hospital admission was 3.0 days (IQR 1.0-5.0 days). Median observation time prior to discharge was 4.0 hours (IQR 2.0-8.0 hours). Ultimate disposition home, the ward, or the intensive care unit was $47.1,42.2$, and $9.8 \%$ respectively (1.0\% deceased).

Conclusion: The dose and usage of naloxone by ED physicians in this study is variable. Further prospective studies are needed to determine the effective naloxone dosing strategy.

\section{Day 2: Posters, Abstracts 069-116}

069. The Etiology of Mesoamerican Nephropathy: Environmental Vs. Toxic Vs. Infectious?

James H. Diaz

LSU Health Sciences Center, New Orleans, LA, USA

Background: Mesoamerican nephropathy $(\mathrm{MeN})$ is an epidemic form of chronic kidney disease of unknown etiology ( $\mathrm{CKDu}$ ) confined to the Pacific lowlands of Central America that afflicts young male sugarcane and mining workers in areas without access to renal replacement therapies. Unrelated to common causes of CKD, such as diabetes and hypertension, MeN has several other regional risk factors.
Hypothesis: Can evidence-based epidemiological investigations identify and stratify the most significant risk factors for $\mathrm{MeN}$ in order to support epidemic-control interventions?

Methods: This is a convenience sample survey that queried Internet search engines with key words (MeN, $\mathrm{CKDu}$ ) in order to identify casecontrol and cohort studies of MeN during the study period, 1990-present. Results: Environmental risk factors for $\mathrm{MeN}$ included strenuous labor in high temperatures with inadequate rehydration. Toxic risk factors included agrochemical exposures, arsenic and cadmium in drinking water, highpurine-containing diets causing uricosuria, and abuse of NSAIDs and herbal pain remedies containing aristolochic acid for work-related musculoskeletal pain. Infectious risk factors included rodent-borne leptospirosis and Hanta virus infections causing renal syndromes. Case-control studies that matched male coffee workers in the Central American highlands as controls with MeN cases in the lowlands did not detect $\mathrm{MeN}$ in controls. Both groups had similar agrochemical exposures, diets, and analgesic use eliminating toxic exposures as risk factors. In addition, another case-control study in a Nicaraguan mining community found no causal links between leptospirosis and Hanta virus seroprevalence and MeN. Strenuous labor in high temperatures and inadequate hydration were the most significant risk factors for MeN.

Conclusion: MeN-like clusters of $\mathrm{CKDu}$ will continue to occur among outdoor laborers throughout the tropics until agricultural and mining industries in developing nations are completely mechanized. Only occupational heat stress monitoring, evaluation, and prevention programs can curtail the current epidemic of $\mathrm{MeN}$ in young male workers in Central America.

070. Working Toward Gender Equality: the Status of Women in Toxicology at ACMT and AACT

Meghan B Spyres ${ }^{1}$, Jennie A Buchanan ${ }^{2}$, Elizabeth C Moore ${ }^{1}$, AnneMichelle Ruha ${ }^{3}$, Ayrn D O'Connor ${ }^{3}$

${ }^{1}$ University of Southern California, Los Angeles, CA, USA. ${ }^{2}$ University of Colorado, Denver, CO, USA. ${ }^{3}$ Banner University Medical Center Phoenix, Phoenix, AZ, USA

Background: Women are underrepresented in leadership positions in academic medicine. The status of women in toxicology, with regard to leadership and supporting activities at ACMT and AACT, is unknown.

Hypothesis: Women in toxicology are underrepresented in leadership at ACMT and AACT.

Methods: ACMT and AACT data were queried from internal and external sources for membership data, leadership positions, grants, awards, and speakers. Data through 2019 were included along with retrievable historic data. Research presentations and student and travel awards were excluded.

Results: ACMT-In 2019, 246 (31\%) women are ACMT members. Twelve (25\%) women have served as ACMT board members, and four (36\%) have been president. In 2019, women comprise $39 \%$ of ACMT board members. Since ACMT's formation, one (4\%) woman has received the organization's highest award, the Ellenhorn award; six (21\%) women have received ACMT's other awards. Fourteen (67\%) have received ACMT grants. Since 2015, 34\% of speakers at the Annual Scientific Meeting (ASM) have been women. Women comprised $28 \%$ of panelists and $40 \%$ of invited lecturers at ASM since 2015; in 2018, there were no women panelists. AACT - In 2019, 225 (35\%) women are members of AACT. Two (8\%) women have served as AACT president. Twenty-seven Trustees $(51 \%)$ have been women. Three $(15 \%)$ women have received the prestigious AACT Career Achievement Award. Women received 24\% of AACT's remaining awards. Since 2015, two (67\%) women have received AACT grants. Since $2015,46 \%$ of speakers at NACCT have been women, including $41 \%$ of panelists and $45 \%$ of invited lecturers.

Conclusion: Women comprise approximately one third of both professional toxicology organizations. The proportion of women in leadership is low but reflective of membership. In both organizations, women 
receive fewer awards yet more grants than expected. Women are proportionally represented as speakers at national toxicology conferences.

071. Second Time's the Charm: Methotrexate Toxicity in a Peritoneal Dialysis Patient After Two Low Oral Doses

Rachel M Shively ${ }^{1,2}$, Joshua N Nogar ${ }^{1}$

${ }^{1}$ Northwell Health, Manhasset, NY, USA. ${ }^{2}$ Elmhurst Hospital-Mount Sinai Health System, New York, NY, USA

Background: Methotrexate (MTX) is first-line therapy for rheumatoid arthritis (RA) as per most recent consensus rheumatology guidelines. While the antimetabolite has proved very effective, even low-dose MTX has caused life-threatening pancytopenia in end-stage renal disease (ESRD) patients. This risk is potentially greater in patients receiving peritoneal dialysis (PD).

Methods: We report a case of pancytopenia requiring filgrastim treatment in an ESRD patient on PD after receiving two oral doses of $7.5 \mathrm{mg}$ MTX. Results: A 77-year-old man with ESRD requiring daily PD began $7.5 \mathrm{mg}$ weekly regimen of MTX for newly diagnosed rheumatoid arthritis. Five days after receiving his second dose of MTX, he developed stomatitis. Upon evaluation 2 days later, he was found to have pancytopenia with white blood cells $0.5 \times 10^{9} / \mathrm{L}$, hemoglobin $9.5 \mathrm{~g} / \mathrm{dL}$, and platelets $11 \times$ $10^{9} / \mathrm{L}$. He also had mild hepatotoxicity with AST $49 \mathrm{U} / \mathrm{L}$ and ALT $78 \mathrm{U} /$ L. His serum methotrexate concentration 7 days post-MTX was < $0.04 \mathrm{mcmol} / \mathrm{L}$ (toxic threshold $>1 \mathrm{mcmol} / \mathrm{L}$ ). He was admitted to the hospital and treated with $100 \mathrm{mg} / \mathrm{m}^{2}$ leucovorin IV q6h for 2 weeks. He also received $480 \mathrm{mcg}$ SQ filgrastim daily for 6 days and transfusion of $1 \mathrm{U}$ pooled platelets and $1 \mathrm{U}$ pRBCs. His oral lesions resolved after 6 days; blood counts normalized after 7 days. He continued to receive daily peritoneal dialysis throughout hospitalization.

Conclusion: MTX is primarily renally eliminated and accumulates in third space fluids. Because of PD patients' renal function and iatrogenic ascites, MTX toxicity can be protracted and significant, even after low oral doses. Although some pharmaceutical guides advise $50 \%$ reduction of MTX dose post-dialysis, consensus rheumatology guidelines have not focused on its risks in ESRD patients, and use continues. For this reason, restriction in ESRD patients should be recommended in consensus guidelines, stressing MTX use only in patients with no alternative therapy.

072. Epidemiology and Treatment of Z-Drug Toxicity as Reported by Medical Toxicologists: a ToxIC Database Study

Richard J Chen ${ }^{1}$, Muhammed Ershad ${ }^{1}$, Sharan Campleman ${ }^{2}$, David Vearrier $^{3}$, On Behalf of the ToxIC Investigators Consortium (ToxIC) ${ }^{I}$ Einstein Healthcare Network, Philadelphia, PA, USA. ${ }^{2}$ American College of Medical Toxicology, Phoenix, AZ, USA. ${ }^{3}$ University of Mississippi Medical Center, Jackson, MS, USA

Background: Z-drugs (eszopiclone, zolpidem, zopiclone, and zaleplon) are a class of GABAergic medications that are commonly prescribed as alternatives to benzodiazepines for the treatment of insomnia. Postmarketing experience with these medications has demonstrated that they have adverse effect profile and addictive potential similar to benzodiazepines.

Research Question: What is the epidemiology, signs/symptoms, and treatment of Z-drug toxicity as reported by the ToxIC Registry?

Methods: We performed a retrospective analysis of all cases of Z-drug toxicity reported to the ToxIC Registry from 2010 through 2018, inclusively. Results: A total of 886 cases involving Z-drugs were reported to the ToxIC Registry from 2010 through 2018. Zolpidem was the most common exposure, involving $809(91 \%)$ of all cases. There was no trend in the relative frequency of individual Z-drug mentions over the study period $(p=$ NS). Patients aged 19-65 years constituted $82 \%$ of cases with the second most common age range being $13-18$ years $(8 \%)$. Females comprised the majority of cases overall $(n=561,63 \%)$ and for each adult age group. Most cases (92\%) of toxicity were due to intentional ingestion, either due to misuse/abuse or attempted self-harm. The most common adverse effect was CNS depression (71\%) followed by respiratory depression $(17 \%)$ and delirium (12\%). The most common treatment modalities were IV fluid resuscitation (27\%) followed by endotracheal intubation (15\%). Most cases $(78 \%)$ involved coingestants, most commonly non-z-drug sedativehypnotics (56\%) followed by antidepressants (38\%). No deaths were reported following isolated exposure to Z-drugs. However, there were three deaths reported among patients with multiple ingestions.

Conclusion: Z-drug toxicity most commonly occurs due to intentional overdose with clinical effects similar to benzodiazepine toxicity. Treatment is primarily supportive in nature. This data suggests that Zdrug overdoses have low mortality with significant clinical effects resulting only in combination with other xenobiotics.

\section{ToxIC \\ This research was performed in collaboration with the ACMT Toxicology Investigators Consortium.}

\section{Treatment of Antimuscarinic Delirium with Rivastigmine as an} Alternative to Physostigmine

Adrienne R Hughes ${ }^{1,2}$, Kerry K Moore ${ }^{1}$, Nathan D Mah ${ }^{1}$, Asha R Birmingham ${ }^{1}$, Roger K Clark ${ }^{1}$, John Thompson ${ }^{1}$, Robert G Hendrickson $^{1,2}$

${ }^{I}$ Oregon Health and Science University, Portland, OR, USA. ${ }^{2}$ Oregon Poison Center, Portland, OR, USA

Background: Rivastigmine is a long-acting cholinesterase inhibitor approved for the treatment of Alzheimer's disease and other neurodegenerative conditions. Little evidence exists to suggest efficacy of longer-acting cholinesterase inhibitors in treating symptoms of antimuscarinic delirium. We present a case of antimuscarinic delirium successfully treated with rivastigmine.

Methods: A 36-year-old woman was brought to our emergency department (ED) after being found unresponsive. She was covered in pinktinged emesis next to an empty bottle of diphenhydramine. Initial vital signs were within normal limits. The patient was agitated, restless, confused, and mumbling incoherently. She had minimally responsive mydriatic pupils, horizontal nystagmus, and dry skin and mucous membranes. The patient developed tachycardia and became agitated (RASS +3 ). She was administered $1 \mathrm{mg}$ lorazepam intramuscularly followed by $1 \mathrm{mg}$ lorazepam intravenously with minimal improvement. Physostigmine was on national shortage and toxicology recommended rivastigmine as an alternative therapy. The patient was administered rivastigmine $3 \mathrm{mg}$ $\mathrm{PO}$, followed by two additional $3 \mathrm{mg}$ doses over the course of 2 hours. On re-evaluation, the patient remained slightly confused, but showed improvement in her agitation (RASS +1). Over the following day, the patient received an additional $3 \mathrm{mg}$ of rivastigmine with complete resolution of her symptoms. She developed no adverse effects or recurrent symptoms of antimuscarinic toxicity and was discharged.

Discussion: Rivastigmine, an orally administered and longer-acting acetylcholinesterase inhibitor, was used as an alternative to physostigmine, producing effective and long-lasting symptom reversal. Agents such as rivastigmine have potential therapeutic advantages due to their slower rate of CNS penetration, longer duration of action, and oral and transdermal administration routes. Longer-acting reversal agents may be preferable when prolonged delirium is expected.

Conclusion: Rivastigmine, with its oral administration route and favorable kinetics, may be a useful alternative treatment option for antimuscarinic delirium.

074. Elevated Methemoglobin Levels in Patients Treated with Hydroxocobalamin After Suspected Cyanide Exposure: a Case 
Series

Emily Kiernan $^{1,2}$, Joseph E Carpenter ${ }^{1,2}$, Alaina Steck ${ }^{1,2}$, Brent Morgan $^{1,2}$, Brian Patrick Murray ${ }^{1,2}$

${ }^{1}$ Emory University School of Medicine, Atlanta, GA, USA. ${ }^{2}$ Georgia Poison Control Center, Atlanta, GA, USA

Background: Cyanide $\left(\mathrm{CN}^{-}\right)$toxicity most commonly occurs during enclosed-space fires. Historically, the first step in treating $\mathrm{CN}^{-}$toxicity utilized antidotes to induce methemoglobinemia (metHb). This is concerning in patients who are already hypoxemic or have elevated carboxyhemoglobin levels. Hydroxocobalamin (OHCob) is now the first-line antidote for $\mathrm{CN}^{-}$toxicity as it does not induce metHb.

Hypothesis: We observed elevated metHb levels in several patients treated with $\mathrm{OHCob}$ for possible $\mathrm{CN}^{-}$toxicity and developed this study to determine the prevalence of metHb formation following administration of $\mathrm{OHCob}$.

Methods: This is a single-center, retrospective case series including patients who received 5 or $10 \mathrm{~g}$ of hydroxocobalamin from January 01,2011 , through April 30, 2019. Data was analyzed using descriptive statistics.

Results: A total of 27 cases of $\mathrm{OHCob}$ administration were identified over the study period. The median age was 53 years (IQR 38-64) and 20 $(74.1 \%)$ were male. Exposure to a house fire or smoke inhalation was the reason for $\mathrm{OHCob}$ administration in $21(77.8 \%)$ of the patients, while two $(7.4 \%)$ were for severe undifferentiated acidosis and three $(11.1 \%)$ were for another reason. Of patients who presented from a fire, the median total body surface area burned was $11 \%$ (range $0-95 \%$ ). Five (18.5\%) patients received $10 \mathrm{~g}$ of hydroxocobalamin, while the rest received $5 \mathrm{~g}$. Six $(22.2 \%)$ of the patients developed methemoglobinemia, all occurring after administration of hydroxocobalamin. The median peak level was $6.3 \%$ (range $2.0-16.3 \%$ ) at a median time of 11.4 hours post-administration. Two patients received methylene blue and neither responded to the antidote. Death occurred in $17(63 \%)$ of the cases.

Conclusion: We found a noteworthy prevalence of methemoglobinemia that consistently developed after the administration of hydroxocobalamin. This could have profound implications for patients who are already hypoxemic or have impaired oxygen carrying capacity secondary to carboxyhemoglobin.

075. Fomepizole as an Adjunctive Treatment in Severe Acetaminophen Ingestions

Kartik R Shah ${ }^{1}$, Carolyn Fox ${ }^{1}$, Ann-Jeannette Geib ${ }^{1}$, Christine Murphy ${ }^{1}$, Kathryn T Kopec ${ }^{1}$, Anna R Dulaney ${ }^{2}$, Michael C Beuhler ${ }^{2}$

${ }^{1}$ Atrium Health's Carolinas Medical Center, Charlotte, NC, USA. ${ }^{2}$ North Carolina Poison Control, Charlotte, NC, USA

Background: $N$-acetylcysteine (NAC) is the mainstay treatment for acetaminophen (APAP) toxicity; however, some patients suffer severe morbidity and mortality despite NAC. Fomepizole has molecular, biochemical, animal, and human data suggesting it prevents acetaminophen toxicity by inhibiting NAPQI formation and mitochondrial collapse.

Hypothesis: Fomepizole use in severe acetaminophen toxicity is safe and beneficial.

Methods: This is a retrospective case series of three acetaminophen toxic patients with concern for significant morbidity and possible mortality. They were initially screened with a multiplication product (APAP $\times$ aminotransferase $(\mathrm{AT}))>10,000 \mathrm{mcg} / \mathrm{mL} \mathrm{IU} / \mathrm{L}$. Every patient manifested SIRS, lactic acidosis, or arterial acidemia; each has been correlated to death without liver transplantation. All were treated with fomepizole early in their care and NAC. None had renal replacement therapy.

Results: Case 1: 62-year-old female with acute ingestion. Estimated 5 hours APAP, $1014 \mathrm{mcg} / \mathrm{mL}$; ALT, $10 \mathrm{IU} / \mathrm{L}$; AST, $13 \mathrm{IU} / \mathrm{L}$. Eight hours later, $\mathrm{APAP} \times \mathrm{AT}=521,202 \mathrm{mcg} / \mathrm{mL} \mathrm{IU} / \mathrm{L}$. Fifteen hours later, heart rate was $99 \mathrm{bpm}$ and respiratory rate was 28 breaths/minute. Case 2: 56-yearold female with acute on chronic ingestion. Initial APAP, $298 \mathrm{mcg} / \mathrm{mL}$;
ALT, $326 \mathrm{IU} / \mathrm{L} ;$ AST, $245 \mathrm{IU} / \mathrm{L}$. APAP $\times$ AT = 97,148 mcg $/ \mathrm{mL} \mathrm{IU} / \mathrm{L}$. After $40 \mathrm{cc} / \mathrm{kg}$ IV crystalloid, lactate was $9.3 \mathrm{mmol} / \mathrm{L}$ and arterial $\mathrm{pH}$ was 6.9. Case 3: 58-year-old female with unknown time of acute ingestion. Initial APAP, $22.4 \mathrm{mcg} / \mathrm{mL}$; ALT, $3246 \mathrm{IU} / \mathrm{L}$; AST, $5971 \mathrm{IU} / \mathrm{L}$. $\mathrm{APAP} \times \mathrm{AT}=133,750 \mathrm{mcg} / \mathrm{mL}$ IU/L. After $4 \mathrm{~L}$ IV crystalloid, lactate was $4.0 \mathrm{mmol} / \mathrm{L}$. Peak AT was 3466-11,001 IU/L; INR, 3.6-5.8. All survived to hospital discharge without liver transplantation.

Conclusion: Fomepizole may be a useful adjunct to NAC in severe APAP toxicity where biomarkers suggest increased risk of death.

\section{High-Dose Physostigmine After a Large Atropine Overdose}

\section{Mark Huang, Joshua N Nogar, Vincent Lee, Wells Brambl Northwell, Manhassett, NY, USA}

Background: Physostigmine is commonly administered diagnostically in small $(2 \mathrm{mg})$ doses to reverse the symptoms of anticholinergic toxicity. However, it is not typically administered in large $(10 \mathrm{mg})$ doses to reverse antimuscarinic tachycardia.

Research Question: Can physostigmine be used to mitigate prolonged antimuscarinic tachycardia in a patient with baseline poor baseline cardiac function?

Methods: An 82-year-old female with end-stage cardiomyopathy ( $\mathrm{EF}<$ $25 \%$ ), atrial fibrillation, and rheumatoid arthritis presented from home hospice with profound anticholinergic toxicity after accidentally receiving $4 \mathrm{~mL}$ of $1 \%$ atropine solution, instead of four drops. Upon arrival to the ED, her vital signs were HR $140 \mathrm{bpm}$, BP 112/55 mmHg, temp $36.5^{\circ} \mathrm{C}, \mathrm{RR} 20, \mathrm{SaO}_{2} 100 \%$ on room air. Physical exam was notable for dry mucous membranes, $8 \mathrm{~mm}$ pupils, tachycardia, and absent bowel sounds. Given her fragile cardiac status, physostigmine was administered, $1 \mathrm{mg}$ at a time, with the endpoint goal of controlling her heart rate. Ten $1 \mathrm{mg}$ doses were administered every 10 minutes. After $5 \mathrm{mg}$, she responded appropriately to questions by shaking her head in a yes/no manner. After $8 \mathrm{mg}$, she could state her name and respond with yes or no answers. After $9 \mathrm{mg}$, she could speak and hold a conversation. After $10 \mathrm{mg}$, her heart rate decreased from $140 \mathrm{bpm}$ to $113 \mathrm{bpm}$, and lacrimation was present. The next day, her HR was $105 \mathrm{bpm}$, and she was discharged to the hospice facility.

Results: Anticholinergic toxicity is typically not life-threatening. In this instance, physostigmine was used out of concern for impending cardiac decompensation. There is one prior case in the literature of an atropine overdose requiring $14 \mathrm{mg}$ of physostigmine to avoid intubation.

Conclusion: Large atropine overdoses are rare, but can cause significant morbidity in susceptible patients. High-dose physostigmine may be used to alleviate antimuscarinic tachycardia in the right clinical scenario.

077. Nebulized Naloxone in Suspected Opioid Overdose Patients at an Academic Medical Center Emergency Department

Kaleb Enlow ${ }^{1}$, Regan Baum ${ }^{2}$, Abby Bailey ${ }^{2}$, Peter Akpunonu ${ }^{3,4}$

${ }^{1}$ University of Kentucky College of Pharmacy, Lexington, KY, USA.

${ }^{2}$ University of Kentucky Healthcare, Lexington, KY, USA. ${ }^{3}$ University of Kentucky, Lexington, KY, USA. ${ }^{4}$ Kentucky Poison Control Center, Louisville, KY, USA

Background: Naloxone is a competitive opioid receptor antagonist used to reverse the effects of opioids. While typically administered intravenously, administration of naloxone via nebulization may have advantages: does not require intravenous access, dose can be more readily adjusted as compared to the intranasal commercially available product, provides a steady low dose similar to an intravenous infusion, and is selftitrating. Despite multiple blog posts advocating for its use, there is minimal published data regarding nebulized naloxone (NN). The purpose of this study was to characterize the clinical situations in which NN was used, and record any outcomes associated with its administration. 
Methods: This retrospective study utilized a chart review to characterize the usage of $\mathrm{NN}$ at an academic medical center. Patients who received NN for suspected opioid overdose from EMS or in the emergency department at the University of Kentucky between 2017 and 2019 were included in this study. Patients who received other formulations of naloxone in addition to $\mathrm{NN}$ were included. $\mathrm{NN}$ is prepared by placing $2 \mathrm{mg}$ of naloxone into the nebulizer with an additional $3 \mathrm{ml}$ of normal saline. Dose was administered until the patient spontaneously removes non-rebreather mask.

Results: Eleven patients were identified as having received NN during the study period, with ages ranging from 25 to 53 years old. Response to $\mathrm{NN}$ was documented to be as fast as $5-10 \mathrm{~s} ; 60 \%$ of patients received multiple formulations of naloxone and one third of patients had urine drug screens positive for opioids. The average dose of naloxone administered was $5.2 \mathrm{mg}$ with no adverse reactions documented; $20 \%$ left AMA. Conclusion: This is a small case series of patients treated for suspected opioid overdose with $\mathrm{NN}$. NN is a viable alternative route of treatment for opioid overdose. It has multiple benefits including self-titration and decreased incidence of induction of opioid withdrawal.

\section{Iatrogenic Calcium Overdose: a Unique Acid-Base Disturbance}

S J Weiss, S J Walsh, S E Simpson

Albert Einstein Healthcare Network, Philadelphia, PA, USA

Background: Calcium is often included in the treatment for calcium channel blocker overdose. Calcium toxicity-associated lactic acidosis has not been reported.

Hypothesis: Stewart acid-base analysis explains elevated lactate in this case of acute iatrogenic hypercalcemia.

Methods: This is a case report of calcium infusion following amlodipine ingestion. A 19-year-old woman presented to the ED oriented, anxious, tachycardic, and tachypneic 45 minutes after intentional amlodipine overdose. Initial BP was 122/75 mmHg. Activated charcoal was administered. Poison control recommended monitoring and, if decompensation occurred, infusion of crystalloid, calcium gluconate (CG) $0.05 \mathrm{~g} / \mathrm{kg} / \mathrm{hour}$, and high-dose insulin. Systolic BP ranged between 90 and $110 \mathrm{mmHg}$; because of decrease from presentation, crystalloid and CG infusions were initiated. Abdominal pain and vomiting developed 6 hours after presentation (total CG $10.5 \mathrm{~g}$ ). There was no hemodynamic change, but infusions were continued. The patient received $5.6 \mathrm{~L}$ normal saline, $1.5 \mathrm{~L}$ lactated ringers, and $30 \mathrm{~g} \mathrm{CG}$ total over 12 hours, when infusions were stopped.

Results: Her calcium level peaked at $21.1 \mathrm{mg} / \mathrm{dL}$. She developed nonanion gap metabolic acidosis (NAGMA), with chloride $115 \mathrm{mmol} / \mathrm{L}$ and bicarbonate $14 \mathrm{mmol} / \mathrm{L}$. Lactate peaked at $8.27 \mathrm{mmol} / \mathrm{L}$ (Fig. 1). Strong ion gap was -13 , accounted for by hypercalcemia (Fig. 2). Calcium level declined to $12.7 \mathrm{mg} / \mathrm{dL}$ over 13 hours and other laboratory abnormalities normalized. She was discharged without further complications on day 2. Nineteen-hours post-ingestion, amlodipine level was $170 \mathrm{ng} / \mathrm{mL}$ (therapeutic range 2-25). In this case, hyperchloremia from crystalloid infusion caused NAGMA and hypobicarbonatemia, with inability to balance cations from iatrogenic hypercalcemia. Lactate increase may have been a secondary compensation for charge balance. Her supratherapeutic amlodipine level without hemodynamic instability supports literature that serum concentration does not predict toxicity.

Conclusion: This case of iatrogenic hypercalcemia uniquely included combined hyperchloremic/lactic NAGMA, but a negative strong ion gap.

\section{Severe Colchicine Toxicity Treated with Red Cell Exchange}

Alexander M Sidlak, Shirley Shao, Andrew F Micciche, Rachael C Westover, Ian M Harrold, Anthony F Pizon

School of Medicine, University of Pittsburgh, Pittsburgh, PA, USA

Background: Colchicine toxicity can progress to multi-organ failure and carries a high mortality. Treatment options are limited.
Hypothesis: Red cell exchange (RCE) may be an effective treatment in colchicine toxicity.

Methods: This is a single patient case report. RCE was performed after the onset of multi-organ failure. Automated RCE exchanges a patient's autologous red blood cells (RBCs) for packed RBCs from the blood bank (10 units in this case). This patient's total blood volume was processed 1.5 times to remove approximately $70 \%$ of their own RBCs.

Results: A 33-year-old woman developed worsening vomiting and diarrhea 36 hours after a hospitalization for pericarditis. She arrived hypotensive (SBP $66 \mathrm{mmHg}$ ) and was treated for presumed septic and cardiogenic shock. On HD 1, she developed anuric renal failure requiring CRRT and hepatic necrosis (peak AST $4500 \mathrm{IU} / \mathrm{L}$ ). She had worsening shock, requiring escalating doses of vasopressors (epinephrine, norepinephrine, vasopressin, and milrinone) and lactate continued to rise (peak $12 \mathrm{mMol} /$ L). She progressed to ARDS and she was paralyzed. Echocardiogram showed severe global hypokinesis (EF of 15\%) and her troponin peaked at $73.14 \mathrm{ng} / \mathrm{mL}$. With colchicine toxicity suspected, RCE was initiated. After transfusion, her vasopressor requirements improved, as did her cardiac and hepatic function. She developed late neutropenia (ANC of $200 / \mathrm{mm}^{3}$ ) on HD 6. She was successfully extubated on HD 13 and admitted to taking her entire prescription of colchicine (sixty $0.6 \mathrm{mg}$ tabs). Conclusion: Serum and whole blood colchicine levels prior to RCE were 5.7 and $17 \mathrm{ng} / \mathrm{mL}$. Post-exchange, whole blood level was $7.6 \mathrm{ng} / \mathrm{mL}$. RCE reduced whole blood levels in our anuric patient, while plasma levels remained unchanged (5.7 to $5.3 \mathrm{ng} / \mathrm{mL}$ ). Despite development of multi-organ dysfunction, which is associated with a very high mortality, this patient survived with supportive care and RCE.

\section{Treatment of Acute Methadone Overdose with Buprenorphine Induction}

Alexander M Sidlak ${ }^{1}$, Julie B McCausland ${ }^{1}$, Ryan T Marino ${ }^{2}$

${ }^{1}$ University of Pittsburgh School of Medicine, Pittsburgh, PA, USA. ${ }^{2}$ Case Western Reserve School of Medicine, Cleveland, $\mathrm{OH}$, USA

Background: Methadone overdose causes prolonged duration of toxicity, often requiring continuous naloxone infusion and intensive care monitoring. Recent case reports have shown that intravenous buprenorphine is safe and effective in antagonizing mu opioid receptor effect in methadone overdoses; however, these isolated cases have used low-dose buprenorphine and still required subsequent naloxone infusion. Furthermore, patients using methadone nonmedically can benefit from medical treatment for opioid use disorder.

Hypothesis: Sublingual buprenorphine may obviate the need for naloxone infusions after acute overdose of long-acting opioids.

Methods: This is a single patient case report. A 53-year-old woman presented after overdose on $110 \mathrm{mg}$ of liquid methadone she had purchased for recreational use. She had a history of opioid use disorder and had been in recovery without medications for 6 years. On presentation, she was initially comatose with respiratory depression requiring naloxone. Over 12 hours, she had recurrent bradypnea and hypoxemia, requiring repeated dosing of naloxone. Two hours after her third dose of naloxone, she had recurrent somnolence and bradypnea, and the decision was made to administer buprenorphine-naloxone, 8-2 mg sublingually. Results: She was admitted with persistent symptoms of drowsiness for the next 24 hours but did not have hypoxemia or bradypnea and did not require additional naloxone. Buprenorphine was continued for opioid use disorder after resolution of acute toxicity.

Conclusion: Buprenorphine has mu opioid receptor agonist effects and a significantly longer half-life than naloxone. In this case, these effects prevented recurrent toxicity. While a potential for precipitated withdrawal exists in chronic opioid use given buprenorphine's high affinity for the $\mathrm{mu}$ receptor and relatively long duration of action, sublingual buprenorphine may be effective as antidotal therapy for acute overdose of long-acting opioids. In acute overdose, buprenorphine may be effective 
in obviating the need for intensive care treatment and monitoring.

\section{Utilizing Transdermal Buprenorphine for Rapid Induction of Opioid Agonist Therapy (OAT) in Hospitalized Patients on Either Short- or Long-Acting Opioids}

Andrew F Micciche, Rachael C Westover, Alexander M Sidlak, Shirley Shao, Michael G Abesamis

School of Medicine, University of Pittsburgh, Pittsburgh, PA, USA

Background: Buprenorphine is a common pharmaceutical agent employed as opioid agonist therapy (OAT) for the treatment of opioid use disorder. While there are many advantages to its use over methadone, buprenorphine's action as a partial agonist at the mu opioid receptor, as well as its higher binding affinity, can result in precipitated opioid withdrawal in patients on concurrent short- or long-acting opioids.

Hypothesis: Transdermal buprenorphine can be utilized for expedited transition to buprenorphine in patients in whom it would traditionally be contraindicated.

Methods: Our toxicology service has developed a guideline for transitioning to buprenorphine from full opioid agonists. A $20-\mathrm{mcg}$ transdermal buprenorphine patch is applied while patient is concomitantly taking full opioid agonists. An additional 20-mcg patch is applied in patients with perceived higher opioid requirements. Transdermal buprenorphine administration continues for 12-24 hours, at which time patients are initiated on sublingual buprenorphine-naloxone, using $2-0.5 \mathrm{mg}$ dosing as needed every 2 hours for a total of four doses. Full opioid agonists are discontinued after induction, and patch is removed.

Results: We describe a case series of five patients treated successfully with the above guideline. All patients had recent or concomitant use of full opioid agonists at time of initiation and suffered no complications. This is exemplified in our series by a 27 -yearold male who was on $95 \mathrm{mg}$ of methadone daily, with last use the day prior to presentation. Transition to buprenorphine was desired due to a significantly prolonged QTc interval. Transdermal buprenorphine was initiated on hospital day 2, and the patient was given sublingual buprenorphine-naloxone without incident on hospital day 3.

Conclusion: Transdermal buprenorphine can be used for rapid induction of OAT in hospitalized patients on full opioid agonists.

\section{A Review of the Existing Literature on Buprenorphine Pharmacogenomics}

Christopher Meaden, Cynthia Santos, Diane Calello, Lewis Nelson Rutgers New Jersey Medical School, Newark, NJ, USA

Background: Buprenorphine is an effective treatment for opioid dependence although it demonstrates individual variability in efficacy. Pharmacogenomics may explain this variability and could allow for individualized therapy. The Food and Drug Administration (FDA) and the Clinical Pharmacogenomics Implementation Consortium (CPIC) have guidelines on pharmacogenomic testing for some opioids (e.g., codeine); however, no guidelines exist for buprenorphine. Pharmacogenomic testing targets for buprenorphine include pharmacodynamic genes including the mu-opioid receptor (MOP receptor) and catechol-O-methyltransferase (COMT) and the pharmacokinetic genes such as the CYP enzymes. We attempted to identify genotypes associated with altered therapeutic dosing and increased rate of relapse in patients with opioid addiction receiving buprenorphine.

Methods: We searched PubMed using the keywords "buprenorphine" and "pharmacogenomics." All resulting studies were cataloged regarding their impact on dosing or clinical effectiveness.

Results: Fourteen clinical studies, 5 systematic reviews, 3 human tissue in vitro studies, and 1 case report were reviewed. Several genotypes and gene loci were identified. The OPRM1 A118G single nucleotide

polymorphism (SNP rs1799971) gene variant encoding the N40D MOP receptor was associated with variable efficacy and response to treatment in both adult and neonatal patients receiving buprenorphine for treatment of opioid withdrawal. An SNP associated with rs678849 of OPRD1, coding for the delta-opioid receptor, was associated with opioid relapse as indicated by opioid positive urine drug screens; there was also sexspecific SNP identified at rs581111 and rs529520 in the European American population. COMT variability, particularly in rs4680, was associated with length of hospital stay and need for treatment for neonatal abstinence syndrome. Variations of the pharmacokinetic gene for CYP3A4 showed that the ultrarapid metabolizer phenotype required higher doses of buprenorphine.

Conclusion: Based on very limited published data, there may be value to a better understanding of pharmacogenetic variability in treatment outcome with buprenorphine; however, further research on the pharmacogenomics of buprenorphine is needed.

083. Can a Brief Educational Intervention "Bup 101" Increase Emergency Department Initiation of Buprenorphine for Opioid Use Disorder?

Utsha G. Khatri ${ }^{1}$, Kathleen Lee ${ }^{2}$, Ted Lin ${ }^{2}$, Jeanmarie Perrone ${ }^{2}$

${ }^{1}$ University of Pennsylvania, Philadelphia, PA, USA. ${ }^{2}$ Perelman School of Medicine at the University of Pennsylvania, Philadelphia, PA, USA

Background: Despite the proven effectiveness of ED-initiated buprenorphine, many emergency medicine (EM) clinicians lack training on how to manage acute opioid withdrawal or initiate treatment with buprenorphine.

Methods: We conducted a pilot study to assess the feasibility and efficacy of an educational intervention ("Bup101") aimed at improving knowledge of and first time initiation of buprenorphine. We enrolled EM clinicians (EM residents, attendings, and advanced practice providers) who had not previously administered buprenorphine and provided a brief session on the fundamentals of opioid withdrawal and buprenorphine. We randomized clinicians to receive either the one-time training (standard arm, $n=24$ ) or the training plus weekly text delivered booster facts and a financial incentive to report first time administration of buprenorphine (enhanced arm, $n=25$ ). Our primary endpoint was the number of providers who reported administering buprenorphine in the 90 days following the intervention. Secondary endpoints were improvements in provider knowledge and changes in attitudes toward ED-initiated buprenorphine. Results: Preliminary results demonstrate no statistically significant differences between the two arms when comparing number of providers administering buprenorphine, changes in knowledge, or changes in attitudes toward buprenorphine in the ED. Approximately one third (31.5\%) of all enrolled providers in either arm self-reported administering buprenorphine within 90 days of the training. All providers' clinical knowledge improved by $19.6 \%(p=0.000)$ immediately after the training intervention. A smaller improvement of $9.8 \%(p=0.001)$ was sustained at 90 days. Results demonstrate more positive attitudes toward ED-initiated buprenorphine among all participants sustained at 90 days $(p=0.04)$.

Conclusion: A short educational intervention can lead to sustained improvements in ED providers' clinical knowledge of buprenorphine and lead to favorable attitudes toward adopting the practice; $31.5 \%$ of enrollees did administer buprenorphine following this brief intervention. Additional weekly reminders and a financial incentive did not confer added benefit to changing provider behavior, knowledge, or attitudes.

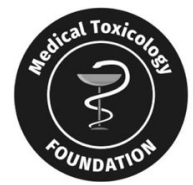

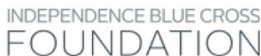

Independence

Medical Toxicology sorted by the Medical Toxicology Foundation through generous assistance from the Independence Blue Cross Foundation under the Supporting Treatment and Overdose Prevention (STOP) program. 
084. Using the Bernese Method to Transition a Patient from HighDose Methadone to Buprenorphine

Vincent S Ceretto, Michelle G Thompson, Kimberly Graeme, Michelle Ruha, Jennifer Mathis, Jerry Snow, Ayrn O'Connor

University of Arizona/Banner Health, Phoenix, AZ, USA

Background: Methadone is a common therapy for the treatment of opioid use disorder (OUD). Its use is sometimes limited by adverse effects, most notably, overdose and QT prolongation, which can cause torsades de pointes, a potentially life-threatening dysrhythmia. Consequently, clinicians may need to transition patients from methadone to buprenorphine, a therapy with a "ceiling effect" for respiratory depression and much less QT prolongation. However, this transition can be challenging because buprenorphine, a partial $\mu$-agonist, has stronger affinity than methadone for opioid receptors and can precipitate withdrawal. The Bernese method, whereby induction occurs gradually over a few days by using microdoses of buprenorphine and overlapping full opioid agonist therapy, has been recommended as an effective option with limited adverse effects.

Methods: This is a single case report. A 65-year-old male with hepatocellular carcinoma and OUD on $230 \mathrm{mg}$ daily methadone with QT prolongation $(524 \mathrm{~ms})$ experienced torsades de pointes that resolved with magnesium and defibrillation. The patient was decreased from 230 to $100 \mathrm{mg}$ methadone and concomitantly given microdoses of buprenorphine as follows: day 1 (0.3 $\mathrm{mg}$ IV BID), day 2 (0.6 mg IV BID), day 3 (0.8 mg IV BID), day 4 (1 mg SL BID), day 5 (2 mg SL BID), day 5 (4 mg SL BID), and day 6 (8 $\mathrm{mg}$ SL BID). On day 6, methadone was discontinued.

Results: The patient was successfully transitioned to buprenorphine over the course of 6 days without any signs of opioid withdrawal, discomfort, or repeat episodes of torsades de pointes. Repeat EKG on day 6 showed an improved QTcB of $382 \mathrm{~ms}$.

Conclusion: The Bernese method can be successfully used for buprenorphine induction when transitioning from high-dose methadone.

085. Treating Opioid Dependence and Pain in Hospitalized Patients Diagnosed with Endocarditis, Septic Arthritis, Epidural Abscess, or Osteomyelitis

Fabiola M Enriquez, Timothy J Wiegand, Kenneth Conner

University of Rochester, Rochester, NY, USA

Background: Hospital treatment of serious opioid-related infections (SORI), despite being associated with substantial morbidity and prolonged stay, often lacks treatment for dependence and addiction, which is often the underlying cause of the SORI. At our institution, the medical toxicology service performs consultations for SORI patients and is involved in medication initiation and adjustment, counseling and support, and assistance with "bridge" prescriptions to ongoing treatment after discharge.

Hypothesis: Buprenorphine is a major adjunct treatment in intravenous opioid abusers diagnosed with a SORI.

Methods: This is a retrospective review of toxicology billing data for five illustrative SORI cases representing IVDU-associated endocarditis, osteomyelitis, epidural abscess, and septic arthritis.

Results: All patients had prolonged hospital antibiotic courses: A 22year-old female IV heroin use and left clavicular osteomyelitis/septic arthritis (SC joint) started on buprenorphine-naloxone and bridged to inpatient treatment after 4-week hospitalization; a 50-year-old female IV heroin/cocaine diagnosed with epidural abscess and bacterial meningitis, reintroduced to buprenorphine-naloxone, and bridged to outpatient treatment after 4-week hospitalization; a 39-year-old female IV heroin/ cocaine with osteomyelitis (left foot/leg) started on buprenorphine-naloxone, weaned to IM-XR-naltrexone, during 4-week hospitalization, and discharged to PCP for monthly XR-naltrexone; a 25-year-old male IV heroin/cocaine with endocarditis/septic pulmonary emboli started on buprenorphine-naloxone, bridged to outpatient treatment after 5-week hospitalization; a 37-yearold female IV heroin/cocaine diagnosed with epidural abscess required surgical intervention after buprenorphine-naloxone initiated. Full agonists used perioperatively, transitioned to methadone and connected to opioid treatment program after 3-week hospitalization.

Conclusion: Appropriate care of patients with addiction-related SORIs includes initiating buprenorphine, methadone, or naltrexone during hospitalization; this sometimes requires nuanced pharmacologic management (e.g., buprenorphine combined with full-opioid agonists perioperatively). Supporting pharmacotherapies for dependence in SORI-related admissions represents an opportunity to expand medical toxicology practice.

086. Knowledge and Attitudes About Naltrexone Among Patients Presenting to the Emergency Department with Opioid-Related Complaints

Evan S Bradley, Brittany Chapman, Melissa Taylor, Jeffery Lai, Charlotte Goldfine, Kavita Babu

University of Massachusetts Medical School, Worcester, MA, USA

Background: Naltrexone is an important medication to assist in the treatment of opioid use disorder (OUD) in patients who are not interested in or are not good candidates for full agonist or partial agonist therapy. There remains persistent myths and disinformation about how the medication works and for whom it is a good treatment option. We sought to investigate the knowledge and attitudes about naltrexone in the community of patients with OUD served by our hospital and to assess perceived barriers to antagonist therapy.

Methods: We conducted semistructured interviews with a consecutive sample of 20 patients presenting to the UMass Memorial University Emergency Department with opioid-related complaints to assess their knowledge and attitudes about naltrexone. We also collected demographic data, opioid use history, and substance abuse treatment history.

Results: Participants ranged in age from 27 to 60 (mean age 38), 25\% were female, and $75 \%$ identified themselves as Caucasian. The majority (18/20) had public insurance (Medicare, Medicaid, or Mass Health). All participants had heard of naltrexone either through prior treatment programs for OUD or through acquaintances and knew it as its depot injection formulation, Vivitrol®. Most could identify it as an opioid antagonist although there was some confusion as to how treatment would affect them, with a number of participants expressing concern that they would experience withdrawal if they used opioids while on naltrexone. Although most individuals expressed interest in antagonist therapy, the most commonly identified barrier to treatment was the necessity of having to go through withdrawal prior to treatment initiation.

Conclusion: Our community of patients with OUD are aware of naltrexone, primarily in the form of depot injection and many expressed interest in antagonist treatment. The need to go through withdrawal and be opioid abstinent to receive treatment remains a significant barrier to antagonist treatment.

\section{Predictors of Serious Opioid-Related Adverse Drug Events (ORADE) in Hospitalized Patients}

Faisal S Minhaj ${ }^{1}$, Stephen H Rappaport ${ }^{2}$, Justin Foster ${ }^{2}$, Lauren Z Gashlin $^{2}$

${ }^{1}$ Maryland Poison Center, University of Maryland, Baltimore, MD, USA.

${ }^{2}$ University of Rochester Medical Center, Rochester, NY, USA

Background: Opioids are high-risk medications in the inpatient setting due to their potential for significant patient harm.

Research Question: The primary objective was to identify risk factors that predispose inpatients to develop opioid-related adverse drug events (ORADE) requiring the use of naloxone.

Methods: In a retrospective case-control study, patients were included according to the following criteria: $\geq 18$ years, $\geq 1$ administered opioid dose, and admitted for $\geq 24$ hours. Patients were excluded if they had a prehospital drug overdose, other indications for naloxone use, or were admitted to an 
ICU, psychiatric medical unit, or in the emergency department. Patients were classified as cases if naloxone was administered and a selection of controls was frequency matched 2:1 based on medical or surgical status. A logistic regression model was used to evaluate for risk factors for ORADE.

Results: A total of 275 cases and 592 control patients were included in the final analysis. In the case population, there were more patients $\geq 65$ years ( $47.3 \mathrm{vs}$ $27.4 \%, p<0.001$ ), fewer males (44 vs $52 \%, p=0.029$ ), a higher median serum creatinine (1.14 vs $0.83 \mathrm{mg} / \mathrm{dL}, p<0.001)$, and a prolonged length of stay (14 vs 11 days, $p<0.001)$. Variables that were associated with greater odds of naloxone administration included age $\geq 65$, female, length of stay, pulmonary diagnoses, use of gabapentinoids, and patient-controlled analgesia use. Antihistamines, continuous infusion, and intermittent nurse-administered intravenous bolus routes had a negative association with naloxone use.

Conclusion: Several risk factors were found to be associated with ORADE supporting many of the previously described risk factors and the discovery of potential new ones such as gabapentinoid use. Health care providers should consider the risk factors for hospitalized patients receiving opioids who may warrant lower doses, additional monitoring, or alternative agents.

088. Opioid Urine Drug Screening Capabilities of Hospital Emergency Departments in Delaware and Eastern Pennsylvania-2019

Haley O'Malley ${ }^{1}$, Anne Schneider ${ }^{2}$, Quan Nham ${ }^{3}$, Jeanette Trella ${ }^{4}$, Robb Bassett ${ }^{4}$, Kevin C. Osterhoudt ${ }^{4}$

${ }^{1}$ Ursinus College, Collegeville, PA, USA. ${ }^{2}$ Thomas Jefferson College of Pharmacy, Philadelphia, PA, USA. ${ }^{3}$ Wilkes University Nesbitt School of Pharmacy, Wilkes-Barre, PA, USA. ${ }^{4}$ The Poison Control Center at the Children's Hospital of Philadelphia, Philadelphia, PA, USA

Background: Opioid use disorder and opioid poisoning is a significant public health problem that burdens hospital emergency departments (EDs). Traditional immunoassay-based urine drug screens have good test performance characteristics in detecting heroin and morphine, but may be suboptimal at detecting semisynthetic opioids such as oxycodone, methadone, buprenorphine, or fentanyl.

Research Question: What are the capabilities of urine drug screening for opioids within DE and eastern PA?

Methods: Cross-sectional survey of hospital laboratories. The clinical chemistry laboratories from all acute care hospitals, within the designated coverage region of The Poison Control Center at the Children's Hospital of Philadelphia, were contacted by phone and queried about opioid detection capabilities of ED urine drug screens (UDS). Calls were made by three trained surveyors using a set script.

Results: Of the 77 eligible hospitals (68 in PA and 9 in DE), 70 (90.9\%) responded; 48/77 (62.3\%) confirmed that their onsite, real-time urine drug testing capabilities would be able to detect oxycodone, $43 / 77$ (55.8\%) methadone, $17 / 77$ (22.0\%) buprenorphine, and 21/77 (27.2\%) fentanyl. Only 14/ $77(18.2 \%)$ hospitals report being able to detect oxycodone, methadone, buprenorphine, and fentanyl within urine samples in real-time. Point-of-care drug screening, allowing rapid detection of a broad spectrum of opioids, has been adopted in 7/77 (9.1\%) hospital EDs. None of the hospitals in Delaware reported being able to detect all four opioids in their UDS.

Conclusion: Fentanyl, which is commonly found in "heroin" and adulterated "oxycodone" tablets, was only reported to be detectable by $27.2 \%$ of hospital EDs. As the use of synthetic opioids increases, there is an increased need for improved UDS techniques and enhanced hospital performance. This study is limited by an incomplete hospital response rate and by the potential for error in the respondents' knowledge of their hospitals' opioid test capabilities.

089. Heroin Overdose Trends in a High Volume Urban Emergency Department over a 4-Year Period: 2014-2017

Lydia Baltarowich $^{1}$, Diana Dean ${ }^{2}$, Jessalyn Fontana ${ }^{1}$, Ahsan Akram ${ }^{1}$, Sami Shayya ${ }^{1}$, Joseph Miller ${ }^{1}$
${ }^{1}$ Henry Ford Hospital, Detroit, MI, USA. ${ }^{2}$ Henry Ford Hospital, Detroit, MI, USA

Background: Urban emergency departments (ED) have experienced increasing visits for heroin overdoses since 2014, with noted federal reports that most involve heroin adulterated with fentanyl. Our study objective was to characterize this evolving trend in an urban trauma center ED from 2014 to 2017.

Methods: This is a retrospective observational study of ED patients from 2014 to 2017 with a diagnosis of heroin overdose. Patients in cardiac arrest were excluded. Trained abstractors collected standardized data on clinical information, substance use, and ED disposition. We assessed changes in overdose patterns over time and compared characteristics of admitted and discharged patients. Analysis included descriptive statistics and generalized linear or mixed models where appropriate.

Results: A total of 900 patients were included. The incidence of heroin overdose visits increased twofold between 2015 and 2016 and threefold between 2014 and 2017. During the overall period, 73\% of patients were discharged from ED, $11 \%$ left AMA, and $16 \%$ were admitted. Mean LOS in ED was 6.6 hours. The mean age increased from 47.7 years in 2014 to 50 in $2017(p<0.01)$. Gender, race, and ED disposition did not change significantly. Concomitant drugs on urine screens were opiates $87 \%$, cocaine $46 \%$, benzos $35 \%$, THC $31 \%$, and ethanol $23 \%$. Comparison of admitted and discharged patients showed similar age, gender, and race. Admitted patients were more likely to have concomitant amphetamine use $(p=0.037)$ and less likely THC ( $p=0.006) ; 14 \%$ of admitted patients required ICU care. Respiratory compromise was the most common complication; $66 \%$ of patients with an abnormal chest X-ray had pneumonia or pulmonary edema.

Conclusion: This study shows a significant increase in ED patient visits for heroin overdose from 2014 to 2017 with increasing mean age over time. Most patients were discharged from the ED, and of those admitted, the majority went to non-ICU beds.

090. Ten-Year Trends in Reporting of Methamphetamine and Heroin Exposure as Treated by Medical Toxicologists: 2010-2019

Sharan Campleman ${ }^{1}$, Shao $\mathrm{Li}^{1}$, Jeffrey Brent ${ }^{2}$, Diane Calello ${ }^{3}$, Paul $\mathrm{Wax}^{4}$, On Behalf of the ToxIC Investigators Consortium (ToxIC)

${ }^{1}$ American College of Medical Toxicology, Phoenix, AZ, USA. ${ }^{2}$ University of Colorado School of Medicine, Denver, CO, USA. ${ }^{3}$ Rutgers New Jersey Medical School, Newark, NJ, USA. ${ }^{4}$ University of Texas, Southwestern Medical Center, Dallas, TX, USA

Background: Opioids (OPI) and sympathomimetics are two of the most common agent classes reported to the Toxicology Investigators Consortium, including drugs of abuse such as heroin (HER) and methamphetamine (METH). Previously, a 6-year analysis observed a decline in cases involving any OPI. In addition, the absolute number of cases involving METH appeared to be rising. Therefore, it is important to update and parse out the relative influence of multiple vs. single-agent exposures over a longer 10-year period. Research Question: What changes in toxic events involving HER and METH have occurred over the 10 years of reporting to the ToxIC Registry? Methods: This descriptive analysis included all ToxIC Registry cases January 1, 2010, through October 31, 2019 reporting exposure to at least one toxic agent. Summary descriptive statistics calculated included annual relative frequencies and chi-square for linear trend.

Results: OPI class agents reported in 13.8 to $18.7 \%$ of cases annually. Linear tests based on the percentage of total agents indicated an overall slight upward trend for any OPI $\left(\chi^{2}=18.949, p<0.001\right)$, but a sharper (slope $>3 \times)$ increase for HER $\left(\chi^{2}=418.04, p<0.001\right)$. For example, in 2010 HER was reported in $1.6 \%$ cases, while in 2019 HER was reported in $8.3 \%$ of cases. The annual proportion of cases reporting METH alone $\left(\chi^{2}=161.73, p<0.001\right)$ or in combination with HER $\left(\chi^{2}=99.758\right.$, $p<0.001)$ also increased significantly through October 2019 .

Conclusion: ToxIC Registry cases involving HER and METH demonstrated significant positive increase in their relative proportion of poisonings. As the Registry continues to increase in size and accumulated data years, the ability to identify stable estimates of time trend has improved. 
Although the overall percent of cases reporting any OPI declined, cases reporting HER increased indicating the expanding use of HER, both with and without concurrent METH. Investigators Consortium.

091. Nobody Wants to Be Narcan'd: a Pilot Qualitative Analysis of Drug Users' Perspectives on Bystander Naloxone

Jeffrey T. Lai ${ }^{1}$, Charlotte E. Goldfine ${ }^{1}$, Brittany P. Chapman ${ }^{1}$, Melissa M. Taylor $^{1}$, Rochelle K. Rosen ${ }^{2}$, Stephanie P. Carreiro ${ }^{1}$, Kavita M. Babu ${ }^{1}$

${ }^{1}$ University of Massachusetts Medical School, Worcester, MA, USA.

${ }^{2}$ Brown University School of Public Health, Providence, RI, USA

Background: Efforts to increase naloxone availability figure prominently in public health strategies to decrease opioid overdose deaths. While there is ample evidence supporting the effectiveness of bystander naloxone distribution programs, the effects of increasing naloxone availability on people who use drugs (PWUD) have not been adequately delineated. This pilot study seeks to (1) evaluate whether individuals' drug use patterns and attitudes have changed as a result of proliferation of naloxone distribution programs and (2) explore individuals' knowledge of, access to, experiences with, and perceptions of naloxone.

Hypothesis: We hypothesize PWUD have familiarity with and accept naloxone use, and do not increase their drug use in the presence of naloxone. Methods: We piloted a semistructured interview developed by the National Drug Early Warning Systems workgroup with participants presenting to a large, tertiary care academic emergency department with a history of nonmedical opioid use. Thematic analysis was used to code and analyze transcripts from the semistructured interviews.

Results: Ten participants completed the study. All participants were aware of naloxone by brand name (Narcan $($ ) and were trained in its use, and all but one possessed a naloxone kit. None of the participants reported an increase in their drug use when naloxone was present. Barriers to naloxone administration included fear of legal repercussions, not having it readily available, and a desire to avoid interrupting another user's "high." Seven out of ten participants reported having previously received naloxone at least once. All participants who had received naloxone described an intensely unpleasant physical response consistent with severe opioid withdrawal.

Conclusion: Participants were accepting of, knowledgeable about, and willing to administer naloxone. We discovered that participants did not increase their use of opioids when naloxone was available. Instead, they reported a tendency to use opioids more cautiously due to fears of precipitated withdrawal from receipt of naloxone.

092. Treatment of a Cocaine-Fentanyl/Cocaine-Acetylfentanyl Overdose Event Reaching International Media Recognition

William J Trautman, Rachael C Westover, Michael G Abesamis University of Pittsburgh, Pittsburgh, PA, USA

Background: Recent public health data shows increased morbidity and mortality from heroin, fentanyl, and fentanyl analogues. Single ingestion, multipatient events are rare and offer an opportunity to understand the varied presentation and publicized reactions.

Hypothesis: Multiple patients hospitalized from a single drug exposure lead to increased media and legal scrutiny of common exposures.

Methods: This is a case series of three patients presenting with an unknown overdose. Three additional patients were dead on scene. All were wearing orange wristbands. All patients received prehospital naloxone for respiratory depression.

Results: Laboratory studies demonstrated significant anion gap metabolic acidosis with elevated lactate $(9.4-12.6 \mathrm{mMol} / \mathrm{L})$ in addition to multiorgan dysfunction: creatinine 1.70-2.14 mg/dL, AST 95-763 units/L, ALT 119658 units $/ \mathrm{L}$, and troponin $<0.04-0.38 \mathrm{ng} / \mathrm{mL}$. They were loaded with fomepizole for the metabolic derangements. One patient required intubation for airway protection and was discharged after 3 days. The other patients were admitted for IV fluids and anti-emetics with resolution of laboratory abnormalities within 2 days. Gas chromatography-mass spectrometry of the patients' urine showed cocaine, acetylfentanyl, and fentanyl, with cocaethylene present in two patients. Toxic alcohol testing was negative. The FBI and DEA interviewed staff and patients regarding substance identification, safe scene evaluation, and treatment staff safety. Outside media released national and international reports reflecting opioid exposure concerns and inquired if the wrist bands were gang related. The patients later admitted to cocaine use from a package testing positive for fentanyl analogues. The wristband was a local bar item used to identify patrons who were carded for alcohol consumption. Conclusion: Cocaine adulteration with fentanyl analogues can present with a spectrum of illness including death and multiorgan dysfunction responsive to supportive care. Media and law enforcement responses to this event showed a trend toward sensationalizing exposures and international inquiry despite typical presentation.

\section{Addiction Medicine Training in US Medical Toxicology Fellowships}

Joseph E Carpenter ${ }^{1,2}$, Nichole Lang ${ }^{1}$, Brent W Morgan ${ }^{1,2}$, Timothy J Wiegand ${ }^{3}$

${ }^{1}$ Emory University, Atlanta, GA, USA. ${ }^{2}$ Georgia Poison Center, Atlanta, GA, USA. ${ }^{3}$ University of Rochester Medical Center, Rochester, NY, USA

Background: A growing subset of medical toxicologists (MT) are making addiction medicine (AM) a key part of their clinical practice. Approximately $8 \%$ of currently board-certified MT hold dual certification in AM, most via an AM "practice pathway." The American Board of Emergency Medicine (ABEM) and the American Board of Preventive Medicine (ABPM) recently approved a 30-month fellowship track combining MT and AM. In a 2015 survey of US MT fellowship directors, none reported having an AM-boarded faculty member, three had an X-waivered faculty member, three offered an Xwaiver course as part of the fellowship, and only one had a faculty member that prescribed buprenorphine. We sought to determine current AM-related faculty credentials and educational opportunities in US MT fellowships.

Methods: We sent a 25-question survey to all 29 US MT fellowship directors via the ACMT fellowship listserv in October 2019 and sent two reminder emails over the following month. The survey was hosted on REDCap and used a combination of multiple-choice and free-response questions.

Results: Seventeen of $29(59 \%)$ fellowships responded, representing $60 \%$ of active MT fellows. Six programs reported there is a separate AM fellowship at their institution, and one offers a combined fellowship in MT and AM. Compared to 2015, the number of programs with at least one AM-boarded faculty member increased from three to eight, those with an X-waivered faculty member increased from three to 17 , and those offering an X-waiver course during the fellowship increased from three to 17 . Seven require fellows to complete an $\mathrm{X}$-waiver course. The number of programs with physicians prescribing buprenorphine also increased from one to 14 .

Conclusion: An increasing number of US MT fellowship programs have faculty members with specialized certification in AM and offer AMrelated educational opportunities. Several appear well-positioned to form combined fellowships in MT and AM.

\section{Kratom (Mitragynine speciosa) Induced Acute Liver Failure}

Steven J Fishburn, Michael E Mullins

Washington University, St Louis, MO, USA

Background: Kratom is an herbal product derived from the Mitragynine speciosa plant. The psychoactive properties of kratom are mediated by opioid receptor agonist activity. Toxicity has been well described in the literature. Our recent case demonstrated acute liver failure with liver enzymes well above those previously reported. 
Methods: This is a single patient chart review. A 36-year-old female with a past medical history of depression, bipolar disorder, and high blood pressure presented after for intentional overdose of alprazolam, zolpidem, and kratom. Initial laboratory evaluation showed elevated liver enzymes (AST $1804 \mathrm{IU} / \mathrm{L}$, ALT $6528 \mathrm{IU} / \mathrm{L}$, total bilirubin $12 \mathrm{mg} / \mathrm{dL}$ ), acute kidney injury (creatinine $9.57 \mathrm{mg} / \mathrm{dL}$ ), and coagulopathy (INR 3.3 ). Acetaminophen and salicylate levels were obtained and were negative. Patient was intubated, admitted to the ICU for several days, and received acetylcysteine $150 \mathrm{mg} / \mathrm{kg}$ bolus with a $12.5-\mathrm{mg} / \mathrm{kg}$ /hour infusion. Liver enzymes improved and acute kidney injury recovered without dialysis. The patient was trialed on methadone for opioid withdrawal symptoms on day 4 of admission. She recovered from her acute overdose and was transferred to the Hospital Medicine floor on day 7 after ingestion.

Results: Serum mitragynine levels were obtained from blood sampled during the initial evaluation of the patient. Mitragynine (>500 ng/ml) presence of 7-hydroxymitraginine was confirmed in the sample.

Conclusion: We describe serum liver enzyme levels that were well above previously reported with kratom ingestion toxicity.

\section{More Than Just a Pick Me Up: a Case of Caffeine Toxicity}

Colin Therriault ${ }^{1,2}$, Matthew Eisenstat ${ }^{1,2}$, Michael Yeh $^{1,2}$, Brent Morgan $^{1,2}$

${ }^{1}$ Emory University, Atlanta, GA, USA. ${ }^{2}$ Georgia Poison Center, Atlanta, GA, USA

Background: Caffeine is a trimethylxanthine which exerts its effect primarily through nonselective inhibition of adenosine receptors and endogenous catecholamine release. With new formulations of caffeine, cases of its toxicity are becoming more common.

Methods: This is a single patient case review. A 23-year-old man with history of methamphetamine abuse ingested $4000 \mathrm{mg}$ of caffeine in a suicide attempt. On presentation, his vital signs are as follows: HR 179 bpm, BP 82/ $52 \mathrm{mmHg}$, RR 30/minute, and $\mathrm{SpO}_{2} 98 \%$. He was noted to have pinpoint pupils, diaphoresis, and poor mentation. He was treated with $6 \mathrm{mg}$ lorazepam and given $4 \mathrm{~L}$ normal saline. His electrocardiogram (ECG) showed atrial fibrillation with numerous premature ventricular complexes with a rate of $155 \mathrm{bpm}, \mathrm{QRS}$ of $113 \mathrm{~ms}$, and QTc of $495 \mathrm{~ms}$. Thirty minutes after arrival, he became hypotensive and had an episode of ventricular tachycardia, which was terminated with cardioversion at $100 \mathrm{~J}$. Afterwards, his telemetry monitor continued to show significant ectopy, tachycardia, and hypotension. He was started on $50 \mathrm{mcg} / \mathrm{kg} /$ minute of esmolol and $50 \mathrm{~g}$ activated charcoal.

Results: After starting esmolol, his vital signs and mental status improved. Lab work was notable for a potassium of $2.4 \mathrm{mEq} / \mathrm{L}$ and bicarbonate of $19 \mathrm{mmol} / \mathrm{L}$. His caffeine level was $>90 \mathrm{mg} / \mathrm{L}$ (for reference a daily coffee drinker has caffeine levels of $1-10 \mathrm{mg} / \mathrm{L}$ ). A repeat ECG showed normal sinus rhythm with a QRS of 113 and QTc of 512. His esmolol was discontinued after 27 hours and he was evaluated by a psychiatrist who determined his suicide attempt was due to methamphetamine use and not appropriate for inpatient psychiatric admission. The patient was discharged on hospital day 3 .

Conclusion: This case adds to the current literature of the role of beta blocker therapy in the treatment of methylxanthine toxicity.

096. Tracking Methamphetamine Trends: Comparison of Illinois State Police Methamphetamine Seizures Vs Methamphetamine Cases Reported to the Illinois Poison Center

Elizabeth Black ${ }^{1}$, Marit S Tweet ${ }^{1}$, Michael S Wahl ${ }^{2}$

${ }^{I}$ Toxikon Consortium, Chicago, IL, USA. ${ }^{2}$ Illinois Poison Center, Chicago, IL, USA

Background: Methamphetamine abuse and mortality are increasing in the USA. CDC reports have referred to this increase as the "fourth wave" of drug overdose deaths. Different data sets can be used to track methamphetamine use trends.
Hypothesis: There is a correlation between the amount of methamphetamine seized by state police to methamphetamine exposures reported to the Illinois Poison Center (IPC).

Methods: We compared the number of IPC cases involving methamphetamine to total grams of methamphetamine seized and submitted to state laboratory over a 9-year period (2009-2017).

Results: From 2009 to 2017, there was a $123 \%$ increase in amount of methamphetamine seized $(15,239$ to $34,054 \mathrm{~g})$ and a $508 \%$ increase in the number of cases reported to the RPC ( 25 cases to 152 cases).The number of IPC cases per $1000 \mathrm{~g}$ of methamphetamine seized increased $172 \%$, from 1.64 cases $/ 1000 \mathrm{~g}$ in 2009 to $4.46 \mathrm{cases} / 1000 \mathrm{~g}$ in 2017 ; this increase was steepest in the most recent 5-year period. From 2014 to 2017, there has been a statistically significant correlation between the increase of methamphetamine seized to the increase in reported IPC cases (CC 0.98, $p$ 0.04).

Conclusion: There has been an increase in both police seizures of methamphetamine and in cases reported to the IPC. The increasing amount of methamphetamine seized may be related to increased supply of the drug in the state which is leading to an increased number of methamphetamine exposures reported to the IPC. The rate of symptomatic cases reported to the IPC increased at a much higher rate than the amount of methamphetamine seized by state police. Further analysis and research will be needed to better examine the linkage and utility of these databases in tracking this re-emerging epidemic.

097. Increase in UK Emergency Department Presentations Related to Acute Methamphetamine: Concern for the Future?

James T Harnett ${ }^{1}$, Paul I Dargan ${ }^{1,2}$, Alison M Dines ${ }^{1}$, John RH Archer ${ }^{1,2}$, Shaun L Greene ${ }^{1}$, David M Wood ${ }^{1,2}$

${ }^{I}$ Guy's and St Thomas' NHS Foundation Trust, London, UK. ${ }^{2}$ King's College London, London, UK

Background: In the USA, there have been concerns about the widespread use of methamphetamine. Previous studies of UK-based population/subpopulation level surveys, work-place drug testing, and ED presentations have not shown similar issues related to the use of methamphetamine in the UK. More recently, there has been a resurgence of concerns about increasing use and associated harms related to use of methamphetamine in the UK.

Hypothesis: Methamphetamine-related acute toxicity and ED presentations are increasing in the UK.

Methods: This is a retrospective analysis of prospectively collected data on ED presentations related to acute methamphetamine toxicity to two inner London hospitals, between 2005 and 2018. Data from identified cases were extracted from presentations between 2015 and 2018 including demographics, co-used substances, clinical features, and outcomes.

Results: The number of presentations increased from 4 in 2005 to 294 in 2018. Of the 850 presentations between 2015 and 2018, 94.9\% $(n=807)$ were male and mean (range) age was 35.1 (16-67) years. Methamphetamine presentations were associated with severe cardiovascular toxicity, with 33.1 and $47.6 \%$ having significant systolic hypertension (> $140 \mathrm{mmHg}$ ) or tachycardia (> $100 \mathrm{bpm})$, respectively. Overall, 474 (56.2\% of 843 with available data) methamphetamine presentations were discharged from the ED (medically discharged 398; self-discharged 76). Of the 369 admitted, 57 (15.4\% of those admitted) were admitted to a critical care (level 2 or 3 ) facility. The most commonly co-used substance was GHB/GBL $(n=461,54.2 \%$ of presentations), followed by ethanol $(n=166,19.5 \%)$, mephedrone $(n=95,11.1 \%)$, and cocaine/crack cocaine $(n=68,8.0 \%)$. Co-use of GHB/GBL with methamphetamine attenuated the neurological/neuropsychiatric toxicity seen with lone methamphetamine use: anxiety 26.1 vs $40.6 \%, p=0.002$; hallucinations 12.4 vs $26.1 \%, p<0.001$; and psychosis 12.4 vs $24.6 \%, p=0.001$.

Conclusion: Methamphetamine-related ED presentations with significant acute toxicity are increasing in the UK. An appropriate approach by legislative and other bodies is needed to address this trend and to reduce associated harms.

098. Acute Toxicity Related to Recreational Use of Tramadol: Experience of the European Drug Emergencies Network Plus (Euro-DEN Plus) Project 
Kerry Layne ${ }^{1}$, Paul I Dargan ${ }^{1,2}$, Alison M Dines ${ }^{1}$, Christopher Yates ${ }^{3}$, $\mathrm{Knut} \mathrm{Erik} \mathrm{Hovda}^{4}$, Fridtjof Heyerdahl ${ }^{5}$, Isabelle Giraudon ${ }^{6}$, Euro-DEN Plus Research Group ${ }^{7}$, David M Wood ${ }^{1,2}$

${ }^{1}$ Guy's and St Thomas' NHS Foundation Trust, London, UK. ${ }^{2}$ King's College London, London, UK. ${ }^{3}$ Hospital Universitari Son Espases, Mallorca, Spain. ${ }^{4}$ Oslo University Hospital, Oslo, Norway. ${ }^{5}$ Oslo University Hospital, Oslo, Norway. ${ }^{6}$ European Monitoring Centre for Drugs and Drug Addiction, Lisbon, Portugal. ${ }^{7}$ Euro-DEN Plus Research Group, London, UK

Background: There are increasing reports of tramadol misuse across the world, particularly in the Middle East and North and West Africa. This study describes presentations with acute toxicity related to recreational use of tramadol to EDs across Europe over a 4-year period.

Research Question: To describe the pattern of acute toxicity related to recreational use of tramadol in Europe.

Methods: Data were extracted from the European Drug Emergencies Network (Euro-DEN) Plus database from 1st January 2014 to 31st December 2017 (32 EDs in 21 countries contributed data in this time period) on all presentations with acute toxicity related to tramadol misuse; the following data were extracted: basic demographics, co-used drugs, clinical features at the time of presentation, and treatments/outcomes.

Results: There were 105 presentations in the 4-year period related to recreational use of tramadol; these were most common in Bratislava (Slovakia) ( $n=11,7.5 \%$ of all presentations to this center), Riga (Latvia) $(n=4,4.9 \%)$, and Munich (Germany) $(n=17,2.9 \%)$. There were no cases involving tramadol misuse in 12 centers. In 32 presentations, tramadol was used alone (excluding ethanol). Commonly reported co-used substances were opioids ( $28.6 \%$ of cases), benzodiazepines (21.9\%), and cannabis (16.2\%). On arriv$\mathrm{al}, 14(13.3 \%)$ of presentations were in coma/GCS $\leq 8$ and nine of these had a respiratory rate $<12$ per minute. Seventeen $(16.2 \%)$ patients received naloxone and six $(5.71 \%)$ were intubated. Forty-eight $(45.7 \%)$ patients were medically discharged from the ED; 23 (12.4\%) self-discharged, 16 (15.2\%) were admitted to hospital, $13(12.4 \%)$ were transferred to psychiatry, and five (4.8\%) were admitted to critical care.

Conclusion: There was variability in the number of tramadol-related acute toxicity-related presentations across the Euro-DEN Plus centers with clustering of cases in certain countries. These presentations potentially pose a significant burden on EDs with a large proportion requiring admission to hospital for ongoing care.

099. Massive Gamma Hydroxybutyric Acid Overdose Resulting in Severe Metabolic Acidosis Requiring Continuous Venovenous Haemofiltration

Anselm Wong ${ }^{1,2}$, Mohamed Umar ${ }^{3}$, Sarah Morey ${ }^{4}$, Linda Glowacki ${ }^{4}$, Dimitri Gerostamoulos ${ }^{4}$

${ }^{1}$ Austin Health, Heidelberg, VIC, Australia. ${ }^{2}$ Monash University and University of Melbourne, Melbourne, VIC, Australia. ${ }^{3}$ Eastern Health, Melbourne, VIC, Australia. ${ }^{4}$ Victorian Institute of Forensic Medicine, Melbourne, VIC, Australia

Background: Severe metabolic acidosis from gamma-hydroxybutyric acid (GHB) overdose is rare.

Hypothesis: Dialysis can remove GHB and is a useful treatment in massive overdose.

Methods: A 31-year-old male presented to the hospital emergency department via ambulance half an hour after taking $250 \mathrm{~mL}$ of GHB with suicidal intent. $\mathrm{He}$ showed signs of gross psychomotor agitation requiring physical and pharmacological restraint with intramuscular midazolam and droperidol. An initial blood gas showed $\mathrm{pH} 7.24$, bicarbonate $20 \mathrm{mmol} / \mathrm{L}, \mathrm{pCO}_{2} 46 \mathrm{mmHg}$ and lactate $4.4 \mathrm{mmol} / \mathrm{L}$. He became sedated and, over subsequent hours, had several periods of apnoea and was intubated.

Results: In the intensive care unit (ICU), his blood gases were $\mathrm{pH}$ 6.81, bicarbonate $8 \mathrm{mmol} / \mathrm{L}, \mathrm{pCO}_{2} 51 \mathrm{mmHg}$ and lactate $2.9 \mathrm{mmol} / \mathrm{L}$. Continuous venovenous haemodiafiltration (CVVHDF) was commenced at 12 hours post- ingestion and continued for 9 hours. The blood GHB concentration at 9 hours post-ingestion prior to CVVHDF was $2300 \mathrm{mg} / \mathrm{L}$. The extraction ratio of GHB at 17 hours post-ingestion was $46 \%$ with a plasma clearance of $54.3 \mathrm{~mL} /$ minute on CVVHDF (blood flow rate and ultrafiltration rate $200 \mathrm{~mL} /$ minute). The apparent half-life of GHB whilst on CVVHDF was 3.5 hours compared to 5 hours after it was ceased. His acid-base status improved and he was extubated on day 2 of his ICU admission. He was discharged to the inpatient psychiatric unit under a treatment order on day 3. GHB has a low molecular weight, small volume of distribution and minimal protein binding. This coupled with the ability to achieve clearance during dialysis of GHB may make this a reasonable treatment option in massive overdose and decreasing length of hospital stay and morbidity.

Conclusion: Massive GHB overdose can result in severe metabolic acidosis. Dialysis with CVVHDF can assist with removing GHB and correct the acid-base disturbance.

\section{Trends in Use and Harms of Alkyl Nitrites and an Evaluation of Causes of Adverse Events}

Jared A Brown, Amy Peacock

UNSW, Sydney, NSW, Australia

Background: Alkyl nitrites are currently subject to scrutiny by regulators across the globe due to emerging evidence of vision loss, but data on usage and toxicity is poorly described in a large general population cohort.

Methods: This is a retrospective observational study analysis of Australian National Drug Strategy Household Survey (NDSHS), Ecstasy and Related Drugs Reporting System (EDRS), Poisons Information Centres (PIC), New South Wales Emergency Department Surveillance (NSWEDS) and National Coronial Information System (NCIS) over 2000-2018.

Results: Recent use in the NDSHS has increased by $50 \%$ in the past decade, with 2016 population rates of 3.4 per 1000 . Rates of recent usage reported by stimulant users showed no trend with time (14-29\%). Homosexual/bisexual men and women were more likely to report use, but heterosexuals still represented up to three quarters of those reporting use. PIC cases quadrupled in the past decade, with 2017-2018 doubling. Variability in geographical distribution was seen with eight-fold variation by state (maximum 1.6 per 1000 PIC calls). Approximately two thirds of people reporting use and harms were male and has remained relatively constant over time. NSWEDS cases in women occurred at a younger age than men. The majority of PIC cases related to accidental ingestion of liquid $(50.5 \%)$ followed by inhalation $(18.7 \%)$, intranasal $(12.4 \%)$ and ocular (12.4\%). Jungle Juice was the most common product. Hospitalization was needed for all routes of administration (64\%), with the highest rates for ingestion. Alcohol intoxication was recorded in half of the PIC cases and more commonly used as a party drug vs sex aid. Onset and offset of toxicity was rapid, with $83 \%$ discharged promptly from ED. Three deaths were identified in the NCIS.

Conclusion: Increasing cases of poisoning in Australia is concerning and signals the need for increased vigilance worldwide. Harms from wrong route errors highlight the need for harm minimization strategies.

\section{A Pilot Trial of Topical Capsaicin Cream for Treatment of} Cannabinoid Hyperemesis

Diana J Dean ${ }^{1,2}$, Noor Sabagha ${ }^{2}$, Kaitlin Rose ${ }^{2}$, Alexander Weiss ${ }^{2}$, Timothy Asmar ${ }^{2}$, Jo-Ann Rammal ${ }^{2}$, Margaret Beyer ${ }^{2}$, Rebecca Bussa ${ }^{2}$, Thomas Smoot ${ }^{3}$, Joseph Miller ${ }^{2}$

${ }^{1}$ Michigan Poison Center at Wayne State University, Detroit, MI, USA. ${ }^{2}$ Henry Ford Hospital, Detroit, MI, USA. ${ }^{3}$ Frederick Memorial Hospital, Frederick, MD, USA

Background: Patients with cannabinoid hyperemesis syndrome (CHS) frequently present to the emergency department. Previous case studies suggest dramatic symptomatic improvement with topical capsaicin treatment. 
Research Question: This exploratory study examines the safety and potential efficacy of topical capsaicin in patients with vomiting due to a suspected CHS exacerbation.

Methods: This is a double-blind, randomized placebo-controlled pilot trial. Adults who presented with vomiting suspected to be from CHS were eligible for enrollment. We excluded pregnant females and those with resolution of nausea. Following randomization, topical $0.1 \%$ capsaicin or placebo cream was applied to the anterior abdomen in a uniform manner. Primary outcome was the severity of nausea on a visual analog scale (VAS) of 0-10 assessed at 30 minutes. Secondary outcomes were occurrence of post-treatment vomiting, nausea by VAS at 60 minutes, and hospital admission.

Results: This pilot trial enrolled 30 patients: 17 in the capsaicin arm and 13 in the placebo arm. One patient in the capsaicin arm did not tolerate treatment due to skin irritation. Nausea severity at 30 minutes was 4.1 (95\% CI 2.8-5.4) in the capsaicin arm and 6.1 (95\% CI 4.1-8.1) in the placebo arm. At 60 minutes, nausea severity was 3.2 (95\% CI 1.6-4.8) vs. 6.4 (4.7-8.1). The percent reduction in nausea at 60 minutes from baseline was $46.0 \%$ (95\% CI 25.5 $66.5 \%$ ) in the capsaicin arm and $24.9 \%$ (95\% CI 7.8-41.9\%) in the placebo arm. Hospital admission was necessary for four patients in the capsaicin arm $(23.5 \%)$ vs. five $(38.5 \%)$ in the placebo arm (RR $0.61,95 \%$ CI $0.20-1.84$ ). Conclusion: In this pilot trial of topical capsaicin for CHS, capsaicin was well-tolerated and demonstrated signs of efficacy. Further trials are warranted.

\section{Trends in Marijuana Exposures Reported to the Michigan} Poison Center from 2014 to 2019

Diana J Dean ${ }^{1,2}$, Elizabeth Jacobs ${ }^{1,2}$, Andrew M King ${ }^{1,2}$, Cynthia K Aaron $^{1,2}$

${ }^{1}$ Michigan Poison Center, Detroit, MI, USA. ${ }^{2}$ Wayne State University, Detroit, MI, USA

Background: Michigan legalized recreational marijuana December 6, 2018. Similar to other states where marijuana was legalized or decriminalized, Michigan has experienced an alarming increase in pediatric marijuana exposures.

Methods: This is a retrospective review of cases reported to the Michigan Poison Center (MiPC) from 2014 to present. The state of Michigan has 10 million people and one poison center.

Results: From 2014 to 2018, MiPC recorded 1915 cases of marijuana exposures. Total annual case volume doubled between 2014 and 2015, from 161 to 348 cases, and continues to increase. Between 2014 and 2018 , the number of pediatric cases ( $<18$ years old) nearly tripled (46 to 120 ) and were mainly due to edibles. In fact, $58 \%$ of the 110 edible exposure cases occurred in children. Pediatric edible exposures increased from one (2014) to 46 cases (2018). Preliminary data from 2019 continues to show increasing call volumes for all types of marijuana exposures. THC vaping calls increased in 2019 , but remain $<3 \%$ of total calls. Discussion: Marijuana cases reported to the MiPC the years immediately preceding legalization legislation for recreational use were already uptrending. Most concerning was the striking increase in pediatric marijuana exposures and is a likely result of increased access and availability. This emerging pediatric health threat should be met with novel and aggressive methods to limit exposure and resulting toxicity. Limitations include but are not limited to reporting bias, as cases were voluntarily called to MiPC. Additionally, as time goes on, presumably providers will become more comfortable with management and will not continue to report cases with the same frequency.

Conclusion: Since 2014, marijuana exposures reported to the MiPC have increased, especially among pediatric patients, in the period leading up to and after legalization.

103. Perceptions of Emergency Department Patients with Reported Marijuana Use Since Recreational Legalization in Massachusetts

James D Whitledge ${ }^{1}$, Michael Ganetsky ${ }^{2}$, Ryan C Burke ${ }^{1}$, Katherine L Boyle $^{2}$
${ }^{1}$ Department of Emergency Medicine, Beth Israel Deaconess Medical Center, Boston, MA, USA. ${ }^{2}$ Division of Medical Toxicology, Department of Emergency Medicine, Beth Israel Deaconess Medical Center, Boston, MA, USA

Background: In 2016, Massachusetts legalized recreational marijuana use, and dispensaries opened in 2018. The impact of legalization on emergency department (ED) patients is still unclear.

Research Question: What are the perceived harms and benefits, impact of legalization, and potential discordance between patient- and clinicianperceived reason for ED visit in patients using marijuana?

Methods: This is a prospective convenience sample survey study at a single academic medical center ED. Patients screening positive at triage for marijuana were administered a survey on marijuana use patterns, reasons for use, perceived adverse effects, and legalization impact. Reason for ED visit was not limited to patient-perceived marijuana-related adverse effects. Treating physicians were asked whether ED visits were marijuana-related. Statistics were descriptive with Fisher's exact, chi-square, Wilcoxon-Mann-Whitney, and Kruskal-Wallis tests for comparisons.

Results: Seventy-five patients were enrolled over 3 months. Seventy-five percent of patients did not increase use after legalization. Predominant reported marijuana-related adverse effects were anxiety (53\%), abdominal pain (43\%), and vomiting (32\%). Thirty-five percent experienced abdominal pain improving with hot showers. Most common reported recreational use benefits were anxiolysis, relaxation, and improved mood. More patients used medicinal marijuana for pain control compared to recreational users (70 vs. $11 \%$, $p<0.0001$ ). Only $19 \%$ of patients believed marijuana impairs driving and $16 \%$ believed marijuana is addictive. In eight patients, the ED clinician said presentation was marijuana-related; only three of those patients reported the visit was marijuana-related.

Conclusion: Most patients did not increase marijuana use after legalization. In general, medicinal marijuana was used for perceived pain benefits, while recreational was used for psychosocial reasons. Patients tended to minimize intoxicated driving and addiction risks. Discordance existed between patient and clinician perception of ED visit reason for cannabis-related visits, although this requires further investigation because of small numbers.

104. Characteristics and Attitudes Regarding Cannabis Use Among Physicians: Pilot Survey

Ophir Lavon

Carmel Medical Center, Haifa, Israel. Technion-Israel Institute of Technology, Haifa, Israel

Background: Cannabis use is prevalent for both recreational and medical purposes. Data regarding this use among physicians is limited.

Research Question: What are the characteristics of use and personal attitudes regarding cannabis among physicians?

Methods: This is a single medical center, questionnaire-based pilot survey among physicians at all levels of seniority and training. Participants filled the questionnaire anonymously. Collected information included demographics, personal habits, and opinions regarding statements on cannabis. The data were subjected to descriptive statistical analysis.

Results: The survey was completed for 193 physicians. Most of them (70\%) were either interns or residents. Only $12 \%$ were active cigarette smokers; $23 \%$ reported alcohol drinking at least once a week. The clear majority (96\%) denied any other drug abuse except from cannabis. Eleven $(5.7 \%)$ participants reported that they are current active users of cannabis; another $35 \%$ did it rarely or in the far past. The indication for use of cannabis was recreational in $96 \%$. Most of the participants supported the statement that cannabis is a legitimate effective pharmacological compound; less (about third) agreed that recreational cannabis may decrease alcohol and drug abuse. About half (55\%) do not have any problem with the use of cannabis by physicians, if it is not during work. Even more (72\%) agree (completely or partially) that the formal attitude 
toward cannabis use should be more permissive. More than half (60\%) support legalization of recreational use of cannabis. On the other hand, $63 \%$ think cannabis can cause psychogenic dependence. About one third (36\%) agree that cannabis use may be involved with long-term adverse effects or physical dependence.

Conclusion: In this focused survey, only the minority of physicians reported being active cannabis users. Yet, the majority had liberal opinions regarding this use, including by medical personnel, and were less educated on the potential harm of cannabis.

\section{Acute Cannabis Exposures in Adults $>60$ Years Old}

Robert G Hendrickson ${ }^{1,2}$, Matthew J Noble ${ }^{1,3}$

${ }^{I}$ Oregon Health and Science University, Portland, OR, USA. ${ }^{2}$ Oregon Poison Center, Portland, OR, USA. ${ }^{3}$ Oregon Poison Center, Portland, OR, USA

Background: Cannabis exposure in older adults is poorly studied. Research Question: What is the epidemiology, product type, and clinical effects of cannabis exposure in adults $>60$ years?

Methods: This is a descriptive study of acute cannabis exposures in patients > 60 years. Patients were identified by PC staff and a data collection instrument was added to the PC chart and filled with source of the product, type of product, dose, clinical effects, and disposition. PC charts were searched for cannabis in the substance data field and data extracted.

Results: Twenty-five patients were identified over a 16-month study period with an age range of $60-96 ; 60 \%(15 / 25)$ aged $60-69,24 \%$ $(6 / 25)$ aged 70-79, 8.0\% (2/25) aged 80-89, and 8.0\% (2/25) over 90. Route was known in $(92 \%, 23 / 25)$ patients. Exposures were mostly ingestions $[83 \%$ (19/23) vs. $17 \%$ (4/23) inhalation]. Ingestions were mostly edibles $[74 \%(14 / 19)$ vs. $26 \%(5 / 19)$ concentrates]. Inhalation was $75 \%$ (3/4) botanical material and 25\% (1/4) concentrate. Edibles types were $50 \%$ (7/14) brownies, $14 \%$ (2/14) cookies, and one each of butter, chocolate, caramel, tea, or unknown. Source was described in eight edibles and was mostly commercial $(63 \%, 5 / 8)$ vs. homemade $(38 \%, 3 / 8)$. Neurotoxicity was seen in all patients: CNS sedation $(56 \%, 14 / 25)$, altered mental status (44\%, 11/25), CNS excitation (32\%, 8/25), anxiety $(20 \%, 5 / 25)$, and paranoia $(8 \%, 2 / 25)$ (some had $>1$ sign). Severe toxicity: An 87-year old ingested a THC brownie and developed new atrial fibrillation with RVR. A 60year old ingested $\sim 500 \mathrm{mg}$ "Rick-Simpson-Oil" and developed obtundation. A 70-year old developed STEMI and ventricular tachycardia while vaping $65 \%$ THC concentrate and died. Disposition: Twenty-eight percent (7/25) remained home, 52\% (13/25) were observed in an ED and discharged, 16\% (4/25) admitted to ward/OBS, and one died.

Conclusion: Older adults may develop acute cannabis toxicity and most are exposed via ingestion of edibles. Older adults have the potential for severe CNS sedation, myocardial ischemia, and atrial and ventricular dysrhythmias.

106. Dangerously Chilled Out: Pediatric Cannabis Ingestion Presenting with Hypothermia

Sean D McCann ${ }^{1}$, Steven E Aks ${ }^{1,2}$

${ }^{1}$ Toxikon Consortium, Chicago, IL, USA. ${ }^{2}$ Cook County Health, Chicago, IL, USA

Background: Cannabis has been legalized for recreational or medical use in 33 states and Washington, DC. In these states, pediatric cannabis exposure rates have increased. Pediatric patients with cannabis toxicity may present with unexpected signs and symptoms compared to adult patients.

Hypothesis: Cannabis intoxication in pediatric patients may be associated with marked vital sign abnormalities, including hypothermia, and documentation of quantitative laboratory concentrations would be of value to correlate with clinical effects. Methods: This is a single patient case report. A 6-year-old girl with no medical problems was left with a babysitter overnight. The next morning the patient was found by her mother in her bed, on her hands and knees, crying, with tense, cool extremities. In the emergency department, she was obtunded. Vital signs were respiratory rate 14 breaths per minute, heart rate 84 beats per minute, blood pressure $102 / 67 \mathrm{mmHg}$, and, most significantly, rectal temperature was $31.3^{\circ} \mathrm{C}$. The patient was intubated for impending airway compromise. A urine drug screen was positive only for tetrahydrocannabinol (THC), and the patient's mother reported cannabis-containing cookies in the house. The patient had no access to any other known medications. Blood was sent for a quantitative cannabinoid panel (NMS Labs, Horsham, PA) and the patient was transferred for PICU admission. Results: Blood cannabinoid panel showed $75 \mathrm{ng} / \mathrm{mL}$ delta-9 THC, $38 \mathrm{ng}$ / $\mathrm{mL}$ 11-hydroxy delta-9 THC, and $290 \mathrm{ng} / \mathrm{mL}$ delta- 9 carboxy THC. Hypothermia resolved over the next 4 hours with warming blankets. The patient's mental status improved, and she was extubated later that day and was noted to have no residual neurologic or behavioral abnormalities. She was transferred out of the PICU the following day with ongoing evaluation by children and family services.

Conclusion: Cannabis toxicity presented with hypothermia in a pediatric patient with markedly elevated blood cannabinoid concentrations.

107. What Do You Know About Mary Jane? A Systematic Review of the Current Data on the THC:CBD Ratio

Victoria Zeyl ${ }^{1}$, Kelsey Sawyer ${ }^{2}$, Rachel S Wightman ${ }^{1,3}$

${ }^{1}$ Alpert Medical School of Brown University, Providence, RI, USA.

${ }^{2}$ Brown University, Providence, RI, USA. ${ }^{3}$ Brown Emergency Medicine, Providence, RI, USA

Background: Ratios of delta-9-tetrahydrocannabinol (THC) and cannabidiol (CBD) impact metabolism and therapeutic effects of cannabis. Currently, no states with legalized medical or recreational cannabis consider ratios THC:CBD in regulations. A better understanding of the interactions between THC:CBD is needed before it is used in a medical setting.

Research Question: What ratios of THC:CBD are selected in clinical cannabis trials and what is the rationale?

Methods: This is a systematic literature review of Central, CINAHL, Embase, PsycInfo, and PubMed of the last 10 years of English language medical cannabis publications highlighting THC:CBD ratios. Included were clinical studies of products containing and listing both THC and CBD ratios, percentages, or weighted amounts. Studies only examining THC or CBD in isolation or in which the ratio was not stated or incalculable were excluded. Case reports and series, abstracts, reviews, and meta-analysis were excluded. Nonhuman, nontherapeutic, or studies examining approved cannabis pharmaceuticals were excluded.

Results: Of 479 unique references 11 met inclusion criteria. Diseases assessed: MS, fibromyalgia, ASD, refractory epilepsy, brain activity, Dravet syndrome, complex motor disorder with predominant dystonia, spasticity, schizophrenia/schizotypal/psychosis, and addiction/dependence. THC:CBD ratios listed and/or calculated: 1:0, 22:1, 2:1, 1:1, 1:2, 1:6, 1:9, 1:20, 1:33, $1: 50$, and $0: 1$. Rationale for ratios selected was often not listed, or simply trivialized as the ratios available to patients in the area, or ratios that were pharmaceutically available throughout the study country. One study compared ratios of high and low THC:CBD, but did not specify the ratios.

Conclusion: The medical community has not drawn substantive conclusions nor thoroughly explored THC:CBD ratios for "best practice" treatment of different disease processes and their sequelae. While there is evidence that cannabis provides medical benefits, research is lacking on standardization of medical cannabis use in Western medical practices.

108. First Double Lung Transplant Secondary to Suspected Ecigarette Vaping-Associated Lung Injury

Elizabeth T Jacobs ${ }^{1}$, Diana J Dean ${ }^{1,2}$, Cynthia K Aaron ${ }^{1}$, Bradley D Tilford $^{3}$, Jordan S Schneider ${ }^{3}$, Jeff A Clark ${ }^{4}$, Andrew M King ${ }^{1}$

${ }^{1}$ Michigan Poison Center, Detroit, MI, USA. ${ }^{2}$ Henry Ford Hospital, Detroit, MI, USA. ${ }^{3}$ Children's Hospital of Michigan, Detroit, MI, USA. ${ }^{4}$ St. John Hospital, Detroit, MI, USA

Background: As of November 13, 2019, the CDC has confirmed 2172 cases of E-cigarette vaping-associated lung injury (EVALI): $70 \%$ in males, 
$86 \%$ involving THC, and 64\% involving nicotine. Although there have been 42 deaths, this is the first reported lung transplant in an EVALI patient.

Methods: This is a single case review.

Results: A 16-year-old white male presented to the ED for dyspnea and cough with history of vaping. He was admitted to "hospital \#1" and treated with antibiotics for suspected pneumonia. Additional history revealed use of self-created THC products containing vitamin E. On hospital day 4, he was transferred to the pediatric ICU for worsening hypoxemia and placed on steroids for suspected EVALI. On hospital day 6, he was intubated due to persistent hypoxia and worsening respiratory status. An extensive infectious and inflammatory evaluation was negative. Lung cytology from BAL did not reveal lipid-laden macrophages or other pathology. Due to worsening respiratory status, he was transferred to "hospital \#2" for veno-venous ECMO on hospital day 11. The remainder of organ function was normal. Despite multiple attempts to wean from ECMO, lung function showed no signs of improvement, and he was referred to "hospital \#3" for lung transplant. After 27 days on ECMO (hospital day 39,) he received a double lung transplant for irreversible lung damage and accumulating barotrauma. The patient is now off EMCO, has a tracheostomy, and is neurologically intact. He is able to speak and is undergoing intensive rehabilitation.

Conclusion: Although most cases of EVALI survive and most often show improvement temporally related to steroid administration, EVALI can be relentless and lead to severe lung injury and death. This patient suffered irreparable lung damage despite early presentation, steroids, and early ECMO. Lung transplant can be considered a viable option for severe and refractory cases.

109. Establishing a Rapid Surveillance Response for E-cigarette, or Vaping, Product Use Associated Lung Injury (EVALI) Using the ToxIC Registry

Kim N Aldy ${ }^{1}$, Dazhe $\mathrm{J} \mathrm{Cao}^{1}$, Paul Wax ${ }^{1,2}$, Jeffrey Brent ${ }^{3}$, Diane Calello ${ }^{4}$, Sharan Campleman ${ }^{2}$, On Behalf of the ToxIC Investigators Consortium (ToxIC)

${ }^{1}$ University of Texas Southwestern Medical Center, Dallas, TX, USA.

${ }^{2}$ American College of Medical Toxicology, Phoenix, AZ, USA.

${ }^{3}$ University of Colorado School of Medicine, Denver, CO, USA.

${ }^{4}$ Rutgers New Jersey Medical School, Newark, NJ, USA

Background: Since July 2019, vaping has been linked to thousands of patients with respiratory failure. Data from these cases will broaden our knowledge of this epidemic and could uncover a link to the etiology or provide improved criteria for diagnosis and treatment. With input from the Federal Drug Administration and Centers for Disease Control (CDC), the Toxicology Investigators Consortium (ToxIC) committee created an EVALI form.

Research Question: Can an established registry quickly respond to an emerging public health issue?

Methods: The EVALI form was incorporated into REDCap on October 10, 2019. Members submitted suspected vaping cases they treated since July 2019 onward. We reviewed cases entered up to November 10, 2019, with focus on demographics, vaping exposure circumstances, diagnostics, management, and outcomes.

Results: Eight ToxIC sites entered data on 40 patients from 11 hospitals. All patients were treated by participating medical toxicologists. Ages ranged from 13 to 55 years old (mean 23 years), with $65 \%$ males. Of the 37 with complete records, 18 reported only THC vaping, 2 reported only nicotine vaping, 14 reported vaping both, and 3 had unknown vaping history. No prior medical history of heart or lung disease was reported in $80 \%$ of cases. Chest X-ray was normal in 8 cases, but of the 35 patients with chest computed tomography (CT), all were abnormal with ground glass opacities in 31 and pneumothoraces in 2 . Bronchoalveolar lavage with oil staining found lipidladen macrophages in 6 cases and none in 3 cases. Intubation was required in 11 cases, and extracorporeal membrane oxygenation in 2 . One patient died. Conclusion: We established a surveillance of the EVALI epidemic within a month of the CDC's health advisory release. ToxIC investigators are uniquely positioned to collect clinical bedside data allowing for improved understanding of critical toxicological illnesses.
This research was performed in collaboration with the ACMT Toxicology ToxIC Investigators Consortium.

110. Effects of Patient and Use Characteristics on Symptoms and Outcome in E-Cigarette, or Vaping, Product Use Associated Lung Injury (EVALI)

Kim N Aldy ${ }^{1}$, Dazhe $\mathrm{Cao}^{1}$, Mary Billington ${ }^{1}$, Joshua McFalls ${ }^{1}$, Cherie Obilom ${ }^{1}$, Paul Wax ${ }^{1,2}$, On Behalf of the ToxIC Investigators Consortium (ToxIC)

${ }^{1}$ University of Texas Southwestern Medical Center, Dallas, TX, USA.

${ }^{2}$ American College of Medical Toxicology, Phoenix, AZ, USA

Background: EVALI is an illness associated with vaping delta-9tetrahydrocannabinol (THC) oil and/or nicotine products. Patterns and type of products used vary between patients and have unclear consequences on clinical outcome.

Research Question: How do the patient demographics, pattern of vape use, and type of substance used affect presenting symptoms and clinical outcomes? Methods: We reviewed the Toxicology Investigators Consortium (ToxIC) Registry for EVALI cases seen between August 5, 2019, and October 31, 2019 at our academic institutions. We assessed demographics, vaping product use, and presenting symptoms. Heavy vaping was defined as once or more per day. Severe illness was defined as ICU admission or BiPAP/intubation. Odds ratio (OR) and $95 \%$ confidence interval (CI) were determined by Fisher's exact test.

Results: Twenty-four patients met the Centers for Disease Controlconfirmed case definition for EVALI. Adolescents and adults did not have differences in ICU admission; however, no patient over 18 required BiPAP/intubation $(p=\mathrm{NS})$. All patients reported vaping THC, and 10 also vaped nicotine. THC plus nicotine as compared to THC alone did not lead to increased ICU admission (OR 0.13, CI 0.002-1.64) or BiPAP/ intubation (OR 0.27, CI 0.005-3.95). Heavy vs light vaping did not make a difference in severity of illness as defined by ICU admission (OR 0.7, CI 0.06-10.5) or BiPAP/intubation (OR 0.44, CI 0.03-7.49). Flavored THC was not associated with BiPAP/intubation when compared to use of unflavored $(p=\mathrm{NS}$ ). Vomiting ensued in $81 \%$ of heavy THC users, but only $63 \%$ of light vaping patients reported vomiting $(p=$ NS). Twentyone patients received steroids. Flavoring and heavy vaping were not significantly associated with the need for steroids.

Conclusion: In our EVALI cohort, demographics, pattern of vape use, and type of substance used did not significantly affect presenting symptoms and clinical outcomes.

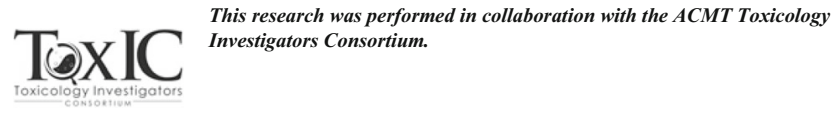

111. Effect of Diagnostic Findings on Treatment and Patient Outcome in E-cigarette, or Vaping, Product Use Associated Lung Injury (EVALI)

Kim Aldy ${ }^{1}$, Dazhe Cao ${ }^{1}$, Mary Billington ${ }^{1}$, Joshua McFalls ${ }^{1}$, Cherie Obilom $^{1}$, Paul Wax ${ }^{1,2}$, On Behalf of the ToxIC Investigators Consortium (ToxIC)

${ }^{1}$ University of Texas Southwestern Medical Center, Dallas, TX, USA.

${ }^{2}$ American College of Medical Toxicology, Phoenix, AZ, USA

Background: E-cigarette, or vaping, associated lung injury (EVALI) is an emerging disease linked to THC oil and/or nicotine product vaping. 
The clinical course, severity, and range of interventions in affected patients around the country have varied widely.

Research Question: How do diagnostic findings affect steroid treatment and clinical outcome in EVALI patients?

Methods: We reviewed the Toxicology Investigators Consortium (ToxIC) registry for EVALI cases seen between August 5, 2019, and October 31, 2019 at our three academic institutions. Severe illness was defined as ICU admission or BiPAP/intubation. Fisher's exact test was utilized to assess diagnostic findings in relation to severity of illness.

Results: Twenty-four patients met the Centers for Disease Controlconfirmed case definition for EVALI. Aspartate aminotransferase (AST) and/or alanine aminotransferase (ALT) was elevated in six patients. AST and/or ALT elevation were not associated with ICU admission ( $p=\mathrm{NS})$. Chest X-ray was performed in all 24 patients. Six patients had a normal initial chest X-ray, with five receiving subsequent chest computed tomography (CT) revealing diffuse ground glass opacities. Normal vs abnormal initial chest Xray was not associated with less ICU admission $(p=\mathrm{NS})$. Bronchoalveolar lavage (BAL) was performed in 10 patients. Eight patient samples were sent for oil red $\mathrm{O}$ staining, and five had positive lipid laden macrophages. None of those with lipoid pneumonia required ICU admission or BiPAP/intubation $(p=\mathrm{NS})$. Five patients received oral steroids and 16 required intravenous steroids. Initial normal chest X-ray did not have a significant effect on the perceived need for oral vs IV steroids $(p=\mathrm{NS})$.

Conclusion: In our population of patients with confirmed EVALI, diagnostic findings did not affect type of steroid treatment started or clinical outcome.

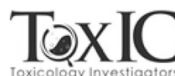

This research was performed in collaboration with the ACMT Toxicology Investigators Consortium.

112. Severe E-Cigarette, or Vaping, Product Use Associated Lung Injury (EVALI) Requiring Veno-Venous Extracorporeal Membrane Oxygenation (VV-ECMO)

Kim N Aldy ${ }^{1}$, Dazhe J Cao ${ }^{1}$, Stephanie Hsu ${ }^{1}$, Molly McGetrick ${ }^{1}$, David Willcutts $^{1}$, Guido Verbeck ${ }^{2}$, Imesha De Silva ${ }^{2}$, Anelle Menendez ${ }^{3}$

${ }^{1}$ University of Texas Southwestern Medical Center, Dallas, TX, USA.

${ }^{2}$ University of North Texas, Denton, TX, USA. ${ }^{3}$ North Texas Poison Control, Dallas, TX, USA

Background: In July 2019, an investigation of cases of acute pulmonary disease of unknown etiology led to a possible association with vaping. EVALI has affected over 2000 otherwise healthy adolescents and adults residing over 49 states and is associated with over 42 deaths to date. We report a severe case of EVALI that was treated aggressively with steroids and required a prolonged course of VV-ECMO.

Methods: A 16-year-old girl presenting with gastrointestinal and respiratory symptoms was admitted to our pediatric intensive care unit (PICU) with progressive respiratory failure and bilateral pulmonary ground-glass opacities on chest computed tomography. After extensive infectious workup was unrevealing, she reported a history of vaping e-cigarettes containing either nicotine or THC oil prior to symptom onset. The PICU team in consultation with pulmonology and medical toxicology started high-dose intravenous (IV) methylprednisolone $1 \mathrm{mg} / \mathrm{kg}$ twice daily for presumed EVALI. Despite initial improvements, she continued to require positive pressure ventilation, developed pneumomediastinum and progressed to tension pneumothoraces with a persistent air leak. Unable to maintain her oxygenation, she was placed on VV-ECMO for a prolonged course. Ultimately, she received a tracheostomy and was discharged with continued vent dependence.

Discussion: The clinical course, severity, and range of interventions in affected patients around the country have varied widely. Respiratory symptoms have been the most severe, but the constellation of symptoms in EVALI include constitutional and gastrointestinal symptoms. In many cases, steroid use led to rapid clinical improvements. However, other cases with severe illness, like our patient, necessitated high-dose IV steroids, intubation, and VV-ECMO. The underlying etiology is likely vitamin $\mathrm{E}$ acetate, but the pathophysiology remains unclear.

Conclusion: Clinicians need to be aware of EVALI and ask about vaping in patients presenting with respiratory and gastrointestinal symptoms. Management strategies are anecdotal and necessitate a multidisciplinary approach.

113. E-cigarette and Vaping-Associated Lung Injury (EVALI) in Pennsylvania and Delaware as Reported to Pennsylvania Poison Centers-2019

Justin Sing ${ }^{1}$, Pok H Tang ${ }^{1}$, Quan Nham ${ }^{2}$, Michael Lynch ${ }^{3}$, Jeanette Trella $^{4}$, Kevin C. Osterhoudt ${ }^{4}$

${ }^{1}$ University Sciences of Philadelphia, Philadelphia, PA, USA. ${ }^{2}$ Wilkes University Nesbitt School of Pharmacy, Wilkes-Barre, PA, USA. ${ }^{3}$ Pittsburgh Poison Control Center, Pittsburgh, PA, USA. ${ }^{4}$ Poison Control Center at The Children's Hospital of Philadelphia, Philadelphia, PA, USA

Background: In 2019, the U.S. Centers for Disease Control recognized an outbreak of lung injury associated with the use of e-cigarette products. The epidemiology of this syndrome is still being characterized.

Research Question: What is the epidemiology of EVALI in PA and DE as reported to poison control centers?

Methods: This is a prospective, descriptive cohort. All probable or confirmed cases of E-cigarette-associated pulmonary illness (NPDS generic code 0077900), meeting case definitions as set forth by the CDC, reported from Delaware and Pennsylvania to their designated poison control centers (PCCs) through October 31, 2019, are summarized with regard to their demographic characteristics, advanced cardiorespiratory support interventions, and outcomes. Results: PA poison centers became aware of their first EVALI case on July 19, 2019, and had a total of 78 cases reported through October 31, 2019. Seventy-three cases were reported from PA and $5(6.4 \%)$ came from DE. The age range of patients was 15 to 57 years (median 21), and $23 \%$ were under 18 years; $69.2 \%$ were male. Fever was reported in $21(29 \%)$ patients, and elevated WBC was noted in $27(37 \%)$. The majority of patients were hospitalized $(91 \%)$ in regular inpatient care unit $74.4 \%$, and $17.9 \%$ in an intensive care unit. Among hospitalized patients, $14.1 \%$ were admitted for more than 1 week. No patients died; $17.9 \%$ were endotracheally intubated, and 5.1\% got ECMO support. Urine toxicology testing was noted as positive for THC in 21 (26.9\%) cases, negative in $5.1 \%$, and was not tested or was unknown in the remainder.

Conclusion: This data set may underestimate the regional incidence of EVALI as many cases may go unrecognized due to lack of standard diagnostic test and the voluntary nature of reporting to poison centers. However, this data shows that the PCCs offer a unique reporting, surveillance, monitoring, and data collection tools during public health crises.

\section{Beads from Snow - a Case of Cerebral Vasospasm from Cocaine Use}

Jaskirat Gill, Laura Tortora

Aventura Hospital and Medical Center, Aventura, FL, USA

Background: Cocaine is a widely abused drug that has remained a major public health problem, with a recent surge in its usage. It binds and blocks monoamine reuptake transporters creating prolonged sympathetic surges. Additionally, by increasing endothelin-1 and nitric oxide concentrations, cocaine can produce vasoconstriction and vasospasm. Here we present a case of cocaine-induced cerebral vasospasm seen on CT angiography.

Methods: This is a single patient chart review. A 30-year-old female with history of weekly cocaine use presented with sudden onset of bitemporal headache that was maximum at onset after she used an unusually large amount of cocaine the night before presentation. Significantly, she had a history of a 
spontaneous aneurysm rupture when she was 8 that required a craniotomy and partial left temporal lobectomy. She had presented within 6 hours of ingestion and CTAs were negative for subarachnoid hemorrhage, but showed beading of the cerebral vessels consistent with cerebral vasospasm. This is a neurologic emergency as patients can have stroke-like presentations and infarct cerebral vascular territories. At the time, her neurologic exam did not reveal any deficits, but she was admitted to the intensive care unit for frequent neurological checks for the immediate 48-72-hour period, and daily transcranial dopplers. She did not require calcium channel blockers, her neurological exams remain normal, and so far, she has had no adverse neurological outcomes.

Conclusion: Cerebral vasospasm is a critical risk of cocaine use and can be associated with significant neurologic sequelae. $\mathrm{CT}$ angiogram may be used to identify these patients early on in their course. Early detection, close monitoring, and early consultation with neuro-interventionalists may be paramount in the treatment of these patients.

\section{Acute Delirium Associated with Transdermal Rotigotine}

Michael Yeh ${ }^{1,2}$, Jared Cavanaugh ${ }^{2,3}$, Matthew D Eisenstat ${ }^{1,2}$, Colin A Therriault ${ }^{1,2}$, Brett de Bruyn ${ }^{2}$, Jonathan de Olano ${ }^{1,2}$

${ }^{1}$ Emory University, Atlanta, GA, USA. ${ }^{2}$ Georgia Poison Center, Atlanta, GA, USA. ${ }^{3}$ Grady Memorial Hospital, Atlanta, GA, USA

Background: Rotigotine is a dopamine receptor agonist used to treat restless legs syndrome (RLS) and Parkinson's disease. There are no known reports of acute transdermal overdose, although symptoms of excessive dopaminergic stimulation may be expected.

Methods: A 62-year-old woman presented with confusion, hallucinations, and multiple falls over the past day. Medical history includes restless legs syndrome, depression, migraines, diabetes mellitus, and thyroid cancer. She was taking ropinirole but ran out 1 week before admission. Initial vitals were BP 156/75, pulse 117 , temperature $37.3^{\circ} \mathrm{C}$, respiratory rate 20 , and glucose $147 \mathrm{mg} / \mathrm{dL}$. She was somnolent, diaphoretic, and hallucinating with akathisia. Labs showed normal CMP, TSH, urinalysis, troponin, PT/INR, ammonia, and lactate. Urine drug screen, ethanol, acetaminophen, and salicylates were negative. Chest X-ray, EKG, and head CT were unremarkable.

Results: The patient initially received IV lorazepam, ketamine, and oral ropinirole $1 \mathrm{mg}$. When she was prepped for lumbar puncture, providers removed two rotigotine $3 \mathrm{mg}$ patches from her skin. Her mental status returned to baseline the following day. She had been prescribed rotigotine $3 \mathrm{mg} / 24$-hour patches for RLS but switched to oral ropinirole due to skin irritation. After running out of ropinirole, she applied two rotigotine $3 \mathrm{mg}$ patches the night before presentation. Conclusion: This case highlights the importance of a complete physical exam in evaluating patients with altered mental status. The patient applied two $3 \mathrm{mg} / 24$-hour patches after having discontinued ropinirole, which likely caused an abrupt increase in dopaminergic stimulation. The initial recommended dose for RLS is $1 \mathrm{mg} / 24$ hours, with maximum of $3 \mathrm{mg}$ / 24 hours. Up to $8 \mathrm{mg} / 24$ hours may be used for Parkinson's disease, but the dose is typically titrated slowly. Transdermal rotigotine, and other dopamine agonists, should be included in the broad differential for patients with acute delirium and symptoms of dopamine excess.

116. Developing and Validating an Accelerated Extracorporeal Membrane Oxygenation (ECMO) Course for Military Critical Care Clinicians Using Yorkshire Swine (Sus scrofa)

Joseph K Maddry ${ }^{1,2}$, Joni A Paciocco ${ }^{1}$, Allyson A Arana ${ }^{1}$, Crystal A Perez ${ }^{1}$, Lauren K Reeves ${ }^{1}$, Kimberly L Medellin ${ }^{1}$, Maria G Castaneda ${ }^{3}$, Patrick C Ng${ }^{1,2}$, Vikhyat Bebarta ${ }^{4}$

${ }^{1}$ United States Air Force En route Care Research Center/59th MDW/ST, San Antonio, TX, USA. ${ }^{2}$ Department of Emergency Medicine, San Antonio Military Medical Center, San Antonio, TX, USA. ${ }^{3}$ Clinical Resuscitation, Emergency Science and Toxicology, Lackland AFB, TX, USA. ${ }^{4}$ Department of Emergency Medicine, University of Colorado School of Medicine, Aurora, CO, USA
Background: Extracorporeal membrane oxygenation (ECMO) is a modification of cardiopulmonary bypass that allows prolonged support of patients with severe respiratory or cardiac failure. ECMO indications are rapidly evolving and there is growing interest in its use for cardiac arrest and cardiogenic shock. Case reports have documented the use of ECMO for calcium channel antagonist, beta-receptor antagonist, and other potentially fatal overdoses. However, most emergency departments lack ECMO equipment or training staff. Training of emergency medicine clinicians could enable the use of ECMO in cardiotoxic overdoses refractory to standard therapies. We aimed to develop and validate an accelerated ECMO course that can be taught to emergency and critical care physicians and nurses.

Methods: We developed a training model using Yorkshire swine (Sus scrofa), a procedure validation checklist, a confidence assessment, and a knowledge assessment. Participants completed an 8-hour ECMO course and were assigned into teams of two (physician + nurse). An ECMO specialist trained them on priming and preparation of the ECMO circuit and oversaw vascular access and ECMO initiation. We used the validation checklist to evaluate performance. Participants completed confidence and knowledge assessments before and after the course.

Results: Seventeen teams (34 clinicians) completed the ECMO course. None had completed an ECMO certification course prior to participation. The validation checklist had $92 \%$ interrater agreement; the confidence and knowledge assessments showed good reliability (Cronbach's alpha = $\left.0.90 ; r_{\mathrm{pb}} \geq 0.20\right)$. All teams primed and prepared the ECMO circuit (mean $31 \pm 6$ minutes). Fifteen teams (88\%) completed the cannulation procedure (mean time $59 \pm 25$ minutes). Two teams could not initiate ECMO due to a fatal arterial laceration. Participants' knowledge and confidence scores improved after completing the course $(p<0.0001)$.

Conclusion: We developed an accelerated 8-hour ECMO course. Clinicians' confidence and knowledge assessments improved after the course and $88 \%$ of teams were able to successfully initiate ECMO.

\section{Day 3: Lightening Orals, Abstracts 117-123}

\section{Utility of Four Smartphone Applications in the Identification of Poisonous Plants}

Lauren Longo ${ }^{1}$, Jules McQueen ${ }^{1}$, Matthew Fox ${ }^{1}$, Than Tam Nguyen ${ }^{1}$, Timothy Block ${ }^{2}$, Kevin C Osterhoudt ${ }^{1}$

${ }^{I}$ The Poison Control Center at Children's Hospital of Philadelphia, Philadelphia, PA, USA. ${ }^{2}$ Morris Arboretum of the University of Pennsylvania, Philadelphia, PA, USA

Background: Foragers, children, and pets may eat plants and risk poisoning. Many people lack plant identification expertise. Smartphones are commonly used to find health information, and several applications (apps) exist that claim to identify plants using phone camera capabilities. Research Question: Are plant identification apps useful to identify poisonous plants?

Methods: Free versions of three popular plant identification apps (iNaturalist, Picture This: Plant Identifier, Plant Snap), plus Google's® "Lens" feature, were used to identify a convenience sample of poisonous and nonpoisonous plants in June in a mid-Atlantic, university-curated arboretum. The smartphone identifications were evaluated by the arboretum's academic Director of Botany. An accuracy statistic was calculated. Results: Fourteen plants (12 poisonous and 2 nonpoisonous) were evaluated with the four apps. The accuracy of "first choice" identification ranged from 8/14 (57\%) to 10/14 (71\%-Plant Snap). Accuracy of identification improved to $11 / 14(79 \%)$ to $13 / 14$ (93\%-iNaturalist) if the correct identification was listed either as a first choice or an alternative; 6/14 plants were precisely identified by all four apps. Poison hemlock was not absolutely identified by any app and was only listed as an alternative on two; water hemlock was not studied. The iNaturalist app performed best with $93 \%$ accuracy [ $95 \%$ CI 66-99\%]. The combined use of 
iNaturalist PLUS either Plant Snap or Google® Lens would have suggested toxicity in all poisonous plants studied.

Conclusion: Smartphone apps correctly identified the majority of poisonous plants investigated. With good positive predictive value, a plant identified as toxic should be avoided. Caution is warranted if a plant is identified as a nontoxic variety; poison hemlock was misidentified by half the apps. Limitations include the use of arboretum specimens and careful photography, seasonal/geographic constraints, and small sample size. Smartphone apps may be a useful adjunct to identify poisonous plants, but do not replace expert identification.

118. Macrophage Migration Inhibitory Factor as a Novel Biomarker in Acetaminophen Overdose: a Proof-of-Concept Study

Joshua Bloom $^{1}$, Teddy Uzamere ${ }^{2}$, Yasmin L Hurd ${ }^{2}$, Alex F Manini ${ }^{2}$

${ }^{1}$ Mount Sinai St. Luke's-West, New York, NY, USA. ${ }^{2}$ Icahn School of Medicine at Mount Sinai, New York, NY, USA

Background: Acetaminophen overdose is the leading cause of fulminant hepatic failure in the USA. Early prognostication following acute acetaminophen overdose is invaluable in guiding clinical decisions for antidote administration and determination of the need for liver transplant; however, serum acetaminophen and liver transaminases can take hours or days to peak. Macrophage migration inhibitory factor (MIF) is a pleiotropic cytokine and enzyme that has been implicated in early acetaminophen toxicity in animal models, and may prove a useful biomarker in emergency department (ED) patients immediately following an acute acetaminophen overdose.

Hypothesis: We tested the following two hypotheses: (A) MIF is measurable in banked serum specimens from an ED overdose cohort; and (B) MIF is elevated in acetaminophen overdose compared to non-overdose controls.

Methods: Four patients were selected from a prospective cohort of ED patients with suspected acute overdose, with waste specimens allocated for biomarker studies after use for clinical care. Samples from one acetaminophen overdose patient (specimen 1) were compared to two toxicology-proven non-overdose patients without liver failure (negative controls, specimens 2-3) and one non-overdose patient with acute liver failure (positive control, specimen 4). MIF was measured using ELISA kit assays (R\&D Systems).

Results: Negative control specimens 2-3 had MIF levels of 50 and $180 \mathrm{pg} / \mathrm{mL}$, respectively; notably, specimen 3 demonstrated frank hemolysis. Specimen 4 had markedly elevated MIF with $65,000 \mathrm{pg} / \mathrm{mL}$ and ALT of $128 \mathrm{u} / \mathrm{mL}$. The acetaminophen toxicity patient (specimen 1 ) had MIF $1300 \mathrm{pg} / \mathrm{mL}$ and ALT of $19 \mathrm{u} / \mathrm{mL}$.

Conclusion: In this pilot study, MIF is measurable in banked serum specimens from an ED overdose cohort and is elevated in acetaminophen overdose compared to non-acetaminophen controls. Notably, MIF was elevated in a specimen with normal ALT, suggesting utility as an early biomarker of liver toxicity. Measurement of MIF holds promise in guiding early treatment of acetaminophen overdose, and future study is warranted.

119. Alternative Routes of Non-medical Use (NMU) of Prescription Buprenorphine and Fentanyl Patches in the United Kingdom (UK)

Joanna Hockenhull ${ }^{1}$, Paul I Dargan ${ }^{1,2}$, Janetta L Iwanicki ${ }^{3,4}$, Richard C Dart $^{3,4}$, David M Wood ${ }^{1,2}$

${ }^{1}$ Guy's and St Thomas' NHS Foundation Trust, London, UK. ${ }^{2}$ King's College London, London, UK. ${ }^{3}$ Rocky Mountain Poison Center, Denver, CO, USA. ${ }^{4}$ Researched Abuse, Diversion and Addiction Related Surveillance (RADARS) System, Denver, CO, USA

Background: Buprenorphine and fentanyl transdermal patches are widely prescribed and used in the UK. There are reported cases in the USA of opioid patch NMU by non-transdermal routes, such as smoking the patch or injecting the contents. These routes of use have been reported to be associated with severe toxicity and death.
Hypothesis: NMU of prescription buprenorphine and fentanyl transdermal patches by alternative routes is common in the UK.

Methods: Data from second half 2018 UK Survey of Non-Medical Use of Prescription Drugs (NMURx survey (9926 respondents representing 53,370,283 UK adults)) were analyzed to identify respondents reporting last 12-month NMU of buprenorphine or fentanyl patches. The proportion and 95\% CI were calculated for NMU by the intended route (dermal) or by alternative routes (swallowing patch, chewing patch, inhalation by smoking the patch or snorting the contents and/or injection of contents).

Results: The estimated (95\% CI) national prevalence of last 12-month NMU was $0.12 \%(0.07-0.20)$ and $0.09 \%(0.05-0.15)$ for buprenorphine and fentanyl patches, respectively. Dermal application was the most common route of NMU for buprenorphine (71.8\% of those reporting last 12 month NMU, 95\% CI 46.5-88.2); other routes of NMU were included: swallowing (52.6\% (27.2-76.7)), chewing (45.1\% (21.7-70.8)), inhalation (39.7\% (18.0-66.4)), and injection (40.9\% (18.8-67.5)). For fentanyl patches, the most common route of NMU was dermal application $(78.5 \%$ (50.1-93.0)); other routes of NMU were less common than with buprenorphine: swallowing (16.7\% (4.6-45.5)), chewing (19.7\% (5.5$50.6)$ ), inhalation $(18.3 \%(5.1-48.4))$, and injection $(17.1 \%(4.7-46.5))$. Conclusion: NMU of buprenorphine and fentanyl patches in the UK is most commonly by the intended transdermal application. However, swallowing, chewing, injection, and inhaling the patches and/or their contents are significant routes of NMU, particularly for buprenorphine. Understanding NMU by unintended routes will allow appropriate public health messaging about the potential risks of NMU by those routes.

120. Poisonings Due to Storage in a Secondary Container Recorded in the National Poison Data System, 2007-2017

Joseph E Carpenter ${ }^{1,2}$, Brian P Murray ${ }^{3,1}$, Camille A Dunkley ${ }^{1}$, Mark R Layer $^{1}$, Robert J Geller ${ }^{1,2}$

${ }^{1}$ Georgia Poison Center, Atlanta, GA, USA. ${ }^{2}$ Emory University School of Medicine, Atlanta, GA, USA. ${ }^{3}$ Wright-Patterson Medical Center, WrightPatterson AFB, $\mathrm{OH}$, USA

Background: Despite public health messaging and improvements in product labeling, there are sporadic reports of deaths from poisons stored in unlabeled or incorrect secondary containers. There is little published data on this practice outside of case reports.

Research Question: What is the epidemiology of poisoning from substances stored in a secondary container, and what poisons contribute most to morbidity and mortality?

Methods: This is a retrospective analysis of National Poison Data System (NPDS) data. We queried NPDS for all cases associated with the scenario "container transfer involved (product transferred from original container to unlabeled container, incorrectly labeled container, or food container for use or storage and patient accessed product from second container)" from January 1, 2007, to December 31,2017 . We obtained de-identified data for all cases and fatality abstracts for deaths. Data were analyzed for temporal trends, outcome severity, and substances causing severe outcomes. Results are presented descriptively.

Results: There were 45,596 included cases with a known outcome during the study period, increasing from 3224 in 2007 to 5429 in 2017. Children $<6$ years old were identified in 10,112 cases (21.29\%), including four deaths, and $48.39 \%$ of all cases were males. The vast majority $(43,552 ; 95.52 \%)$ resulted in no or minor effects. Of the remaining cases, 1779 (3.90\%) resulted in moderate effects, $242(0.53 \%)$ major effects, and $23(0.05 \%)$ death. Bleach was the most common product identified, followed by hydrogen peroxide, anionic cleaners, alkalis, and laundry detergent. Herbicides and hydrofluoric acid represented $1 \%$ of all cases but resulted in $7 / 23(30 \%)$ and $6 / 23$ (26\%) deaths, respectively. 
Conclusion: Exposure to poisons stored in unlabeled or secondary containers is a common reason for calls to poison centers, yet an uncommon cause of significant injury. Herbicides and hydrofluoric acid represented a disproportionate number of deaths, presenting an opportunity for targeted improvements in product packaging and/or labeling.

\section{Effect of Alkalinization on Elemental Lead Solubility in a Simulated Gastric Environment}

Kartik R Shah ${ }^{1}$, Michael Runyon ${ }^{1}$, Michael C Beuhler ${ }^{2}$

${ }^{1}$ Atrium Health's Carolinas Medical Center, Charlotte, NC, USA. ${ }^{2}$ North Carolina Poison Control, Charlotte, NC, USA

Background: Acute ingestion of elemental lead foreign bodies has resulted in significantly elevated blood lead levels and pediatric deaths. Elemental lead is soluble in an acidic environment but relatively insoluble at alkaline $\mathrm{pH}$. Furthermore, calcium decreases lead absorption by interfering with the lead absorptive receptor.

Hypothesis: Alkalinization of gastric fluid with an oral calciumcontaining agent, such as calcium carbonate, decreases lead solubility, thus reducing the potential for systemic lead absorption and toxicity.

Methods: This is an in vitro randomized controlled study. One freshly cast 3 -week-old $3.3 \mathrm{~g}$ lead sphere ( 00 buckshot) was randomly placed in each of ten tubes containing $20 \mathrm{~mL}$ simulated gastric fluid, with five tubes having $500 \mathrm{mg}$ calcium carbonate (TUMS $®$ ) added at 20 and 140 minutes. Tubes were maintained on a $37{ }^{\circ} \mathrm{C}$ warmed shaker bath. We measured the fluid $\mathrm{pH}$ with $\mathrm{pH}$ paper, and the lead concentrations via inductively coupled plasma spectroscopy, every hour for 4 hours. The total amount of lead liberated was determined at 4 hours in the soluble fraction. We compared the mean amount of lead liberated between the two groups using Student's $t$ test with $p<0.05$ being significant. Results: The $\mathrm{pH}$ of the gastric fluid only tubes remained 1 at every measurement and the $\mathrm{pH}$ of the gastric fluid + calcium carbonate tubes was 6 at every measurement. At hour 4, the total amount of lead liberated in the soluble fraction in the control group vs the calcium carbonate group was 829 vs $13.5 \mathrm{mcg}$ (95\% CI for absolute difference 698-934 mcg; $p=$ 0.0002).

Conclusion: Calcium carbonate antacid alkalinizes gastric fluid $\mathrm{pH}$ well and dramatically decreases the total amount of solubilized lead by 60 fold. This project lends foundational evidence to a low-cost, widely available pre-hospital strategy to decrease lead absorption after unintentional acute lead ingestions.

122. An Acid-Base Model to Simulate Stomach Content pH After Nicotine Ingestion

John A Thompson ${ }^{1,2}$, Todd Ritsema ${ }^{3}$, Robert G Hendrickson ${ }^{1,4}$

${ }^{1}$ Oregon Health \& Science University, Portland, OR, USA. ${ }^{2}$ Oregon Poison Center, Portland, Oregon, USA. ${ }^{3}$ Monterey Peninsula College, Monterey, CA, USA. ${ }^{4}$ Oregon Poison Center, Portland, OR, USA

Background: Nicotine ingestion is increasing in frequency, with widespread use of nicotine containing products, the advent of concentrates, and e-nicotine products. Nicotine is rapidly absorbed in its unprotonated form in an alkaline environment. Absorption is poor in an acidic environment. Nicotine delivery devices are designed with a goal of a basic $\mathrm{pH}$ of the microenvironment at the absorption site. An internet search for nicotine ingestion treatment recommends drinking water. This model sought to explore that recommendation.

Methods: An average stomach acid volume was created using previous studies of stomach contents and $\mathrm{pH}$ in fasting children. This was calculated with average weights based on age. Initial gastric $\mathrm{pH}$ was calculated. Nicotine solution $\mathrm{pH}$ was calculated for different volumes and concentrations. Combining the calculated nicotine and stomach acid resulted in a final $\mathrm{pH}$ and that was compared with the $\mathrm{pH}$ of water. Some results were selected and further calculated with the simulated titration of $255 \mathrm{~mL}$ of phosphoric acid (simulated can of soda).

Results: Large volume and high nicotine concentration ingestions reliably produced a $\mathrm{pH}>7$ in the simulation for a range of gastric acid volumes. Smaller volume ingestions and more dilute nicotine concentrations created more neutral to slightly acidic environments. Adding phosphoric acid to a selection of these could lower the $\mathrm{pH}$ to more acidic ranges.

Conclusion: This simplified model demonstrates concentrated nicotine is able to overwhelm the acid in the stomach. This is dependent on the amount of acid present in the stomach and other factors. Adding water will bring the $\mathrm{pH}$ to but not below 7, arguing against this home remedy recommendation, which may increase the absorption rate of nicotine. Furthermore, while an additional acidic solution may lower the $\mathrm{pH}$ for some ingestions, large volume or concentrated ingestions may not change greatly and more research is required.

\section{Dantrolene Sodium in Neuroleptic Malignant Syndrome: Is} There a Benefit?

Arthur W Daigh ${ }^{1}$, Shawn M Varney ${ }^{1}$, Meiqing Huang ${ }^{2}$, Jonathan A Gelfond $^{1}$, Jeffrey Brent ${ }^{3}$, On Behalf of the ToxIC Investigators Consortium (ToxIC)

${ }^{1}$ University of Texas Health - San Antonio, San Antonio, TX, USA.

${ }^{2}$ University of Connecticut, Storrs, CT, USA. ${ }^{3}$ University of Colorado School of Medicine, Denver, CO, USA

Background: Neuroleptic malignant syndrome (NMS) is a rare condition involving a tetrad of altered mental status, neuromuscular abnormalities, autonomic instability, and hyperthermia after exposure to dopamine antagonists or withdrawal from dopamine agonists. Treatment is stopping the causative agent, aggressive supportive care, and benzodiazepines. Dantrolene sodium, a musculoskeletal relaxant, is sometimes added, but the physiologic basis for its use is unclear. Our aim was to determine in patients with suspected NMS, if adding dantrolene to standard care (supportive care and benzodiazepines) improved clinical outcomes and to describe treatment and outcomes.

Methods: This is a secondary analysis of prospectively collected data of patients diagnosed with NMS recorded in the Toxicology Investigators Consortium Case Registry from the American College of Medical Toxicology from January 1, 2010, to May 31, 2019. We tested for associations with dantrolene administration and patient demographics, symptoms (mental status, neuromuscular, vital signs, and systemic), culprit medications, and other treatments. We created hierarchical clustering heat maps to describe symptom distribution among patients. Associations between categorical and continuous variables were tested using chi-squared and $t$ tests, respectively. Results: Of 131 patients diagnosed with NMS, 95 (72.5\%) were 1965 years old, and $80(61.1 \%)$ were male. Seventeen $(13 \%)$ were treated with dantrolene, $93(71 \%)$ received benzodiazepines, and $31(23.7 \%)$ bromocriptine. There was no association (NS) between dantrolene administration and specific organ system dysfunction, vital sign abnormalities, or any demographic characteristic. The heat map of nervous system symptoms (mental status/neuromuscular abnormalities) suggested three patient clusters corresponding to three levels of severity. Nineteen $(14.5 \%)$ patients were intubated, one cardiac arrest, and zero deaths. Limitations included small sample size, misdiagnosis, and incomplete data on indications for and temporality of dantrolene administration.

Conclusion: We found no association between dantrolene administration and organ system findings in this NMS patient cohort.

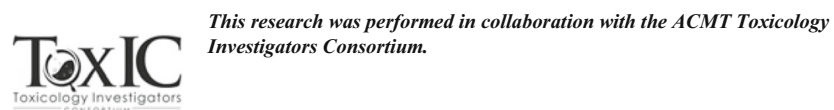


Day 3: Moderated Posters, Abstracts 124-129

124. Agents of Exposure in Transgender Patient Cases Managed by a Toxicologist: an Analysis of the Toxicology Investigator's Consortium (ToxIC) Registry

Mikayla B Hurwitz, Cody R McWhirter, Lexis T Laubach, Gillian A Beauchamp, Judith N Sabino, Beth A Careyva, Matthew D Cook, Marna Rayl Greenberg, Robert D Cannon, Ryan M Surmaitis, On Behalf of the ToxIC Investigators Consortium (ToxIC)

Lehigh Valley Health Network/University of South Florida Morsani College of Medicine, Allentown, PA, USA

Background: The Toxicology Investigators Consortium (ToxIC) database was created in 2010 by the American College of Medical Toxicology (ACMT) to compile data recorded by medical toxicologists. In January 2017, the data field for transgender (and if transgender, male to female or female to male) was added to the ToxIC form. Little is known regarding trends in poisonings within the transgender community.

Research Question: To review medical encounters managed by a bedside toxicologist and provide descriptive data in trends among types of exposures within the transgender demographic.

Methods: In this study, a retrospective ToxIC database review of cases in which the patient identified as transgender was reviewed from January 2017-June 2019 and descriptive demographics were reported.

Results: There were 113 transgender cases included in the ToxIC Registry between January 2017 and June 2019. Among these, 41 $(36.3 \%)$ were male-to-female, $68(60.2 \%)$ were female-to-male, 3 $(2.7 \%)$ were gender nonconforming, and $1(0.9 \%)$ was unknown. Overall, $99(87.6 \%)$ of the poisonings involved pharmaceutical drugs. Regarding agent of exposure, $24(21.2 \%)$ were analgesics; $19(16.8 \%)$ were antidepressants; $10(8.8 \%)$ were antipsychotics, $12(10.6 \%)$ were anti-cholinergic/anti-histamines, $7(6.2 \%)$ were ethanol, $4(3.5 \%)$ were anticonvulsants/mood stabilizers, and 4 $(3.5 \%)$ were cough and cold medications. The most noted abnormal vital sign reported was tachycardia $(n=16,14.2 \%)$. Sixty-one $(54.0 \%)$ of the patients experienced some type of nervous system abnormality, the most common being coma $(36,31.9 \%)$. In this cohort, there were no reported deaths.

Conclusion: Among transgender patients with poisonings reported to the ToxIC Registry, the majority of participants used an analgesic or psychotropic (antidepressant, antipsychotic, anticonvulsant, or mood stabilizer) drug as their agent of exposure. Data describing sex and gender-specific differences in types of exposures/ingestions, as well as outcomes, may inform poisoning prevention practices as well as sex- and gender-based management of patients in this vulnerable population.

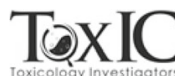

This research was performed in collaboration with the ACMT Toxicolog Investigators Consortium.

\section{How Easy Is It to Find a Clinic Advertising Chelation?}

Adam Blumenberg, Lauren Murphy, John Thompson, Robert G Hendrickson

Oregon Health and Science University, Portland, OR, USA

Background: Approximately 100,000 Americans receive chelation annually. Medical toxicologists manage less than $1 \%$ of cases, and treatment indications may be dubious. Inappropriate chelation is associated with fatalities.

Research Question: How easily may an individual solicit chelation?
Methods: A systematic search strategy was used to detect clinics which advertised chelation directly to the public. In November 2019, www.google.com was queried with the following search string: "intravenous chelation [STATE]" sequentially substituting each state for "[STATE]." Each state's first 100 results were manually assessed. A clinic was included if it (1) advertised chelation services and (2) provided a physical address. If a search result listed multiple clinics, each was evaluated for inclusion. Mobile units, $\mathrm{PO}$ boxes, home infusions, pharmacies, and clinics in a state different than searched were excluded.

Results: Five thousand search results were evaluated yielding 538 clinics meeting criteria. States with 20 or more inclusions were CA 83, AZ 51, FL 45, TX 29, NJ 25, WA 23, and NY 23. States with zero inclusions were IA, KY, ND, SD, and WV. The number of clinics per million state capita is as follows: AL 0.20, AK 4.07, AZ 7.11, AR 1.00, CA 2.10, CO 2.28, CT 3.36, DE 1.03, FL 2.11, GA 1.24, HI 12.0, ID 2.85, IL 0.55, IN 1.05, IA 0.00, KS 1.03, KY 0.00, LA 0.43, ME 4.48, MD 0.66, MA 0.87, MI 1.20, MN 0.71, MS 0.33, MO 1.14, MT 4.71, NE 2.07, NV 1.98, NH 5.16, NJ 2.81, NM 4.30, NY 1.18, NC 1.25, ND 0.00, OH 1.11, OK 2.54, OR 2.62, PA 0.31, RI 0.95, SC 0.79, SD 0.00, TN 0.89, TX 1.01, UT 4.43, VT 1.60, VA 0.82, WA 3.05, WV 0.00, WI 0.69, and WY 3.46. Conclusion: Relative ease of finding advertised chelation services by internet search varies by state.

\section{How Can Artificial Intelligence Help Healthcare Providers} Incorporate Opioid Research into Clinical Practice?

Michael Chary ${ }^{1}$, Michele Marie Burns ${ }^{2}$

${ }^{1}$ Boston Children's Hospital, Boston, MA, USA. ${ }^{2}$ Boston Children's Hospital, Boston, MA, USA

Background: The National Institute on Drug Abuse Clinical Trials Network (NIDA CTN) conducts trials to evaluate, in representative populations, substance abuse treatment interventions that show promise. This proliferation outstrips the ability of healthcare professionals to synthesize research for medical decision-making.

Hypothesis: Machine learning can help healthcare professionals synthesize clinical research into personalized recommendations.

Methods: We created an interactive portal, sudo (substance use disorder ontology), based on NIDA clinical trials related to the treatment of opioid use disorder from 2015 to 2019 . We developed probabilistic logic statements that captured the methods and results of each trial. We evaluated the validity of sudo by querying sudo for 60 clinically relevant questions. A group of board-certified medical toxicologists evaluated the accuracy of sudo's responses to the CTN knowledge base. We evaluated sudo's ability to generate new hypotheses and identify conflicting results by inferring statements that must follow from the logical combination of trial results.

Results: We extracted 38 study reports from NIDA Clinical Data Share, 15 of which explicitly stated results. From those 15 reports, we extracted 161 axioms (77 unique). We extracted $9 \pm 3$ (median \pm interquartile range) axioms from each report. It answered $60 / 60$ competency questions correctly. Sudo, in addition, generated 20 hypotheses based on this knowledge base, 12 of which have related publications indexed in PubMed. Sudo identified conflicts in (1) whether motivational interviewing increased or decreased clinic attendance in adolescents with opioid use disorder and (2) the efficacy of education on HIV and HCV testing.

Conclusion: Probabilistic logic can represent research on the treatment of opioid use disorders transparently. This representation allows physicians to apply medical reasoning at a scale that was not previously possible. In contrast to other artificial intelligence approaches, probabilistic logic can explain its medical decision-making, a crucial requirement for applications used for clinical decisions support. 
127. Educating the Educators: Survey of Medical Toxicology Program Directors

Lesley C Pepin ${ }^{1}$, Jennie Buchanan ${ }^{2,3}$, Anthony Pizon ${ }^{4}$, Matthew Zuckerman $^{5}$

${ }^{I}$ Denver Health Residency in Emergency Medicine, Denver, CO, USA.

${ }^{2}$ Department of Emergency Medicine, Denver Health and Hospital Authority, Denver, CO, USA. ${ }^{3}$ Rocky Mountain Poison \& Drug Center, Denver, CO, USA. ${ }^{4}$ Department of Emergency Medicine, University of Pittsburgh, Pittsburgh, PA, USA. ${ }^{5}$ Department of Emergency Medicine, University of Colorado, Denver, CO, USA

Background: Medical toxicology fellowship program directors are responsible for curriculum design, didactics, bedside education, and graduate medical education assessment. There is a paucity of literature on the educational training of medical toxicology fellowship program directors. While formal faculty development in education is available to directors of other medical specialty programs, it is unclear what resources medical toxicology program directors want, need, or utilize.

Hypothesis: We hypothesized a minority of medical toxicology fellowship directors possess formal medical education training. We also hypothesized fellowship directors are interested in opportunities to garner such experiences. Methods: Utilizing SurveyMonkey, we distributed an anonymous survey to the American College of Medical Toxicology (ACMT) Program Director mailing list to assess whether medical toxicology fellowship program directors engaged in faculty development activities focused on education, as well as their attitudes toward such activities.

Results: Sixty-one percent (17/28) of ACMT fellowship directors responded to the survey as of August 2019. Out of the 17 respondents, $58 \%$ had not participated in faculty development activities focused on education and $75 \%$ had not participated in activities focused on educational theory. The most common activity respondents attended was the ACEP teaching fellowship (4/17). Fifty percent of respondents agreed or strongly agreed knowledge of educational theory is important for medical toxicology fellowship directors. Seventy-five percent of respondents agreed or strongly agreed they would like more opportunities to participate in activities focused on educational theory, practice, and educational administration. Fifty percent of respondents said past faculty development activities helped them in their current roles.

Conclusion: A minority of medical toxicology fellowship program directors possess formal training in educational theory or practices. A significant majority of respondents desire more opportunities for such educational activities. National toxicology organizations should explore planning and implementation of faculty educational development experiences focused on education, educational theory, and educational administration.

128. Characterizing Occupational and Environmental Exposures in the ACMT Toxicology Investigators Consortium Database

Steven J Fishburn, Evan S Schwarz, On Behalf of the ToxIC Investigators Consortium (ToxIC)

Washington University, St Louis, MO, USA

Background: Chemical and physical hazards exist in the workplace that cause significant adverse health effects. These exposures often lead patients to obtain care from medical toxicologists in the clinical setting.

Research Question: We described cases evaluated for occupational or environmental exposures in the outpatient clinical setting.

Methods: We queried the ACMT ToxIC Registry for all cases from the years 2015 to 2016 . We included patients who were 18 years old and were seen in the outpatient/clinic/office setting. We used descriptive statistics to detail demographic data including age and sex, source of referral, reason for the encounter, agent(s) of exposure if known, route of exposure, and treatment. Results: We reviewed 431 cases. Mean age was 48.8 years of age [SD \pm 14 years]. Two hundred fourteen were male (49.6\%). Referrals to clinic were from the primary care provider or other outpatient treating physician $(58 \%)$, self-referral (14\%), employer (14\%), poison center $(8 \%)$, and emergency department (4\%). Two hundred and ninety-two $(67.7 \%)$ patients were identified as having a known exposure and 139 (32.2\%) were listed as having an unknown exposure. Known exposure include metals 62/292 (21.2\%), hydrocarbons 53/292 (18.2\%), gasses/vapors/irritants/ dusts 46/292 (15.8\%), mold 48/292 (16.4\%), caustics 20/292 (6.8\%), insecticides $15 / 292(5.1 \%)$, alcohols $7 / 292(2.3 \%)$, and other household and industrial toxicants 33/292 (11.3\%). Routes of exposure are listed as follows: inhalation $234 / 292(80.1 \%$ ) oral $20 / 292(6.8 \%)$, dermal 22/292 $(7.516 \%)$, and unlisted 16/292 (5.5\%). Antidotal therapy and laboratory evaluations were not obtained in the majority of cases.

Conclusion: Most patients were referred by their primary care doctor and did not require antidotal therapy. This data will help guide future practice in this area of toxicology and will aid with medical toxicology board curriculum.

\section{(2) This research was performed in collaboration with the ACMT}

129. Cancer Mortality Patterns Among Dental Hygienists: a 26 State Death Certificate Study

Muhammed Ershad, James Krueger

Einstein Healthcare Network, Philadelphia, PA, USA

Background: Dental hygienists are exposed to various occupational hazards in the workplace. These include methyl methacrylate; electroplating chemicals; metals such as beryllium, chromium, cobalt, and nickel; and various hydrocarbons. However, there is a scarcity of data in the literature regarding the prevalence of various malignancies among dental hygienists as compared to other occupations.

Hypothesis: Dental hygienists have a higher risk of malignancies as compared to populations in other occupations.

Methods: Data regarding deaths among dental hygienists as compared to other occupations was obtained for the years 1999, 2003-2004, and 2007-2014 for the age group 18 to 90 years from the National Occupational Mortality Surveillance (NOMS) database published by the National Institution for Occupational Safety and Health (NIOSH). NOMS data are collected from death certificates issued by state vital records offices in 26 states in the USA. The risk of various diseases was assessed using proportional mortality ratios (PMR), comparing against the populations from other occupations.

Results: Dental hygienists had a higher risk of being reported to have malignant neoplasms (PMR 133, deaths $(n)=428, p<0.01$, 95\% confidence interval (CI) 120-146), breast cancer (PMR 159, deaths $(n)=87$, $p<0.01$, CI 127-196), malignancies of the female genital organs (PMR $144, n=54, p<0.05$, CI 108-188), and connective tissue and soft tissue malignancies (PMR 357, deaths $(n)=9, p<0.01$, CI 163-677). In the age group of 18 to 64, dental hygienists had a higher risk of leukemia (PMR $229, n=10, p<0.05$, CI 110-422). The PMRs for breast and female reproductive organ malignancies were adjusted for gender.

Conclusion: Dental hygienists could possibly be at a higher risk of acquiring various malignancies including that of the breast and female reproductive tract. Further studies are required to establish a causative relationship.

\section{Day 3: Posters, Abstracts 130-177}

130. An Unknown Pharmacobezoar: Color Identification to the Rescue? 
Eddie C Garcia

University of California, San Francisco, San Francisco, CA, USA

Background: Medical toxicologists are always looking for new ways to determine the identity of unknown ingestions.

Methods: A 17-year-old woman presented to the emergency department 9 hours after the ingestion of a large quantity of unknown pills. Home medications included amphetamine, aripiprazole, cetirizine, fluoxetine, guanfacine, ibuprofen, lamotrigine, lisdexamfetamine, oxcarbazepine, and sertraline. The patient developed cardiogenic shock and pulmonary edema requiring intubation and vasopressor support. A CT of her abdomen revealed a large bezoar in her stomach. A large blue/gray mass of more than 100 pills was removed by endoscopy. We were unable to obtain the bezoar for analysis, but liquid chromatography-mass spectroscopy (LC-MS) analysis of a serum sample was similar to the home medication list. After the fact, we consulted a cosmetic scientist who specializes in color quality control at L'Oreal in an effort to identify the contents of the bezoar. The specialist was sent digital images of the bezoar along with 37 images of various blue medications with instructions to select any number of medications she thought could be represented in the bezoar. All four of the medications she selected were found on the LC-MS results, including the largest LC-MS peak.

Results: Color matching by an expert cosmetic scientist represents a novel method of bezoar or pill fragment identification. PubMed searches for combinations of "color," "bezoar," "pill," and "identification" returned no relevant articles. Mathematically, the color expert had less than a $1 \%$ chance of her degree of accuracy compared to random guessing. The turn-around time for the color matching was less than 20 minutes.

Conclusion: Though color experts are currently largely unavailable to the medical toxicology community, the accuracy displayed in this case and quick turn-around time makes this an interesting future field of study.

\section{Falsely Elevated Digoxin Assay in a Patient Taking Enzalutamide}

Sean Mcann ${ }^{1}$, Merih Tesfazghi ${ }^{2}$, Dinesh Kalra ${ }^{2}$, Henry Swoboda ${ }^{2}$

${ }^{1}$ Toxikon Consortium, Chicago, IL, USA. ${ }^{2}$ Rush University Medical Center, Chicago, IL, USA

Background: Enzalutamaide (trade name Xtandi ${ }^{\circledR}$, Astellas Pharma US, Inc) is a nonsteroidal androgen receptor antagonist indicated in the treatment of metastatic castration-resistant prostate cancer. One previous report describes falsely elevated digoxin concentrations measured by chemiluminescent microparticle immunoassay (CMIA) in a patient taking enzalutamide.

Hypothesis: Enzalutamide may cause false-positive results in quantitative digoxin immunoassays.

Methods: A 64-year-old man with a medical history significant for cardiomyopathy and prostate cancer on digoxin and enzalutamide was referred to the emergency department (ED) with an elevated digoxin level of $9.8 \mathrm{ng} / \mathrm{mL}$ (approximately 30 hours after last digoxin administration). His digoxin dose had recently been decreased from $250 \mu \mathrm{g}$ daily to $125 \mu \mathrm{g}$ three times weekly following a previous elevated digoxin concentration. The patient denied any unusual symptoms. Electrocardiogram demonstrated normal sinus rhythm at a rate of 82 beats per minute with no digoxin effect. Laboratory evaluation was also significant for serum potassium of $3.7 \mathrm{mmol} / \mathrm{L}$ and serum creatinine of $0.78 \mathrm{mg} / \mathrm{dL}$. The incongruity between the measured digoxin concentration and the remainder of the patient's clinical presentation and laboratory values prompted suspicion of laboratory error. The same specimen was aliquoted and sent to other laboratories for digoxin measurement by five alternative methodologies.

Results: Digoxin concentration was within or below the therapeutic range as measured by DGNA Flex® (Siemens Dimension), ECLIA ${ }^{\circledR}$ (Roche Elecsys), DRI ${ }^{\circledR}$ (Thermo Fisher), EMIT 2000® (Beckman Coulter), and liquid chromatography tandem mass spectrometry. The hospital laboratory, utilizing CMIA ${ }^{\circledR}$ (Abbott Architect), reported supratherapeutic concentrations. The manufacturer was informed and independently verified the interference.

Conclusion: Digoxin concentrations measured by CMIA may be falsely elevated in patients taking enzalutamide; other quantitation methods did not demonstrate this interference.

\section{Inadvertent Administration of Intraperitoneal Penicillin G Benzathine}

Haylee Veazey ${ }^{1}$, Andrew Laudenbach ${ }^{1}$, Travis D Olives ${ }^{1,2}$

${ }^{1}$ Hennepin Healthcare, Minneapolis, MN, USA. ${ }^{2}$ Minnesota Poison Control Center, Minneapolis, MN, USA

Background: Penicillin $\mathrm{G}$ benzathine is an injectable suspension of the classic beta-lactam antibiotic. It is typically administered intramuscularly to treat susceptible infections such as streptococcal pharyngitis and syphilis. When given intramuscularly, non-allergic side effects are rare. When given intraperitoneally, the effects are not described.

Hypothesis: Intraperitoneal administration of penicillin $\mathrm{G}$ benzathine presents with symptoms of peritonitis.

Methods: This is a single patient chart review. A 29-year-old female with a history of seizure disorder presented to the emergency department with severe abdominal pain. The pain started immediately following an attempted ventrogluteal injection of penicillin $\mathrm{G}$ benzathine 1.2 million units at an urgent care facility to treat streptococcal pharyngitis diagnosed earlier the same day. On arrival, the abdomen was markedly tender with rebound and guarding. She was evaluated with abdominopelvic CT scan and routine laboratory examinations.

Results: Laboratory workup was remarkable for leukocytosis $(16.33 \times 10$ cells $/ \mathrm{mm}^{3}$ ) with neutrophil predominance. Abdominopelvic CT scan with IV contrast revealed a small amount of subdiaphragmatic free air and a small amount of fluid along the inferior margin of the liver at the hepatic flexure of the colon, interpreted as related to perforation of the right hemicolon. The patient was treated with broad spectrum antibiotics and intravenous analgesia and admitted to a general surgery service for serial abdominal exams. Symptoms gradually improved, leukocytosis abated, and she was discharged on hospital day 3 without surgical intervention. Chart review revealed no additional visits for related problems over the subsequent 6 months.

Conclusion: Inadvertent intraperitoneal injection of penicillin $\mathrm{G}$ benzathine is not described in the literature. In this case, a good outcome resulted with conservative treatment, suggesting that peritonitis resulting from inadvertent intraperitoneal penicillin $\mathrm{G}$ benzathine does not mandate laparotomy. When the ventrogluteal approach is chosen for intramuscular injection, care should be taken to avoid this rare complication.

\section{Anticipated Maximal Peak and Post-distribution Sodium} Concentrations After Rapid Infusion

Adam Blumenberg, John Thompson, Lauren Murphy, Robert G Hendrickson

Oregon Health and Science University, Portland, OR, USA

Background: Treatment of poisoning with tricyclic antidepressant or another drug with sodium channel blockade may require rapid infusion of sodium-containing solutions. Dosing is often empiric rather than targeting a particular serum concentration. Peak concentrations are rapidly attenuated by tissue distribution.

Research Question: What is the maximum expected peak serum concentration of sodium after rapid infusion?

Methods: To model the immediate post-infusion serum sodium concentrations, a one-compartment model of an adult patient with $3 \mathrm{~L}$ of plasma was generated. To model post-distribution sodium concentration, a onecompartment model of an adult patient with $14 \mathrm{~L}$ of extracellular fluid was generated. Initial serum sodium concentrations were modeled using 
low to high range $(105-165 \mathrm{mEq} / \mathrm{L})$. Estimated sodium concentrations after 1-3 respective boluses of $50 \mathrm{~mL} 8.4 \%$ sodium bicarbonate and $100 \mathrm{~mL}$ of $3 \%$ sodium chloride were calculated. The formula used for calculations was as follows: ([Sodium] * CompartmentVolume + AddedSodium) / (CompartmentVolume + FluidVolume).

Results: Each $50 \mathrm{~mL}$ bolus of $8.4 \%$ sodium bicarbonate $(50 \mathrm{mEq} \mathrm{Na})$ is expected to transiently raise serum sodium concentration by up to $13.7-$ $14.2 \mathrm{mEq} / \mathrm{L}$. After distribution, each $50 \mathrm{~mL}$ bolus of $8.4 \%$ sodium bicarbonate $(50 \mathrm{mEq} \mathrm{Na})$ is expected to raise serum sodium concentration by up to $3.0-3.2 \mathrm{mEq} / \mathrm{L}$. Each $100 \mathrm{~mL}$ bolus of $3 \%$ sodium chloride $(51.3 \mathrm{mEq} \mathrm{Na})$ is expected to transiently raise serum sodium concentration by up to $10.5-14.5 \mathrm{mEq} / \mathrm{L}$. After distribution, each $100 \mathrm{~mL}$ bolus of $3 \%$ sodium chloride $(51.3 \mathrm{mEq} \mathrm{Na})$ is expected to raise serum sodium concentration by up to $2.4-3.2 \mathrm{mEq} / \mathrm{L}$.

Conclusion: Maximum peak post-infusion sodium concentrations may be estimated in a one-compartment model.

\section{Quality Measures for Medical Toxicology Patients: an 18-Month Update}

Anthony F Pizon ${ }^{1}$, Paul Wax ${ }^{2}$, Sharan Campleman ${ }^{2}$, Dana Karshenas ${ }^{2}$, Jeffrey Brent ${ }^{3}$, On Behalf of the ToxIC Investigators Consortium (ToxIC) ${ }^{1}$ University of Pittsburgh School of Medicine, Pittsburgh, PA, USA.

${ }^{2}$ American College of Medical Toxicology, Phoenix, AZ, USA.

${ }^{3}$ University of Colorado School of Medicine, Denver, CO, USA

Background: Beginning in January 2018, the American College of Medical Toxicology (ACMT) initiated a program to collect quality measure data on medical toxicology patients. Utilizing the Toxicology Investigators Consortium (ToxIC) infrastructure, a Qualified Clinical Data Registry (QCDR) was established to provide the opportunity for participating sites to voluntarily report on six quality measures that were previously developed under the ACMT Quality Measure Initiative.

Hypothesis: Participation in a quality strategy for medical toxicologists through voluntary reporting in the Toxic Investigators Consortium Registry (ToxIC) can facilitate the development of benchmarks in medical toxicology treatment.

Methods: Over 18 months, data were collected on performance measures on the following topics: opioid misuse screening, pregnancy testing in poisoned patients, timely EKG assessment in drug ingestions, appropriate treatment of acetaminophen ingestions, assessment of suspected toxic alcohol exposures, and repeat assessment of salicylate concentrations in salicylate ingestions. The measured performance did not necessarily reflect the practice of the medical toxicologist since the care may have occurred prior to the medical toxicologist consultation (e.g., ordering of EKG after emergency medicine presentation); hence, the measures reflect the multidisciplinary care of the medical toxicology patient.

Results: Forty-nine medical toxicologists from 17 ToxIC sites participated in the ToxIC QCDR over 18 months. Performance rates were as follows: opioid screening $51.7 \%$, pregnancy testing $74.6 \%$, EKG assessment $54.3 \%$, appropriate treatment of acetaminophen ingestions $14.7 \%$, toxic alcohol assessment $36.7 \%$, and serial salicylate determinations $23.1 \%$.

Conclusion: Performance rates are considerably lower on medical toxicology patient performance measures than ideal benchmarks established by the Centers for Medicare and Medicaid Services (>95\% performance). Other specialties have already demonstrated that variability in healthcare delivery is not optimal for patient outcomes. Our data suggest medical toxicologists must continue to improve the quality and consistency of healthcare delivery to the poisoned patient.

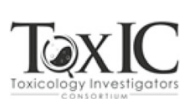

This research was performed in collaboration with the ACMT Toxicology Investigators Consortium.

\section{Poisoning Data in Developing Nations: a Systematic Review}

Brian Kaiser, Blase Ferraris, Harsh Sule, Lewis Nelson, Cynthia Santos Rutgers New Jersey Medical School, Newark, NJ, USA

Background: In the USA, epidemiological poisoning information is available through large databases such as the National Poison Data System and the Centers for Disease Control and Prevention. In developing nations, similar programs are often not yet firmly established.

Research Question: What current literature and aggregate population data from developing countries are available regarding poisonings and deaths due to poisonings?

Methods: Following PRISMA methodology, a literature search was performed using the United Nations (UN) list of least developed countries during the years 2013-2017. A literature search was performed on two databases: PubMed and Scopus. Only studies that contained numerical data on poisoning incidence and mortality were included.

Results: Of the 510 publications reviewed, 26 met the criteria for inclusion. In total, there were 11,779 poisonings and 5042 mortalities from 26 different nations in this data set. The average mortality was $7.5 \%$. Nepal had the most publications (30.8\%). Among the substances reported were aluminum phosphide, benzodiazepines, bongkrekic acid, botulism, carbamates, grayanotoxin, household products, hydrocarbons, kerosene, lead, mushrooms, organophosphates, pesticides, pharmaceuticals, rat poisons, snakebites, and tetrodotoxin. The largest number of poisonings were from snakebites $(48.8 \%)$ and organophosphates (13.8\%). Singlecenter studies were $61.5 \%$, regional multicenter studies were $34.6 \%$, and one study (3.8\%) was a national multicenter study.

Discussion: Nearly half of all reported poisonings in this literature search were from snakebites, reflecting the World Health Organization (WHO) formally listing snakebite envenomation as a highest priority neglected tropical disease in June 2017. Some countries were overrepresented. Nepal, for example, represented almost one third of the included publications, while other nations did not have any relevant, published data. There is a scarcity of nationwide-published data on poisonings.

Conclusion: Further research is needed to better characterize the incidence and impact of poisonings and mortality in developing nations.

\section{Management Trends in the Toxicology Investigators Consortium Registry: a 10-Year Review}

Diane P Calello ${ }^{1,2}$, Kim Aldy ${ }^{3}$, Sharan Campleman ${ }^{4}$, Paul Wax, ${ }^{3,4}$, Jeffrey Brent ${ }^{5}$, On Behalf of the ToxIC Investigators Consortium (ToxIC) ${ }^{1}$ Rutgers New Jersey Medical School, Newark, NJ, USA. ${ }^{2}$ New Jersey Poison Information and Education System, Newark, NJ, USA. ${ }^{3}$ University of Texas Southwestern Medical Center, Dallas, TX, USA. ${ }^{4}$ American College of Medical Toxicology, Phoenix, AZ, USA. ${ }^{5}$ University of Colorado School of Medicine, Denver, CO, USA

Background: Management of poisoned patients may change over time due to new information about efficacy and adverse effects and shifting practice patterns among medical toxicologists. The ToxIC Registry, a multicenter prospective database of patients cared for at the bedside by medical toxicologists, was developed in 2010 and has nearly 10 years of data.

Research Question: How has management by medical toxicologists changed over time?

Methods: ToxIC Annual Reports were searched from 2010 to 2019 regarding three categories: antidotes, chelators, and GI decontamination. Chelators and decontamination methods were reported from 2012 to 2019. Percentages were compared from year to year, which reflect the percentage of patients given a specific therapy among those in whom an entry was made in that category.

Results: Antidotes: The most common antidotes were $N$-acetylcysteine (average $29.4 \%$ of all antidotes, range 27-32.9), naloxone (19.3\%, 17-21.9), and sodium bicarbonate $(11.9 \%, 10.9-14)$ with minimal variation over 10 years. Other infrequently used antidotes demonstrated minimal change, including fomepizole $(3 \%, 2.8-4)$, hyperinsulinemia-euglycemia $(0.9 \%, 0-1.4)$, and 
lipid emulsion $(0.66 \%, 0-1.2)$. The one antidote which showed steady decline in use was physostigmine, $(6.4 \%, 2.3-11)$ which was reported in $9 \%$ of antidote cases in 2010 , peaked in 2012, and steadily declined to $3.6 \%$ in 2018. Chelators: DMSA and DFO were most common each year, followed by EDTA and BAL. Penicillamine and DMPS were rarely reported. GI decontamination methods: Of patients receiving GI decontamination, activated charcoal was most common $(80 \%, 78.8-81)$, followed by whole bowel irrigation (11\%, 7.7-14.2) and gastric lavage (3\%, 1.4-6).

Conclusion: Practice patterns were stable over the study period. This may reflect areas of consensus among board-certified medical toxicologists. Several therapies which are the subject of much study were rarely used in the registry, such as hyperinsulinemia and lipid emulsion. Future study may analyze behaviors and attitudes among medical toxicologists about specific therapies.

\section{ToxIC}

This research was performed in collaboration with the ACMT Toxicology Investigators Consortium.

\section{Validation of a Rules-Based Algorithm for Toxidrome Recognition}

Daniel Nogee ${ }^{1}$, Adrian Haimovich ${ }^{1}$, Katherine Hart ${ }^{2}$, Anthony Tomassoni ${ }^{1}$ ${ }^{1}$ Department of Emergency Medicine, Yale School of Medicine, New Haven, CT, USA. ${ }^{2}$ Connecticut Poison Control Center, Farmington, CT, US

Background: Rules-based software algorithms are becoming increasingly prevalent in medicine. These are programs designed by human subject experts that utilize patient data to determine disease states in an automated fashion using decision trees or variable weighting of inputs. Such systems may prove useful for recognition of toxidromes at levels ranging from individual patients to national trends. However, there is little available information on their validity with real patient data.

Research Question: Can retrospective data from the National Poison Data System (NPDS) database be used to validate the accuracy of a rules-based algorithm for toxidrome recognition?

Methods: A previously designed rules-based algorithm produced ranked differential diagnoses for NPDS-reported cases of human exposures to single substances in 1 of 15 toxicant classes resulting in moderate to major effects or death in Connecticut in 2018.

Results: Of 476 included cases with at least one algorithm-recognized clinical feature, the algorithm correctly identified the exposure substance as first-ranked in 243 cases (51.1\%), within the top 3 ranks (of 15) for 362 cases (76.1\%), and within the top 5 for 389 cases (81.7\%). Of 262 cases with at least three recognized clinical features, the algorithm correctly ranked $170(64.9 \%)$ first, $213(81.3 \%)$ in the top 3 ranks, and 229 $(87.4 \%)$ in the top 5. Algorithm accuracy varied by toxicant: opioid exposures were well recognized (158 of 249 (63.5\%) cases correctly ranked first and $222(89.2 \%)$ in top 5 ranks) while alcohol exposures were poorly recognized ( 2 of 46 (4.3\%) cases of ethylene glycol, ethanol, or methanol correctly ranked first, 17 (37.0\%) in top 5 ranks).

Conclusion: NPDS data were useful in assessing the real-world accuracy of this algorithm, providing a benchmark for other rules- or artificial intelligence-based algorithms. Further work is needed to determine the effects of providing additional clinical features or different toxicants on algorithm accuracy.

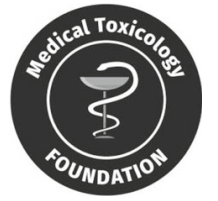

This research was supported by the 2019-2020 Medical Toxicology Foundation / Emergency Medicine Foundation Research Award.
138. A Novel Hand-Powered Device to Deliver Mechanical Closed Chest Compressions for Small Rodent Cardiac Arrest Research

Jason B Hack ${ }^{1}$, Peter R Chai ${ }^{2}$

${ }^{1}$ Brown University, Providence, RI, USA. ${ }^{2}$ Brigham and Women's Hospital, Boston, MA, USA

Background: Rodent (rat, mouse) cardiac arrest models have become more common over the past decade. These animals allow for larger numbers and increased potential impact when compared to large animal studies in some circumstances. CPR research using these models requires either sustained rapid manual compression ( $>200$ beats per minute (BPM)) or costly, commercially produced devices to provide this. Both options have limitationsvariability in compression and fatigue vs. expense.

Methods: This was an uncontrolled, descriptive, IACUC-approved convenience sample substudy. We designed, fabricated with approved materials, a simple and cost-effective $(<\$ 800)$, hand-powered mechanical device to deliver high-frequency CPR to rats. Two anesthetized $250 \mathrm{~g}$ rats with venous and arterial catheters were given intravenous cocaine to induce cardiac arrest. After asystole, two providers cycled between manual and mechanical closed chest compressions to generate sustained 200 BPM and consistent mean arterial pressure. Rat 1/provider 1: 4 one-minute manual cycles then 4 one-minute machine cycles; rat 2/ provider 2: the order of procedure was reversed.

Results: The manual 200-BPM compression goal and mean arterial pressure varied more than mechanical compressions - evidenced by slowing and MAP fall off. Manual compression resulted in significant fatigue, discomfort, and limited duration of effective compressions compared to mechanical delivered CPR.

Conclusion: This low-cost, hand-cranked, novel device provided improved consistency in BPM, MAP with less much less operator fatigue when compared with manual compression. This device also has the potential to facilitate cardiovascular arrest research in small animals performed in resource-limited environments.

139. Introduction of Hack's Impairment Index (HII Score) Mobile Device App into Nursing Protocol Does not Interfere with Task Completion

Jason B Hack, Janette Baird, Dennis Ferrante

Brown University, Providence, RI, USA

Background: Almost 35 million alcohol impaired (AI) patients present to US EDs annually. There is no standard assessment. The HII score is a clinical tool designed to score a standardized, serializable evaluation of AI patients. In our center, nurses routinely perform HII scores using paper prompts; scores are repeated PRN. We sought to determine if the introduction of a mobile device HII score app would affect bedside performance of assessment.

Methods: This chart review QI project compared the number, repetitions, and completeness of HII score documentation performed by nurses on $\mathrm{AI}$ ED patients over 6.5 months using two data gathering techniques: group 1: paper-based HII scores for 90 days prior to intervention; group 2: iPadbased HII scores for 90 days after intervention. There was a 2 -week period for staff training and app feedback between intervals.

Results: Group 1: 476 AI ED patients were seen and had HII scores ordered; $339(71.2 \% ; 95 \% \mathrm{CI} \pm 4.1 \%)$ patients had $\mathrm{HII}$ assessments with a total of 539 HII scores documented. An average of 1.60 (SE \pm 0.01$)$ serial assessments occurred per patient. Five $(1.1 \%$; $95 \% \mathrm{CI} \pm 1.1 \%)$ scores were incomplete. Group 2: 569 AI ED patients were seen and had HII scores ordered; $420(73.8 \%$; 95\% CI $\pm 3.6 \%)$ patients had HII assessments with a total of $639 \mathrm{HII}$ scores documented. An average of $1.52(\mathrm{SE} \pm 0.03)$ serial assessments occurred per patient. Four $(0.9 \%$ : $(95 \% \mathrm{CI} \pm 0.09 \%)$ had incomplete HII scores. Interviews with nurses revealed "quicker" and "preferred" use of the electronic format. Limitations included one center, chart review, not directly observed. 
Conclusion: The HII score mobile device app did not interfere with nursing performance, repetition, or completeness of assessment of AI ED patients. App format was preferred by nurses which may translate into better assessments.

\section{Acceptance and Usability of a Novel Telemedicine Platform to} Enhance Post-overdose Care in the Emergency Department

Jeffrey T Lai, Brittany P Chapman, Stephanie P Carreiro, Amy D Costigan, Karla M Rodriguez-Perez, Gerardo Gonzalez, Kavita M Babu University of Massachusetts Medical School, Worcester, MA, USA

Background: Emergency departments (EDs) are the primary sites of medical care after near-fatal opioid overdose but are poorly equipped to provide adequate substance use treatment planning prior to discharge. Limited access to clinicians trained in addiction medicine and behavioral health, particularly in rural locales, exacerbates this issue. We developed a telemedicine protocol to facilitate timely access to substance use disorder evaluations (SUDE) for people who use drugs (PWUD).

Hypothesis: A telemedicine-based behavioral health intervention (teleSUDE) is acceptable to PWUD and feasible for use in the ED setting. Methods: This was a single arm, prospective observational study of a convenience sample of participants presenting to the ED after a known or suspected opioid overdose. Participants received their SUDE via the telemedicine interface and subsequently completed a brief survey regarding participant attitudes toward and acceptance of the teleSUDE platform.

Results: Twenty-seven individuals were approached to participate. Twenty consented to participate in our pilot study, and seven declined. Sixteen participants responded to the post-encounter survey regarding teleSUDE's usability and acceptability. The technology was favorably reviewed by all study participants, and participants were unanimous in rating the technology as easy to use and found the audio and video quality to be satisfactory. The majority reported that they thought teleSUDE felt similar to having an in-person encounter.

Conclusion: A low-intensity, affordable telemedicine platform is a feasible and acceptable way to enhance referrals to addiction treatment from the ED. This pilot study demonstrates that (1) PWUD are receptive to using a telemedicine platform to conduct SUDE and (2) the technology platform is a viable solution to enhance the availability of substance use disorder counseling and treatment in patients presenting for evaluation after near-fatal overdose.

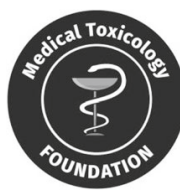

This research was supported by the 2018 Medical Toxicology Foundation Practice Award.

141. Applications of Machine Learning Within Clinical Toxicology: a Review

Nikolaus Matsler ${ }^{1}$, Christopher Hoyte ${ }^{2}$, Benjamin Hatten ${ }^{2}$

${ }^{1}$ Denver Health, Denver, CO, USA. ${ }^{2}$ University of Colorado Health, Aurora, CO, USA

Background: Machine learning (ML), as a field, has seen tremendous advancement over the last decade. Algorithms and applications within ML, such as neural networks (NN), have been applied to many different fields within medicine in myriad capacities. The potential applications of ML in medical toxicology, however, are not well described. Thus, this review aims to elucidate the application of ML principles to the field of medical toxicology, specifically clinical applications, and serve to foster discussion regarding potential future applications.

Methods: A narrative literature search was performed utilizing the keywords "machine learning," "neural network," and "toxicology." Within the broad definitions of medical toxicology and ML, papers were then selected for their relevance based on direct relation to eventual human clinical application.

Results: Eight papers were selected and included for examination and analysis. Seven of the eight papers utilize ML to predict the impact of potentially toxic compounds (novel or known) on human or environmental systems, while the final paper is a review of ML techniques related to toxicology; however, no papers were found relating directly to clinical (bedside) practice in medical toxicology.

Conclusion: Currently, ML applications to medical toxicology appear limited to laboratory research and focus primarily on predicting toxicological side effects of existing and novel compounds and treatments. While other medical disciplines are embracing and applying ML to more directly clinical applications, there exists room for growth in this area for medical toxicology. While rigorous validation and caution will still be needed, this avenue may provide new tools to the bedside clinician to improve patient outcomes.

142. Crotalus horridus Envenomation Complicated by Persistent Thrombocytopenia Despite Antivenin Therapy with Crotalidae Polyvalent Immune Fab and Crotalidae Immune F(ab')2

Shirley Shao ${ }^{1}$, Ryan T Marino ${ }^{2,3}$, Andrew F Micciche ${ }^{1}$, Alexander M Sidlak ${ }^{1}$, Rachael C Westover ${ }^{1}$, Joseph H Yanta ${ }^{1}$, Michael G Abesamis ${ }^{1}$, Anthony F Pizon ${ }^{1}$

${ }^{1}$ University of Pittsburgh School of Medicine, Pittsburgh, PA, USA.

${ }^{2}$ University Hospitals, Cleveland, OH, USA. ${ }^{3}$ Case Western Reserve School of Medicine, Cleveland, OH, USA

Background: Crotalus horridus is a venomous pit viper endemic to eastern North America known for causing tissue toxicity and hematologic derangements. It also causes profound thrombocytopenia likely due to crotalocytin, a unique venom component. Although Crotalidae polyvalent immune fab (CroFab®) has been utilized for treatment of all pit viper envenomations in the USA, recurrent coagulopathy has occurred in approximately half of patients studied. In a prospective trial in 2015 , Crotalidae immune $F\left(a^{\prime}\right) 2$ (Anavip ${ }^{\circledR}$ ) was shown to reduce the risk of delayed coagulopathy. However, it is unclear if Anavip ${ }^{\circledR}$ will reverse delayed thrombocytopenia.

Hypothesis: Anavip ${ }^{\circledR}$ will not reverse late thrombocytopenia in C. horridus envenomation.

Methods: This is a single patient case report. A previously healthy 33-year-old man was envenomed by a Crotalus horridus in his left hand. He received 18 vials of $\mathrm{CroFab} \circledast$ for thrombocytopenia $\left(61,000 / \mathrm{mm}^{3}\right)$ and hand swelling. His platelets improved to $273,000 / \mathrm{mm}^{3}$ with a fibrinogen of $92 \mathrm{mg} / \mathrm{dL}$. His thromboelastogram revealed alpha 53 degrees and MA $53 \mathrm{~mm}$. Fibrinogen normalized on day 2 to $252 \mathrm{mg} / \mathrm{dL}$ and remained stable. On day 4 , his platelets decreased to $3000 / \mathrm{mm}^{3}$. His thromboelastogram demonstrated a decrease in alpha to 36 degrees and MA to $18 \mathrm{~mm}$. He was then given 10 vials of Anavip ${ }^{\circledR}$. His platelets made no improvement $\left(4000 / \mathrm{mm}^{3}\right)$, and he was monitored in the hospital for bleeding. The patient developed bloody bowel movements on day 9, and his hemoglobin declined from 14.7 to $9.7 \mathrm{~g} / \mathrm{dL}$; however, his bleeding was self-limited, and he did not require blood product transfusion. His thrombocytopenia persisted to day of discharge on day $12\left(19,000 / \mathrm{mm}^{3}\right)$.

Conclusion: Crotalus horridus envenomations cause late thrombocytopenia likely related to crotalocytin. Despite treatment with both $\mathrm{CroFab} \circledast$ and Anavip ${ }^{\circledR}$, our patient developed persistent thrombocytopenia as well as bleeding complications. Administration of Anavip ${ }^{\circledR}$ does not reverse late thrombocytopenia from $C$. horridus envenomation.

143. Delayed Onset Hemotoxicity Following Crotaline Envenomation with Delayed Response to Treatment with $\mathbf{F}\left(\mathbf{a b}^{\prime}\right) 2$ Antivenin

Han T Gao, Bryan M Corbett, Binh T Ly University of California San Diego, San Diego, CA, USA

Background: North American crotaline envenomation is characterized by thrombocytopenia, coagulopathy, and hypofibrinogenemia that is usually treated with Fab antivenin. However, $30-53 \%$ of cases have been 
shown to present with recurrence or delayed hemotoxicity. These cases can be resistant to treatment, even with redosing of Fab antivenin.

Hypothesis: Some cases of delayed hemotoxicity may be difficult to treat, even with administration of the new $\mathrm{F}\left(\mathrm{ab}^{\prime}\right)_{2}$ antivenin.

Methods: This is a single patient case report. A 7-year-old female presented with pain and swelling after crotaline envenomation to her right foot. She was brought to the emergency department (ED) and given four vials of Fab antivenin with improvement in symptoms. She had no signs of hemotoxicity and was discharged after 24 hours. Six days later, the patient had follow-up testing and her mother was told the results were above the upper limit of detection.

Results: At discharge, her results were PT $12.1 \mathrm{~s} \mathrm{(reference} \mathrm{11.5-14.3} \mathrm{s),}$ aPTT $30 \mathrm{~s}$ (reference $24-37 \mathrm{~s}$ ), and fibrinogen $311 \mathrm{mg} / \mathrm{dL}$ (reference 160 $425 \mathrm{mg} / \mathrm{dL}$ ). She was brought back to the ED and given five vials of F(ab $')_{2}$ antivenin. Testing performed after the infusion showed no change in hemotoxicity with PT $>120 \mathrm{~s}$, aPTT $>130 \mathrm{~s}$, and fibrinogen $<45 \mathrm{mg} / \mathrm{dL}$. Another five vials of $\mathrm{F}\left(\mathrm{ab}^{\prime}\right)_{2}$ antivenin was given, and repeat tests showed improvement with PT $19.9 \mathrm{~s}$ and aPTT $29 \mathrm{~s}$. However, she remained hypofibrinogenemic at $<45 \mathrm{mg} / \mathrm{dL}$. Since the patient had no evidence of bleeding and appeared to have a delayed treatment response, it was decided to continue observation. After 24 hours, repeat testing showed fibrinogen had improved to $155 \mathrm{mg} / \mathrm{dL}$. She was discharged with close follow-up.

Conclusion: Crotaline envenomation with delayed onset hemotoxicity may be slow to improve even after treatment with $\mathrm{F}\left(\mathrm{ab}^{\prime}\right)_{2}$ antivenin.

\section{Severe Recurrent Coagulopathy and Thrombocytopenia in a} Pediatric Patient Following Crotaline Envenomation

\author{
Jacob A Lebin ${ }^{1,2}$, Kathy T Vo ${ }^{1,2}$, Craig G Smollin ${ }^{1,2}$ \\ ${ }^{1}$ University of California, San Francisco, San Francisco, CA, USA. \\ ${ }^{2}$ California Poison Control System, San Francisco Division, San \\ Francisco, CA, USA
}

Background: Recurrence of coagulopathy and thrombocytopenia after initial treatment with Crotalidae polyvalent immune Fab (FabAV) is common. Late bleeding complications are a feared, but rare, occurrence in patients with severe hematologic venom effects. Despite this, the most effective treatment for severe recurrent coagulopathy remains uncertain. We describe a case of a pediatric patient with severe recurrent coagulopathy and thrombocytopenia following crotaline envenomation that was managed with FabAV alone.

Methods: This is a single patient chart review.

Results: A 3-year-old girl presented to the emergency department following a rattlesnake envenomation to the right ankle. Her initial labs were as follows: platelets 260 , INR 1.0, and fibrinogen $210 \mathrm{mg} / \mathrm{dL}$. She received four vials of FabAV and was discharged the following day. Five days after treatment with FabAV, she returned to the emergency department for evaluation of new bruising. Her repeat labs were as follows: platelets 20, INR 3.3, and undetectable fibrinogen. She received four additional vials of FabAV with worsening of her coagulopathy (INR 8.7, fibrinogen undetectable). She had no evidence of clinically significant bleeding. The patient was given eight additional vials of FabAV with rapid improvement in her coagulopathy and thrombocytopenia over 4 hours and she was discharged 2 days later. There are few reports of pediatric patients with severe hematologic venom effects following crotaline envenomation successfully managed with FabAV alone. Pediatric patients may be at higher risk for recurrent coagulopathy due to higher renal blood flow and increased clearance of unbound FabAV. While the mechanism of recurrent coagulopathy is unknown, administration of blood products in the setting of unneutralized venom may be ineffective. This case illustrates the rapid improvement of severe coagulopathy after additional antivenom, without blood product administration.

Conclusion: Severe recurrent coagulopathy and thrombocytopenia following crotaline envenomation can be successfully managed with FabAV alone.

145. Crotalidae Immune $\left.F\left(a^{\prime}\right)^{\prime}\right)$ Administration Is Associated with Few Adverse Reactions

Priya Srihari, Richard F Clark

University of California, San Diego, San Diego, CA, USA

Background: Crotalidae immune $\mathrm{F}\left(\mathrm{ab}^{\prime}\right)_{2}$, or Anavip, is an equinederived antivenom for rattlesnake envenomation that was FDA approved and became available in October 2018. Previous studies have reported mild adverse effects associated with administration, including pruritus, nausea and/or vomiting, arthralgias, myalgias, and headache.

Hypothesis: Further reports of Crotalidae immune $\mathrm{F}\left(\mathrm{ab}^{\prime}\right)_{2}$ administration for rattlesnake envenomation continue to demonstrate few adverse reactions.

Methods: This is a retrospective case series. Data was pulled from the California Poison Control System between April and September of 2019. Results: Twenty-five cases of rattlesnake envenomation were reported. Seven received only Crotalidae immune $\mathrm{F}\left(\mathrm{ab}^{\prime}\right)_{2}$ as treatment. One patient reported one of the known adverse reactions, headache, and this occurred more than 36 hours after administration of antivenom. All other patients reported no adverse reactions. There were also three patients who first received Crotalidae polyvalent immune $\mathrm{Fab}(\mathrm{CroFab})$ followed by $\mathrm{F}(\mathrm{ab}$ $\left.{ }^{\prime}\right)_{2}$. One patient had a reaction of pruritus and lip swelling to Fab treatment and was treated with solumedrol, diphenhydramine, and epinephrine. A test dose of $\mathrm{F}\left(\mathrm{ab}^{\prime}\right)_{2}$ was given, and the patient became tachycardic and reported anxiety and dyspnea. She was treated with lorazepam, solumedrol, diphenhydramine, and albuterol. The other two patients reported no adverse reactions. Previous studies have demonstrated mild adverse reactions to Crotalidae immune $\mathrm{F}\left(\mathrm{ab}^{\prime}\right)_{2}$; serious adverse effects were infrequent and thought unrelated to antivenom administration. Since FDA approval of the $\mathrm{F}\left(\mathrm{ab}^{\prime}\right)_{2}$ antivenom, many hospitals have begun to stock this new antivenom. Here we report an additional case series of patients receiving $\mathrm{F}\left(\mathrm{ab}^{\prime}\right)_{2}$ antivenom with few adverse effects.

Conclusion: A review of recent cases of rattlesnake envenomations reported to the California Poison Control System and treated with Crotalidae immune $\mathrm{F}\left(\mathrm{ab}^{\prime}\right)_{2}$ showed few adverse reactions. Treatments appear to continue to be safe.

146. Neurotoxicity from North American Rattlesnake Envenomation Reversed by Crotalidae Immune $\mathrm{F}\left(\mathbf{a b}^{\prime}\right) 2$

Erik S Fisher ${ }^{1,2}$, Michelle Ruha ${ }^{3,4}$

${ }^{1}$ Prisma Health-Upstate, Greenville, SC, USA. ${ }^{2}$ University of South Carolina School of Medicine Greenville, Greenville, SC, USA. ${ }^{3}$ Banner - University Medical Center Phoenix, Phoenix, AZ, USA. ${ }^{4}$ University of Arizona College of Medicine - Phoenix, Phoenix, AZ, USA

Background: Neurotoxicity is an uncommon manifestation of North American pit viper envenomations and affects just over $5 \%$ of patients. Reports of the efficacy of Crotalidae polyvalent immune fab (CroFab®) in reversing neurotoxicity are mixed. No previous case reports exist using newly available Crotalidae immune $\mathrm{F}\left(\mathrm{ab}^{\prime}\right) 2$ (Anavip ${ }^{\circledR}$ ) in patients manifesting neurotoxicity.

Methods: This is a single patient case report.

Results: A 20-year-old man with a history of asthma and seizures was walking in central Arizona when he felt a pain in his left shin. He did not see a snake or hear a rattle. Within minutes, he developed chest pressure without palpitations, wheezing, or dyspnea. He was bitten by a Western Diamondback (Crotalus atrox) 2 years prior but did not require antivenom. He arrived at an emergency department 1 hour later. He was afebrile, with BP $137 / 79$, HR 88, and oxygen saturation $98 \%$ on room air. Exam revealed two small, tender puncture marks on the anterolateral left lower leg. The leg was otherwise nontender. The remainder of his exam was unremarkable. In the ED, he developed involuntary, intermittent contractions of the left lateral thigh muscles. The contractions were externally 
visible, but the patient did not feel them. Initial laboratory studies including platelet count, prothrombin time, and creatine kinase were within normal ranges. No fibrinogen testing was available. On arrival to our center, he reported perioral paresthesias in addition to chest pressure. He developed mild swelling extending 2-3 cm from the bite. The lower extremity muscle contractions persisted and became painful. Ten vials of Anavip ${ }^{\circledR}$ were administered. Within 20 minutes of infusion start, his muscle contractions, chest pressure, and perioral paresthesias completely resolved. Symptoms did not return during overnight observation. Conclusion: This is the first report of North American rattlesnake venom-induced neurotoxicity reversed by Anavip®. The presence of neurotoxicity should not deter clinicians from administering Anavip®.

\section{Evolution of Thromboelastogram After Timber Rattlesnake Envenomation}

Rachael C Westover, Andrew F Micciche, Shirley Shao, Alexander M Sidlak, Anthony F Pizon

University of Pittsburgh, Pittsburgh, PA, USA

Background: Serial platelet and fibrinogen levels are often obtained after crotalid envenomation to determine level of coagulopathy. However, numerous studies have demonstrated that thromboelastograms may better identify early or delayed coagulopathy.

Hypothesis: Thromboelastogram (TEG) abnormalities precede platelet and fibrinogen changes, serving as an earlier marker for development of coagulopathy.

Methods: This is a single patient chart review. A 66-year-old man presented after timber rattlesnake (Crotalus horridus) envenomation. He was initially evaluated at an outside hospital and found to have thrombocytopenia (2000/mcL), INR elevation (1.56), and hypotension (80/49 $\mathrm{mmHg}) . \mathrm{He}$ was given six vials of Crotalidae polyvalent immune Fab (ovine) and transferred to a tertiary care center for further management. Due to worsening pain and swelling, the patient received 10 vials of Crotalidae immune $F\left(a^{\prime}\right) 2$ (equine) and was admitted. Serial TEG and laboratory studies were obtained prior to and after $\mathrm{F}\left(\mathrm{ab}^{\prime}\right) 2$ antivenom administration.

Results: One pre-antivenom and four post-antivenom TEGs (days 0, 1, 3, 5) with corresponding laboratory values were obtained. On presentation, alpha-TEG, MA-TEG, and k-value TEG were abnormal with platelet count of $182,000 / \mathrm{mcL}$ and fibrinogen $<20$. Ma-TEG values decreased as thrombocytopenia developed but rebounded faster than serum platelet count. Alpha-TEG values continued to fall until a significant rebound on day 3 with a corresponding fibrinogen level of 264 . K-value was significantly elevated on presentation and was unable to be obtained on days 0 , 1 , and 3 despite increasing fibrinogen levels. On day 5 , fibrinogen was 323 and platelets 27,000 with normal alpha-TEG and improving MATEG and $\mathrm{k}$-value TEG.

Conclusion: Thromboelastogram showed evidence of platelet dysfunction prior to development of thrombocytopenia, and k-value TEG still demonstrated evidence of dysfunction after serum fibrinogen level normalization. Thromboelastogram may serve as a more clinically relevant marker for coagulopathy than serum platelet and fibrinogen levels after timber rattlesnake envenomation.

148. Global Snake Envenomation in Our Own Backyard: Exotic Venomous Snakebites in Pennsylvania Reported to PA Poison Centers

Steve W Miller ${ }^{1}$, Amanda Korenoski ${ }^{2}$, Ian Gereg ${ }^{3}$, Kevin C Osterhoudt ${ }^{1,4}$ ${ }^{I}$ The Poison Control Center at Children's Hospital of Philadelphia, Philadelphia, PA, USA. ${ }^{2}$ Pittsburgh Poison Center, Pittsburgh, PA, USA. ${ }^{3}$ The Philadelphia Zoo, Philadelphia, PA, USA. ${ }^{4}$ Center for Global Health at Children's Hospital of Philadelphia, Philadelphia, PA, USA

Background: The global burden of snakebite is $>400,000$ long-lasting disabilities and $>100,000$ deaths annually. Antivenom is on The WHO's
Essential Medicines List, and snakebite has been classified as a highpriority neglected tropical disease. Pennsylvania is home to three native venomous pit-vipers, but international trade in venomous snakes can also lead to envenomation from globally notorious venomous snakes locally. Research Question: What is the descriptive epidemiology of non-native snake envenomation in PA?

Methods: In this descriptive, retrospective cohort, the American Association of Poison Control Centers' National Poison Data System was searched using generic codes 013700 and $013702-013710$ to identify calls to PA's two regional poison control centers involving non-native venomous snakebite from 2004 through 2018. IRB-exempt de-identified records were abstracted.

Results: PA poison centers received 1452 human exposure calls coded as snakebites during the study period, including 847 coded as venomous, and 19 ultimately identified as non-native venomous snakebites. The age range of victims of exotic snake envenomation was 16 to 63 years; $84 \%$ were male. The three most commonly implicated snakes were cobras (genus Naja) and vipers (Trimeresurus sp. and Atheris sp.). A "dry bite" was noted in 1/19 cases, while $53 \%$ were categorized as "moderate" or "severe"; there were no deaths. Antivenom was administered to eight snakebite victims, procured from the Philadelphia zoo in four cases and from other zoos in the remaining cases.

Conclusion: Venomous snakebite is a significant, though neglected, global health burden worldwide. Bites from "exotic," non-native, venomous snakes occur in Pennsylvania with 19 reported to PA poison centers over a 15 -year period. Poison center expertise and the stocking of global snake antivenins by zoos provide a valuable public health safety net. This descriptive cohort is limited by voluntary reporting and information bias; significant miscoding was noted in the database.

\section{Idiopathic Intracranial Hypertension in Confirmed Case of Systemic Loxoscelism}

Sam J Wagner, Brandon J Ricke, Tony N Rianprakaisang, Stephen L Thornton

University of Kansas Health System, Kansas City, KS, USA

Background: Systemic loxoscelism secondary to brown recluse spider envenomation is known to cause hemolysis, coagulopathy, and shock; however, there is currently no reported association with elevated intracranial pressures.

Methods: This is a single patient chart review.

Results: A 10-year-old female with cystic fibrosis presented with a 2-day history of abdominal pain, vomiting, fever, and diffuse rash. A necrotic ulcer was found on her back suspicious for brown recluse envenomation. The patient became hypotensive, received fluids and broad-spectrum antibiotics including doxycycline for suspected septic shock, and was transferred to an academic medical center. She was found to have hemolysis and DIC (undetectable haptoglobin, hemoglobin 7.8, LDH 864, INR 2.0, PTT 37.5, platelets 78) as well as rhabdomyolysis (CPK 7393). The patient received supportive care for presumed systemic loxoscelism with subsequent resolution of hematologic disturbance on day 4. All antibiotics were discontinued on day 6 . However, that same day, she developed diplopia, CN VI palsy, and papilledema with subsequent confirmation of idiopathic intracranial hypertension (IIH) following lumbar puncture. Acetazolamide was started, and she experienced improvement in vision symptoms. On day 10, ELISA testing was positive for brown recluse envenomation. The patient was discharged in stable condition.

Conclusion: To our knowledge, this is the first reported case of IIH associated with systemic loxoscelism. The patient did receive nine doses of doxycycline, which is known to cause intracranial hypertension; however, the timeline is inconsistent with many previous cases of tetracyclineinduced IIH which develops over weeks to months. This patient had rapid development of symptoms over days raising the possibility that her IIH may have been related to her brown recluse envenomation. We report a case of IIH associated with systemic loxoscelism. While confounding 
factors exist in this case, toxicologists should be made aware that IIH is a potential complication of brown recluse envenomation.

\section{A Case Series of Critical Illness Following Palytoxin Exposure}

Jason M Devgun ${ }^{1}$, Ian J Holley ${ }^{2}$, Michael E Mullins ${ }^{1}$

${ }^{1}$ Washington University School of Medicine, St. Louis, MO, USA.

${ }^{2}$ Barnes-Jewish Hospital, St. Louis, MO, USA

Background: Palytoxin is a marine toxin found in zoanthid corals that inhibits the sodium-potassium ATPase pump. Critical illness from inhalational exposure is rare and hypotension requiring vasopressor support is not well described.

Methods: This is a chart review of four patients. Patient 1, a 47-year-old male presented to the emergency department (ED) 5 hours after removing zoanthid corals from four rocks with a torch. The rocks were brought indoors and scrubbed under hot water in the kitchen where his family was eating lunch: patient 2 (40-year-old female), patient 3 (15-year-old male), and patient 4 (12-year-old female). The family presented to the ED 5-7 hours postexposure. Patient 3 was in the home for 5 hours preceding presentation.

Results: All patients presented with respiratory irritation, dyspnea, fever, and myalgias. All had tachycardia, tachypnea, and leukocytosis (ranging $15.8 \times 10^{9}-44.4 \times 10^{9} / \mathrm{L}$ ). Patient 1 was hypertensive upon presentation. $\mathrm{He}$ developed fever, hypoxia requiring $4 \mathrm{~L}$ nasal cannula oxygen (NC$\mathrm{O}_{2}$ ), and saline (NS)-responsive hypotension (nadir 78/42 $\mathrm{mmHg}$ ) requiring ICU care. He was discharged hospital day 4 . Patient 2 presented with hypertension. She was admitted to the ICU for tachypnea and developed delayed hypoxia requiring 2-3 $\mathrm{L} \mathrm{NC}-\mathrm{O}_{2}$ with bilateral interstitial opacities on CXR on day 2. She was discharged hospital day 5. Patient 3 was hypotensive, febrile, and ill-appearing and transferred to a tertiary care center. He received $4 \mathrm{~L}$ of NS and was placed on an epinephrine infusion. $\mathrm{He}$ briefly required nonrebreather oxygen and remained on $\mathrm{NC}-\mathrm{O}_{2}$ through day 2 with a right lower opacity on CXR. He required vasopressor support intermittently through day 3 and was discharged on hospital day 4. Patient 4 presented with hypertension and fever. She developed NS-responsive hypotension but did not require oxygen and was discharged on hospital day 2.

Conclusion: Inhalational exposures to palytoxin can lead to hypotension requiring vasopressor support and ICU-level care.

151. Environmental Toxic Exposures Using Companion Animals as an Indicator of Human Toxicity: a Case Report and Literature Review

Andrew C Beck, Erica M Lash, Jason B Hack

Brown University, Providence, RI, USA

Background: Years ago, it was recognized that animals could be used as a tool to indicate toxic environments - e.g., coal miners using canaries. Animal physiology demonstrated symptoms before co-exposed humans were injured. Occasionally, diagnosis of cryptic toxic exposures can be aided by history of pet health.

Methods: This is a case report and literature review. Briefly, a 55-yearold man presented to an ED with 2 weeks of progressive shortness of breath, altered mental status, and unsteady gait. There were no other symptoms. While his examination, laboratory data, and chest radiograph suggested pneumonia, he had unexplained persistent confusion, ataxia, and incoordination despite investigation. In the ICU, his partner gave more history - while no one else was sick at home, three healthy pet cats had unexpectedly died within the week. This prompted further exploration, which revealed he'd been "melting dental fillings to extract silver" on the home stove. Mercury testing revealed toxic levels: serum (396 ng/ $\mathrm{mL}$ (normal 0-9 $\mathrm{ng} / \mathrm{mL}$ )) and urine $(322 \mathrm{ng} / \mathrm{mL}$ (normal 0-20 ng/mL)). Testing of his family revealed elevated $\mathrm{Hg}$ levels. All were chelated and the EPA condemned the home. Literature search (1967-2019) identifying publications involving "companion animals," "human toxicity," and related terms was performed in PubMed, Canary Database, and other online resources.

Results: Seven hundred and forty-eight papers met initial search criteria; 25 were ultimately included for analysis. These reports describe, support, and/or recommend obtaining companion animal (dogs, cats, and birds) health history as clues to elucidate and direct human toxic exposure investigation for acute and chronic exposures. Examples include mercury, lead, Teflon, herbicide, and asbestos.

Conclusion: Companion animals, when co-exposed to environmental toxic substances, mount symptoms and signs in advance, and often more demonstrably, than humans. There is strong literature support that questions about pet health should be a part of initial focused toxicologic history gathering for suspected environmental exposures.

\section{The Caterpillars Are Coming!}

Elizabeth T Jacobs ${ }^{1}$, Diana J Dean ${ }^{1,2}$, Cynthia K Aaron ${ }^{1}$, Andrew M King $^{1,3}$, Eric R Malone ${ }^{1,2}$

${ }^{1}$ Michigan Poison Center, Detroit, MI, USA. ${ }^{2}$ Henry Ford Hospital, Detroit, MI, USA. ${ }^{3}$ Detroit Medical Center, Detroit, MI, USA

Background: Every year, caterpillars come out in droves to feast and prepare for the transformation into butterflies and moths. This occurs from spring to autumn. Caterpillars appear defenseless but that is not the case. There are thousands of different species in Michigan alone. A few species have urticating hair and a few have venom-containing hairs similar to hypodermic needles. The result of contact with these defense mechanisms includes local itching, erythema, and edema. Absolute numbers of caterpillars depend on environmental factors such as food source and predators. The Michigan Poison Center has seen an increase in the calls related to caterpillar exposures over the past 14 years.

Methods: This is a retrospective chart review of the Michigan Poison Center data on calls for caterpillar exposure from 2005 to 2019. Yearly totals were tabulated.

Results: Over the 14 years through 2019, there has been an increase in the calls related to caterpillar exposure with 2019 being the highest at 55 calls. Year 2005 had 13 calls followed by a 7-year lull with call volume ranging from 3 to 8 per year. Call volume began increasing yearly starting in 2014. Though most calls do not contain adequate descriptors to speciate, they report minor, local effects on the hands or arms consistent with caterpillar dermatitis. Serious cases included those that had generalized urticaria as well as those who had oral contact with edema involving the upper airway.

Conclusion: As climate changes and human encroachment of habitats continue, wildlife exposure frequency changes as well.

\section{Accidental Arthrotomy Causing Aseptic Monoarthritis Due to Agave Sap}

Sam T Ontiveros, Alicia B Minns

University of California San Diego, San Diego, CA, USA

Background: Agave species has previously been reported to cause both vesicopapular and purpuric dermatitis. Causative agents are believed to be steroidal saponins and calcium oxalate crystals in agave sap. Aseptic inflammatory arthritis has been reported from foreign bodies such as thorns or cactus needles after inadvertent arthrotomy; however, this has not been described from agave plants.

Hypothesis: Agave sap irritants may cause an aseptic inflammatory arthritis mimicking a septic arthritis.

Methods: This is a single patient chart review. A 27-year-old male presented with malaise, left knee pain, swelling, and inability to range his knee 2 hours after suffering an accidental stab wound to his left lateral knee by an agave plant spine. The patient had no history of intravenous drug use, sexually transmitted infections, or current genitourinary complaints. Workup included complete blood count, basic metabolic panel, 
ESR, CRP, lactate, blood cultures, gonorrhea/chlamydia testing, synovial fluid studies, and surgical washout.

Results: The patient remained afebrile with normal vital signs. WBC was 14 $\left(1000 / \mathrm{mm}^{3}\right)$, ESR was $2 \mathrm{~mm} / \mathrm{h}$, CRP was $2.2 \mathrm{mg} / \mathrm{dL}$, and lactate was $2.6 \mathrm{mmol} / \mathrm{L}$. The remainder of his $\mathrm{CBC}$ and BMP was within normal limits. Synovial fluid WBC was $92,730\left(1000 / \mathrm{mm}^{3}\right)$ with $75 \%$ neutrophils and no crystals. Gram stain was negative. After repeating synovial fluid testing, orthopedics took the patient for a washout remarkable for turbid fluid and no foreign bodies. Synovial fluid and blood cultures remained without growth. Antibiotics were stopped on POD 1 and the patient was discharged from the orthopedic service. At 2 weeks follow-up, he is doing well.

Conclusion: Agave sap toxicity may contribute to aseptic inflammatory arthritis seen acutely in the emergency department.

154. Thujone Oil-Induced Convulsions: a Homeopathic Misadventure

Sam T Ontiveros, Shaun D Carstairs

University of California San Diego, San Diego, CA, USA

Background: There is an increasing interest in "natural" and homeopathic therapies. Many commercially available essential oils contain little to none of their active ingredient, while others contain far higher concentrations than advertised.

Hypothesis: Administration of unexpectedly potent thujone preparations may precipitate serious adverse effects, including convulsions.

Methods: This is a single patient chart review. A 10-month-old female with a recent 5-day history of upper respiratory congestion presented to an emergency department (ED) after multiple convulsions. The patient's grandmother reported that she routinely administered "thuja" extract to her children and grandchildren for upper respiratory symptoms by placing the thuja solution diluted with olive oil under the tongue or intranasally; she reported that such solutions are used in Russia to treat large adenoids. Shortly after intranasal administration, the patient began convulsing and developed apnea with cyanosis. Following spontaneous resolution of convulsions, the grandmother then manually induced vomiting while the child was obtunded. The child then had multiple recurrent convulsions during EMS transport and in the ED. The patient underwent endotracheal intubation and was administered midazolam before transitioning to propofol and fentanyl prior to transfer to a regional children's hospital. Results: The patient had no further convulsions and was extubated on hospital day 2. Chest X-ray was remarkable for mild consolidation in the right upper lobe concerning for aspiration, but the patient's oxygen saturation, temperature, work of breathing, and white blood cell count remained within normal limits. The patient continued to do well and was discharged home on hospital day 3 .

Conclusion: Thujone-containing solutions are readily available and can induce serious adverse effects, including convulsions, when administered to pediatric patients.

155. An Atypical Violent and Intoxicated Patient: a Case of Severe Thiamine Deficiency and Lactatemia

Robert C Pueringer, Annie M Arens, Jon B Cole Minnesota Poison Control Center, Minneapolis, MN, USA

Background: Thiamine deficiency (TD) is an uncommon cause of lactic acidosis (LA) and prompt treatment with high-dose IV thiamine is crucial. Methods: This is a case report of a 26-year-old male with schizophrenia and alcohol use disorder found stumbling around downtown. EMS reported hypoglycemia ( $28 \mathrm{mg} / \mathrm{dL})$, ataxia, and weeks of heavy drinking. In the ED, he was combative, tachycardic $(130 \mathrm{~s})$, and hypothermic $\left(34.4{ }^{\circ} \mathrm{C}\right)$. He received $15 \mathrm{mg}$ droperidol and $10 \mathrm{mg}$ midazolam for agitation. Initial labs showed lactatemia over $12 \mathrm{mmol} / \mathrm{L}$, acidemia (pH 7.14), normal electrolytes, anion gap 20, and relatively low betahydroxybutyrate (BHB) $0.67 \mathrm{mmol} / \mathrm{L}$. Blood glucose normalized with dextrose. Toxicological workup was negative. There was no seizure activity, post-ictal state, or access to antihyperglycemics. Family confirmed months of progressive psychotic behavior with substance abuse, ataxia, memory problems, and weight loss. His LA and acidemia persisted despite receiving $5 \mathrm{~L}$ of IV fluid. Six hours after $500 \mathrm{mg}$ IV thiamine administration, his LA decreased dramatically to $2.5 \mathrm{mmol} / \mathrm{L}$. Admission serum thiamine level returned at $80 \mathrm{nmol} / \mathrm{L}$ (normal 70-180). Results: In this case, an intoxicated, violent patient turned out to have critical illness that could have resulted in catastrophic neurological injury without timely treatment. TD is rarely seen in the developing world, and we considered it only because of his history, low BHB-to-lactate ratio, and persistent LA after fluid resuscitation. Improvement in LA 6 hours after IV thiamine and Wernicke-like symptoms supported the diagnosis. Serum thiamine levels have little clinical correlation and low sensitivity for TD.

Conclusion: TD is uncommon and can present similarly to the common violent intoxicated patient, and delayed treatment has potentially disastrous consequences. TD should be considered when LA persists after fluid resuscitation with a low BHB-to-lactate ratio in the setting of chronic alcohol use, starvation/malabsorption, or psychiatric disease.

156. Concentrated Acidic Ingestions Are Associated with Increased Morbidity and Mortality when Compared to Concentrated Nonacidic/Alkali Caustic Ingestions

Wells J Brambl ${ }^{1,2}$, Natasa Simonovska ${ }^{3}$, Danil Petrovski ${ }^{3}$, Silvija P Gottesman $^{4}$, Daniela Chaparoska ${ }^{3}$

${ }^{1}$ Maimonides Medical Center, Brooklyn, NY, USA. ${ }^{2}$ Northwell Health, Manhasset, NY, USA. ${ }^{3}$ Mother Teresa Hospital Center for Toxicology Skopje, Skopje, Greater Skopje, Macedonia, the former Yugoslav Republic of. ${ }^{4}$ Ackerman Academy of Dermatopathology, Manhattan, $N Y$, USA

Background: Caustic ingestions are an important cause of mortality and morbidity and represent a significant burden to healthcare systems. Regulations that ensure the safety of household cleaning supplies vary regionally. Skopje, North Macedonia, offers a unique window into caustic ingestions because of the high volume of caustic ingestion, availability of in-department endoscopy, and standard practice regarding documentation of the product ingested.

Hypothesis: Acidic caustic ingestions are associated with higher rates of mortality.

Methods: We performed a retrospective chart review on de-identified charts of discharged or deceased patients from a January 2018 to May 2019 at Mother Theresa Hospital, Department of Medical Toxicology. The EMR was queried for diagnosis code of corrosive ingestions ICD t54 for patients 18 and older who were admitted to the Toxicologic Intensive Care Unit.

Results: Sixty-four female and 35 male charts met inclusion criteria ( $p=$ 0.0046). There was no difference in the average age between sexes. Ninetytwo were intentional ingestions and 16 were unintentional. Forty-seven had an acidic exposure and 52 ingested an alkaline bleach product or other household cleaning agent. Twenty-one percent of the acidic group died vs $0 \%$ of the alkali/other group $(p=0.0002)$. There was no significant difference in mortality between male and female subgroups. On average, patients with acidic ingestions spent 18 days hospitalized compared with 6 days in the alkali/other group $(p<0.0001)$. Patients with acidic ingestions were more likely to have Zargar grade $2 \mathrm{~b}$ or higher injuries when compared to the alkali/other group $(p<0.0001)$. Finally, $26 \%$ of patients in the acidic group were documented to eventually develop strictures when compared to $8 \%$ of the alkali/other group $(p=0.0383)$.

Conclusion: Ingestions of concentrated acid products (e.g., $18 \% \mathrm{HCl}$ ) are associated with increased mortality, longer hospital stay, worse mucosal injury, and increased development of strictures. 
157. Emergency Mushroom Identification Through Social Media: Comparison with Verified Expert Opinion

\author{
Abigail F Kerns ${ }^{1}$, Cecily Franklin ${ }^{2}$, Matthew P Stripp \\ ${ }^{1}$ Department of Emergency Medicine, Allegheny Health Network, \\ Pittsburgh, PA, USA. ${ }^{2}$ Western Pennsylvania Mushroom Club, \\ Pittsburgh, PA, USA
}

Background: Identification has not historically been achieved in 7595\% of mushroom exposures. Social media groups are already providing assistance for rapid mushroom identification and potential guidance for medical decision-making. This study evaluates posts in one prominent Facebook group and compares identifications to those provided by verified expert mycologists.

Methods: A quantitative evaluation was performed of a public emergency mushroom identification Facebook group. Administrators assist with identification following exposures to guide decision-making. Other than administrator status, identifiers may not have verified credentials. A post will typically include photos, location, and environment of the mushroom(s). The most recent 30 posts from October 2019 involving human mushroom ingestions were collected and compared to a second group of experts provided by a regional mycology society. Digital slideshows were created with the same information from the posts. A third expert served as a tiebreaker in the event the two experts disagreed. Comparison between groups was performed. A positive identification was defined as any administrator proposing a matching answer to the verified expert identification. If the social media group failed to propose a matching identification, this was considered a negative identification. Complete lack of identification was considered positive if matching between groups.

Results: A total of 30 Facebook posts were analyzed, with 27 resulting in a positive identification $(90 \%)$. For positive identifications, the average time between initial post and positive identification was 4.6 minutes.

Conclusion: Social media may provide timely and accessible information regarding a mushroom identification. Caution should be taken when medical advice is provided by social media and the accuracy of this guidance was not addressed in this study. More extensive study is warranted on a larger scale. Higher quality details about the mushrooms could increase accuracy of identification.

158. Nephrotoxic Mushroom Poisonings: Epidemiology, Toxidromes, Treatments, and Outcomes

James H. Diaz

LSU Health Sciences Center, New Orleans, LA, USA

Background: Mushroom poisonings are increasing worldwide today as adolescents mistake poisonous mushrooms for psychedelic ones and recent immigrants mistake poisonous mushrooms for native edible ones. Since mushroom poisonings are increasing following ingestions of known, newly described, and formerly edible species, the objectives of this study are to identify regional nephrotoxic mushroom species, to design a toxidromic approach to earlier diagnoses based on onset times of renal insufficiency, and to compare the outcomes of renal replacement strategies.

Methods: This is a convenience sample survey that queried several internet search engines with keywords to identify case reports, case series, and surveillance studies on nephrotoxic mushroom poisonings and their treatments during the study period, 1957-present. Keywords included mushrooms, poisonous, nephrotoxic, myotoxic; orellanus syndrome, orellanine; and rhabdomyolysis, mushroom-induced. Article exclusion criteria included opinion-editorial and perspective articles.

Results: Although hepatotoxic amatoxin-containing mushrooms cause most mushroom poisonings and fatalities, nephrotoxic mushrooms, most commonly Cortinarius species, can cause acute renal insufficiency and failure. Recently, several new species and even formerly edible species of nephrotoxic mushrooms have been identified including Amanita proxima and Tricholoma equestre in Europe, Amanita smithiana in the USA and
Canada, Amanita pseudoporphyria in Japan, Amanita punctata in Korea, and Russula subnigricans in China. Renal replacement therapies including temporary hemodialysis are often indicated in the management of nephrotoxic mushroom poisonings with renal transplantation reserved for extracorporeal treatment failures.

Conclusion: Unlike the outcomes of amatoxic mushroom poisonings which are often fatal without liver transplantation, nephrotoxic mushroom poisonings that are diagnosed early and managed with temporary renal replacement therapies have uniformly good outcomes with full recovery of pre-existing renal function. Renal transplantation should not be recommended too early as partial to complete recovery of normal renal function has occurred even after months of hemodialysis.

159. A Case Report of Defatting Dermatitis Requiring Surgical Intervention from a Prolonged Dermal Methylene Chloride Exposure

John M Rague ${ }^{1}$, Lesley Pepin ${ }^{2}$, Jennie Buchanan ${ }^{1}$

${ }^{1}$ Rocky Mountain Poison and Drug Safety, Denver, CO, USA. ${ }^{2}$ Denver Health Medical Center, Denver, CO, USA

Background: Methylene chloride exposure is commonly associated with carbon monoxide poisoning. Although not well-defined in the literature, methylene chloride can also cause a significant defatting dermatitis and full thickness dermal injury from prolonged dermal exposure as demonstrated in this case report.

Methods: This is a single patient chart review. A 37-year-old male, nonsmoker, with no past medical history presented to the emergency department with bilateral hand pain, swelling, and erythema after cleaning a spilled container of paint and epoxy remover (60\% methylene chloride, $20 \%$ methanol, $<5 \%$ petroleum) with ungloved hands. Estimated time of dermal exposure was 45 minutes. Additionally, a database search was conducted on methylene chloride and dermal exposure using the MEDLINE/PubMed database.

Results: On a hospital day (HD) 0 , the patient was transferred to a tertiary burn center for definitive management of dermal chemical injury and was noted to have a carboxyhemoglobin $(\mathrm{COHb})$ percentage of $2.4 \%$. On hospital day 1 , repeat $\mathrm{COHb}$ was $1 \%$. Wound care consisted of oat beta glucan and xeroform, and the patient was found to have nonviable epidermis. On HD 3, the patient was taken to the operating room (OR) for debridement of full thickness injury to the bilateral palms, total body surface area (TBSA) of $2.5 \%$. The debrided palms were dressed with a bioactive tissue allograft composed of dehydrated human amnion/chorion membrane. On postoperative day 5 , the dressings were taken down and dressed with bacitracin and xeroform with pain control achieved with oral analgesics agents. The patient was discharged from the burn center with scheduled follow-up.

Conclusion: Methylene chloride exposure is associated with the risk of developing carbon monoxide poisoning. It is important to also consider prolonged dermal exposure and the risk of significant skin injury necessitating surgical intervention.

160. Cardiac Glycoside Toxicity After Ingestion of a Single PongPong Seed in a Nonindigenous Region

John M Rague ${ }^{1}$, Laurie S Halmo ${ }^{1}$, Kennon Heard ${ }^{2}$

${ }^{1}$ Rocky Mountain Poison Drug and Safety, Denver, CO, USA. ${ }^{2}$ University of Colorado, Aurora, CO, USA

Background: Cerbera odollam, commonly known as the pong tree or suicide tree, is a tree indigenous to areas of India and Southeast Asia and is a common plant poisoning in this region. The fruit of the tree contains a kernel, known as a pong-pong seed, which contains a number of cardiac glycosides and poisoning is uncommon in Western countries. With the use of the internet, poisoning from Cerbera odollam is now increasingly being seen in Western countries. 
Methods: This is single patient case report. A 43-year-old male with a past medical history of human immunodeficiency virus presented to the emergency department (ED) for persistent nausea and vomiting. The patient reported ingestion of a single pong-pong seed purchased on the internet in an attempt to end his life approximately 48 hours prior to presentation; he ultimately sought care because of persistent nausea and vomiting and lightheadedness.

Results: The patient's initial vital signs were unremarkable, although his heart rate was noted to fluctuate between 50 and 68 beats per minute. His basic metabolic panel was notable for a potassium of $3.8 \mathrm{mEq} / \mathrm{L}$ and a digoxin concentration of $0.4 \mathrm{ng} / \mathrm{mL}$. The electrocardiogram (ECG) demonstrated sinus bradycardia at 58 beats per minute and ST segment scooping. The patient was observed in the ED and provided with intravenous fluid hydration and anti-emetics. Serial basic metabolic panels and ECGs were obtained and no abnormalities were noted. The patient improved symptomatically and was admitted to an inpatient psychiatric facility. Although confirmatory testing for cerberin was not performed, the patient's history, profuse nausea and vomiting, bradycardia, ECG changes, and detectable digoxin concentration all are consistent with ingestion of Cerbera odollam.

Conclusion: Although exposures are rare in the USA, pong-pong seeds are available for purchase on the internet and physicians should be aware of their digoxin-like toxicity.

\section{Case Report of Severe Hemlock Poisoning}

Anastasia V Markovtsova ${ }^{1}$, Anita $\mathrm{Ma}^{2}$, Eddie Garcia ${ }^{3}$

${ }^{1}$ Stanford University, Stanford, CA, USA. ${ }^{2}$ California Poison Control System, San Francisco, CA, USA. ${ }^{3}$ University of California San Francisco, San Francisco, CA, USA

Background: Conium maculatum, or poison hemlock, is a deadly, coniinecontaining plant that resembles a number of edible plants. Death can occur with ingestion of as little as $6 \mathrm{~g}$ of leaves. Despite being a common species, severe toxicity from C. maculatum is exceedingly rare.

Methods: A 53-year-old woman was brought to the emergency department (ED) in cardiac arrest. She had foraged and eaten a meal of what her family believed to be sweet fennel. One hour later, she was weak and had rapid, shallow breathing. Once paramedics arrived, she was apneic and bradycardic. Intubation was difficult due to copious vomiting. The ED team was able to restore spontaneous circulation, but the patient had minimal brain stem reflexes and subsequent imaging showed cerebral edema. One month after the ingestion, the patient showed no functional improvement, was placed on hospice care, and was lost to follow-up.

Results: Sweet fennel, or Foeniculum vulgare, looks similar to C. maculatum. Our patient displayed symptoms consistent with severe coniine poisoning: rapidly progressive weakness, respiratory paralysis, and bradycardia. Three professional botanists were consulted to identify the foraged plant. None of them believed that the photographs of the plant in question were of C. maculatum due to the shape of the leaves and the lack of purple spots on the stems. However, field research conducted at a later time revealed that the patient's foraging spot was covered in C. maculatum. Additionally, field samples of $C$. maculatum wilted in 30 minutes, and the leaves were identical to those in the case images. Many samples also had purple spots that were not easily photographed. Conclusion: This patient likely suffered anoxic brain injury secondary to difficulty with airway management. When confronted with unknown plant toxicity, photographs may fail to capture defining characteristics of plants and wilting may also confound identification.

162. Mercury-Associated Hyponatremia and Endocrine Abnormalities Following a 45-Day Ingestion of an Ayurvedic Medicine

Farah Saleh $^{1}$, Daniel Ovakim ${ }^{1,2}$, Mark Yarema ${ }^{1,3}$, Morgan Riggan ${ }^{1,4}$, Riley Hartmann ${ }^{3,5}$, Scott Lucyk ${ }^{1,3}$, Lily Lum ${ }^{1}$
${ }^{1}$ Poison and Drug Information Service (PADIS), Calgary, AB, Canada. ${ }^{2}$ British Columbia Drug and Poison Information Centre, Vancouver, BC, Canada. ${ }^{3}$ The University of Calgary, Calgary, $A B$, Canada.${ }^{4}$ Western University, London, ON, Canada. ${ }^{5}$ The University of Saskatchewan, Saskatoon, SK, Canada

Background: Ayurvedic medicines may contain lead, mercury, and arsenic. Mercury neurotoxicity is well described in the literature; however, little is known about its potential electrolyte and endocrine abnormalities. We present a case of hyponatremia, hypotestosteronism, hypothyroidism, and elevated urine cortisol after ingestion of a mercury-containing Ayurvedic medicine.

Methods: A 29-year-old male presented to the emergency department with chronic back pain, generalized weakness, numbness, and tingling. The patient admitted to self-treating his back pain with an Ayurvedic supplement for 45 days; his last dose was 8 weeks before presentation. On presentation, the patient's heart rate was 116. Physical exam revealed bilateral bicep and lower extremity fasciculations, abnormal tandem gait along with delusions and hallucinations. Blood testing revealed a sodium of $112 \mathrm{mmol} / \mathrm{L}(133-145)$, TSH $5.8 \mathrm{mU} / \mathrm{L}(0.2-4)$, and testosterone $2.0 \mathrm{nmol} / \mathrm{L}(10.3-29.5)$. Urine testing showed a protein to creatinine ratio of $390.2 \mathrm{mg} / \mathrm{mmol}(<13)$, an AM urine cortisol of $662 \mathrm{nmol} /$ day $(100$ $380)$, a mercury concentration of $708 \mathrm{nmol} / \mathrm{L}(0-50)$, total mercury of $1416 \mathrm{nmol} / \mathrm{v}(0-50)$. Blood and urine tests for arsenic and lead were normal, as was an MRI of the brain and spine. Renal biopsy showed stage 1 membranous nephropathy.

Results: The patient was treated with dimercaprol $5 \mathrm{mg} / \mathrm{kg}$ IM followed by $2.5 \mathrm{mg} / \mathrm{kg}$ IM BID for 8 days, then oral dimercaptosuccinic acid (DMSA) $500 \mathrm{mg}$ TID for 5 days, then $500 \mathrm{mg}$ BID for 2 weeks. The urine mercury concentration 24 hours after completion of DMSA was $194 \mathrm{nmol} / \mathrm{L}$ and total mercury was $331 \mathrm{nmol} / \mathrm{V}$. The patient's neurological symptoms improved and he was subsequently discharged. His cortisol and testosterone concentrations normalized within 6 weeks post-discharge.

Conclusion: Mercury toxicity may result in hyponatremia, endocrine abnormalities, neuropathy, and nephropathy.

\section{Pediatric Birch Oil Ingestion Resulting in Salicylate Toxicity and} Hemodialysis

Kaitlyn Brown ${ }^{1,2}$, Karen Smith ${ }^{3}$, Ron Kirschner ${ }^{3,4}$

${ }^{1}$ Utah Poison Control Center, Salt Lake City, UT, USA. ${ }^{2}$ University of Utah, Salt Lake City, UT, USA. ${ }^{3}$ Nebraska Regional Poison Center, Omaha, NE, USA. ${ }^{4}$ University of Nebraska Medical Center, Omaha, NE, USA

Background: Oil of wintergreen is recognized as a common source of methyl salicylate. Other essential oils containing methyl salicylate include teaberry, spicewood, and sweet birch. Salicylate toxicity following birch oil ingestion has not, to our knowledge, been reported in the medical literature. Hypothesis: Exploratory pediatric ingestion of unfamiliar essential oils may lead to systemic toxicity.

Methods: This is a single patient chart review. A 4-year-old female with developmental delay reportedly ingested 1 ounce of a homemade skin care mixture containing water, coconut oil, and birch oil. She developed rapid breathing and vomiting at home. On arrival at the emergency department, she was initially alert and interactive but soon was described as having altered mental status. Her vital signs were significant for a respiratory rate of 40 breaths/minute. Labs obtained 4.5 hours post ingestion: salicylate concentration $80 \mathrm{mg} / \mathrm{dL}$, venous blood gas $\mathrm{pH} 7.33, \mathrm{pCO}_{2} 29 \mathrm{mmHg}, \mathrm{HCO}_{3}$ $15 \mathrm{mmol} / \mathrm{L}$, lactate $2.6 \mathrm{mmol} / \mathrm{L}$, potassium $4.1 \mathrm{mEq} / \mathrm{L}$, serum creatinine $0.5 \mathrm{mg} / \mathrm{dL}$. She was treated with sodium bicarbonate infusion, potassium replacement, and ondansetron and was transferred to a pediatric hospital for further management. At 10 hours post ingestion, she was still vomiting, had Kussmaul breathing, and remained alert. Labs 10.5 hours post ingestion: salicylate $78.8 \mathrm{mg} / \mathrm{dL}$, arterial $\mathrm{pH} 7.53, \mathrm{pCO}_{2} 20 \mathrm{mmHg}, \mathrm{HCO}_{3}$ $16.8 \mathrm{mmol} / \mathrm{L}$, lactate $1.8 \mathrm{mmol} / \mathrm{L}$, potassium $3.3 \mathrm{mEq} / \mathrm{L}$. 
Results: Dialysis was recommended by the toxicology team. Following a 3-hour hemodialysis run, serum salicylate was $17.5 \mathrm{mg} / \mathrm{dL}$ and bicarbonate infusion was discontinued. Creatinine peaked at $1.36 \mathrm{mg} / \mathrm{dL} 36 \mathrm{~h}$ post exposure. The patient was discharged on hospital day 3 .

Conclusion: This case demonstrates salicylate toxicity from pediatric exploratory ingestion of birch oil. It is critical for clinicians to recognize the potential dangers of methyl salicylate-containing essential oils.

\section{A Case Series of Cassia occidentalis Toxicity in Yemen}

Shaikhah K Al-Otaibi ${ }^{1,2}$, Jameela A Kahtan ${ }^{3}$, Razan N Faisal ${ }^{4}$, Kholoud I Al-Arassi ${ }^{3}$, Ziad N Kazzi ${ }^{1,5}$

${ }^{1}$ Emory University, Atlanta, GA, USA. ${ }^{2}$ King Fahad Hospital of the University/Imam Abdulrahman Bin Faisal University, Dammam, Eastern Province, Saudi Arabia. ${ }^{3} A L$ Thawra Modern General Hospital, Sann'a, Yemen. ${ }^{4}$ King Fahad Specialist Hospital, Dammam, Eastern Province, Saudi Arabia. ${ }^{5}$ Georgia Poison Center, Atlanta, GA, USA

Background: Cassia occidentalis is a plant that belongs to the Calsalpiniaceae family. It grows in South Asia and is used as a homeopathic remedy. The pods contain anthraquinones that deplete glutathione. The toxicity mainly affects the hepatic and central nervous systems. The clinical manifestations include low proteins and significant elevation in transaminases. There are a few reported fatalities in children with no proposed treatment besides supportive care.

Methods: We are reporting four children in Yemen who presented to a hospital after they accidentally ingested an unknown amount of Cassia occidentalis seeds.

Results: The first two children were 4 and 2 years old. They presented on the day of ingestion with vomiting and lethargy. Their condition rapidly deteriorated and they developed irritability, seizures, and coma. Their liver enzymes (AST, ALT) reached $2000 \mathrm{U} / \mathrm{L}$. On the 2 nd day, they died after sustaining cardiac arrest. The third child was a 4-year-old girl who presented 5 days after the ingestion with vomiting and lethargy. Her vital signs were normal and liver enzymes were elevated. On the 9th day, she was started on $N$-acetylcysteine for a 21-hour protocol, and liver enzymes decreased during the treatment period. After stopping $N$-acetylcysteine, AST and ALT increased again. $N$-acetylcysteine was restarted, and the patient's AST and ALT decrease and normalized on the 25th day of admission. The fourth child is a 6-year-old boy who presented on the 5 th day post-ingestion with vomiting. Vital signs and physical exam were normal. AST and ALT were elevated but decreased throughout his admission with supportive treatment.

Conclusion: As the proposed mechanism of Cassia occidentalis liver toxicity is depletion in glutathione, $\mathrm{N}$-acetylcysteine may be used as a therapeutic adjunct to supportive care. Experimental studies are necessary to examine the mechanisms of toxicity and the potential efficacy of $N$ acetylcysteine.

\section{Goldenseal Ingestion Presents with Strychnine-Like Movements}

John A Thompson ${ }^{1,2}$, Nathanael J Mckeown ${ }^{2}$, Robert G Hendrickson ${ }^{1,2}$ ${ }^{1}$ Oregon Health \& Science University, Portland, OR, USA. ${ }^{2}$ Oregon Poison Center, Portland, OR, USA

Background: Goldenseal (Hydrastis canadensis) is a plant with reported anti-inflammatory properties. It contains the isoquinoline alkaloids, berberine, and hydrastine. Berberine has cardiac depressant effects, while hydrastine is reported to have strychnine-like effects.

Methods: This is a single case report. A 19-year-old male went home after overnight observation in an emergency department for ethanol intoxication. His parents held an intervention and instructed him to drink activated charcoal. Later, he was instructed to drink a goldenseal tea. A few hours after drinking the tea, the patient began having small contractions which he attributed to his recent drinking. The contractions steadily increased in severity and frequency. The muscle contractions continued to worsen, causing him to arch his back off his bed. He then presented to the emergency department. Vital signs were notable for HR 140, RR 44, B/P $173 / 81$, temperature 37.3 , and oxygen saturation $91 \%$ on room air. His physical exam was notable for diaphoresis, spasms of large muscle groups, and opisthotonus. His tetanus vaccination was verified to be up to date and he had no report of recent injuries or suspicious wounds on his skin exam or in recent history. His initial laboratory tests were notable for: WBC of 25,000 , lactate of $10.6 \mathrm{mmol} / \mathrm{L}, \mathrm{CK} 8800 \mathrm{U} / \mathrm{L}$, and an anion gap of 25 . The patient was provided fluids and benzodiazepines. His symptoms improved overnight with supportive care.

Results: Hydrastine, an alkaloid present in goldenseal, is reported to cause strychnine-like movements. Historically, it was used to control postpartum hemorrhage as a uterotonic. In animal studies, hydrastine caused convulsions when administered to mice and has similar effects of IPSP of smooth muscle cells as strychnine in guinea pig model.

Conclusion: This case illustrates strychnine-like muscle contractions associated with an ingestion of goldenseal tea.

\section{Tung Tree Nut Ingestion in Three Adult Prisoners}

Matthew Eisenstat, Colin Therriault, Michael Yeh, Jared Cavanaugh, Brent Morgan

Emory University, Atlanta, GA, USA

Background: Aleurites fordii is commonly known as tung nut, tung oil tree, or Chinawood oil tree. It is found in the southeastern USA, China, Taiwan, Japan, and India. The nut is known to be toxic to humans and animals through an unknown mechanism. Outbreaks of illness can occur frequently with children mis-identifying the nuts as edible. We are reporting a case of three prisoners who ingested tung tree nuts while on a work patrol.

Methods: This is a case report involving three patients that was reported to the regional poison center. The poison center was contacted following an ingestion of tung tree nuts by three prisoners. The prisoners immediately began to complain of abdominal pain, nausea, vomiting, dizziness, and throat irritation. A worker on site positively identified the tree that the nuts came from the tung tree. The prisoners were directed to the nearest emergency department for supportive care.

Results: The three patients were taken to a local emergency department where they continued to experience nausea and vomiting predominantly. All three patients had basic lab work performed including complete blood count and complete metabolic panel, all of which was unremarkable. After each patient received a liter of IV fluid and $4 \mathrm{mg}$ of ondansetron, their symptoms resolved in less than 8 hours and they were able to be discharged. Conclusion: Tung nut, while rarely deadly, is a common plant in the southeastern USA that can cause significant symptoms, especially in young patients. Symptoms in previous case series have frequently been described a predominantly nausea, vomiting, and diarrhea. Rehydration and anti-emetics are often enough to support patients through this shortlived toxicity. Poison centers should be able to recognize the tung tree nut as a possible gastrointestinal irritant and direct care appropriately.

\section{A Case of Kratom Poisoning Causing Cardiotoxicity}

Kevin T Baumgartner, Michael E Mullins

Washington University School of Medicine, Saint Louis, MO, USA

Background: Kratom, a substance derived from the leaves of Mitragyna speciosa, is an emerging drug of abuse that is sometimes used for the selftreatment of opioid dependence. Mitragynine, the primary alkaloid present in kratom, has opioid-like and stimulant-like effects, but little is known about its acute or chronic toxicity in humans.

Hypothesis: Kratom may have cardiotoxic effects.

Methods: This is a case report. A 17-year-old male with a history of opioid dependence had been self-treating with kratom for over a year. 
He then ingested a novel high-potency kratom product ("Super K"). He developed nausea, vomiting, palpitations, and anxiety, and then lost consciousness. He regained consciousness after field administration of naloxone. On hospital presentation, his electrocardiogram (EKG) demonstrated markedly prolonged QT interval and bizarre upsloping ST segments. He was also found to be hypokalemic.

Results: The patient's symptoms improved with supportive care and electrolyte repletion, and his EKG normalized. He did not require any additional naloxone. He was ultimately discharged home in good condition. Urine drug testing by liquid chromatography tandem mass spectrometry (LC-MS/MS) confirmed the presence of mitragynine; no other substances were detected on our hospital's extended LC-MS/MS drug screen, and specific serum testing for caffeine was negative. In vitro data suggest that mitragynine may inhibit the rapid delayed rectifier potassium current $\left(\mathrm{I}_{\mathrm{Kr}}\right)$ or might antagonize adenosine A2 receptors. Either interaction could be a potential explanation of the patient's EKG abnormalities and electrolyte disturbance.

Conclusion: Mitragynine, the primary alkaloid present in kratom, may cause tachycardia, QT prolongation, repolarization abnormalities, and hypokalemia. This may be related to inhibition of the cardiac $\mathrm{I}_{\mathrm{Kr}}$ or to adenosine antagonism.

\section{Abstract Withdrawn}

169. Swipe Right: a Comparison of Accuracy of Plant Identification Apps for Identification of Toxic Plants

Jenna Otter, Christian A Tomaszewski

University of California, San Diego, San Diego, CA, USA

Background: Plant exposures remain in the top 25 reported exposures to US Poison Centers. Plant identification applications (ID apps) on smart phones are proposed for identification of toxic plants implicated in exposures; however, none have been validated for this.

Research Question: Can a plant identification app reliably identify toxic plants, and which is most accurate?

Methods: We used three popular iPhone plant ID apps, PictureThis (C) (PT), PlantSnap $\odot(P S)$, and PlantNet@ $(\mathrm{PN})$, to identify commonly encountered toxic plants in public spaces. Apps were used to photograph the entire plant, leaves, and flowers of $\geq 10$ individual plants for each species. Two toxicologists performed plant identification and inter-researcher reliability was confirmed. For each plant species, composite proportion $[95 \% \mathrm{CI}]$ of correct identification was compared between apps.

Results: PT was better at identifying yew (PT 100\% [72-100] vs PS $40 \%$ [17-69] and PN 20\% [6-51]). The best performing app was better than the worst for yellow oleander (PT 93\% [76-99] vs PS 53\% [36-70]) and poison oak (PT 100\% [72-100] vs PS 30\% [11-60]). PT and PN were better than PS for the following: common oleander (PT 98\% [90-100] and PN 100\% [90-100] vs PS 65\% [51-76]); Brugmansia (PT 91\% [7697] and PN 88\% [71-96] vs PS 46\% [32-61]); Datura (PT 100\% [88$100]$ and PN $100 \%$ [84-100] vs PS $43 \%$ [27-61]); tree tobacco (PT $100 \%$ [79-98] and PN 95\% [76-99] vs PS 50\% [33-67]); American pokeweed (PT 100\% [89-100] and PN 100\% [84-100] vs PS 60\% [39-78]); Lantana (PT 87\% [74-94] and PN 87\% [70-95] vs PS 42\% [29-57]). All apps did equally well in identifying pencil cactus, poison ivy, azalea, castor bean, and Chinese evergreen.

Conclusion: PictureThis and PlantNet most often correctly identified toxic plants among the plants photographed. A plant identification app may be a useful tool to assist in identifying toxic plants.

170. Incidence of Acute Hypersensitivity Reactions in Pediatric Patients Receiving Crotalidae Polyvalent Immune Fab

Bryan Corbett ${ }^{1}$, Jenna Otter ${ }^{1}$, Clifford Masom ${ }^{1,2}$, Richard Clark ${ }^{1}$

${ }^{1}$ University of California, San Diego, San Diego, CA, USA. ${ }^{2}$ North Shore

Hospital, Manhasset, NY, USA
Background: Interpretations of studies of acute hypersensitivity reactions in pediatric populations receiving Crotalidae polyvalent immune fab (CPIF) are complicated by small size, wide age ranges, and nonspecific definitions of such reactions.

Research Question: What is the incidence of acute hypersensitivity reactions to CPIF in a pediatric population?

Methods: This is a retrospective chart review of patients aged 13 years or younger who were treated at a children's hospital for crotalid envenomation and received CPIF from November 2006 to 2016. The primary outcome was the presence of an acute hypersensitivity reaction to CPIF and was defined as the development of any of the following symptoms at any time after receiving CPIF: urticaria, wheezing or respiratory distress, angioedema, hypotension, nausea, and/or vomiting. Demographics, CPIF dose to control and total dose, bite location, level of care, and length of stay were also recorded.

Results: Thirty-four patients were treated with CPIF. Ages ranged from 10 months to 13 years. Twenty-one patients $(60 \%)$ were male, $24(70.6 \%)$ were admitted to the ICU, and the median length of stay was 2 days with a range of 1-11 days. One patient, a 4-year-old male, possibly developed an acute hypersensitivity reaction based on our a priori definition. On hospital day (HD) 3, he developed nausea after morphine administration. On HD 4, he vomited after fresh frozen plasma (FFP) administration, and on HD 5, he developed respiratory distress after administration of $20 \mathrm{~mL} / \mathrm{kg}$ each of FFP and packed red blood cells. The closest temporal association of any manifestation to CPIF administration was 5 hours and 49 minutes. Conclusion: Acute hypersensitivity reaction to CPIF occurred in 1/34 $(2.9 \%)$ patients and was likely not related. Such reactions are rare with the use of CPIF in pediatric patients.

171. A Case Series of Maternal Kratom Use and Newborns with Neonatal Abstinence Symptoms

Jennifer S Love ${ }^{1}$, Michael J Moss ${ }^{2}$, John A Thompson ${ }^{1}$, Lauren Murphy ${ }^{1}$, Robert G Hendrickson ${ }^{1}$

${ }^{1}$ Oregon Health \& Science University, Portland, OR, USA. ${ }^{2}$ University of Utah, Salt Lake City, UT, USA

Background: Kratom is a federally unregulated herbal product increasingly used for its opioid and stimulant-like effects, including opioid withdrawal prevention. Few case reports have described neonatal abstinence syndrome (NAS) in infants born to mothers using kratom in pregnancy.

Hypothesis: Infants born to mothers using kratom in pregnancy may have symptoms consistent with opioid withdrawal.

Methods: This is a case series of two patients using Poison Center and medical record data. A modified Finnegan scoring system was used for withdrawal assessment. Patient A: A full term, small for gestational age female was born by spontaneous vaginal delivery (SVD). On day of life (DOL) 1, she developed jitteriness, inconsolability, and increased stooling. Mom denied drug use but reported drinking kratom tea during pregnancy. Patient B: A full term female was born by SVD. On DOL 1, she developed increased fussiness, jitteriness, sneezing, increased respiratory rate, and poor feeding. Mom had a negative urine drug screen on admission but reported taking kratom during pregnancy.

Results: At birth, patient A's calcium and glucose were 7.6 and $41 \mathrm{mg} / \mathrm{dL}$, respectively. She was started on D10W with formula supplementation and continued breastfeeding. Her glucose and calcium improved over several days. Her maximum NAS score was 11. She did not require morphine and was discharged home on DOL 5. At birth, patient B's calcium and glucose were 8.1 and $56 \mathrm{mg} / \mathrm{dL}$, respectively. She was breast and bottle-fed. Her glucose improved in 48 hours. Her maximum NAS score was 11. She did not require morphine and was discharged home on DOL 4.

Conclusion: Infants born to mothers using kratom may show symptoms similar to NAS. Additionally, infants may be hypoglycemic at birth. Future research should examine kratom transmission in utero and during breastfeeding and the effects of kratom on infants. 
172. Adolescent Suicidal Ingestions: Agents Reported to New Jersey Poison Information and Education System from 2000 to 2017

Jeena E Jacob ${ }^{1,2}$, Brian Kaiser ${ }^{1}$, Bruce Ruck ${ }^{2}$, Christine Ramdin ${ }^{1}$, Diane Calello ${ }^{1,2}$

${ }^{1}$ Rutgers New Jersey Medical School, Newark, NJ, USA. ${ }^{2}$ New Jersey Poison Information and Education System, Newark, NJ, USA

Background: Suicide is the second leading cause of death in adolescents in the USA. Our previous research shows an increase in self-poisoning in adolescents ages 10-14 and 15-19 reported to the New Jersey Poison Information and Education System (NJPIES) from 2000 to 2017.

Research Question: What are the most common agents used in overdose with suicidal intent in the adolescent population reported to NJPIES from 2000 to 2017 and is there a change in the incidence of these agents during this time period?

Methods: This was a retrospective study of cases of self-poisoning with suicidal intent reported to NJPIES from 2000 to 2017. Data was collected on patients ages 10-19 years old with reason for ingestion coded as intentional suspected suicide.

Results: A total of 18,050 cases were identified. There were 2.7 vs 2.8 ingestions/case in the 10-14 and 15-19 age groups, respectively ( $p=$ 0.029 ). The most common drug classes/agents ingested overall were NSAIDS $(24.6 \%)$. This was followed by acetaminophen $(21.5 \%)$, SSRIs $(12.1 \%)$, antipsychotics $(6.6 \%)$, and benzodiazepines $(6.4 \%)$. The 10-14 age group had increases over the study period in ingestion of SSRIs (3.7 vs $5.5 \%, p=0.019)$, antipsychotics ( 1.3 vs $2.2 \%, p=$ $0.011)$, and diphenhydramine ( 0.87 vs $1.9 \%, p=0.002)$. The $15-19$ age group had an increase in benzodiazepines ( 1.7 vs $2.6 \%, p=0.003)$, SSRIs (3.7 vs $5.6 \%, p=0.003)$, and diphenhydramine $(0.43$ vs $1.7 \%$, $p<0.0001)$. The $15-19$ age group had a decrease between 2000 and 2017 in acetaminophen ingestions $(8.7$ vs $1.7 \%, p=0.001)$ and aspirin ingestions ( 3.1 vs $1.3 \%, p<0.0001)$.

Conclusion: Ingestions with benzodiazepines, SSRIs, antipsychotics, and diphenhydramine increased in adolescents during 2000-2017. Ingestions involving acetaminophen and aspirin decreased. This information can help focus provider and public health efforts in suicide prevention in this at-risk population.

173. Prolonged Obtundation and Choreoathetosis Following Pediatric Exploratory Phencyclidine (PCP) Ingestion with Serum Concentration Measurement

Julia Rood ${ }^{1}$, Brian F White ${ }^{2}$, Alyssa R Thomas ${ }^{1}$, Jeanine Ronan ${ }^{3}$, Julie Fitzgerald $^{4}$, Kevin C Osterhoudt ${ }^{5,6}$

${ }^{1}$ Pediatric Residency Program, Children's Hospital of Philadelphia, Philadelphia, PA, USA. ${ }^{2}$ Division of Neurology, Children's Hospital of Philadelphia, Philadelphia, PA, USA. ${ }^{3}$ Division of General Pediatrics, Children's Hospital of Philadelphia, Philadelphia, PA, USA. ${ }^{4}$ Division of Critical Care Medicine, Children's Hospital of Philadelphia, Philadelphia, PA, USA. ${ }^{5}$ Section of Clinical Toxicology, Children's Hospital of Philadelphia, Philadelphia, PA, USA. ${ }^{6}$ Center for Injury Research and Prevention, Children's Hospital of Philadelphia, Philadelphia, PA, USA

Background: Phencyclidine is an NMDA receptor antagonist, nicotinic antagonist, dopamine ${ }_{2}$ agonist, and a classically recognized drug of abuse; modern reports of intoxication of young children are rare.

Research Question: What are the clinical findings and symptom duration of PCP poisoning in a child with exploratory exposure?

Methods: This is an observational description of a single case.

Results: An exploratory 23-month-old, $14 \mathrm{~kg}$, boy became unresponsive 20 minutes after drinking a liquid encountered at home. Respirations were $10 /$ minute, pupil diameter $2 \mathrm{~mm}$, blood glucose $93 \mathrm{mg} / \mathrm{dL}$, there was no response to naloxone $2 \mathrm{mg} \mathrm{IV}$, and he was endotracheally intubated for 1 day. A urine drug of abuse immunoassay (Vitro® 4600 Chemistry
System) was positive only for PCP; blood ethanol was negative. On day 3 , the boy remained obtunded, with intermittent agitation, and developed choreoathetosis and dystonia which were treated with diphenhydramine, diazepam, and risperidone. Brain MRI was normal. On day 5, withdrawal to pain and purposeful eye opening returned, but his face was expressionless without nystagmus; choreiform movements subsided day 8 and he could walk on day 10. Advanced toxicological testing including gas + liquid chromatography / tandem mass spectrometry, with targeted testing for synthetic cannabinoids, did not identify any other drugs of concern. Serum PCP quantitation was performed: $255 \mathrm{ng} / \mathrm{mL}$ at 6 hours and $32 \mathrm{ng} / \mathrm{mL}$ at 196 hours. Evaluation 2 months after ingestion noted return to neurodevelopmental baseline.

Conclusion: PCP intoxication was manifest by unresponsiveness, intermittent agitation, a distinctively expressionless face, choreoathetosis, and dystonia; symptom duration approached 10 days. These findings may seem surprising, but are consistent with case series from the 1970s which reported choreoathetoid movement disorder $>50 \%$ of child cases and symptoms persisting up to 200 hours. A serum PCP concentration of $255 \mathrm{ng} / \mathrm{mL}$ is high compared to published pediatric experience. PCP dissolved in liquid may pose particular risk to children.

174. A Retrospective Review of Unintentional "Snake" Firework Exposures Reported to a Poison Center

Adrienne R Hughes ${ }^{1,2}$, Robert G Hendrickson ${ }^{1,2}$

${ }^{1}$ Oregon Health and Science University, Portland, OR, USA. ${ }^{2}$ Oregon Poison Center, Portland, OR, USA

Background: "Snake fireworks," also called "black snakes" or "glow worms," are small tablets that burn to create long black snaking tubes of ash. Snake fireworks contain carbon and small amounts of sulfur, arsenic, or barium. Although considered nontoxic by manufacturers, severe barium toxicity has occurred with intentional overdose. Little information currently exists regarding unintentional ingestion of snake fireworks.

Methods: This is a retrospective study of snake firework exposures reported to a single US poison control center between July 2009 and August 2019. Cases were identified using the Oregon Poison Center (OPC) electronic medical record (Toxicall®). Calls regarding snake fireworks using "barium," "black snakes," "snake fireworks," and "glow worms" were reviewed to determine incidence of calls, demographic details, clinical effects, management sites, and medical outcomes.

Results: Of the 45 cases, $64 \%$ were male, all were $<8$ years old (range $1-$ 8 years; mean 3.04 years; median 3 years). Most cases resulted in no clinical effects $(80 \%)$. The most common reported clinical effects were diarrhea $(6.6 \%)$, hypokalemia $(6.6 \%)$, vomiting $(4.4 \%)$, and abdominal pain $(4.4 \%)$. Twenty-six cases were followed to a known medical outcome: 0 -major, 2-moderate, 4 -minor, and 20-no effect. Thirty-six cases were managed at home, 9 were referred to an ED, 7 discharged, and 2 admitted to the hospital ward. Mean serum potassium concentration in 8 cases was $3.7 \mathrm{mEq} / \mathrm{L}$ (range $2.1-4.7 \mathrm{mEq} / \mathrm{L}$ ). In six cases (aged 48 years), the firework was ingested after it was mistaken for candy; two mistook it for a Tootsie Roll@ and one mistook it for black licorice.

Conclusion: The majority of unintentional "snake" firework exposures occur in children and result in no toxicity. The most common adverse effects are gastrointestinal and mild hypokalemia. All symptomatic patients recovered with routine supportive care. Based on this data, it appears that the majority of unintentional "snake" firework exposures can be managed at home and do not require referral to a healthcare facility.

175. Unintentional Ingestion of Pimobendan (Vetmedin): 8 Years of Experience at a Single Poison Control Center

Danielle M Fitzpatrick, Austin M Schatzman, Andrew J Tominaga, Jane E Miloradovich

The Poison Control Center at The Children's Hospital of Philadelphia, Philadelphia, PA, USA 
Background: Each year, the Poison Control Center (PCC) receives numerous calls about people ingesting medications meant for pets. Pimobendan (brand name Vetmedin ${ }^{\circledR}$ ) is a calcium sensitizer and selective phosphodiesterase-3 inhibitor with positive inotropic and vasodilator effects used in canine/feline heart failure. To date, there has been minimal research exploring the effects of this medication in humans. Theoretical adverse effects based on small studies in animals include headache, dizziness, hypotension, and tachycardia.

Research Question: We describe a US Poison Control Center's longitudinal experience with pimobendan human exposure cases.

Methods: This is a retrospective, observational review of de-identified data from a single poison control center from March 2011 to October 2019. Human exposure cases coded as ingestions of either pimobendan or Vetmedin were included.

Results: Of 79 cases analyzed, $80 \%$ involved females. Median age was 57 years, with a range of 11 months to 93 years. Symptoms occurred in 11 patients and were categorized as mild $(n=5,45 \%)$ or moderate $(n=6,55 \%)$. Reported symptoms included headache, dizziness, tachycardia, hypotension, dysrhythmia, fatigue, diuresis, flushing, worsening baseline tinnitus, and difficulty standing. However, most symptomatic patients had also taken antihypertensives, diuretics, sedatives, or a combination thereof. Eighteen patients $(23 \%)$ were either en route to a healthcare facility $(\mathrm{HCF})$ or referred to a $\mathrm{HCF}$ by the PCC, and none of these patients were admitted to a HCF.

Conclusion: Our data represents a significant number of exposures to pimobendan in humans. Based on the examination of the cases called into the Philadelphia PCC, pimobendan appears relatively safe for ingestion in humans. Given our case review, it is reasonable to advise callers that they may experience headache, dizziness, tachycardia, hypotension, and/or flushing, especially when additional medications with synergistic effects are taken concomitantly.

176. A Needs Assessment for a Standardized Toxicology Curriculum in Emergency Medicine Residency

Brian Bush ${ }^{1}$, James $\mathrm{Ahn}^{1}$, Navneet Cheema ${ }^{1}$, The Emergency Medicine Education Research Alliance (EMERA) ${ }^{2}$

${ }^{1}$ Section of Emergency Medicine, University of Chicago, Chicago, IL, USA. ${ }^{2}$ Chicago, IL, USA

Background: Expertise in toxicology is geographically concentrated and unequally distributed across training programs. Currently, no literature investigates the need for the development of a standardized toxicology curriculum for emergency medicine (EM) trainees.

Hypothesis: We hypothesize that EM learners are uncomfortable with core toxicology content and would be interested in a standardized toxicology curriculum.

Methods: This is a needs assessment surveying EM residents' comfort with core content in toxicology and faculty understanding of their toxicology curriculum. Residents at nine EM programs were provided a survey designed through REDCap (8.9.2, Vanderbilt University, 2019). Current toxicology curriculum, interest in a standardized toxicology curriculum, comfort with core toxicology concepts for board examinations, and in clinical practice were queried. A faculty representative from each participating residency program completed a separate survey. The existence of a toxicology curriculum, total didactic time given to toxicology, curriculum delivery methods, and presence of a rotation in toxicology for EM residents were queried.

Results: Preliminarily, 123/433 resident and 8/9 faculty answered the survey; $29.3 \%$ of the respondents felt comfortable with their toxicology exposure during residency and $37.4 \%$ felt comfortable with their toxicology knowledge in anticipation of board examination; $65.9 \%$ of the respondents acknowledged having a toxicology curriculum. From the $34.1 \%$ without, $90.3 \%$ responded that they would be interested in a formal toxicology curriculum. Six out of nine programs have a toxicology curriculum. Seven out of nine programs require a resident toxicology rotation. Six out of nine programs allocated $5 \%$ of total didactic time to toxicology. Simulation and lectures were the most common teaching methodology.

Conclusion: While emergency medicine residencies often have toxicology content, preliminary results showed that EM residents are still uncomfortable with the core toxicology content and would be interested in a standardized toxicology curriculum. This ongoing needs assessment demonstrates the need for the development of such a curriculum to assist EM learners in building knowledge and comfort with toxicology content.

177. Time to Medication Administration: Comparison of Medications from the ED Automated Dispensing Machines Vs. Medications from Main Hospital Pharmacy

\section{Kathryn T Kopec ${ }^{1}$, Alyshia Wiggins ${ }^{2}$, Anna R Dulaney ${ }^{3}$}

${ }^{1}$ Carolinas Medical Center, Charlotte, NC, USA. ${ }^{2}$ Virginia Commonwealth University Health System, Richmond, VA, USA. ${ }^{3}$ North Carolina Poison Control Center, Charlotte, NC, USA

Background: Medication administration time is an operational measurement emergency department (ED) measure when evaluating throughput times. Patient morbidity and mortality are decreased when medications are given quickly. In our ED, medications are either obtained from automated dispensers or admixed in the main pharmacy. Hospital policy requires ED orders be considered "stat" therefore must be started within 30 minutes of order placement.

Hypothesis: We hypothesized time to administration of medications from the pharmacy is significantly longer than medications from ED automated dispensers.

Methods: Two hundred ED patients were included between February 1 and September 1, 2018. One hundred patients received the following IV admixtures dispensed from the pharmacy: $\mathrm{N}$-acetylcysteine $(n=6)$, CroFab ${ }^{\circ}(n=2)$, Kcentra ${ }^{\circledR}(n=14)$, fluids with potassium $(n=27)$, sodium bicarbonate $(n=4)$, or insulin $(n=47)$. The other 100 patients received the following IV medications, from ED automated dispensers: alteplase $(n=20)$, clindamycin $(n=20)$, vancomycin $(n=20)$, cefazolin $(n=20)$, or levetiracetam $(n=20)$. We examined time from order placement to time of medication administration to the patient.

Results: Time from order placement to administration of medications dispensed from pharmacy was as follows: $6 \%$ within 30 minutes, $36 \%$ within 31-60 minutes, and 58\% over 61 minutes. Thusly, $94 \%$ of medications from pharmacy did not meet stat order standards. Time from order placement to administration of medications dispensed from ED automated dispensers was as follows: $13 \%$ before order placement, $52 \%$ within 30 minutes, $17 \%$ within $31-60$ minutes, and $18 \%$ over 61 minutes. Accordingly, $65 \%$ of medications dispensed from the ED automated dispensers met the stat order standard. A Mann-Whitney $U$ test showed a significantly shorter time to administration in the automated dispenser group compared to the medication from pharmacy group $(p<0.05)$. Conclusion: Medications obtained from pharmacy have a significantly longer time to administration than those dispensed from ED automated machines. This needs further investigation to determine effects on patient morbidity and mortality.

Publisher's Note Springer Nature remains neutral with regard to jurisdictional claims in published maps and institutional affiliations. 